\title{
Effect of Boundary Conditions on Propagation and Morphology of Premixed Flames in Narrow Conduits
}

\author{
Orlando J. Ugarte-Almeyda
}

Follow this and additional works at: https://researchrepository.wvu.edu/etd

\section{Recommended Citation}

Ugarte-Almeyda, Orlando J., "Effect of Boundary Conditions on Propagation and Morphology of Premixed Flames in Narrow Conduits" (2015). Graduate Theses, Dissertations, and Problem Reports. 6846.

https://researchrepository.wvu.edu/etd/6846

This Dissertation is protected by copyright and/or related rights. It has been brought to you by the The Research Repository @ WVU with permission from the rights-holder(s). You are free to use this Dissertation in any way that is permitted by the copyright and related rights legislation that applies to your use. For other uses you must obtain permission from the rights-holder(s) directly, unless additional rights are indicated by a Creative Commons license in the record and/ or on the work itself. This Dissertation has been accepted for inclusion in WVU Graduate Theses, Dissertations, and Problem Reports collection by an authorized administrator of The Research Repository @ WVU.

For more information, please contact researchrepository@mail.wvu.edu. 


\title{
Effect of Boundary Conditions on Propagation and Morphology of Premixed Flames in Narrow Conduits
}

\author{
Orlando J. Ugarte-Almeyda
}

\author{
Dissertation submitted \\ to the Statler College of Engineering and Mineral Resources \\ at West Virginia University
}

in partial fulfillment of the requirements for the degree of

Doctor of Philosophy in

Mechanical Engineering

V'yacheslav Akkerman, Ph.D., Chair

Vitaly V. Bychkov, Ph.D.

Donald Ferguson, Ph.D.

Hailin Li, Ph.D.

John M. Kuhlman, Ph.D.

Gregory J. Thompson, Ph.D.

Department of Mechanical and Aerospace Engineering

\author{
Morgantown, West Virginia \\ 2015
}

Keywords: combustion, channels, isothermal, thermal expansion, obstacles. Copyright 2015 Orlando Ugarte 


\section{ABSTRACT}

\section{Effect of Boundary Conditions on Propagation and Morphology of Premixed Flames in Narrow Conduits}

\section{Orlando J. Ugarte-Almeyda}

Boundary conditions play a key role in the evolution and morphology of flame fronts, especially when combustion occurs in narrow chambers. The burning intensity and the flame-generated flow can be significantly modified by the momentum and energy transferred at the walls, which are further modified by the exothermal nature of the process. In this work, the effect of the wall roughness and thermal conditions on the flame propagation is explored. Specifically, conduits with and without obstacles, having adiabatic or isothermal walls, are investigated.

Wall friction constitutes one of the main reasons of spontaneous flame acceleration in narrow pipes. Although this phenomenon has been intensely studied, the researchers have focused on the mechanistic scenario of the combustion intensification, induced by the wall friction, putting less emphasis on the heat exchanged at the walls. In this study, besides the adiabatic condition, the surfaces have been kept at multiple constant temperatures in order to explore the wall thermal effects on the burning process, recognizing its potential to diminish or even quench the reaction.

Moreover, the inclusion of solid obstacles at the pipe walls provides a mechanism of extremely fast flame acceleration, which is driven by an intense jet-flow generated by the delayed combustion occurring between obstacles. In this work, the flame dynamics promoted in the obstructed configuration is analyzed, comparing the attained acceleration rates to other mechanisms such as that generated by the wall friction and the so-called finger flame evolution.

For this purpose, a parametric study provided by extensive fully-compressible numerical simulations of the combustion and hydrodynamic equations is performed. The geometry is primary given by 2D channels, although cylindrical 'smooth' tubes have been also considered. The wall conditions include non-slip walls and slip walls with obstacles; adiabatic and isothermal, with the fuel characterized by the thermal expansion coefficient. Four regimes of flame propagation in isothermal 'smooth' channels have been identified, for flames propagating a distance around 100-150 times the flame thickness: (i) no flame propagation or extinction; (ii) linear flame velocity; (iii) almost-constant flame propagation speed; and (iv) oscillating flame velocity. In the obstructed configuration, the developing of turbulent and laminar combustion regimes at the early stages of the process have been identified in relation to the obstacles size and spacing, including a finger flame-like limit when small enough obstacles are in place. 


\section{Dedicatory}

To Him, who upholds all things by the word of His power.

(Heb. 1:3) 


\section{Acknowledgements}

As I write this section, I realize that this work is somehow the sum of multiple collaborations, with most of them out of the strictly speaking scientific field. People that God used to guide me along this journey, even when I was not aware of.

Starting from the beginning, I should thank Dr. Barbero and Dr. Prucz for their constant support and guidance. My former advisers Dr. Smith and Dr. Lewellen, who gave me the opportunity to work as a researcher and taught me many things about the job. Also my gratitude to my friends and classmates in the early days: Hermann, Omar, Antonio, Aaron and my office mates in ESB G20 and ERB 114. It is great to know that you are doing very well.

But this project would not been possible without the help of my adviser Dr. V'yacheslav Akkerman, who included me in his group in a difficult time. Thanks to him I have been able to work on this incredible and challenging field, Combustion, in which I intend to stay for the years to come. Thanks very much Dr. Akkerman.

My gratitude also to my research group, Berk, Sinan, Serdar, Ram and Jad. Thanks for teaching me while we were all learning. Thanks to Dr. Vitaly Bychkov and Dr. Damir Valiev, our collaborators in Sweden, for sharing with us your experience and knowledge. Thanks so much to our 'big' group CAFEE, it was a pleasure belong to such a great team.

But above all, I should thanks my Lord, who made all things possible, for giving me the most wonderful gift ever, my wife, Patricia. Thanks for taking care of our parents and family, making every reunion an unforgettable time. Thanks Father for giving us the best friends in Christ Church of Morgantown. Thanks for our pastor, Peter Jones and family, for Dr. Moss and family, who taught us many things, most of them without saying a word, thanks for all my brothers and sisters in the faith, who showed us a little piece of heaven every Sunday.

God bless you all. 


\section{Table of Contents}

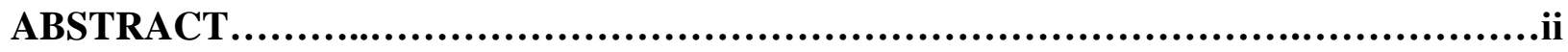

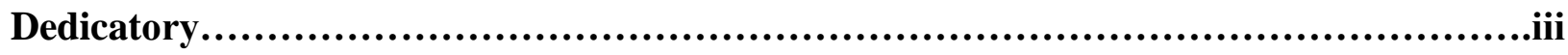

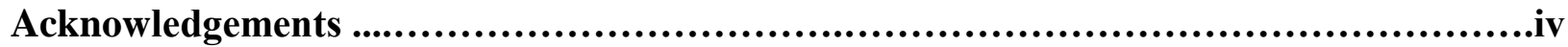

List of Figures.......................................................................................................................................... vii

List of Symbols .......................................................................................................................................... xii

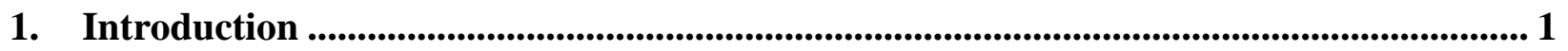

$1.1 \quad$ Flame Dynamics in Premixed Combustion................................................................ 2

1.2 The Importance of Micro Combustion......................................................................... 5

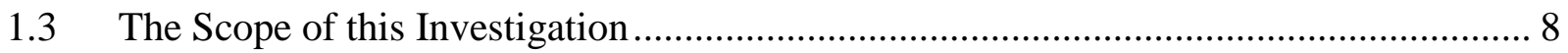

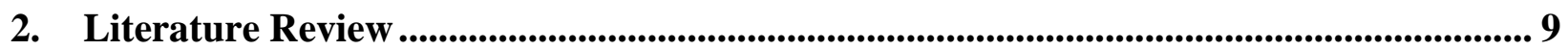

2.1. Effect of Turbulence on the Flame Dynamics .............................................................. 9

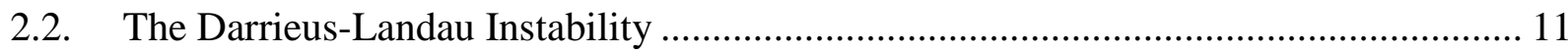

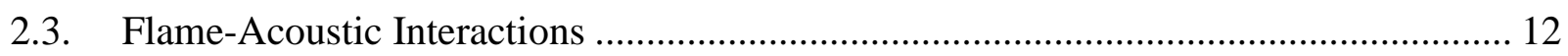

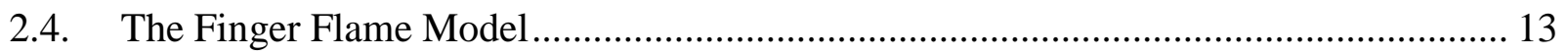

2.5. The Shelkin Mechanism of Flame Acceleration ......................................................... 15

2.5.1. The Laminar Flame Approximation .................................................................. 15

2.5.2. Laminar Flame Acceleration produced by Wall Friction ....................................... 16

2.6. Ultra-Fast Flame Acceleration in Obstructed Conduits .................................................. 18

2.7. Flame Propagation in Non-Adiabatic Tubes and Channels .......................................... 20

2.8. Combustion Characteristics once DDT is triggered.................................................... 21

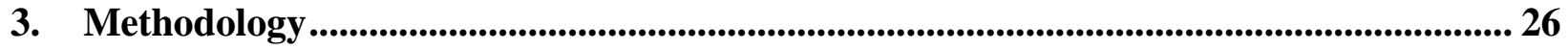

3.1 Description of the Numerical Approach …………..................................................... 26

3.2 Numerical Simulations Set Up .......................................................................... 28

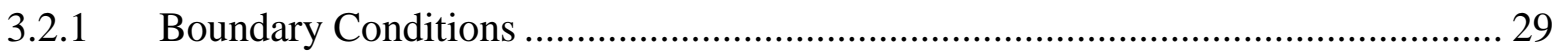

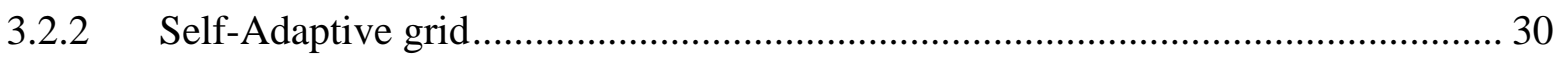

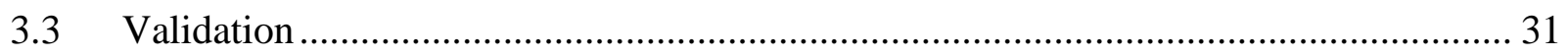

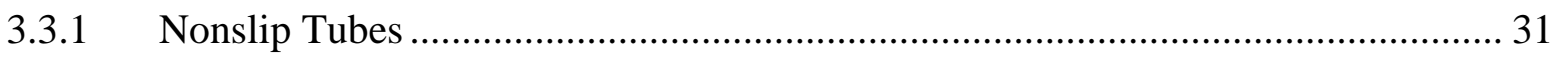




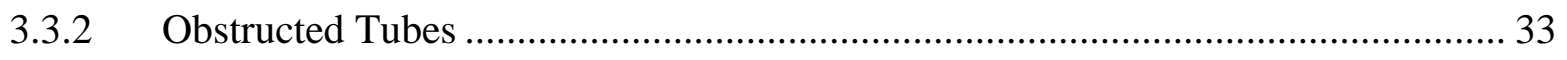

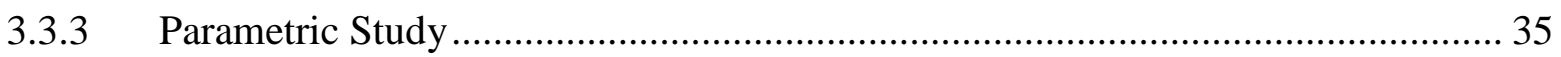

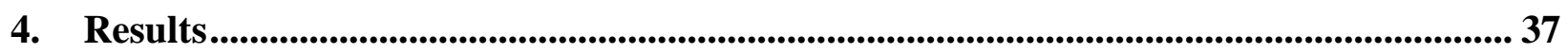

4.1 Flame Dynamics in ‘Smooth' Channels with Isothermal Walls ..................................... 37

4.1.1 Wall Temperature Effect on the Flame Propagation ............................................ 38

4.1.2 Flame Velocity in Isothermal Conditions ................................................................ 44

4.1.3 Very High Wall Temperature Conditions ........................................................... 48

4.1.4 Thermal Expansion Effect on the Flame Propagation Dynamics ........................... 50

4.1.5 Enhancing Flame Propagation at the Walls .......................................................... 54

4.1.6 Channel Width Effect ...................................................................................... 59

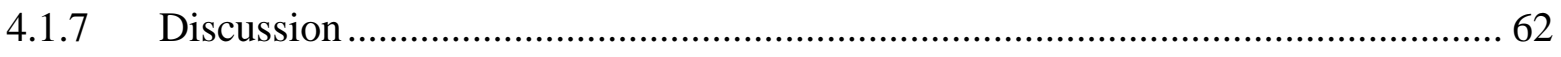

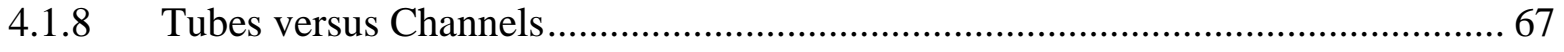

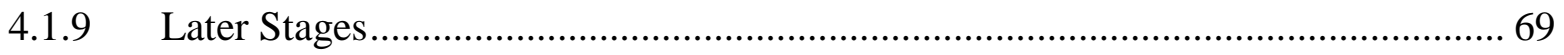

4.2 Flame Dynamics in Obstructed Channels …………...................................................... 71

4.2.1 Obstacles Size Effect on the Flame Propagation .................................................. 72

4.2.2 Effect of the Channel Width ........................................................................... 74

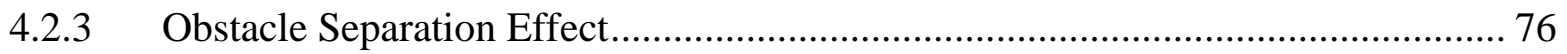

4.2.4 Isothermal Walls Effect …………………................................................. 79

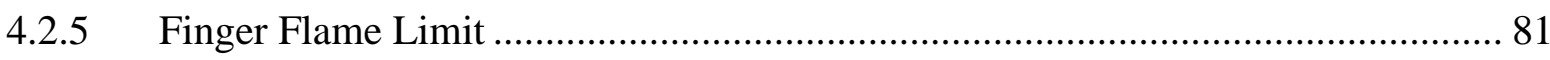

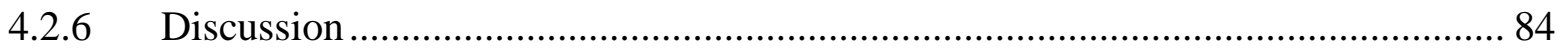

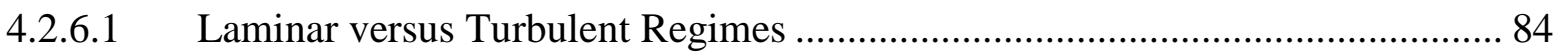

4.2.6.2 Flame Propagation Regimes in Obstructed Channels ....................................... 88

4.2.6.3 Flame Acceleration Comparison: Obstacles vs Wall Friction ............................. 90

5. Conclusions and Future Work ................................................................................... 93

5.1 Conclusions: 'Smooth' Pipes ……………………..................................................... 93

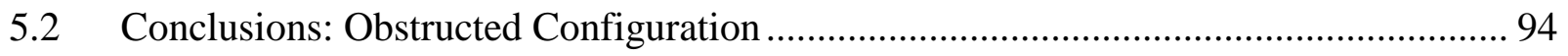

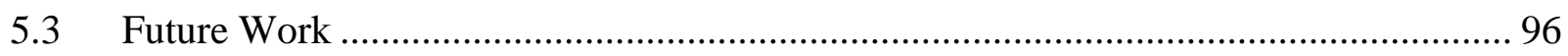

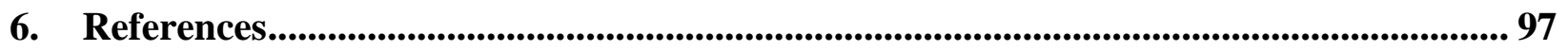




\section{List of Figures}

Figure 1.1. World energy consumption and prediction by fuel type, 1990-2040 [1]................. 1

Figure 1.2. Illustration of the mechanism of premixed flame propagation. ............................... 2

Figure 1.3. a) Scaled temperature of a planar flame propagation. b) Distribution of the scaled density (1), flow velocity (2) and fuel fraction (3) [4] .............................................. 4

Figure 1.4. Gravimetric and volumetric energy densities of diverse materials [8-14]............... 6

Figure 1.5. Micro turbine rotor [15], left. Rotary internal combustion engine [16], right............ 6

Figure 1.6. The power density, calculated by the 'cube-square' law (dotted) and by including convective losses (solid), and efficiency (dashed) of a simplified combustor are shown in relation to its length scale (1) and freely propagating flame thickness $\left(L_{f}\right)$. Results are based on a fuel with specific energy value of $44 \mathrm{MJ} / \mathrm{Kg}$, laminar flame speed $U_{f}=42 \mathrm{~cm} / \mathrm{s}$, and

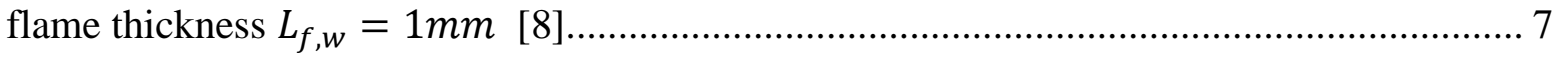

Figure 2.1. The DDT stages as described by Zeldovich et al. [19] .......................................... 9

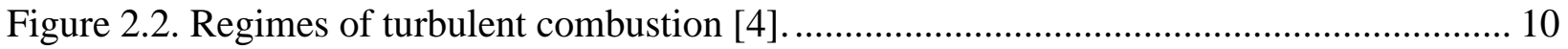

Figure 2.3. Thermal stabilization of the Darrieus-Landau instability. Courtesy of V. Bychkov.. 11

Figure 2.4. 'Finger' flame model of flame propagation. ..................................................... 13

Figure 2.5. Comparison of the time scales of the flame propagation stages observed in finger flames, as predicted analytically (solid) and found experimentally (markers) [30]............ 14

Figure 2.6. a) Velocity profile of the unburned mixture, given by Equation (5) [35]. b) Acceleration rate versus Reynolds number related to the flame propagation. Dashed and solid lines are given analytically by Eq. (7) and Eq. (8). Empty and filled markers represent the calculated acceleration rates from the simulations of channels and axisymmetric tubes, respectively $[35,36]$ 17

Figure 2.7. Flame propagation observed in obstructed channels as described by Valiev et al. [42]. Temperature (top) and Velocity (bottom) distribution, $\Theta=8, \Delta Z / R=0.25, \alpha=2 / 3 \ldots . .19$

Figure 2.8. Schematic of the evolution of a cellular detonation front, as described by the trajectory of the triple points (e.g. A, B, C and D). Cell parameters cell size $\lambda$ and cell length $L_{c}$ are also shown [49]. 22 
Figure 2.9. Characteristic dimensions describing the channel with obstacles investigated in [56], where the charctaeristic size $L^{\prime}$ is presented. 24

Figure 3.1. Grid domain and boundary conditions utilized for unobstructed pipes. .................. 28

Figure 3.2. Grid domain and boundary conditions utilized for obstructed channels.................. 29

Figure 3.3. Schematic of the grid used in the numerical simulations [37] ............................... 30

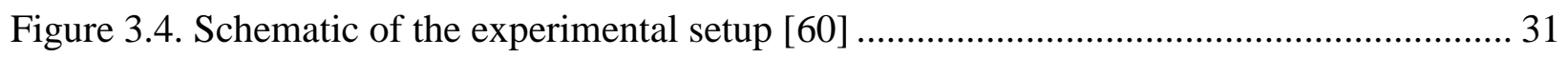

Figure 3.5. Evolution of the scaled flame tip velocity $U_{t} / U_{f}$ versus the scaled time given by $\tau=$ $t U_{f} / R_{t u}$ in a tube of radius $R_{t u}=0.25 \mathrm{~mm}$., calculated numerically and experimentally [59] 32

Figure 3.6. Evolution of the scaled flame tip velocity $U_{t} / U_{f}$ versus the scaled time given by $\tau=$ $t U_{f} / R_{t u}$ in a tube of radius $R_{t u}=0.50 \mathrm{~mm}$., calculated numerically and experimentally [59] 33

Figure 3.7. Optical module of the experimental obstructed channel [61] 34

Figure 3.8. Results obtained experimentally (blue) [61] and numerically (red). Scaled flame velocity versus the scaled flame tip position, $\alpha=1 / 3, \Delta Z / R_{c h}=2$ 34

Figure 3.9. Results obtained experimentally (blue) [61] and numerically (red). Scaled flame

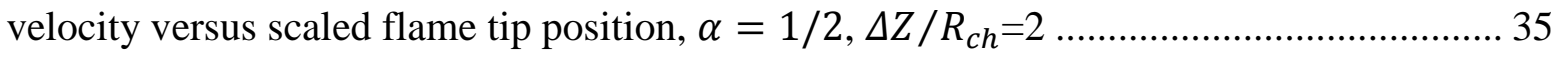

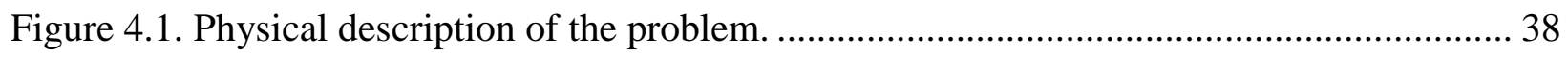

Figure 4.2. Flame propagation in adiabatic (left) and isothermal $T_{w}=300 \mathrm{~K}$ (right) slip channels, $R_{c h}=10 L_{f}$ 39

Figure 4.3. Temperature distribution of the flame propagation in channels (upper half shown), $R_{c h}=10 L_{f}, \Theta=7$. a) adiabatic, b) isothermal, $T_{w}=300 \mathrm{~K}$ and c) isothermal, $T_{w}=800 \mathrm{~K}$. 40

Figure 4.4. Temperature contours of the flame propagation in channels (upper half shown) with $R_{c h}=10 L_{f}, \Theta=7$, and different wall conditions a) adiabatic, b) isothermal $T_{w}=300 \mathrm{~K} .41$

Figure 4.5. Temperature contours in isothermal channels (upper half shown), $T_{w}=800 \mathrm{~K}$, with $\Theta=7, R_{c h}=10 L_{f}$ 42

Figure 4.6. Temperature distribution and streamlines of the flame propagation in channels (upper half shown), $R_{c h}=10 L_{f}, \Theta=7$, a) adiabatic and b) isothermal $T_{w}=300 \mathrm{~K}$ 43

Figure 4.7. Flame velocity evolution at different wall conditions. 44 
Figure 4.8. Linear acceleration regime at different wall temperatures. 45

Figure 4.9. Slopes of the linear acceleration observed in the early stages of flame propagation for the cases presented in Figure 4.8. 46

Figure 4.10. Temperature distribution of the flame propagation in channels (upper half shown) with $\Theta=7, R_{c h}=10 L_{f}$, and different wall temperatures a) $T_{w}=300 K$, b) $T_{w}=800 K . .47$

Figure 4.11. Temperature distribution of the flame propagation in a channel (upper half shown) with walls at $T_{w}=1200 \mathrm{~K}, \Theta=7, R_{c h}=20 L_{f}$. a) $\tau_{f}=0.674$, b) $\tau_{f}=1.24$, c) $\tau_{f}=2.42$

Figure 4.12. Temperature contours of the flame propagation with walls at $T_{w}=1200 \mathrm{~K}, \Theta=7$, $R_{c h}=20 L_{f}$ 50

Figure 4.13. Temperature distribution and streamlines of the flame propagation in a channel (upper half shown) with walls at $\mathrm{T}_{W}=1200 \mathrm{~K}, \Theta=7, \mathrm{R}_{c h}=20 L_{f}$ 50

Figure 4.14. Temperature distribution of the flame propagation in channels (upper half shown) at different times a) $\tau_{f} \sim 0$, b) $\tau_{f} \sim 1.225$, c) $\tau_{f} \sim 2.925 . \Theta=5, \mathrm{R}_{c h}=5 L_{f}, \mathrm{~T}_{W}=300 K . \ldots . .51$

Figure 4.15. Temperature distribution of the flame propagation in channels (upper half shown) at different times a) $\tau_{f} \sim 0$, b) $\tau_{f} \sim 1.1$, c) $\tau_{f} \sim 2.27 . \Theta=7, \mathrm{R}_{c h}=5 L_{f}, \mathrm{~T}_{W}=300 \ldots \ldots \ldots \ldots . .52$

Figure 4.16. Evolution of the flame position along the channel centerline for flame extinction

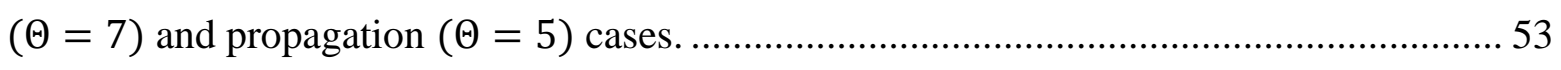

Figure 4.17. Maximum temperature registered in the channel along the process. ..................... 54

Figure 4.18. Temperature distribution of the flame propagation in a channel (upper half shown) with walls at $\mathrm{T}_{W}=800 \mathrm{~K}, \Theta=5, \mathrm{R}_{c h}=10 L_{f}$. a) $\tau_{f} \sim 0.675$, b) $\tau_{f} \sim 1.45$, c) $\tau_{f} \sim 2.475 \ldots 55$

Figure 4.19. Temperature distribution of the flame propagation in a channel with walls at $\mathrm{T}_{W}=$ $800 K, \Theta=9, \mathrm{R}_{c h}=10 L_{f}$. a) $\tau_{f}=3.15$, b) $\tau_{f}=9.99$, c) $\tau_{f}=19.6 \ldots \ldots \ldots \ldots \ldots \ldots \ldots \ldots \ldots \ldots . . .56$

Figure 4.20. Flame position as reported at two locations, centerline and near the wall............. 57

Figure 4.21. Flame velocity corresponding to the cases shown in Figure 4.20 ....................... 57

Figure 4.22. Flame velocity at the channel center for different thermal expansion factors........ 58

Figure 4.23. Flame velocity at the channel center for different thermal expansion. .................. 59

Figure 4.24. Flame velocity in channels of half width $5 L_{f}$, at two different wall temperatures. . 60

Figure 4.25. Flame velocity in channels of half width $15 L_{f}$, at two different wall temperatures. 60 
Figure 4.26. Flame velocity at the center of the channel, $R_{c h}=30 L_{f}$, at two wall temperatures.

Figure 4.27. Flame propagation regimes as described by the channel wall temperature and width. 62

Figure 4.28. Flame propagation regimes as described by the channel wall temperature and width.

Figure 4.29. Flame propagation regimes as described by the channel wall temperature and width.

Figure 4.30. Flame propagation regimes described by the scaled width and thermal expansion coefficient. 65

Figure 4.31. Flame propagation regimes described by the scaled width and thermal expansion. 66 Figure 4.32. Flame propagation regimes described by the scaled width and thermal expansion. 66 Figure 4.33. Flame propagation regimes in adiabatic conditions [63] 67

Figure 4.34. Flame velocities in tubes and channels with adiabatic and isothermal conditions... 68 Figure 4.35. Flame velocities in tubes and channels with two different isothermal conditions... 68

Figure 4.36. Flame velocities in channels at later stages, $\Theta=8, R_{c h}=10 L_{f}, T_{w}=300 \mathrm{~K}$ [64].69 Figure 4.37. Flame propagation including later stages. $T_{w}=300 \mathrm{~K}, \Theta=8, R_{c h}=20 L_{f}$. a) $\tau_{f}=14.5$, d) $\tau_{f}=22.8$, e) $\tau_{f}=25.4$, f) $\tau_{f}=26.6$, g) $\tau_{f}=30.4$ 70

Figure 4.38. Description of the flame propagation in a channel of width $2 R_{c h}$, where obstacles of length $\alpha R_{c h}$ have been included, considering isothermal wall conditions. 71

Figure 4.39. Flame velocities obtained in obstructed channels with different blockage ratios... 72

Figure 4.40. Flame tip velocities obtained in obstructed channels with different $\alpha . R_{c h}=20 L_{f} 73$ Figure 4.41. Flame tip velocities obtained in obstructed channels with different $\alpha, R_{c h}=30 L_{f} 74$

Figure 4.42. Evolution of the flame tip position in a channel considering different widths. ....... 75 Figure 4.43. Flame velocities in obstructed channels, considering different obstacle separations.

Figure 4.44. Flame velocities in obstructed channels, considering different obstacle separations. 77

Figure 4.45. Flame velocities in obstructed channels, considering different obstacle separations. 77 
Figure 4.46. Temperature distributions in obstructed channels (upper half shown), $R_{c h}=24 L_{f}$, $\alpha=2 / 3$, and different spacing a) $\Delta Z / R_{c h}=0.38$, b) $\Delta Z / R_{c h}=0.6$ and c) $\Delta Z / R_{c h}=1.78$

Figure 4.47. Flame tip velocities obtained in obstructed channels considering different wall thermal conditions: isothermal conditions (solid) and adiabatic conditions (dashed).......... 79

Figure 4.48. Flame tip velocities in a channel considering different wall temperatures. ............ 80

Figure 4.49. Instantaneous temperature distributions and streamlines describing the flame propagation in an obstructed channel given by $R_{c h}=20 L_{f}, \alpha=1 / 2, \Delta Z / R_{c h}=0.2$,

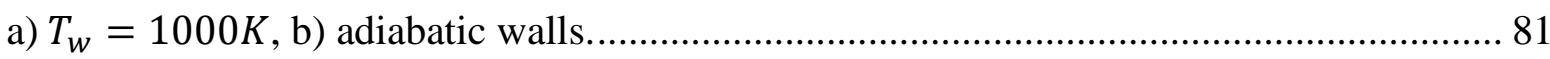

Figure 4.50. Flame tip velocities obtained in a channel considering small blockage ratios........ 82 Figure 4.51. Temperature distribution describing the finger flame limit in obstructed channels $R_{c h}=24 L_{f}, \alpha=1 / 20, \Delta Z / R_{c h}=0.6$. a) $\tau=0.228$, b) $\tau=0.287$, c) $\tau=0.351$, d) $\tau=$ 0.386 83

Figure 4.52. Flame velocities calculated numerically and analytically (Eq. 24), $\Delta Z / R_{c h}=0.38$.

Figure 4.53. Flame velocities calculated numerically and analytically (Eq. 24 ), $\Delta Z / R_{c h}=0.6 .86$ Figure 4.54. Temperature distributions and streamlines describing laminar and turbulent flame propagation regimes. $R_{c h}=24 L_{f}, \Delta Z / R_{c h}=0.6$, a) $\alpha=1 / 5$, b) $\alpha=1 / 2 \ldots \ldots \ldots \ldots \ldots \ldots . .86$

Figure 4.55. Flame tip velocities found numerically and analytically (Eq. 24) for various $\alpha \ldots \ldots 87$ Figure 4.56. Temperature distributions and streamlines describing the laminar and turbulent regimes obtained in obstructed channels. $R_{c h}=24 L_{f}, \Delta Z / R_{c h}=1$, a) $\alpha=1 / 5$, b) $\alpha=1 / 2$ 88

Figure 4.57. Flame regimes in obstructed channels. Analytical prediction given by Eq. 26. ..... 89 Figure 4.58. Flame tip position obtained in obstructed (solid) and in 'smooth' channels (dashed). Black: $\alpha=2 / 3, W_{c h}=16 L_{f} ;$ red: $\alpha=1 / 2, W_{c h}=24 L_{f}$ and blue: $\alpha=1 / 40, W_{c h}=$ $46.8 L_{f}$ 90

Figure 4.59. Flame tip position obtained in obstructed (solid) and in 'smooth' channels (dashed). Black: $\alpha=2 / 3, W_{c h}=16 L_{f}$; red: $\alpha=1 / 2, W_{c h}=24 L_{f}$ and blue: $\alpha=1 / 40, W_{c h}=$ $46.8 L_{f}$ 91

Figure 4.60. Comparison of the flame acceleration produced by obstructed and frictional wall. 92 


\section{List of Symbols}

$C_{V} \quad$ Heat capacity at constant volume.

$C_{P} \quad$ Heat capacity at constant pressure.

c Speed of the sound.

$E_{a} \quad$ Activation energy.

$L_{f} \quad$ Flame thickness.

$L_{f, w} \quad$ Flame thickness, including wall effects.

$L_{c} \quad$ Cell length.

m Constant molar mass.

Ma Mach number.

$P \quad$ Pressure.

Pr Prandtl number.

$Q \quad$ Specific energy released in a chemical reaction.

$r \quad$ Radial position.

$R \quad$ Flame tip position along the conduit.

$R_{c h} \quad$ Half width of the channel.

$R_{c} \quad$ Flame tip position at the conduit centerline.

Re Reynolds number.

$R_{p} \quad$ Universal gas constant.

$R_{t u} \quad$ Tube radius.

Sc Schmidt number.

T Temperature.

$T_{w} \quad$ Wall temperature.

$t \quad$ Time.

$u \quad$ Velocity component.

$U_{c} \quad$ Flame velocity at the center of the pipe.

$U_{f} \quad$ Planar flame velocity. 
$U_{t} \quad$ Flame tip velocity.

$U_{w} \quad$ Flame velocity near the wall.

$Y \quad$ Mass fraction of the fuel.

Z Axial position.

Greek Letters

$\alpha \quad$ Blockage ratio.

$\gamma \quad$ Adiabatic exponent.

$\varepsilon \quad$ Total energy per unit volume.

$\zeta \quad$ Stress tensor.

$\eta \quad$ Dynamic viscosity.

$\Theta \quad$ Thermal expansion coefficient.

$\lambda \quad$ Cell width size.

$\rho \quad$ Density.

$\sigma \quad$ Acceleration rate.

$\tau \quad$ Scaled time.

$\tau_{f} \quad$ Scaled time with respect to the flame.

$v \quad$ Kinematic viscosity. 


\section{Introduction}

Combustion is a chemical reaction where oxidation of a fuel takes place. This oxidation event, however, has a component that makes it different from other oxidation reactions: heat is released during the process, raising the fuel temperature and facilitating the reaction, making combustion a self-sustained process.

Combustion of fossil fuels is largely the main source of power generation in the present time [1]. In spite of the continuously increasing usage of renewable energy sources, projections suggest that liquid fuels, coal and natural gas will represent more than $75 \%$ percent of the energy sources in 2040, as illustrated in Figure 1.1 [1]. Applications of combustion-related technologies can be found in numerous fields such as industry (steam production, metallurgy) and propulsion systems (vehicles, airplanes, rockets). In this sense, the number of problems associated with its operation is also significant: environmental damage as a result of the products of combustion, safety issues due to uncontrolled burning, lack of fuel availability necessary to keep the pace of current energy consumption, etc. This situation demands higher standards of burning efficiency and control, which relies on a better understanding of the combustion phenomenon.

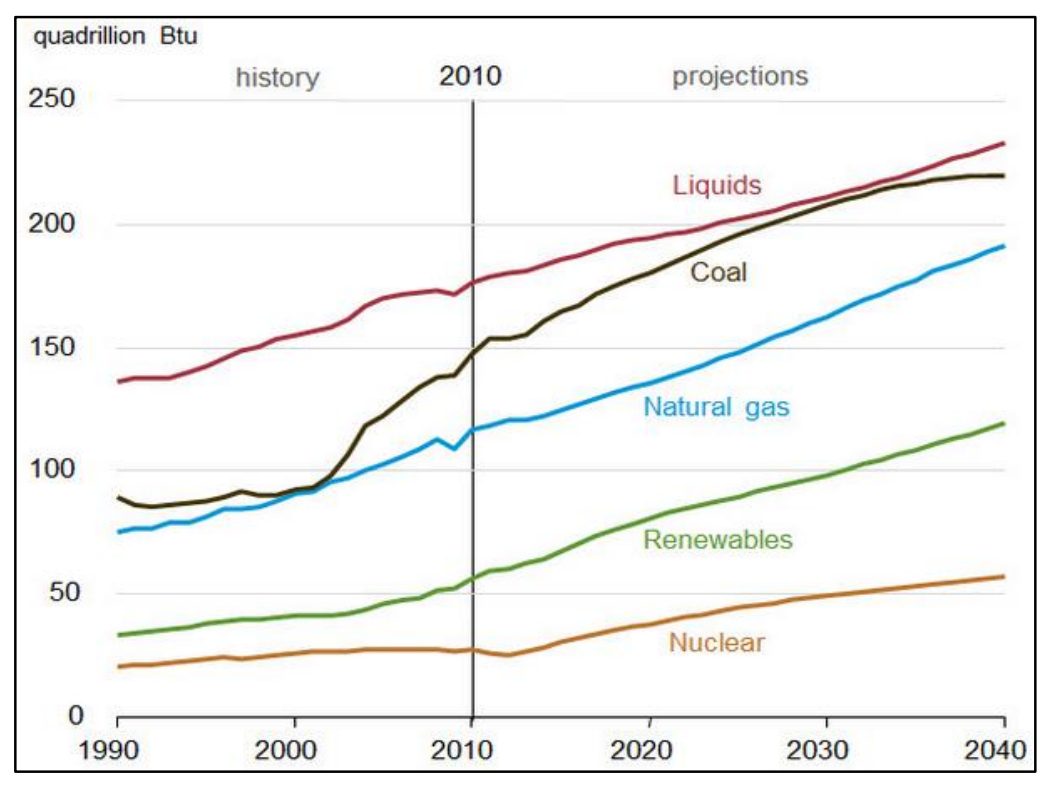

Figure 1.1 World energy consumption and prediction by fuel type, 1990-2040 [1]. 
From the scientific point of view, combustion is one of the most challenging topics to deal with. The oxidation of the fuel occurs through a chain of a large number of chemical reactions and physical mechanisms, each one associated with a particular time and length scale. Moreover, the interaction of the generated flow with the chemistry component of the event can be attained at either laminar or turbulent conditions, determining substantial changes on the burning process.

Combustion can be classified according to how the fuel and the oxidizer are arranged before reacting in: premixed and non-premixed combustion. Premixed combustion is characterized by the mixing of the fuel and the oxidizer before the ignition; spark ignition engines are one of the most known examples of this combustion type. In non-premixed combustion, the fuel and the oxidizer remain separated before entering the burning zone, the mixing of gases is given by molecular or turbulent diffusion only, the Diesel engine being one of the most used applications of this type.

\subsection{Flame Dynamics in Premixed Combustion}

In the present work, the attention is restricted to premixed combustion. The self-sustained mechanism in this case is illustrated schematically in Figure 1.2.

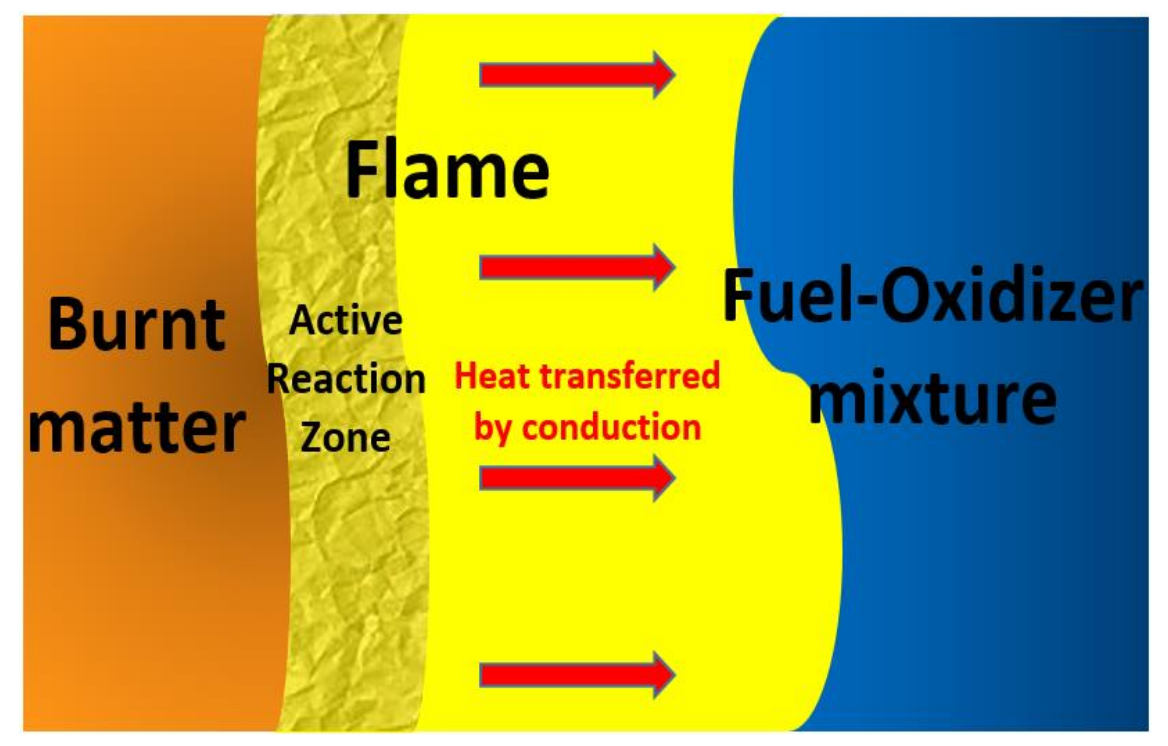

Figure 1.2. Illustration of the mechanism of premixed flame propagation.

As observed in Figure 1.2, the heat released in the reaction zone is transferred to the nearby fueloxidizer mixture by thermal conduction. As a result, the mixture is heated and ignited after 
reaching a certain temperature, also releasing heat to the surrounding and carrying the flame towards the fresh fuel mixture. The burning region, including the active reaction zone, where heat and light are released, constitutes the flame. This type of combustion shows a remarkable dependence of the reaction rate on temperature, e.g. increasing the fuel temperature by a factor of two might lead to reaction rates 10-12 orders of magnitude larger.

In premixed combustion, the comparison of the attained flame propagation velocity to the speed of sound allows the following sensitive distinction:

1) Deflagration: the flame propagation velocity in this case is less than the speed of the sound, with typical values around $1 \mathrm{~m} / \mathrm{s}$ [2]. In this case, the reaction is driven by thermal conduction.

2) Detonation: characterized by a fast, supersonic regime, with typical flame propagation velocities around $2000 \mathrm{~m} / \mathrm{s}$ [2]. In this case, the reaction proceeds due to shock waves, compressing and preheating the fuel mixture, determining conditions for the combustion reaction away from the flame front.

The typical propagation velocities characterizing these regimes show that the detonation front propagates two to four orders of magnitude faster than that of the deflagration. Under certain conditions, spontaneous acceleration of a propagating flame results in a deflagration-todetonation transition (DDT) event. The DDT is a fundamental combustion problem, considered one of the most relevant unsolved problems in combustion science [2]. The underlined differences between the deflagration and detonation regimes make DDT a powerful phenomenon, very dangerous in those scenarios where it has not been predicted or controlled yet (e.g. accidental explosions observed in mines), but with a great technological potential, as currently observed in pulse detonation engines.

Some concepts of the flame dynamics can be learned from Figure 1.3. In there, results of the analysis done by Zeldovich and Frank-Kamenetski [3] are shown, where the flame is assumed to propagate keeping a planar shape. The temperature, density, flow velocity and fuel mass fraction distribution have been scaled by the fuel and burned matter temperatures $\left(T_{f}\right.$ and $\left.T_{b}\right)$, burned matter density $\left(\rho_{b}\right)$ and the laminar flame velocity $\left(U_{f}\right)$, respectively, along the domain defined 
by the scaled position $z / L_{f}$, where $L_{f}$ is the flame thickness, conventionally defined as $L_{f}=$ $v / U_{f} P r$.

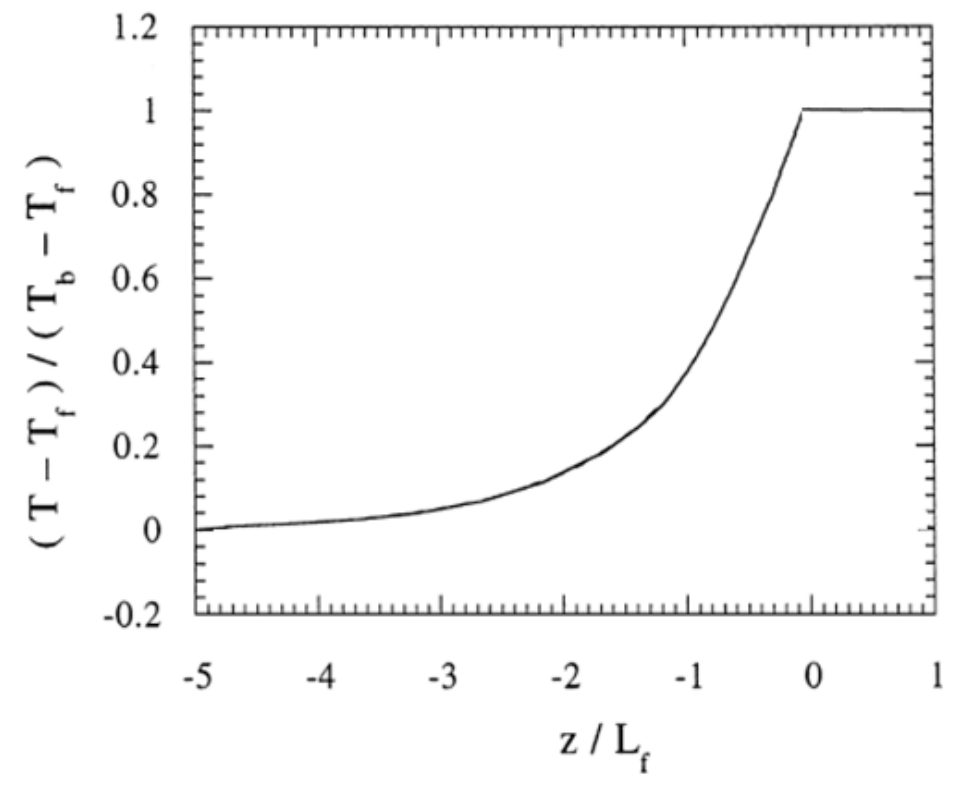

a)

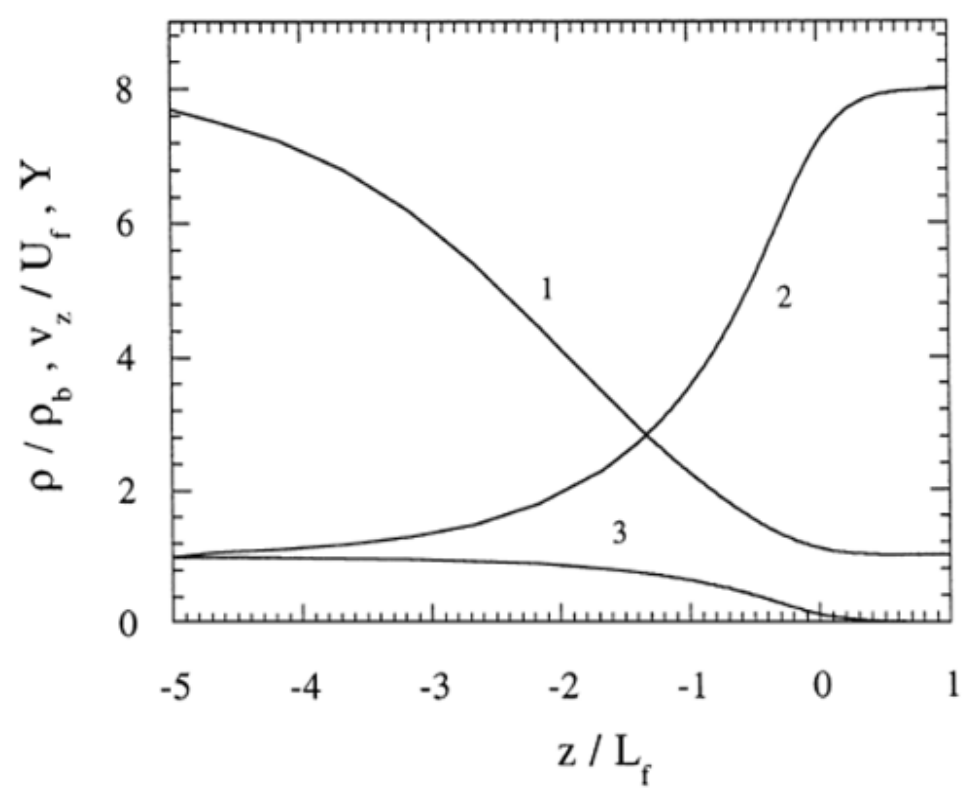

b)

Figure 1.3. a) Scaled temperature of a planar flame propagation. b) Distribution of the scaled density (1), flow velocity (2) and fuel fraction (3) [4]. 
The temperature profile shows the temperature increase associated with the energy released at the reaction zone, around $z / L_{f}=0$ (top). The flame propagation is driven by the thermal expansion experienced by the burning matter (characterized by the factor $\Theta$ ), with the density and flow velocity distributions showing a temperature dependence given by $\rho=\rho_{f} T_{f} / T$ and $v_{f}=U_{f} T / T_{f}$ (bottom). The heat transferred to the fresh mixture determines the smooth transition observed in the vicinity of the reaction zone, with exception of the temperature distribution case, where a step function is considered:

$$
T(z) / T_{f}= \begin{cases}1+(\Theta-1) \exp \left(z / L_{f}\right), & z<0 \\ \Theta, & z>0\end{cases}
$$

This analytical description does not apply if more realistic elements are taken into consideration, e.g. flame fronts experiencing corrugation, which produce faster flame propagation than planar flames as a result of the larger flame surface area attained.

\subsection{The Importance of Micro Combustion}

In the recent time, some of the most important technology applications and manufacturing development have been moving toward smaller length scales. Examples of them include miniature satellites and unmanned air vehicles, which are used for the monitoring of diverse activities such as traffic flow [5], agriculture [6], environment [7], etc., besides their original military purpose.

In this sense, it should be addressed that the development of such new technologies is limited by its power source, which should be able to fit not only its geometry needs, but typical power source characteristics such as been long-lived, inexpensive, safe, etc. This situation makes combustion at small length scales very important, since the energy densities of hydrocarbon fuels are much greater than, for example, batteries - the only alternative at present when the device size is further reduced (Figure 1.4). The large energy density of fossil fuels has conveyed an associated advance in fabrication techniques of micro electro-mechanical systems (MEMS), Figure 1.5, although the building of a working MEMS engine has not been possible up to date, that the author is aware of. 


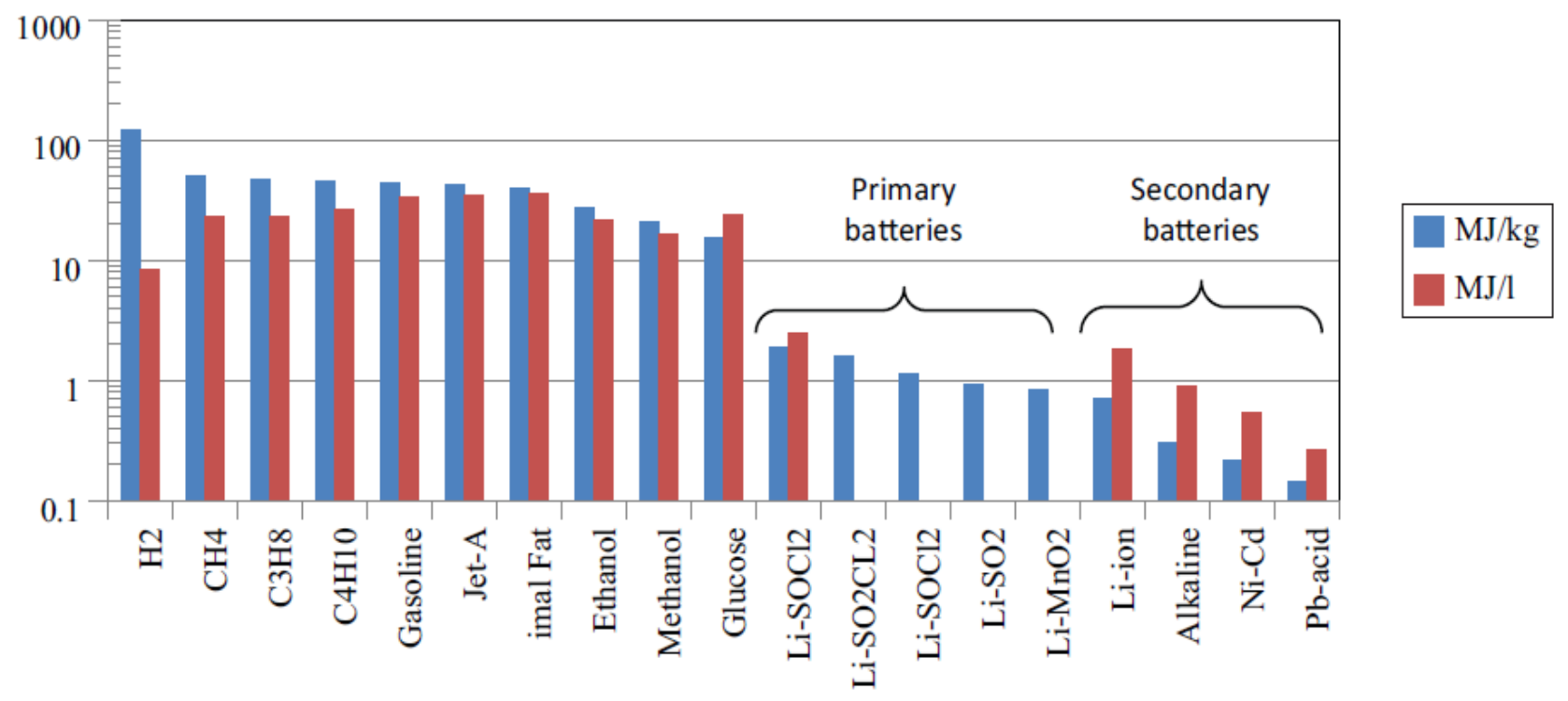

Figure 1.4. Gravimetric and volumetric energy densities of diverse materials [8-14]
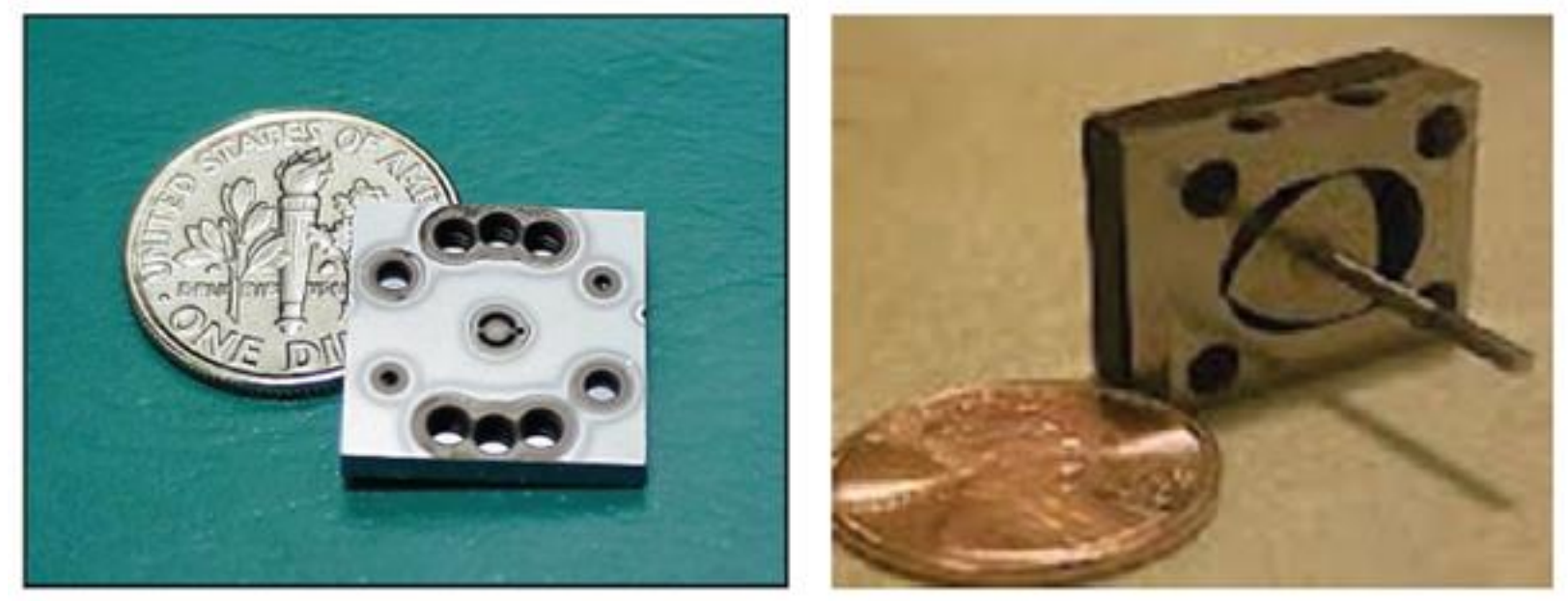

Figure 1.5. Micro turbine rotor [15], left. Rotary internal combustion engine [16], right.

However, fabrication is not the only problem when developing MEMS engines. The combustion process in small scales also provides a wide range of difficulties and problems to solve. For example, according to the 'cube-square' law [17], the power system should be able to generate more power per unit mass as the length scale of the device is reduced. Namely, considering a constant air mass flow in the power system, the generated power depends basically on this flow value, since the burning process determined by chemical reactions is scale-independent. The flow varies with the cross section area, and the system mass with the system volume, which 
yields an inverse proportionality between the power density and the system length scale, specifically: Power $_{\text {sys. }} /$ mass $_{\text {sys. }} \propto A_{\text {cross section }} / V_{\text {sys. }} \propto 1 / L$, where $L$ is the system length scale. Therefore, the power density should increase as the system size is reduced. In reality, however, keeping the air flow constant becomes more difficult as the engines get smaller, due to the larger effect of the fluid friction, heat losses and radical quenching at the surfaces. Figure 1.6 [8] shows the power density of a simplified tubular combustor in relation to its length scale, where the tendency provided by the 'cube-square' law and that including convective losses are compared, along with the thermal efficiency.

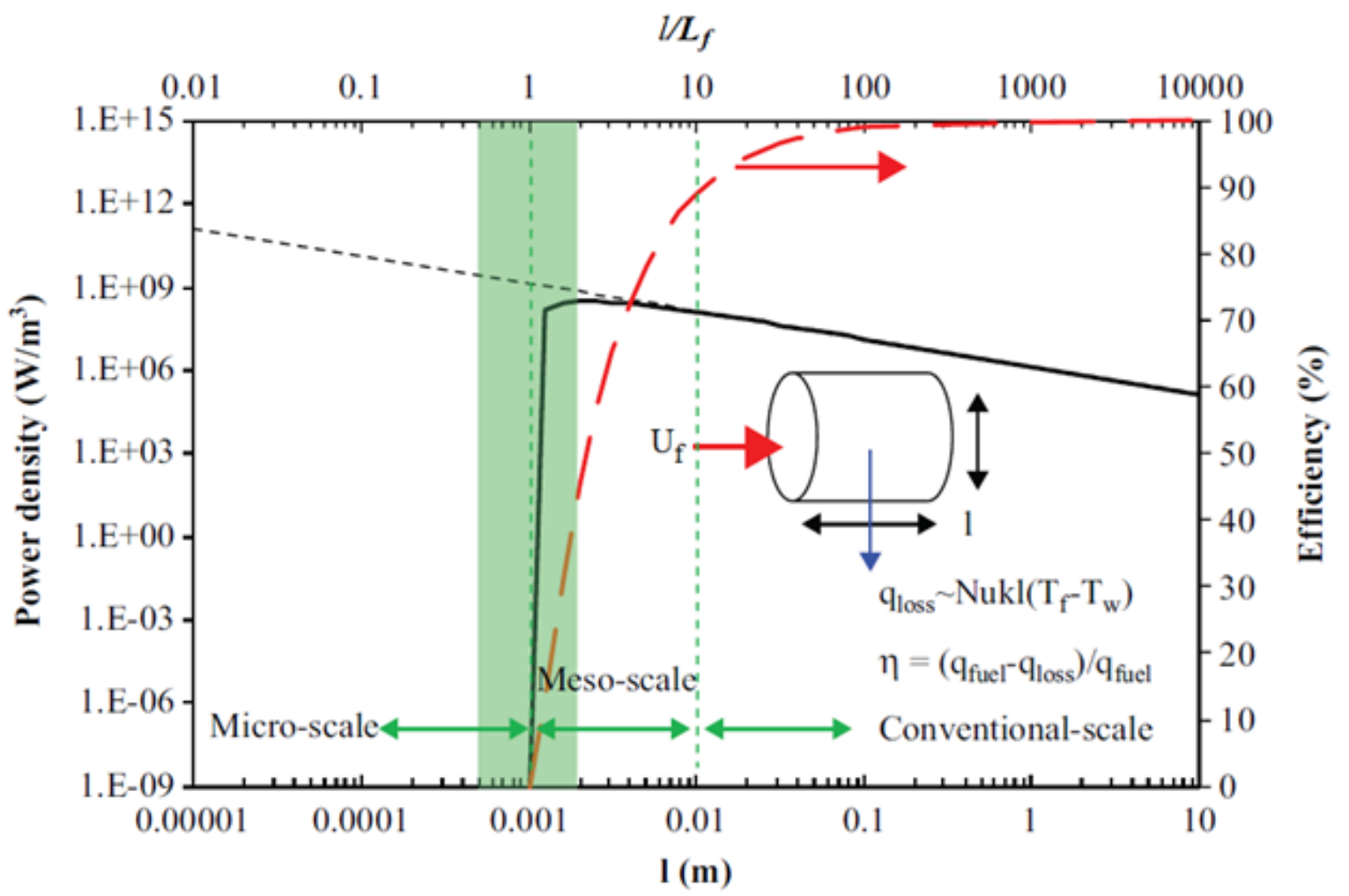

Figure 1.6. The power density, calculated by the 'cube-square' law (dotted) and by including convective losses (solid), and efficiency (dashed) of a simplified combustor are shown in relation to its length scale (1) and freely propagating flame thickness $\left(L_{f}\right)$. Results are based on a fuel with specific energy value of $44 \mathrm{MJ} / \mathrm{kg}$, laminar flame speed $U_{f}=42 \mathrm{~cm} / \mathrm{s}$, and flame thickness $L_{f, w}=1 \mathrm{~mm}[8]$.

The power density predicted by the 'cube-square' law holds until the combustor length reaches the meso-scale region, which is characterized by heat loss rates comparable to the heat generated. 
Moreover, the efficiency curve shows a dramatic reduction around this region, including a thermodynamically inviable condition as the combustors size is further decreased. Although results of Figure 1.6 are not quantitatively accurate, due to the simplifications considered, it does show the importance of understanding the heat and momentum transfer to the combustor boundaries in order to achieve efficient and reliable power micro-combustion systems. The necessity is related to the developing of materials that can overcome these limitations as well as a better understanding of the combustion process and flame morphology at the conditions found in micro-scale and meso-scale combustors. The present investigation is focused on the latter.

\subsection{The Scope of this Investigation}

In this study, the effects of the chamber boundary conditions on the flame propagation dynamics are explored. For this purpose, a parametric study has been performed by numerical means, which includes two chamber configurations:

1) Channels and tubes with nonslip walls, subjected to adiabatic and isothermal conditions. In this configuration, the flame propagation and morphology are explored in relation to the wall thermal conditions, comparing the effect of multiple constant wall temperatures on the flame propagation, including the adiabatic case. These effects are investigated regarding the energy released by the fuel mixture during the combustion process, characterized by the fuel thermal expansion.

2) Obstructed channels, considering adiabatic and isothermal surfaces as well. The flame propagation is investigated in relation to the obstacles-channel geometry, determining its influence on the flame dynamics attained in the early stage of the process. A comparison between the flame acceleration obtained in this configuration and the produced in the previous 'smooth' conduits is also considered.

This manuscript has been organized as follows: Chapter 2 presents a literature review, where theory relevant to this investigation is described; Chapter 3 presents the methodology, including the set of hydrodynamics and combustion equations solved numerically, a description of the grid domain and boundary conditions of the two configurations investigated, as well as the validation

of the numerical results; Chapter 4 presents the results of the parametric study, and Chapter 5 the conclusions and future work sections. 


\section{Literature Review}

The deflagration-to-detonation transition (DDT) can be generated by an intense expansion of the burning mixture which, acting as a piston, promotes a spontaneous reaction in the fresh fuel ahead of it [18]. Zeldovich et al. [19] described the DDT in tubes as a sequence of stages, starting with the acceleration of the flame front. This accelerated front pushes weak shocks, which after interacting between them, are combined into stronger shock waves that compress and heat the fresh unburnt mixture ahead of the flame front. This generates an explosion in the region limited by the flame front and the leading shock, producing detonation. The process is illustrated schematically in Figure 2.1.

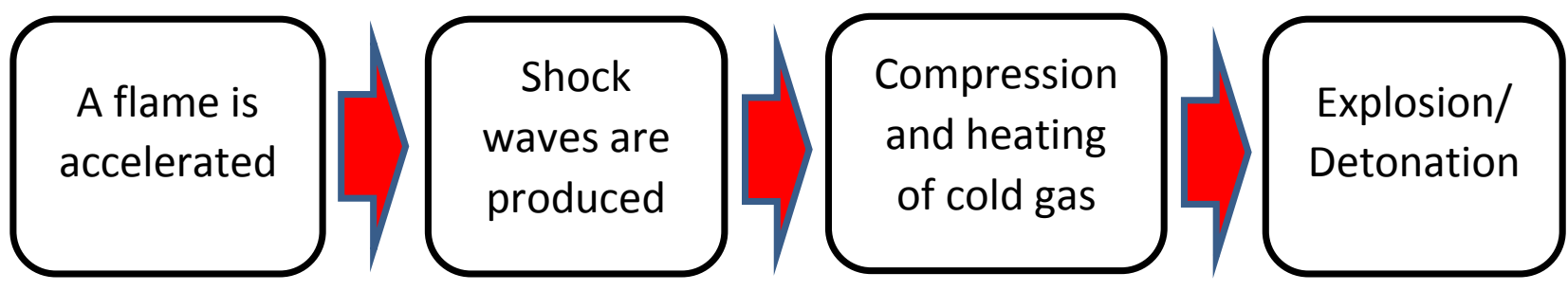

Figure 2.1. The DDT stages as described by Zeldovich et al. [19].

Attempts to scrutinize the DDT scenario have inspired a continuous research of the mechanisms that accelerate the propagation of the flame front, which is the onset of the whole process described above. From experimental observations in tubes, where DDT was first observed, to numerical studies, where the DDT stages were further revealed, a big effort has been devoted to improve the understanding of these mechanisms. Thus, spontaneous acceleration observed in tubes have been related to flame instabilities, turbulence, acoustic effects and the features of the geometric configuration of the chamber and wall conditions. In the following sections, a brief review of the studies related to these mechanisms is presented.

\subsection{Effect of Turbulence on the Flame Dynamics}

The burning process is coupled to the flame shape in such a way that larger flame front areas provide faster burning rates. Therefore, a turbulent flow interacting with a burning domain will increase the flame propagation velocity by corrugating (and increasing) the flame surface, leading to flame acceleration. 
In spite of the intrinsic difficulties that turbulent flow possesses, it has been possible to establish, at least qualitatively, different regions of the interaction of the combustion process with the external turbulent flow. These regions are presented in the Barrere-Borghi diagram, Figure 2.2, in terms of the turbulent intensity $U_{\text {turb }}$ (the root-mean-square velocity in one direction) and the integral turbulent length $L_{\text {turb }}$, scaled by the planar flame velocity, $U_{f}$, and the flame thickness, $L_{f}$, respectively.

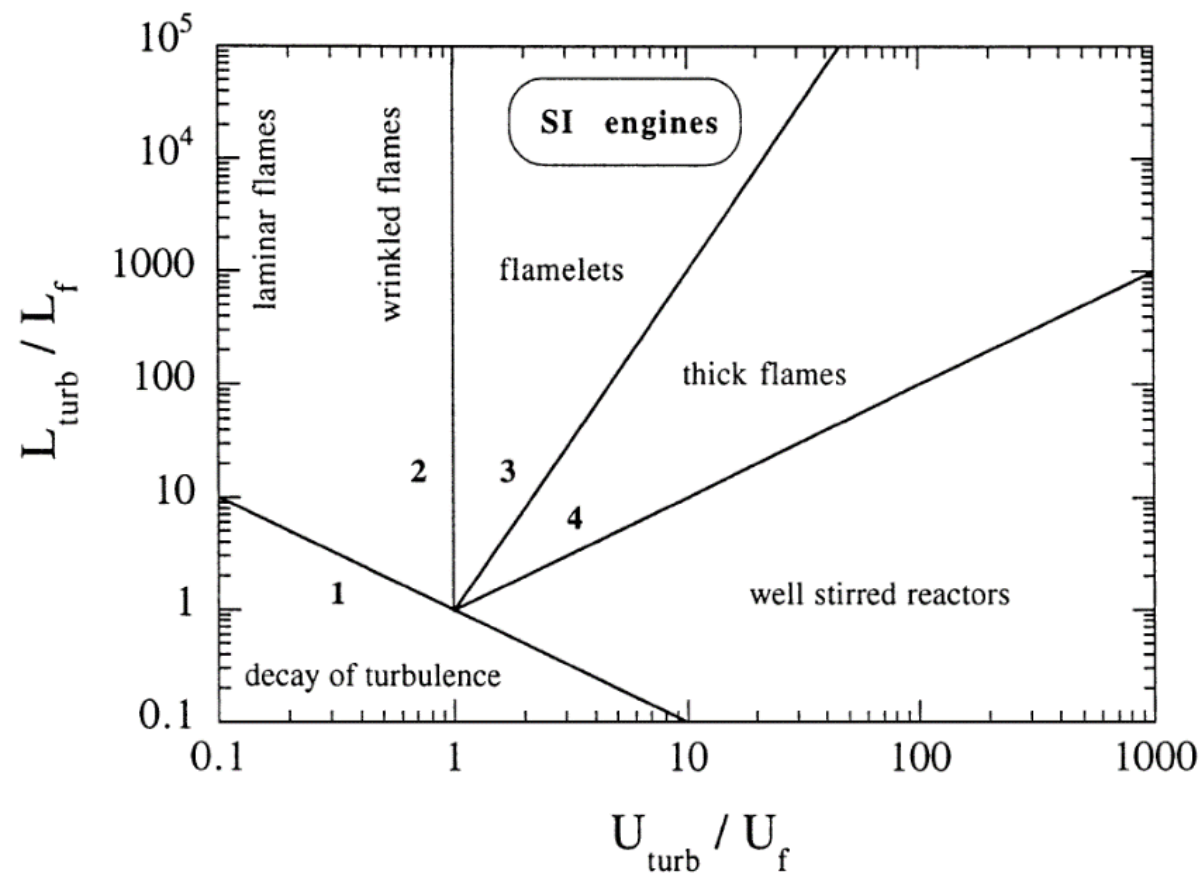

Figure 2.2. Regimes of turbulent combustion [4].

Region 1 is related to low Reynold numbers $R e=U_{\text {turb }} L_{t u r b} / v<1$, which indicates a decaying turbulent regime. By increasing the length scale of the flow (but keeping low turbulent intensity) Region 2 is approached, where a transition from a laminar to a wrinkled flame can be noticed. Although a turbulent combustion regime is attained here, it is not responsible for the corrugation of the flame which occurs due to the flame instabilities. In Region 3, which is associated with turbulent flamelets, the turbulent flow corrugates the flame shape strongly at large length scales; however, the internal structure and transport properties remain laminar. In terms of practical applications, this is the most interesting regime, since it is the typical scenario for car engines and gas turbines operation. Region 4 is characterized by a more intense turbulence flow, which is able to modify the transport coefficients after penetrating the internal flame structure, generating 
a thicker flame. Finally, in the well stirred reactor regime, a very intense turbulent flow is obtained, where the burning zone does not exhibit a "front" anymore.

\subsection{The Darrieus-Landau Instability}

Darrieus (1938) [20] and Landau (1944) [21] investigated the hydrodynamic instability of premixed flame fronts in gaseous fuel mixtures. This instability is driven by the thermal expansion resulted from the exothermal reaction. The analysis established that an infinitely thin flame front would be absolutely unstable against any perturbations on it. Consequently, at a linear stage, the flame corrugations will grow exponentially in time, distorting the flame front and accelerating it, as the reaction surface area is enlarged.

Pelce and Clavin [22] extended this analysis to flames of finite thickness, noticing the thermal stabilizing role that the internal flame structure plays on the development of the Darrieus-Landau instability. The mechanism is illustrated schematically in Figure 2.3, namely, heat fluxes within the flame front of thickness $L_{f}$ converge to the convex regions, making them warmer and thereby accelerating their propagation. In contrast, heat fluxes towards the concave regions diverge, moderating their propagation. Cumulatively, this reduces the flame surface area, playing a stabilizing role against the Darrieus-Landau instability.

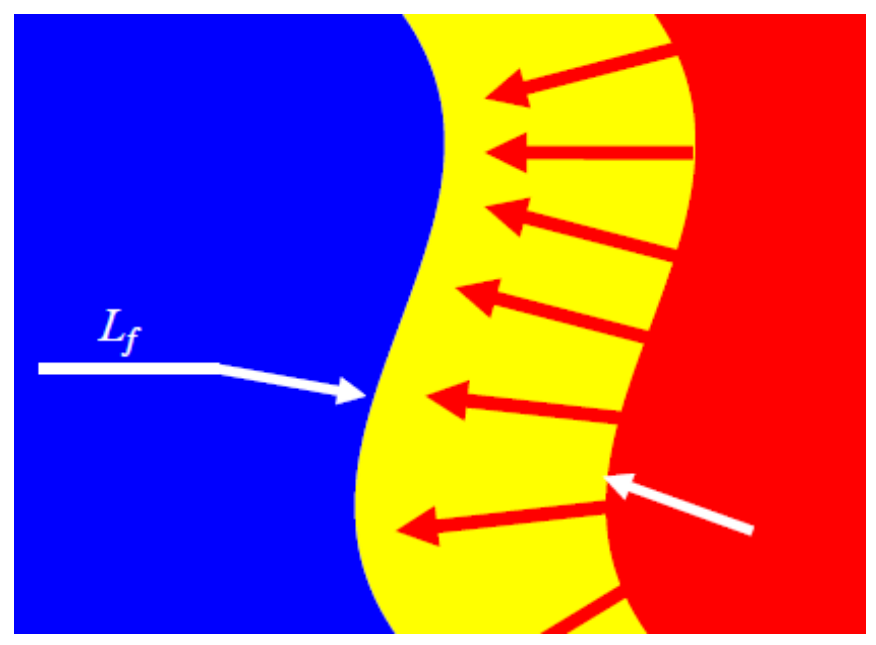

Figure 2.3. Thermal stabilization of the Darrieus-Landau instability. Courtesy of V. Bychkov.

This analysis leads to the concept of a cutoff (threshold) length scale, associated with the Darrieus-Landau instability. Specifically, a planar flame front can propagate in tubes of width smaller than the cutoff length scale, given by the instability linear theory. When the width 
exceeds this cutoff value, the flame becomes curved and propagates faster in a steady or quasisteady manner [23, 24]. Clavin and Garcia [25] obtained a complete derivation of the linear dispersion relation, including the effects of diffusion varying with temperature and direction, viscosity, equations of state and other transport coefficients. Kobayashi and Kawazoe [26] observed that the effect of the Darrieus-Landau instability leading to flame front acceleration is possible in the absence of rigid walls, obstacles or other significant perturbations, at large Reynolds numbers $\left(10^{3}-10^{5}\right)$.

However, when including limited hydrodynamic length scales (as in tubes and channels), and when considering realistic density drop at the flame front, the flame acceleration generated by this mechanism is too weak, and the associated time scale is too short to trigger detonation [27].

\subsection{Flame-Acoustic Interactions}

Closed chambers provide conditions for flame-acoustic interactions. In these geometries, very common in industrial applications, the flame dynamics and the combustion instabilities are coupled to the imposed and flame-generated pressure waves. In this sense, it is possible to distinguish two types of pressure waves: weak shocks, if the time needed for the sound to traverse the chamber is longer than the characteristic flame dynamics time; and acoustic waves if it is smaller [4].

The effect that the pressure waves have on the flame dynamics can be understood, for example, from the experimental results obtained by Searby [28]. In this work, the experimental setup generated a flame propagating from an open top to a closed bottom of a vertical tube. It was noticed that the flame propagation was conditioned by the flame velocity, namely, for flame velocities less than $16 \mathrm{~cm} / \mathrm{s}$, the propagation included no sound, and the flame shape remained almost planar as a result of the gravity stabilizing effect. By increasing the flame velocity, $16 \mathrm{~cm} / \mathrm{s}<U_{f}<25 \mathrm{~cm} / \mathrm{s}$, the flame became curved at the upper part of the tube as a result of the Darrieus-Landau instability, producing a further increment on the flame propagation velocity and generating sound waves.

When faster flame velocities were considered, $25 \mathrm{~cm} / \mathrm{s}<U_{f}<40 \mathrm{~cm} / \mathrm{s}$, cellular structures appeared at the flame front, close to the tube end. These cellular structures oscillated faster than the acoustic period, proving to be a consequence of the parametric instability regime of the flame 
front in a field generated by the acoustic waves. The sound wave amplitude, in turn, was strongly increased, confirming the coupling of the flame to sound waves. Finally, faster flame velocities resulted in a transition to a turbulent regime, and a consequent velocity increase (up to $750 \mathrm{~cm} / \mathrm{s}$ ).

\subsection{The Finger Flame Model}

Clanet and Searby [29] presented a set of experiments where it was possible to identify another flame acceleration mechanism, the so-called 'finger flame' model. The idea was based on observations of flame propagation in tubes with an anisotropic character in the early stages. The experimental setup included a tube with one end open and the other closed, ignited at the intersection of the closed end and the tube axis. The flame propagation approached a finger shape (Figure 2.4), with four distinctive stages. First, the flame propagates at a constant velocity with a hemispherical shape. Second, the propagation develops faster in the axial direction, forming a finger shape on the flame front. Third, the finger flame acceleration stops when the 'skirt' of the flame reaches the side wall. Fourth, the flame decelerates, it turns into an almost planar shape and eventually becomes the so-called 'tulip flame'. Although the acceleration rate obtained in this process was quite high, it lasted for a very short time.

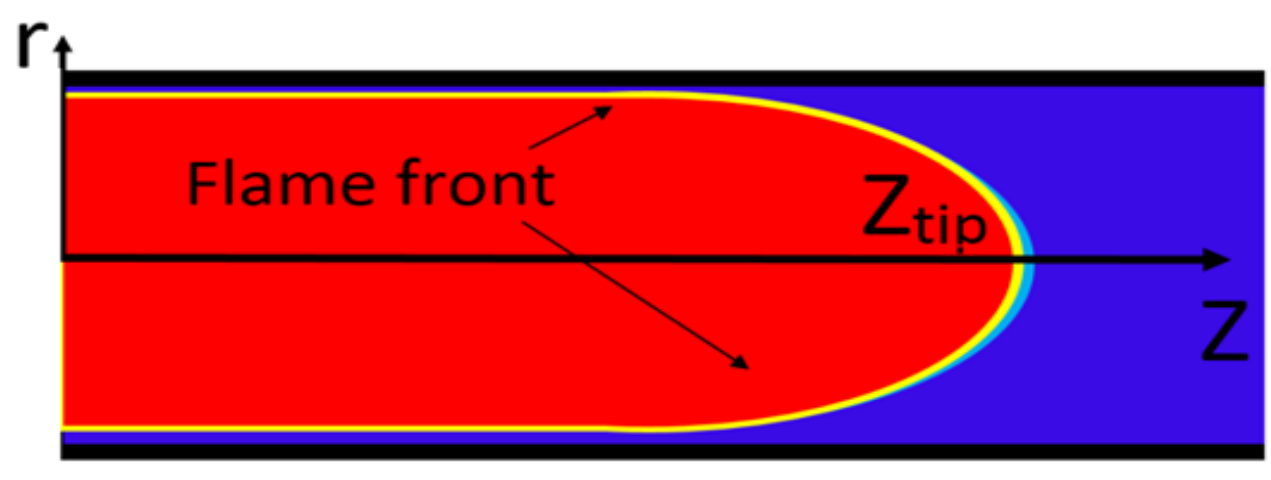

Figure 2.4. 'Finger' flame model of flame propagation.

Bychkov et al. [30] developed an analytical formulation for the finger flame model, determining the growth rate, time scales of the different stages and increment of the flame surface area. Numerical results included in the study also showed the deceleration stage with the occurrence of the "tulip flame" structure. As observed in [29], the acceleration phase described by this flame propagation was found to be very short in time, vanishing once the flame reaches the tube walls and restricting this acceleration mechanism to the early stages of burning only. The analytical expressions for the characteristic time scales of the different stages were proposed as follows: 
a) The time at which a hemi-spherical flame shape is replaced by a finger form:

$$
t_{\text {sphere }} \approx R_{f} / 2 \phi U_{f},
$$

where $R_{f}=\Theta U_{f} t$ is the radius of the flame front, $\Theta$ the thermal expansion coefficient, $U_{f}$ the planar flame velocity and $\phi=\sqrt{\Theta(\Theta-1)}$.

b) The time when the 'skirt' of the flame touches the wall:

$$
t_{\text {wall }}=\frac{R_{\text {skirt }}}{2 \phi U_{f}} \ln \left(\frac{\Theta+\phi}{\Theta-\phi}\right),
$$

with $R_{\text {skirt }}=\left(\Theta R_{f} / \phi\right) \tanh \left(\phi U_{f} t / R_{f}\right)$ being the radius of the flame front.

c) The formation of the 'tulip flame':

$$
t_{\text {tulip }}=t_{\text {wall }}+t_{i n v}
$$

where $t_{i n v}=\sigma^{-1} R_{f} / U_{f}, \quad \sigma=2 \phi=2 \sqrt{\Theta(\Theta-1)}$ is the flame acceleration growth rate identifying the exponential increase in the corrugated flame speed $U_{w} \propto \exp \left(\sigma U_{f} t / R\right)$. A comparison of this formulation to the experimental results of Clanet and Searby [29] is shown in Figure 2.5.

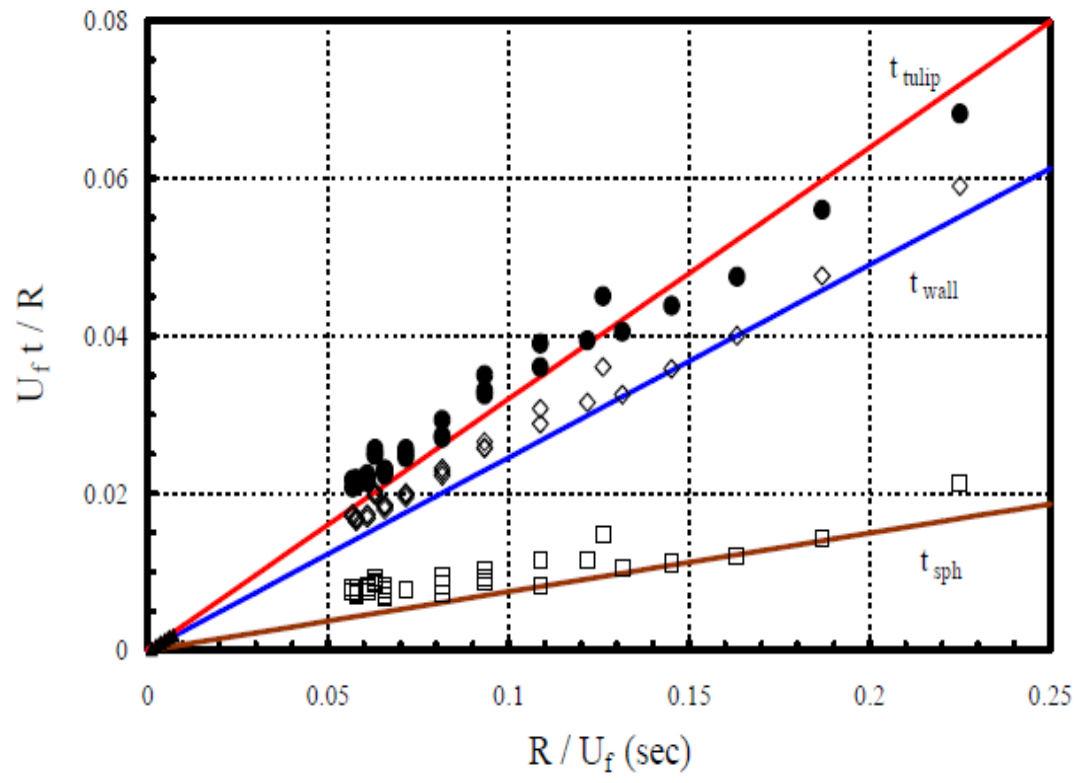

Figure 2.5. Comparison of the time scales of the flame propagation stages observed in finger flames, as predicted analytically (solid) and found experimentally (markers) [30]. 


\subsection{The Shelkin Mechanism of Flame Acceleration}

An explanation of the spontaneous flame acceleration observed in tubes was suggested by Shelkin [31]. It was proposed that as the flame front propagates from a closed tube end, a flow in the fresh mixture is generated by the thermal expansion of the burning matter. The flow is restricted at the walls by frictional forces therein and, as a result, the flame front area is enlarged, intensifying the combustion and accelerating the flame front.

For a long time, the developing of a theory out of Shelkin's work was not possible, since the flame acceleration attained by this mechanism generates turbulent flow regimes, which along with the reacting character of the process, provides a very difficult problem, currently unsolved (see some details in Sec. 2.1).

\subsubsection{The Laminar Flame Approximation}

In an investigation conducted in 2000, Brailovsky and Sivashinsky [32] described the DDT events by using quasi-one-dimensional model combustors. With the help of rough tubes, they suggested that the compression and preheating of the fresh mixture near the propagating flame front, caused by hydraulic resistance at the walls, may produce localized thermal explosions. Later, Kagan and Sivashinsky [33] proposed that the DDT could be explored in laminar environments. In this sense, the authors considered a two-dimensional channel with adiabatic walls, keeping the width thin enough to ensure that only laminar flows were generated. The results showed that, in addition to the previous detonation mechanism, an accelerating tulip flame was formed which promotes a secondary detonation. Moreover, Ott et al. [34] conducted a study on flame acceleration and observed how laminar flames and laminar boundary layers in narrow tubes were capable of promoting exponential increases in flame propagation velocities versus time. The role of the flow generated by the gas expansion ahead of the flame front was found to be like a piston, creating pressure / expansion waves as the flow was accelerating / decelerating.

These relatively recent works have been the onset of several investigations on flame acceleration using laminar flame approximations. As mentioned before, a key piece when exploring DDT events is the understanding of the mechanism that makes flame to accelerate beyond the laminar flame speed, hence the importance of a simplified model to explore these mechanisms. 


\subsubsection{Laminar Flame Acceleration produced by Wall Friction}

Based on the Shelkin explanation of flame acceleration, Bychkov et al. [35] developed a formulation to describe the acceleration of flames propagating in one-end open channels, ignited at the closed end, considering adiabatic nonslip walls and realistic values of thermal expansion coefficients $\Theta=\rho_{\text {fuel mixt }} / \rho_{\text {burnt matter. The work was able to predict the initially exponential }}$ state of acceleration, the flame shape and the velocity profile of the generated mixture flow. The formulation also concluded that the acceleration rate decreases with the Reynolds number related to the flame propagation. On the contrary, the flame acceleration was found to be enhanced by larger thermal expansions. Akkerman et al. [36] extended the analysis [35] to the case of cylindrical tubes, formulating analytical expressions to describe the flame dynamics. These works were validated by numerical simulations.

The fuel mixture flow velocity, generated by the expanding burning matter, were obtained as:

$$
\begin{gathered}
u_{z, \text { channel }}=(\Theta-1) U_{w} \frac{\cosh \mu-\cosh (\mu r / R)}{\cosh \mu-\mu^{-1} \sinh \mu}, \\
u_{z, c y l}=(\Theta-1) U_{w} \frac{I_{0}(\mu)-I_{0}(\mu r / R)}{I_{0}(\mu)-2 \mu^{-1} I_{1}(\mu)},
\end{gathered}
$$

for channels [35] and cylinders [36], respectively. The associated flame acceleration rates were given by

$$
\begin{aligned}
\sigma_{\text {channel }} & =\frac{(\operatorname{Re}-1)^{2}}{4 \operatorname{Re}}\left(\sqrt{1+\frac{4 \operatorname{Re} \Theta}{(\operatorname{Re}-1)^{2}}}-1\right)^{2}, \\
\sigma_{c y l} & =\frac{\operatorname{Re}}{4}\left(\sqrt{1+\frac{8(\Theta-1)}{\operatorname{Re}}}-1\right)^{2},
\end{aligned}
$$

where $U_{w} \propto \exp \left(\sigma U_{f} t / R\right), \mu=\sqrt{\sigma R e}$, with $R e=R U_{f} / v$ the Reynolds number related to the flame propagation; $I_{0}(r)$ and $I_{1}(r)$ are the modified Bessel functions of zeroth and first orders. Figure 2.6 shows a comparison of the results obtained in [35] and [36] analytically and numerically. The flow velocity profile, as given by Equation (5), is scaled by the channel width $R$ and the instantaneous maximum flow velocity $U_{\max }$ (attained at the centerline); the flame acceleration rates are presented for various $R e$. 


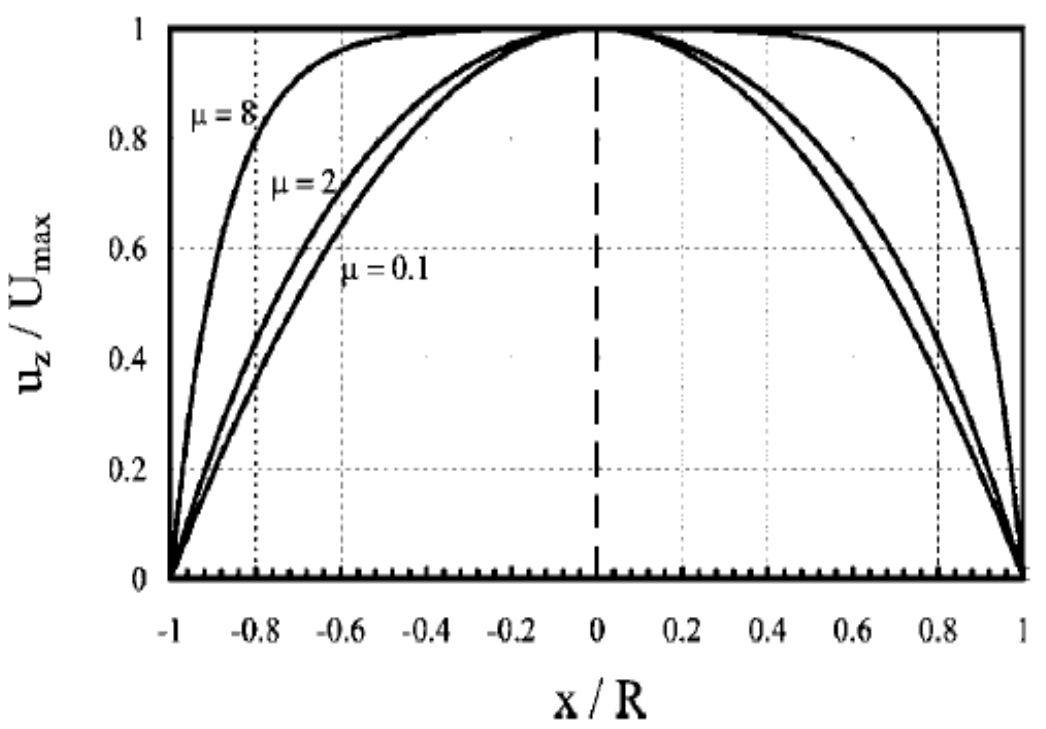

a)

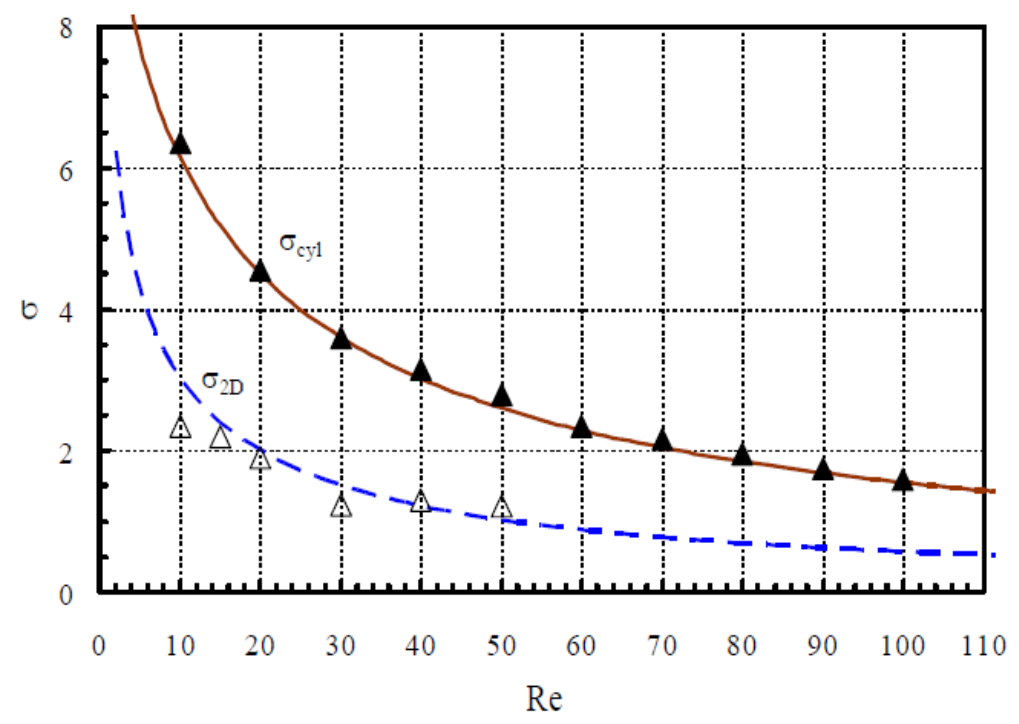

b)

Figure 2.6. a) Velocity profile of the unburned mixture, given by Equation (5) [35]. b) Acceleration rate versus the Reynolds number related to the flame propagation. Dashed and solid lines are given analytically by Eq. (7) and Eq. (8). Empty and filled markers represent the calculated acceleration rates from the simulations of channels and axisymmetric tubes, respectively $[35,36]$ 
Three stages of spontaneous flame acceleration, in two-dimensional (2D) channels, with one end open, were determined by Valiev et al. [37], starting at low flame speeds $\left(U_{f} \approx 10^{-3} c\right.$, where $c$ is the speed of the sound): first, an exponential acceleration in a quasi-isobaric regime; second, a nearly linear increase in the flame velocity; third, a saturation to a steady high-speed deflagration velocity, which could correlate with the Chapman-Jouguet deflagration velocity. The study [37] addressed the role of the gas compression ahead and behind the flame front in the establishment of these regimes.

Akkerman et al. [38] explored, numerically and analytically, the differences in the dynamics of the flame evolution attained in cylindrical tubes and 2D channels with adiabatic walls, including one and both ends open. In 2D open channels, initially planar flame fronts became concave and oscillate regularly. Concave flame fronts in open tubes turned to convex form after the first oscillation, which was followed by an exponential acceleration. The criterion for such a difference was related to the thermal expansion and combustion configurations, establishing a critical value of the thermal expansion ratio $\Theta_{c}$ that separated the accelerating from the oscillating character of the flame.

\subsection{Ultra-Fast Flame Acceleration in Obstructed Conduits}

Within the investigation of pulse detonation engines (PDE), where the control over the deflagration to detonation transition is very desirable, Roy et al. [39] proposed the inclusion of regular and irregular obstacles inside the combustors to promote DDT, obtaining detonation waves with the fuel mixture ignited by a low-energy source. Cicarelli et al. [40] performed experiments to investigate the effect of the obstacle size and spacing in early stages of flame acceleration in tubes. The authors concluded that the effect of the space between obstacles is important only when the obstacle size is large. In this case, the strongest flame acceleration was reported when the space between the obstacles was equal to the tube diameter. Also, it was noticed that the folding of the flame is the controlling mechanism of the initial flame acceleration.

Bychkov et al. [41] provided a mathematical description of the extremely strong flame acceleration observed experimentally in [39] and [40]. The formulation [41] related this acceleration, much stronger than that produced by non-slip walls, to a powerful jet-flow produced by delayed combustion in the region between obstacles ('pockets'). This investigation 
provided expressions for the exponential acceleration rate, and it was compared to the experimental results of Cicarelli et al. [40] and to numerical simulations performed in the same work [41]. The flame propagation resembled the finger flame evolution, but with the acceleration lasting much longer, i.e. without terminating with the flame skirt touching the side wall.

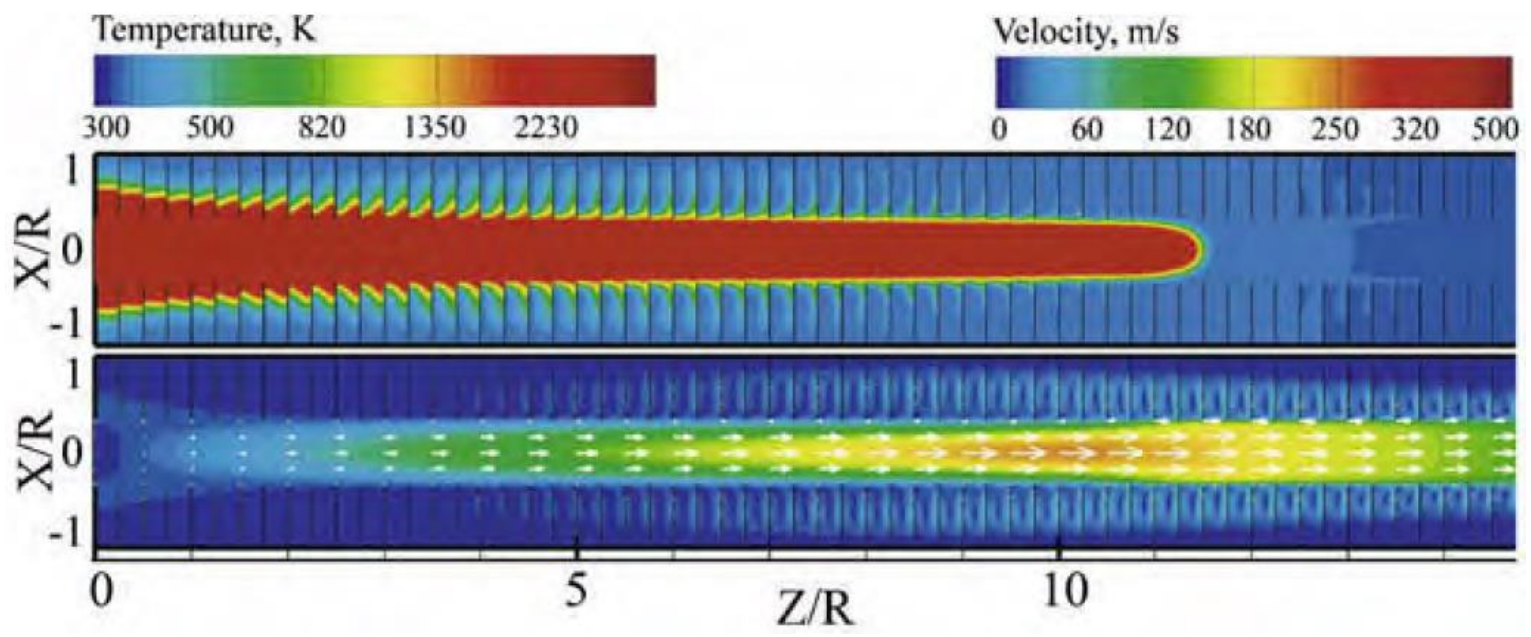

Figure 2.7. Flame propagation in obstructed channels as described by Valiev et al. [42]. Temperature (top) and Velocity (bottom) distribution, $\Theta=8, \Delta Z / R=0.25, \alpha=2 / 3$.

The work [41] was further extended by Valiev et al. [42], Figure 2.7, which also included cylindrical chambers. As in the wall friction mechanism, the flame velocity was found to saturate to a quasi-steady value, supersonic in the laboratory reference frame, which could be correlated with the Chapman-Jouguet deflagration speed. Analytical expressions for the evolution of the flame tip position $Z_{f}$ in two-dimensional channels [41] and axisymmetric cylinders [42] were given by

$$
\begin{aligned}
& \frac{Z_{f, \text { channel }}}{(1-\alpha) R}=\frac{\Theta}{\Theta-1}\left[\exp \left(\sigma U_{f} t / R\right)-1\right], \\
& \frac{Z_{f, \text { cylinder }}}{(1-\alpha) R}=\frac{\Theta}{2(\Theta-1)}\left[\exp \left(\sigma U_{f} t / R\right)-1\right] .
\end{aligned}
$$

In these expressions, the blockage ratio $\alpha$ characterizes the size of the obstacles as a fraction of the radius/half-width R. Similar to the previous sections, $\Theta$ represents the thermal expansion of the fuel mixture, $U_{f}$ the planar flame velocity and $\sigma$ the acceleration growth rate, which was found to be bigger for the axisymmetric cylindrical case than that for the planar channel [42]: 


$$
\begin{gathered}
\sigma_{\text {channel }}=\frac{\Theta-1}{1-\alpha}, \\
\sigma_{\text {cylinder }}=2 \frac{\Theta-1}{1-\alpha}\left(1+\frac{1}{2(\Theta-1)}\right) .
\end{gathered}
$$

\subsection{Flame Propagation in Non-Adiabatic Tubes and Channels}

Since the combustion process is characterized by the occurrence of multiple phenomena in a very short time, it seems reasonable to separate effects such as the momentum and energy transfer at the chamber boundaries by considering, i.e., adiabatic conditions, as undertaken in most theoretical and numerical studies on laminar flame acceleration in tubes and channels. Energy losses at the walls have been accounted primarily when studying combustion extinction, flame shape and propagation, and flame stability. However, non-adiabatic conditions have not been extensively included in the exploration of accelerating flame regimes because of the mitigating effect that heat losses to the walls produce on the combustion course. Since this investigation is dedicated to micro-tubes, it could be expected that the heat transfer process at the walls would be an important component of the analysis, strongly modifying the different mechanisms of flame acceleration related to wall conditions.

Hackert et al. [43] investigated near-suppressing conditions of laminar flames in parallel plates and cylindrical ducts. The numerical investigation focused on the shape and propagation of the flame in relation to the chamber geometry and heat loss rates at the walls. The obtained quenching criterion established a proportion between the Peclet number (relating the pipe width to the flame thickness) and the square root of the overall heat loss coefficient, with the radiation inside the channel playing a rather opposite role, inhibiting quenching.

Daou and Matalon [44] examined, analytically and numerically, the flame propagation in twodimensional channels, considering heat losses at the walls by thermal conduction and a prescribed Poiseuille flow of variable intensity. The attention was focused on the extinction conditions in relation to the chamber dimensions, specifically, two modes of combustion extinction were observed: 1) flame extinction in narrow channels (channel width less than $15 L_{f}$ ) due to excessive heat losses; and 2) partial flame extinction in wider channels. The heat losses magnitude was found to be proportional to the square of the channel width. Moreover, the 
direction of the flow also proved to be relevant. When it was directed towards the burned gas, the gas was more susceptible to the heat losses. In contrast, the flame was less sensitive when directing towards the fresh mixture.

In order to study combustion characteristics and flame stability at different operating conditions, Norton and Vlachos [45] modeled micro-burners of variable thickness and thermal conductivity. The results showed transverse gradients of temperature and reaction rate along these small widths. Isothermal wall conditions were approached when thick walls and/or larger thermal conductivities were employed. Also, conditions for flame stability as a function of the wall thermal conductivity and flow rate were identified.

Kagan et al. [46], investigated numerically the premixed flame propagation in 2D, semi-infinite channels, including isothermal wall conditions. The study extended previous investigations considering adiabatic conditions, where the hydraulic resistance was found to promote DDT [32]. When isothermal wall conditions were in place, it was noticed how the preheating of the fuel gas produced by the hydraulic resistance was mitigated as a result of the heat lost to the walls, making DDT inviable. However, the study [46] obtained conditions for DDT when the combustion kinetics was modeled as bimolecular, as a result of the higher sensitivity of the reaction to the pressure and temperature changes.

The effect of heat losses in propulsion devices were investigated by Gamezo and Oran [47]. In this work, numerical simulations were performed, considering 2D narrow channels, with one end open. Three thermal boundary conditions were used: adiabatic, isothermal and walls modified by different heat transfer coefficients, which represented diverse insulating materials. The results showed that the gas outflow velocity was reduced when the heat losses were bigger, pointing out the importance of the appropriate insulating material selection for propulsion devices operating at laminar conditions.

\subsection{Combustion Characteristics once DDT is triggered}

Once the detonation is triggered, the combustion process is significant modified, as mentioned in Section 1. In tubes, it is produced after the acceleration of the flame propagation, which determines conditions for the formation of a detonation wave that propagates at a supersonic speed [19]. 
The detonation is initiated by the adiabatic ignition of the fuel mixture ahead of the flame front, produced by the shock wave. This initiation, in turn, is closely connected to the fuel composition, wall conditions and combustion regime [19], e.g. the initiation time can vary significantly with the flame propagation direction, being sooner when the flame moves from the closed end of the tube to the open one than in the opposite direction. Detonations can be characterized by the cell size $\lambda$ [48], which is a length parameter of detonations with transverse structure, as seen in Figure 2.8.

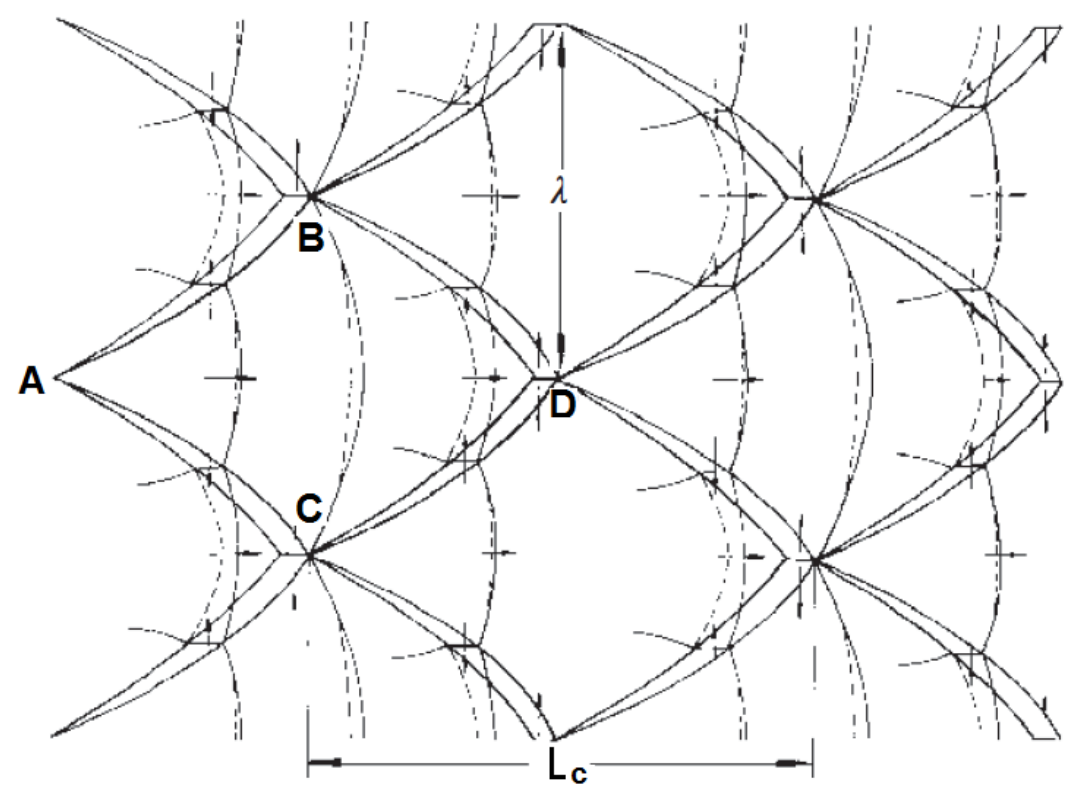

Figure 2.8. Schematic of the evolution of a cellular detonation front, as described by the trajectory of the triple points (e.g. A, B, C and D). Cell parameters cell size $\lambda$ and cell length $L_{c}$ are also shown [49] .

The cell size is typically measured by performing experiments and numerical simulations [50], and it is an indicator of the fuel composition sensitivity to the process, where smaller values are related to most detonable compositions [49]. Moreover, the possibility of a detonation initiation has been correlated to the value of the cell size, as a characteristic of the fuel mixture [51].

The cell width $\lambda$ has been also related to the detonation reaction zone width $\delta$ with the purpose of being able to obtain $\lambda$ from detailed chemical kinetic calculations. Many attempts to establish a correlation between these two parameters, $\lambda$ and $\delta$, have been done on the basis of the Zeldovich-Von Neumann-Doring (ZND) model [52], where the reaction zone width can be 
found, e.g., by considering the location of the maximum gradient of temperature behind the leading shock [52].

Although the ZND model can capture the effects of the detonation 'static' parameters such as temperature, pressure and fuel composition on the cell width $\lambda$, at least qualitatively, a correlation $\lambda / \delta$ based on this model is not reliable, because of its dependence on the fuel composition and initial conditions. This result has been attributed to the one dimensional nature of the ZND model which should deal with the multidimensional structure of real detonations [50].

In order to overcome this limitation, Gavrikov et al. [50] proposed the incorporation of stability effects into the structure of the wave, recognizing that stability parameters can determine conditions for strong and irregular transverse waves (highly unstable) or weak and regular waves (stable conditions). In this study [50], the stability effects on the wave was introduced by two parameters, namely: the dimensionless activation energy, $E_{a} / R_{p} T_{0}$, and the ratio of the mixture chemical energy to initial thermal energy $Q / C_{V} T_{0}$. As a result, a function including these two parameters was proposed to calculate the $\lambda / \delta$ ratio, showing good agreement with experimental results.

This is an example of how complex the detonation problem is, since it includes variables into the analysis that can be approached by simple models, as the Chapman-Jouguet theory which is based on the application of conservation laws across the detonations front, and variables that require a knowledge of the wave structure, hence of the chemical reaction rates [49]. One example of the latter is the critical tube diameter, which characterizes the combustion process occurring in tubes once a planar detonation wave leaves the confinement. If the tube diameter is larger than a critical value, the planar wave will become a spherical wave, otherwise it will decouple the reaction zone from the shock wave, resulting in a deflagration wave [53]. The reason for such a result relies on the rarefication of the gas as it expands outwards in the unconfined region. The expansion cools the shocked gases, increasing the induction time, leading to the decoupling of the reaction zone from the shock front [49].

This dependence of the detonation characteristics on the chamber geometry can be also observed by relating the possibility of a deflagration to detonation transition to detonation parameters. 
Peraldi et al. [54] proposed a criterion for the onset of DDT in tubes based on the cell size. By performing experiments in 18 meters long tubes with different diameters $(5,15$ and $30 \mathrm{~cm})$, including orifice ring obstacles, it was noticed that the DDT was possible when the size of the opening orifice diameter was at least that of the cell size, which corresponds to a particular fuel mixture. Moreover, this result has been extrapolated to smooth tubes, considering the orifice diameter size that of the smooth tube diameter [55]. Dorofeev et al. [56], however, proposed a different criterion for the DDT initiation. In this work [56], the authors suggested that the DDT onset is possible if $L^{\prime} / \lambda>7$, where $L^{\prime}=0.5\left(S^{\prime}+H^{\prime}\right) /\left(1-d^{\prime} / H^{\prime}\right)$, with $S^{\prime}, H^{\prime}$ and $d^{\prime}$ as seen in Figure 2.9, for obstructed tubes.

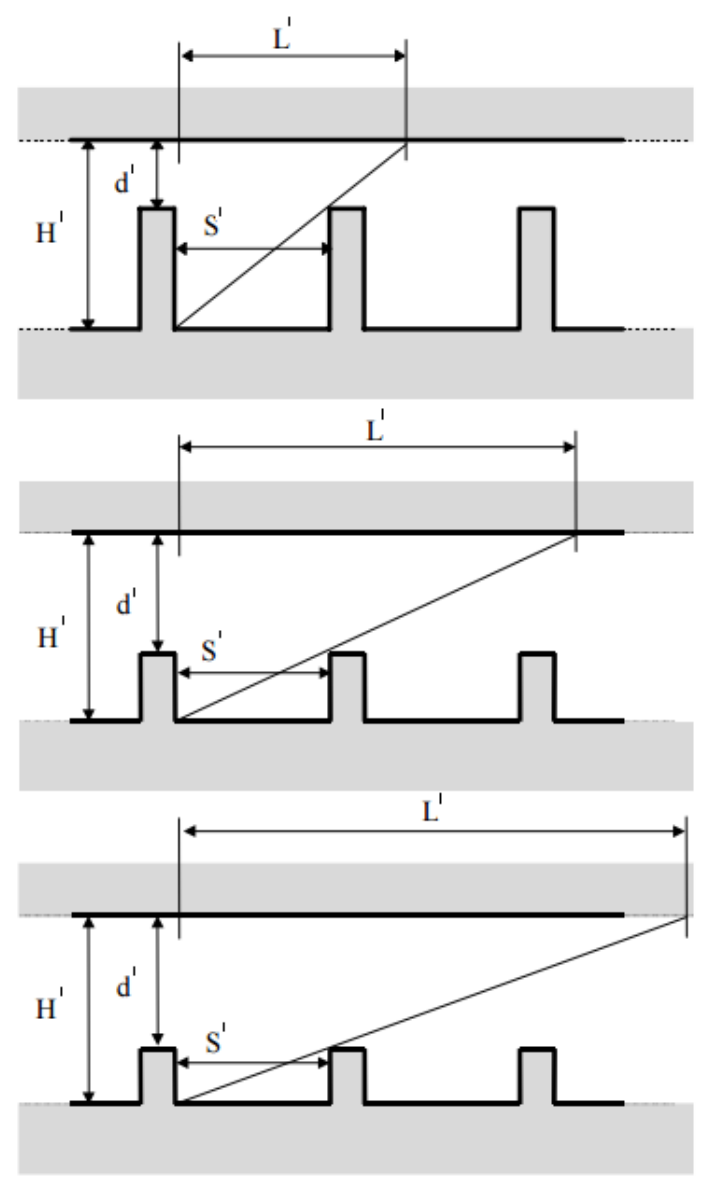

Figure 2.9. Characteristic dimensions describing the channel with obstacles investigated in [56], where the characteristic size $L^{\prime}$ is presented.

Moreover, DDT triggering by the SWACER mechanism (Shock Wave Amplification by Coherent Energy Release) [57] has been related to another length parameter, namely, that of the 
size of the 'hot spot' region (where the mixture is close to self-ignition), which should be of the order of $10 \lambda[58]$.

Another important parameter in the study of DDT in tubes, specifically, the distance that the flame propagation takes to onset the transition, has been related to detonation parameters. Kuznetsov et al. [55] have determined that the run-up distance $\left(X_{D}\right)$ is almost inversely proportional to the initial pressure. Based on experimental results, the authors proposed a relation between the run-up distance and the cell size of the form $X_{D} \approx 550 \lambda$ for a stoichiometric hydrogen-oxygen mixture in a tube with an inner diameter value of $105 \mathrm{~mm}$. However, it was pointed out that this was not a general correlation, but it would be changed if different fuel mixtures and tube sizes were considered; in other words, the run-up distance is not a criteria for detonation formation. On the contrary, it was concluded that is the boundary layer thickness of the turbulent regime, generated in the flow ahead of the flame front, which controls DDT triggering by determining the size of the pre-conditioned regions of mixture were the transition can be initiated. The turbulent scale was characterized by the turbulent pulsation $\delta_{\text {turb }}$, and the detonation onset obtained when $\delta_{\text {turb }}=10 \lambda$. 


\section{Methodology}

This investigation explores the role of the chamber boundary conditions on the flame propagation dynamics. The analysis includes the role of adiabatic and isothermal walls on the flame propagation and morphology, acting on different scenarios determined by various thermal expansion coefficients, which characterize the energy released in the combustion process. The chamber geometry is given by 2D channels and cylindrical tubes, with non-slip walls and variable width, having one end open. Moreover, a second scenario is investigated where obstacles of various sizes and spacing have been included at the walls. In this case, the attention is put on the effect of the modified wall geometry on the combustion process.

The research activity is conducted by computational means. In this chapter, a description of the equations solved numerically is presented, along with the setup of the performed numerical simulations, boundary conditions and grid generation. Additionally, the accuracy of the solver is shown by comparing the numerical results obtained in the two configurations with those obtained experimentally in chambers of similar shapes.

\subsection{Description of the Numerical Approach}

The numerical simulations are performed by an in-house program that solves the set of mass, momentum and energy conservation equations, accounting for transport processes. The set of chemical reactions is approximated by a single irreversible one-step reaction, obeying the Arrhenius law, with the burned and unburned gases set to obey ideal gas behavior.

The solver is based on a cell-centered, finite volume scheme. It is second-order accurate in time, fourth-order in space for the convective terms, and second-order in space for the diffusive terms. The solver is adapted for parallel computations. It is available in Cartesian (both two and threedimensional) and cylindrical axisymmetric versions, with a self-adaptive structured grid, and has been widely used in combustion research, e.g. [35-38].

The set of mass, momentum, energy and species equations solved by the program reads: 


$$
\begin{gathered}
\frac{\partial \rho}{\partial t}+\frac{1}{r^{\beta}} \frac{\partial}{\partial r}\left(r^{\beta} \rho u_{r}\right)+\frac{\partial}{\partial z}\left(\rho u_{z}\right)=0 \\
\frac{\partial}{\partial t}\left(\rho u_{r}\right)+\frac{1}{r^{\beta}} \frac{\partial}{\partial r}\left[r^{\beta}\left(\rho u_{r}^{2}-\zeta_{r, r}\right)\right]+\frac{\partial}{\partial z}\left(\rho u_{r} u_{z}-\zeta_{r, z}\right)+\frac{\partial P}{\partial r}+\Psi_{\beta}=0 \\
\frac{\partial}{\partial t}\left(\rho u_{z}\right)+\frac{1}{r^{\beta}} \frac{\partial}{\partial r}\left[r^{\beta}\left(\rho u_{r} u_{z}-\zeta_{r, z}\right)\right]+\frac{\partial}{\partial z}\left(\rho u_{z}^{2}-\zeta_{z, z}\right)+\frac{\partial P}{\partial z}=0 \\
\frac{\partial \varepsilon}{\partial t}+\frac{1}{r^{\beta}} \frac{\partial}{\partial r}\left[r^{\beta}\left((\varepsilon+P) u_{r}-\zeta_{r, r} u_{r}-\zeta_{r, z} u_{z}+q_{r}\right)\right]=-\frac{\partial}{\partial z}\left[(\varepsilon+P) u_{z}-\zeta_{z, z} u_{z}-\zeta_{r, z} u_{r}+q_{z}\right], \\
\frac{\partial}{\partial t}(\rho Y)+\frac{1}{r^{\gamma}} \frac{\partial}{\partial r}\left[r^{\gamma}\left(\rho u_{i} Y-\frac{\eta}{\mathrm{Sc}} \frac{\partial Y}{\partial r}\right)\right]+\frac{\partial}{\partial z}\left(\rho u_{z} Y-\frac{\eta}{\mathrm{Sc}} \frac{\partial Y}{\partial z}\right)=-\frac{\rho Y}{\tau_{R}} \exp \left(-E_{a} / R_{p} T\right)
\end{gathered}
$$

where $\beta=0$ and 1 for $2 \mathrm{D}$ and axisymmetric geometries, respectively,

$$
\varepsilon=\rho\left(Q Y+C_{V} T\right)+\frac{\rho}{2}\left(u_{z}^{2}+u_{r}^{2}\right)
$$

is the total energy per unit volume, $Y$ the mass fraction of the fuel, $Q$ the energy release from the reaction, and $C_{V}$ the heat capacity at constant volume. The energy diffusion vector $q_{i}$ is given by

$$
q_{r}=-\mu\left(\frac{C_{P}}{\operatorname{Pr}} \frac{\partial T}{\partial r}+\frac{Q}{\operatorname{Sc}} \frac{\partial Y}{\partial r}\right), \quad q_{z}=-\mu\left(\frac{C_{P}}{\operatorname{Pr}} \frac{\partial T}{\partial z}+\frac{Q}{\operatorname{Sc}} \frac{\partial Y}{\partial z}\right) .
$$

The stress tensor $\zeta_{i, j}$ takes the form

$$
\zeta_{i, j}=\eta\left(\frac{\partial u_{i}}{\partial x_{j}}+\frac{\partial u_{j}}{\partial x_{i}}-\frac{2}{3} \frac{\partial u_{k}}{\partial x_{k}} \delta_{i, j}\right)
$$

in the 2D configuration $(\beta=0)$, while in the axisymmetric geometry $(\beta=1)$ it reads

$$
\zeta_{r, r}=\frac{2 \eta}{3}\left(2 \frac{\partial u_{r}}{\partial r}-\frac{\partial u_{z}}{\partial z}-\frac{u_{r}}{r}\right), \quad \zeta_{z, z}=\frac{2 \eta}{3}\left(2 \frac{\partial u_{z}}{\partial z}-\frac{\partial u_{r}}{\partial r}-\frac{u_{r}}{r}\right), \quad \zeta_{r, z}=\eta\left(\frac{\partial u_{r}}{\partial z}+\frac{\partial u_{z}}{\partial r}\right) .
$$

Finally, the last term in Eq. (14) takes the form

$$
\psi_{\beta}=\frac{2 \eta}{3}\left(2 \frac{u_{r}}{r}-\frac{\partial u_{r}}{\partial r}-\frac{\partial u_{z}}{\partial z}\right)
$$

if $\beta=1$ (axisymmetric), and $\psi_{\beta}=0$ if $\beta=0$ (2D). Here $\eta$ is the dynamic viscosity, $\operatorname{Pr}$ and $S c$ the Prandtl and Schmidt numbers, respectively. The fuel-air mixture and burned gas are assumed to be perfect gases of constant molar mass $m=2.9 \times 10^{-2} \mathrm{Kg} / \mathrm{mol}$, with $C_{V}=5 R_{p} / 2 \mathrm{~m}, C_{P}=$ 
$7 R_{p} / 2 m$, and the equation of state $P=\rho R_{p} T / m$, where $R_{P} \approx 8.31 \mathrm{~J} /(\mathrm{mol} . \mathrm{K})$ is the universal gas constant. The one-step irreversible Arrhenius reaction is first order, with the activation energy $E_{a}$ and a frequency factor corresponding to a characteristic time $\tau_{R}$. Moreover, the solver does not account for radiation heat transfer effects.

\subsection{Numerical Simulations Set Up}

In this investigation, the computational program simulates the flame propagation occurring in two scenarios: 1) channels and tubes with nonslip walls, including either adiabatic or isothermal conditions, 2) channels with slip surfaces, including obstacles mounted on the walls, with either adiabatic or isothermal surface conditions. For this purpose, the solver includes different sets of boundary conditions, which are shown schematically in Figure 3.1 and 3.2.

\section{TOP: Nonslip, Adiabatic / Isothermal Wall}

CLOSED

Nonslip,

Adiabatic / Isothermal

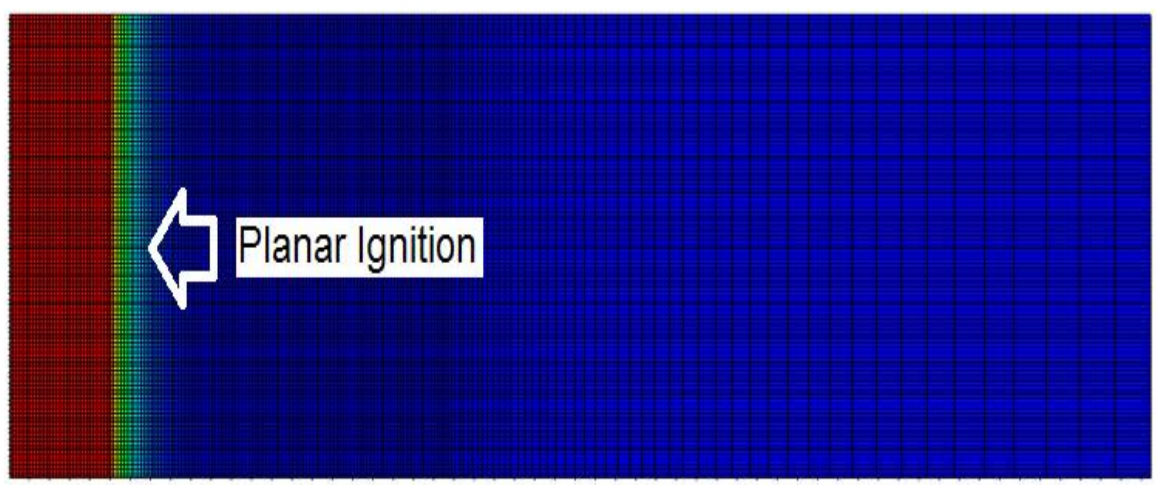

\section{OPEN}

Non Reflective Conditions

\section{BOTTOM: Nonslip, Adiabatic / Isothermal Wall IF ONLY UPPER HALF: Slip, Adiabatic Surface}

Figure 3.1.Grid domain and boundary conditions utilized for unobstructed channels.

The 'smooth' configuration, namely, channels and tubes without obstacles at the walls, is shown in Figure 3.1. A planar ignition is included to begin the process, which obeys the analytical solution given by Zeldovich and Frank-Kamenetski [3], Equation 1. The top, bottom and closed end surfaces are considered nonslip, including either adiabatic or isothermal thermal conditions (with the wall temperature varying from case to case). The open end is achieved by considering non-reflective conditions, which allows the generated flow to exit the domain, and is placed far from the flame front location. The grid is non-regular in this case, with the resolution changing in 
the horizontal direction, but constant in the vertical one. In this sense, two finer regions are generated, one interacting with the flame front, and a second one where the pressure wave generated as the flame propagates is located (not shown in Figure 3.1).

Since the gravity force is negligible in this problem, it is possible to obtain less expensive simulations by solving half-the-pipe only. In this case, slip adiabatic conditions are considered at the bottom boundary, in such a way that neither momentum nor thermal energy exchange is produced in the pipe centerline, providing a symmetry boundary condition.

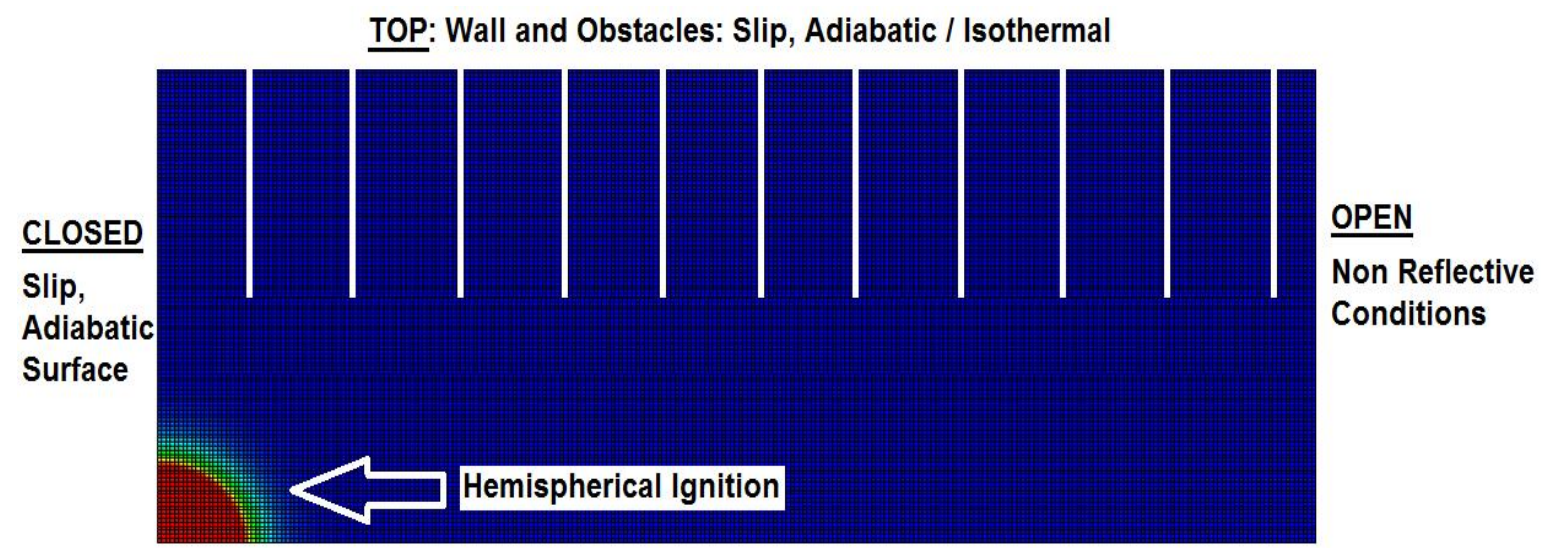

BOTTOM: Slip, Adiabatic Surface

Figure 3.2. Grid domain and boundary conditions utilized for obstructed channels.

The grid domain utilized to simulate the obstructed configuration is shown in Figure 3.2. In this case, slip conditions are prescribed at the surfaces, and a different ignition is considered, namely, a hemispherical one. The grid is regular in this case, producing more expensive simulations. For this purpose, only half of the channel has been simulated by considering a symmetry condition at the bottom. The obstacles are much thinner than that observed in the schematic, where they have been amplified for illustration purposes only.

\subsubsection{Boundary Conditions}

The different types of boundary conditions, along with the ignition form, determined the two scenarios investigated in this study. The implementation of these conditions obeys the following description:

a) Nonslip Condition: It is implemented by considering $\widehat{u}=0$. 
b) Slip Condition: This condition takes $\widehat{n} \cdot \widehat{u}=0$, where $\widehat{n}$ is the unit vector normal to the wall.

c) Adiabatic Condition: It obeys $\hat{n} \cdot \nabla T=0$.

d) Isothermal Condition: In this case, the condition is set by considering the two grid points on both sides of the surface boundary to have $T_{w}$ as average value, namely: $\left(T_{\text {in }}+T_{\text {out }}\right) / 2=T_{w}$.

e) Non-reflective Condition: This condition describes the open end of the conduits by considering $P=P_{a m b}, \rho=\rho_{f},\left(u_{x}, u_{y}\right)=0$ and $Y=1$. These values, however, are not hard imposed, being able to change in case the leading pressure wave (to be shown in Figure 3.3) reaches the open end.

\subsubsection{Self-Adaptive grid}

Horizontal variable resolution has been implemented in the solver in order to reduce the computational cost, keeping the finer resolution restricted to the flame front and to the generated pressure wave, as seen in Figure 3.3. Typical grid size values are $0.25 L_{f}$ and $0.5 L_{f}$ for the flame and leading pressure wave domains, respectively, where $L_{f}=v / U_{f} \operatorname{Pr}$ is the flame thickness. Outside the fine grid region, the mesh size is increased gradually by $5 \%$ between neighboring cells. The grid is self-adaptive, namely, it is rebuilt based on the flame front position. For this purpose, third order splines are used to interpolate the flow variables during the grid reconstruction, preserving the second order accuracy of the numerical scheme.

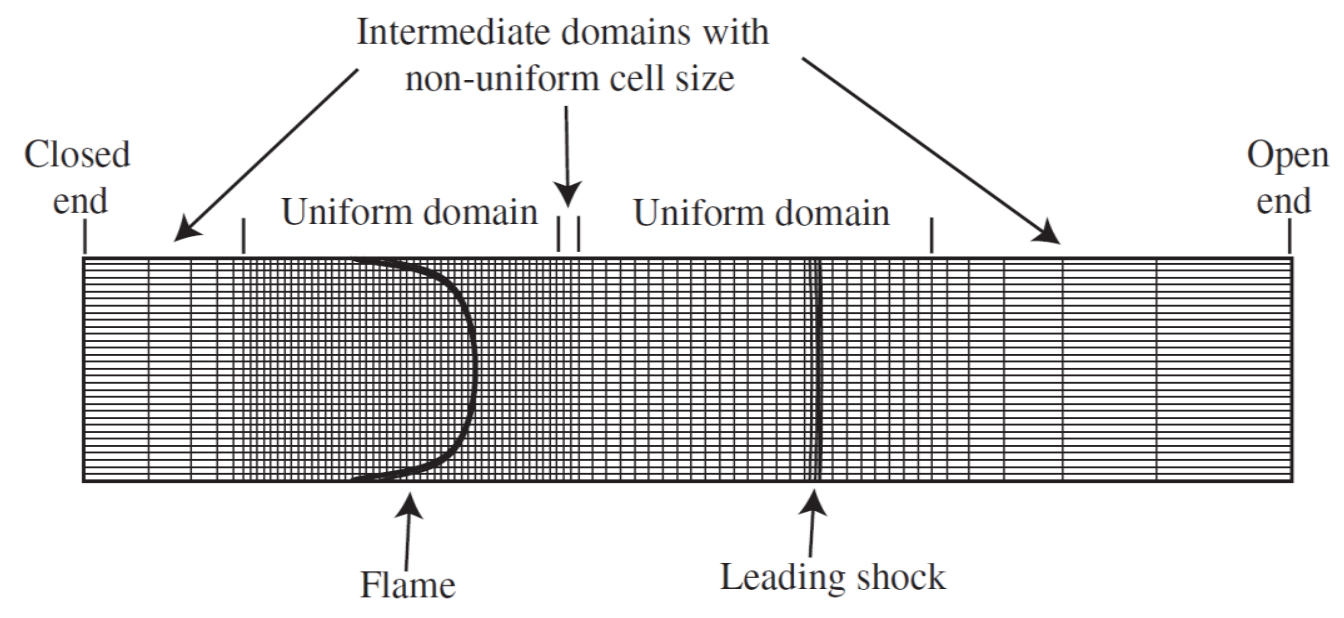

Figure 3.3. Schematic of the grid used in the numerical simulations [37]. 


\subsection{Validation}

The accuracy of the results obtained numerically have been validated by two sets of experiments; one considering a tube with 'smooth' walls, with the nonslip condition intrinsically included, and a second one where obstacles were placed at the walls of a channel. Since the pipes considered in the experiments were not subject to any particular insulation or wall material where the wall temperature could be controlled, the simulated tubes and channels were simplified to adiabatic wall conditions. The results of this comparison are presented next, pointing out the nature of the agreement and the limitations identified in the process.

\subsubsection{Nonslip Tubes}

In a recent work [59], the numerical solver has been used to model the premixed combustion process occurring in capillary tubes of radii 0.25 and $0.50 \mathrm{~mm}$. The results were compared to an experimental measurement performed in $1.5 \mathrm{~m}$. long transparent borosilicate capillary tubes, with similar radii and open at both ends [60]. The ignition was achieved in the center of the tube by applying high voltage across electrodes. The experiments considered an ethylene-oxygen fuel mixture, which was premixed using a tee before being fed into the tubes. The experimental setup is shown schematically in Figure 3.4.

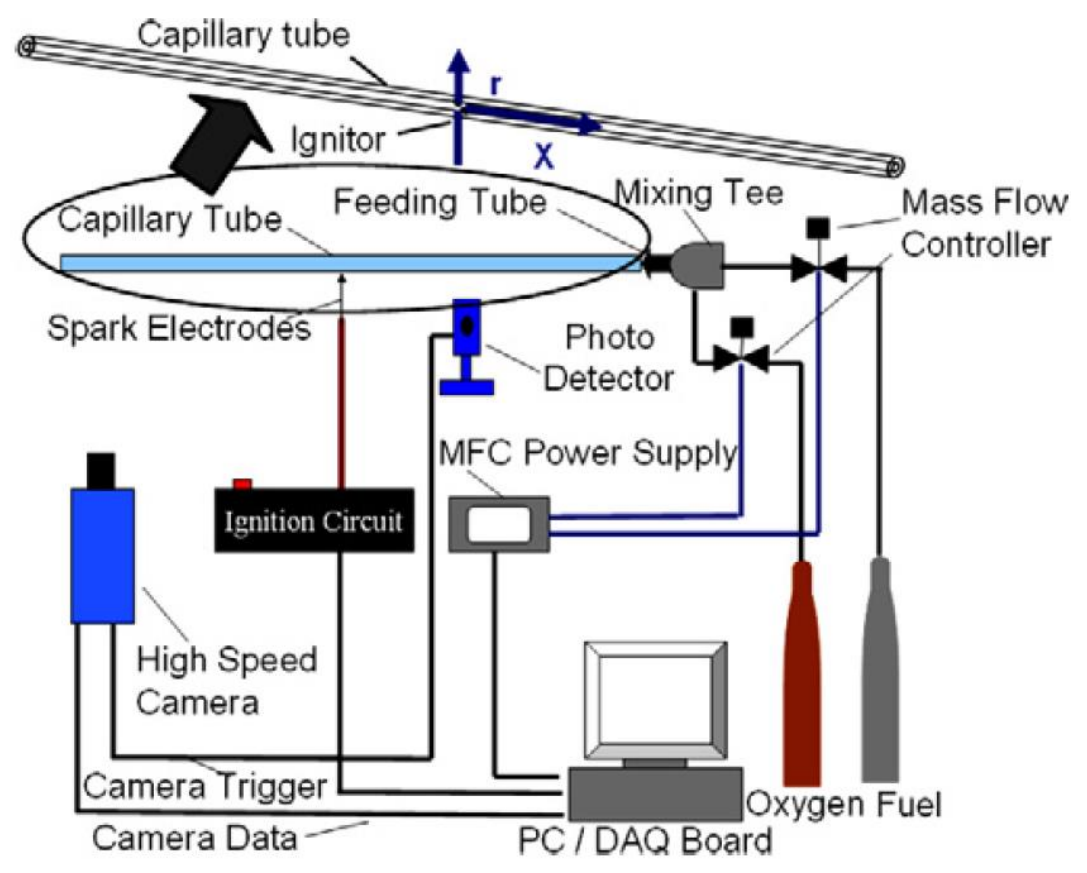

Figure 3.4. Schematic of the experimental setup [60]. 
In the numerical simulations, the stoichiometric ethylene-oxygen combustion parameters were calculated using the GasEQ software, obtaining: kinematic viscosity $v=1.72 \times 10^{-5} \mathrm{~m}^{2} / \mathrm{s}$, planar flame speed $U_{f}=5.1 \mathrm{~m} / \mathrm{s}$, adiabatic exponent $\gamma=C_{P} / C_{V}=1.34$ (i.e. the sound speed equal to $c_{0}=\sqrt{\gamma P / \rho_{0}}=331 \mathrm{~m} / \mathrm{s}$, and the initial Mach number $\left.M_{0}=U_{f} / c_{0}=0.015\right)$. The expansion factor was given by $\Theta=10.6$ and the temperature jump by $T_{b 0} / T_{0}=14$, determining the average molar mass change within the burning process to be $\widetilde{\mathrm{m}}=\Theta /\left(T_{b 0} / T_{0}\right)=1.34$.

To be able to simulate the onset of DDT, a kinematic viscosity scaling was considered in the numerical calculations, namely, the kinematic viscosity employed was $v=1.72 \times 10^{-4} \mathrm{~m}^{2} / \mathrm{s}$ instead of $v=1.72 \times 10^{-5} \mathrm{~m}^{2} / \mathrm{s}$. Since the flame thickness is proportional to the kinematic viscosity as $L_{f} \equiv v / \operatorname{Pr} U_{f}$, this change determined a larger flame thickness value, which reduced the computational vertical grid domain from $R_{t u}=0.25 \mathrm{~mm}=96 L_{f}$ and $R_{t u}=0.50 \mathrm{~mm}=$ $192 L_{f}$ to $10 L_{f}$ and $20 L_{f}$, respectively; allowing longer simulations. The scaled flame tip velocity versus the scaled time obtained experimentally and numerically is shown in Figure 3.5 and 3.6, where the initial exponential flame acceleration and posterior saturation of the combustion wave velocity, as observed experimentally at both tube diameters, have been reproduced computationally.

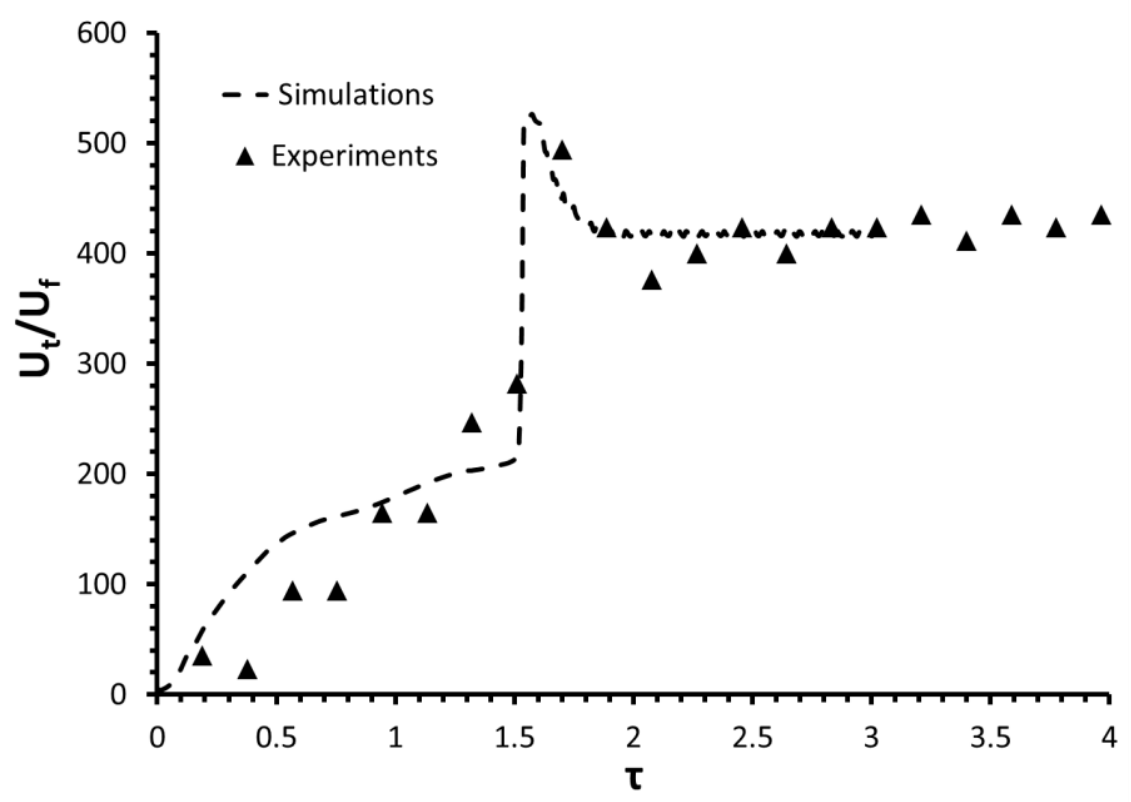

Figure 3.5. Evolution of the scaled flame tip velocity $U_{t} / U_{f}$ versus the scaled time given by $\tau=$ $t U_{f} / R_{t u}$ in a tube of radius $R_{t u}=0.25 \mathrm{~mm}$, calculated numerically and experimentally [59]. 


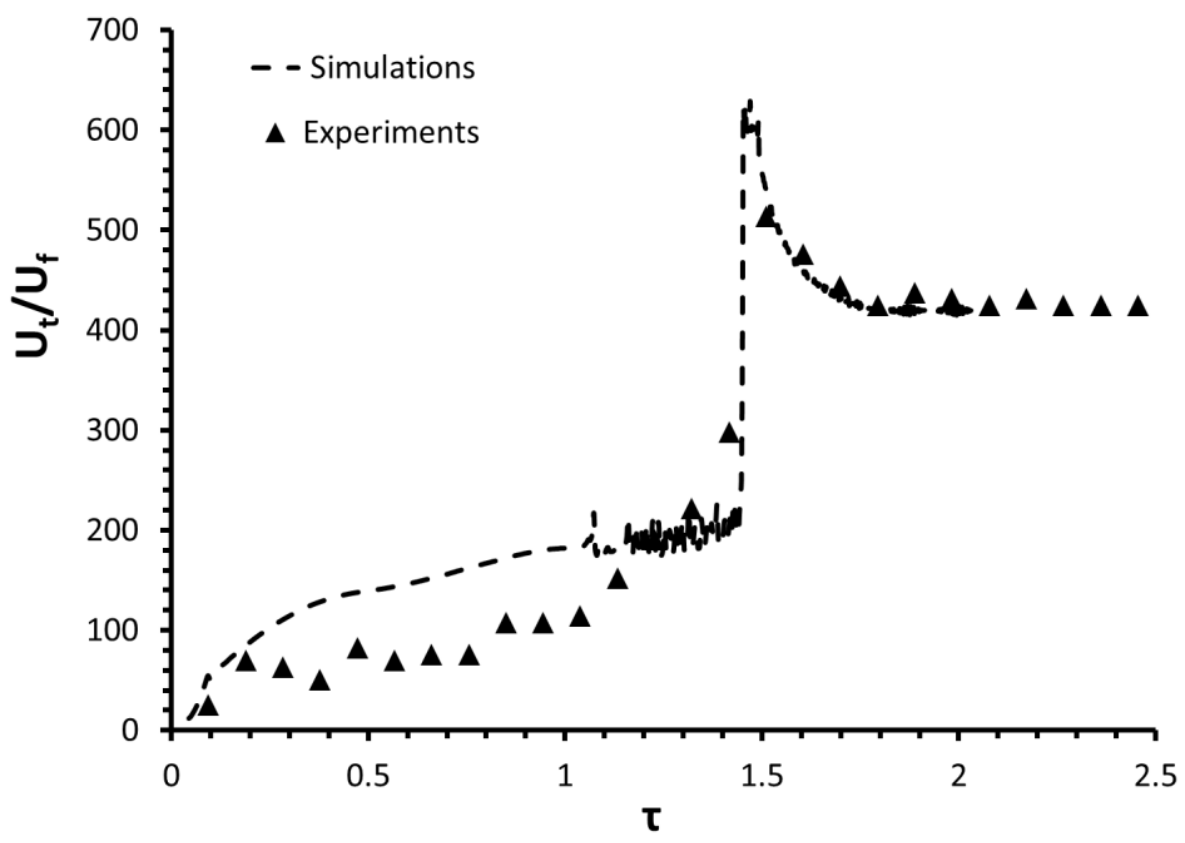

Figure 3.6. Evolution of the scaled flame tip velocity $U_{t} / U_{f}$ versus the scaled time given by $\tau=$ $t U_{f} / R_{t u}$ in a tube of radius $R_{t u}=0.50 \mathrm{~mm}$., calculated numerically and experimentally [59].

\subsubsection{Obstructed Tubes}

The validation of the obstructed configuration was done performing a qualitative comparison rather than a quantitative one. The reason laid out on the geometry dimensions implemented in the solver, characterized by extremely thin obstacles, placed in channels $40 L_{f}-60 L_{f}$ wide.

A set of experiments performed in an obstructed square cross-section channel were considered to validate this configuration [61]. The experimental setup consists on an array of obstacles mounted on the top and bottom surfaces of a channel, along the entire length, and with an equal spacing between them. The $2.44 \mathrm{~m}$ long channel used was made of aluminum 6061-T6, with a $7.6 \mathrm{~cm} \times 7.6 \mathrm{~cm}$ square cross section, and obstacles thickness equal to $1.3 \mathrm{~cm}$.

The experiments were carried out considering a stoichiometric methane-air premixed gas, with the ignition produced by an automotive spark plug at the center of one of the end flanges. Figure 3.7 shows a schematic of one of the four modules that forms the channel, including the instrumental and pressure port sites, IP and PT, and ignition locations A and B. 


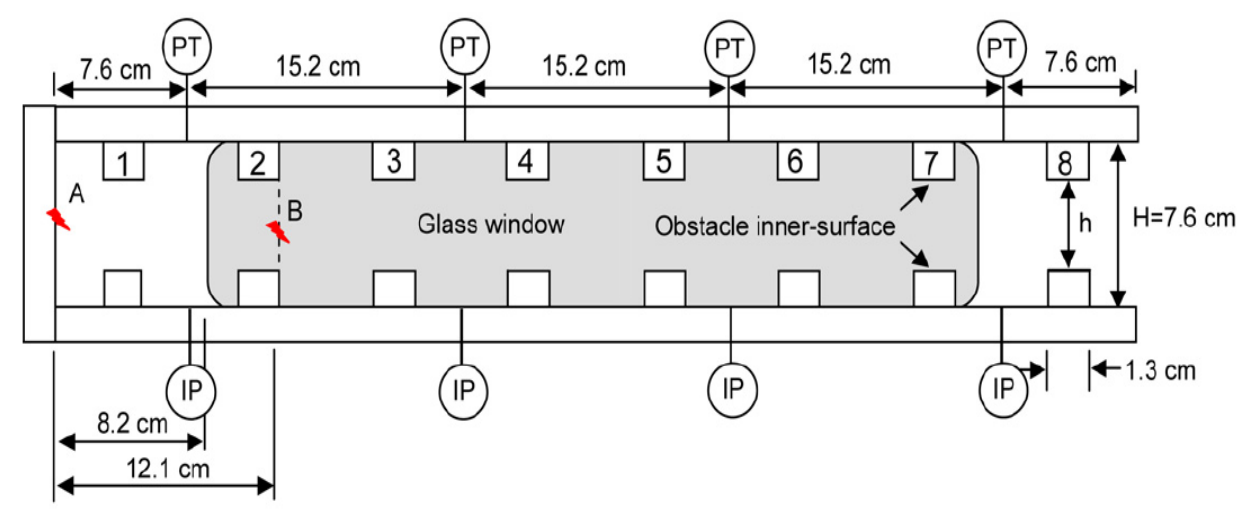

Figure 3.7. Optical module of the experimental obstructed channel [61].

In turn, the numerical simulations were performed in channels $48 L_{f}$ wide, keeping the obstacle length and spacing proportional to the experimental setup shown in Figure 3.7. Figure 3.8 and Figure 3.9 show the comparison of the flame propagation results obtained experimentally and computationally, by relating the scaled flame tip position $R_{t} / R_{c h}(1-\alpha)$ to the corresponding scaled flame tip velocities $U_{t} / U_{f}$, on the scenarios described by the obstacles spacing $\Delta Z=$ $2 R_{c h}$ and the blockage ratios $\alpha=1 / 2$ and $\alpha=1 / 3$, where $\alpha$ defines the obstacles length as a fraction of the channel width $\mathrm{l}_{\text {obstacle }}=\alpha R_{c h}$. The oscillating behavior characterized by the turbulent combustion regime is reproduced numerically; as it will discussed in Section 4.2.6.1, this regime is related to large separation between obstacles.

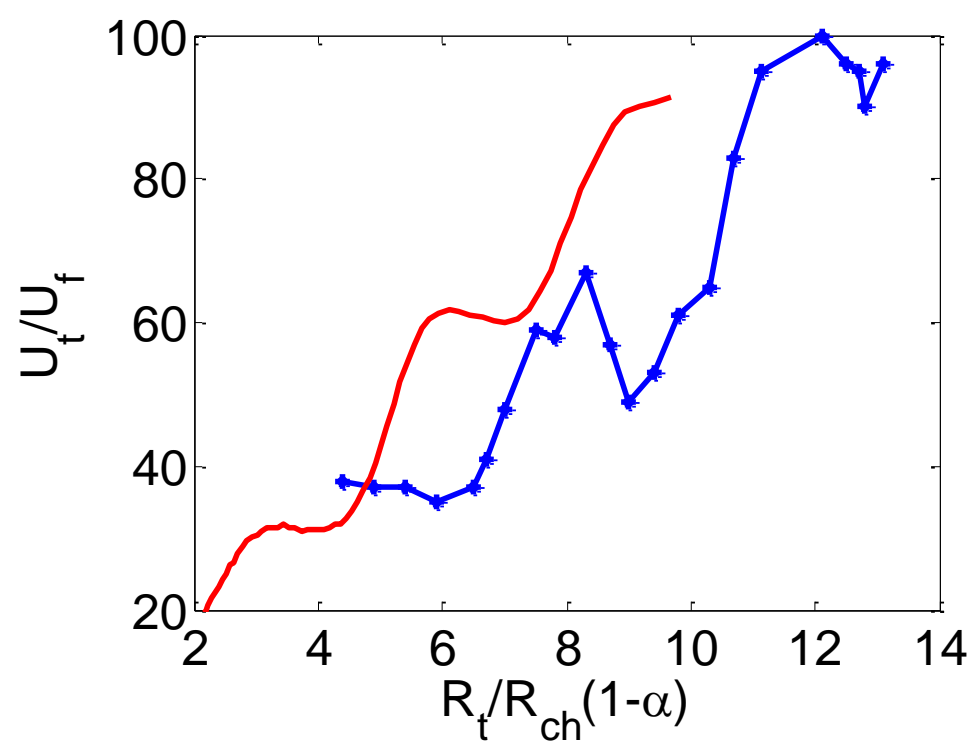

Figure 3.8. Results obtained experimentally (blue) [61] and numerically (red). Scaled flame velocity versus scaled flame tip position, $\alpha=1 / 3, \Delta Z / R_{c h}=2$. 


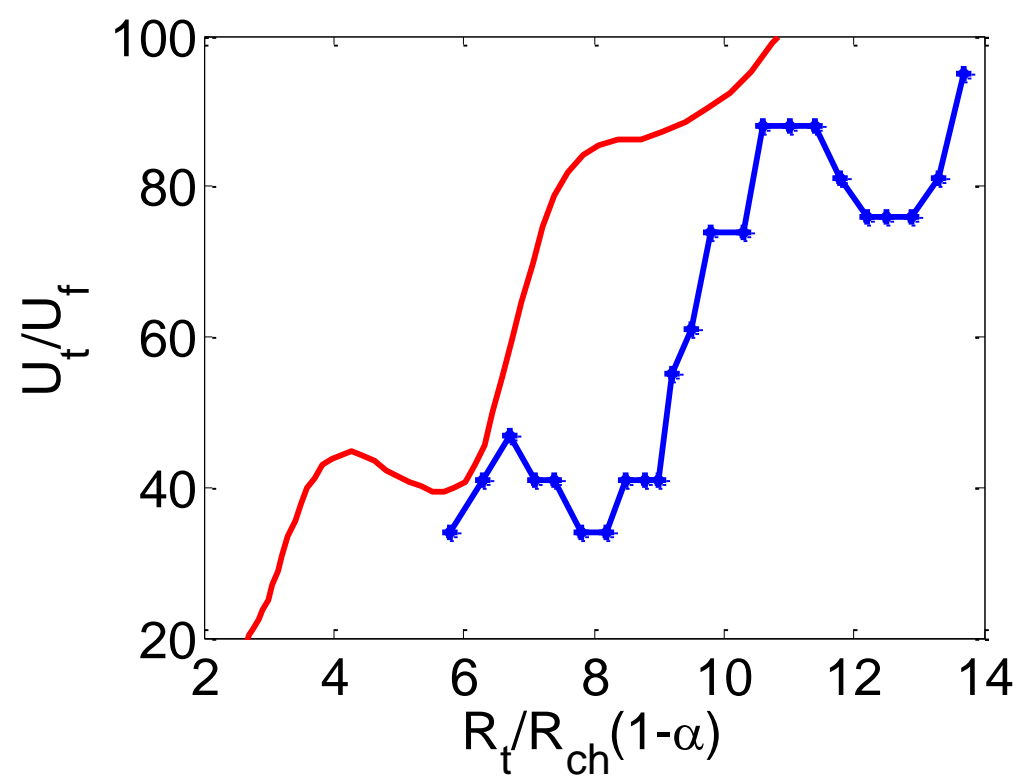

Figure 3.9. Results obtained experimentally (blue) [61] and numerically (red). Scaled flame velocity versus scaled flame tip position, $\alpha=1 / 2, \Delta Z / R_{c h}=2$.

\subsubsection{Parametric Study}

The results of the parametric study, based on the simulations of more than 150 cases, are presented in the next chapter. The values of the different parameters are listed next, although not all combinations were explored but those needed to analyze the effects of interest.

\subsubsection{Unobstructed 'smooth' configuration}

- 2 D channel half width $\left(R_{c h}\right): 5 L_{f}, 10 L_{f}, 15 L_{f}, 20 L_{f}$ and $30 L_{f}$.

- Cylindrical tube radius $\left(R_{t u}\right): 20 L_{f}$ and $30 L_{f}$.

- Thermal conditions: adiabatic and isothermal walls, with the latter given by $T_{W}=$ $300 K, 350 K, 400 K, 450 K, 500 K, 600 K, 700 K, 800 K, 900 K, 1000 K$ and $1200 K$.

- Thermal expansion coefficient $\Theta=\rho_{\text {fuel mixt }} / \rho_{\text {burnt matter }}: 5,7,9$ and 10.

The channel width varied from the value related to flame extinction in pipes $\left(5 L_{f}\right)$ to a condition where the boundary effect on the combustion process becomes less effective $\left(30 L_{f}\right)$. The wall temperatures, in turn, varied from the room temperature condition (300K) to a very high quantity $1200 \mathrm{~K}$, where spontaneous combustion have been observed, as discussed in Section 4.1.3. The thermal expansion values correspond to the range found in hydrocarbon fuels. 
The flame front thickness $L_{f}$ is defined as $L_{f}=v / U_{f} \operatorname{Pr}$ with $U_{f}$ the planar flame velocity respect to the fuel, $v$ the kinematic viscosity and $\operatorname{Pr}$ the Prandtl number. The simulations considered $\operatorname{Pr}=1, U_{f} \sim 0.35 \mathrm{~m} / \mathrm{s}$ and $v=1.7 \times 10^{-5} \mathrm{~m}^{2} / \mathrm{s}$, determining $L_{f} \approx 4 \times 10^{-5} \mathrm{~m}$. Results are presented based on the flame tip position $R_{t}$, scaled by $L_{f}$, the scaled time $\tau_{f}=$ $t U_{f} / L_{f}$, and velocity $U / U_{f}$, where $t$ is time, $U$ the flame tip velocity measured at the center of the pipe $\left(U_{c}\right)$ or near to the wall $\left(U_{w}\right)$. In certain cases, the flame front position along the channel at the center $\left(R_{c}\right)$ or near the wall $\left(R_{w}\right)$ has also been reported.

\subsubsection{Obstructed configuration}

- $2 \mathrm{D}$ channel half width $\left(R_{c h}\right): 20 L_{f}, 24 L_{f}$ and $30 L_{f}$.

- Thermal conditions: adiabatic walls and isothermal walls with $T_{W}=300 \mathrm{~K}, 600 \mathrm{~K}$ and $1000 \mathrm{~K}$. Thermal expansion coefficient: $\Theta=8$.

- Blockage ratio, which defines the obstacle height as a fraction of the channel width $\left(l_{\text {obstacle }}=\alpha R_{c h}\right): \alpha=1 / 40,1 / 20,1 / 15,1 / 9,1 / 5,1 / 4,1 / 3,1 / 2,2 / 3$.

- Obstacles spacing $(\Delta Z)$, scaled by $R_{c h}: \Delta Z / R_{c h}=0.2,0.3,0.38,0.6$ and 1 .

In this configuration, the parametric study has been focused on the geometrical variables, described by the channel width and obstacle size and spacing. According to the results in Section 4.2 , this priority correlates well with the role that geometry plays on the flame dynamics, much significant than the wall thermal conditions.

The flame front thickness $L_{f}$ is defined as in Section 3.3.3.1, $L_{f}=v / U_{f} P r$, with $U_{f}, v$ and $P r$ having the same values, determining $L_{f} \approx 4 \times 10^{-5} \mathrm{~m}$. The results are presented based on the flame tip position $R_{t}$, scaled by the channel half-width as $R_{t} / R_{c h}$, the scaled time $\tau_{f}=t U_{f} / R_{c h}$ and velocity $U_{t} / U_{f}$, where $t$ is time and $U_{t}$ the flame tip velocity.

In the snapshots corresponding to the obstructed cases (Section 4.2), white obstacles have been added to represent the obstacles position along the channel, since the original ones in the computational domain are extremely thin, and cannot be visually observed. This has been done in all the obstructed configuration snapshots, with exceptions of those including streamlines, where the obstacles and pockets are clearly noticed. 


\section{Results}

\subsection{Flame Dynamics in 'Smooth' Channels with Isothermal Walls}

In the combustor dimensions considered in this investigation, the dynamics of a propagating flame is strongly related to the conditions at the boundaries. The boundary layer thickness produced by the nonslip condition at the walls is comparable to the conduit width, therefore the associated stretching that it produces on the flame front is significant. Besides, the thermal energy exchanged at non-adiabatic walls can reach the inner part of the flame structure more effectively.

In this sense, the analysis can be performed in relation to two major sources of energy exchange at the walls: momentum exchange and thermal energy exchange. Considering a channel or tube filled with a flammable gas, with one end closed and the other open, a planar ignition at the closed end will generate a flame propagation toward the open one consuming the available fuel mixture. The flame propagates basically as a result of two mechanisms: first, the flame front moves toward the fuel mixture consuming it. This motion, in the case of a planar flame front, results in a flame propagation at a constant speed with respect to the fuel $(\sim 0.3 \mathrm{~m} / \mathrm{s}$ for the conditions considered in this investigation). Second, behind the flame, the burned gas becomes much hotter than the fresh gas mixture (e.g. 5 to 10 times higher), which decreases its density in a similar proportion, provided the isobaric and ideal gas approximations. The density reduction produces an expansion of the burned matter, which pushes the flame-gas system, in order to obtain the needed room. This second mechanism, related to the thermal expansion, is a property of the fuel, and will be extensively analyzed in subsequent sections.

As the flame propagates, it moves as a semi-transparent piston pushing the gas ahead of the flame front, generating the flow of the unburned gas. The wall friction distorts the uniform propagation of the gas flow, producing a velocity profile on it that resembles the classical Poiseuille flow, Figure 4.1. The distortion on the gas flow modifies the planar flame propagation, as the flame front acquires a similar shape. This elongates the flame front, intensifying the 
combustion process since the overall flame front surface area is increased. As a result, the flame propagates faster, pushing the gas ahead harder, further distorting its velocity profile, which elongates the flame front even more, producing a positive flame-flow feedback.

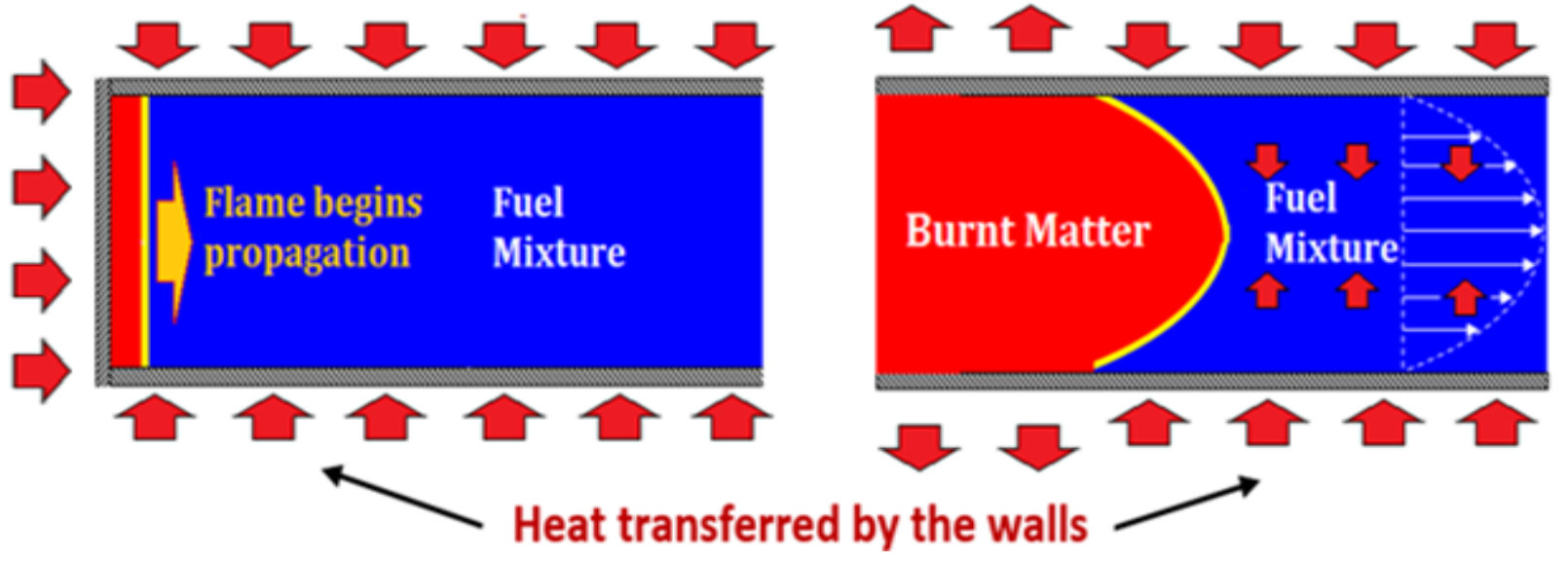

Figure 4.1. Physical description of the problem.

When the pipe walls are held at a constant temperature, heat is exchanged between the walls and the gas in two directions: toward the gas in the region ahead of the flame front (given that the gas mixture considered in the study is initially at the room temperature $300 \mathrm{~K}$, and no walls colder than that have been considered) and away of the burned gas in the region behind the flame front, since the channel/tube walls were kept at temperatures below the expected adiabatic flame temperature. This heat exchange between the walls and the unburned/burned gas modifies the gas density and consequently the thermal expansion coefficient, which plays an important role in the flame propagation intensification as mentioned before.

But further understanding of how this thermal energy exchange can modify the flame propagation dynamics is obtained by looking at the flame front-isothermal walls interaction in more detail, e.g. by comparing the effect produced by the walls when subject to different temperatures.

\subsubsection{Wall Temperature Effect on the Flame Propagation}

When the walls of a pipe where a flame propagation is taking place are kept at a constant temperature, besides the overall heat gain/loss to the gas, an intriguing modification of the flame front shape is found, as seen in Figure 4.2: 

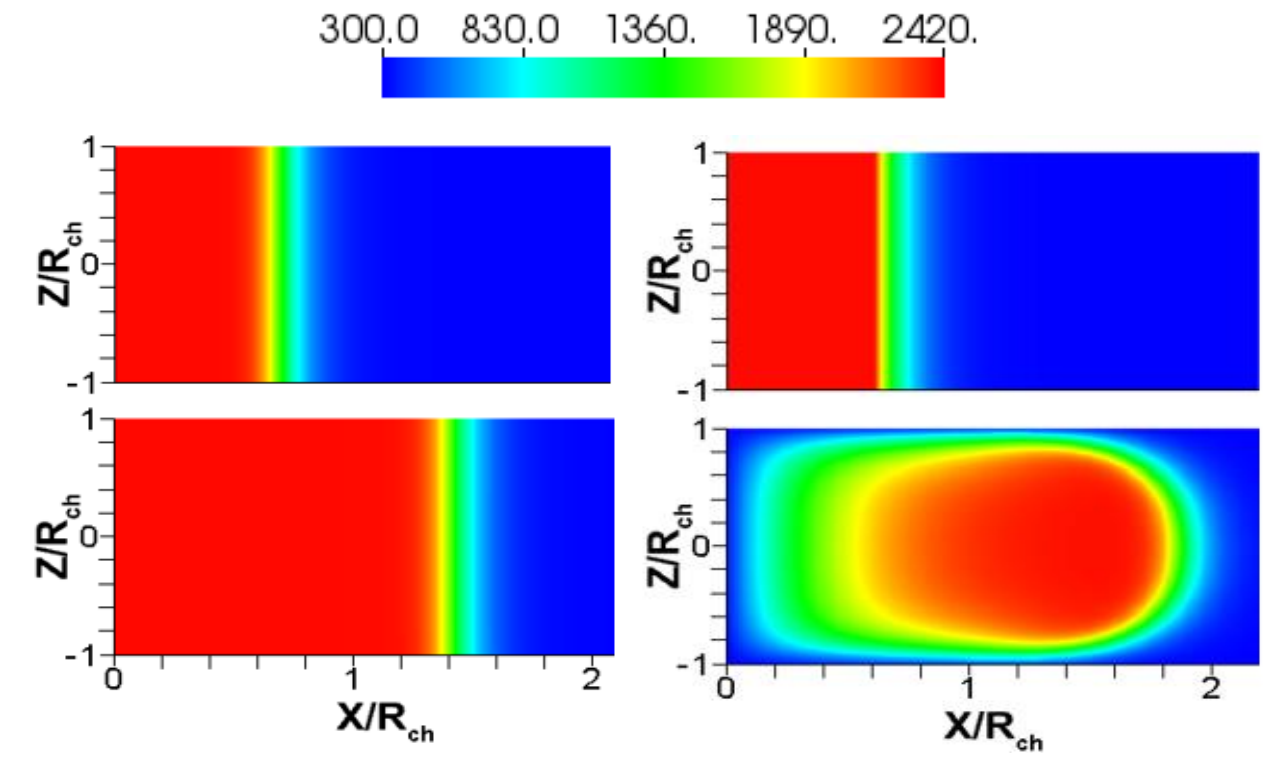

Figure 4.2. Flame propagation in adiabatic (left) and isothermal $\mathrm{T}_{W}=300 \mathrm{~K}$ (right) slip channels, $\mathrm{R}_{c h}=10 L_{f}$.

The two flame propagation scenarios, shown in Figure 4.2, have two features in common: (i) they were initiated by a planar ignition and (ii) the propagation occurs in a channel with slip walls. Only one difference has been set in their boundary conditions: on the left, adiabatic wall conditions have been employed, whereas isothermal walls were included on the right. As expected, the flame propagation in the adiabatic, slip case occurs without any significant change, say, there is not distortion of the generated gas flow (therefore no Poiseuille profile-like ahead of the flame), and consequently no flame front stretching. The flame propagation is controlled by the expanding burning matter plus the constant-rate fuel consumption at the flame front. However, when isothermal conditions are in place (Figure 4.2, right), a flame stretching is observed. There is no friction at the walls that would explain this flame front corrugation, and for the given channel width, no Darrius-Landau instability would corrugate the flame front either. Only a temperature gradient that extends from the flame front to the whole burned matter (in different levels, depending on how far the gas parcel is from the walls), has been added to the system. Certainly, a flame front distortion in the absence of nonslip walls was not expected, and it may determine a faster flame propagation in the isothermal channels as compared to the adiabatic case, if the thermal expansion coefficients were the same. But, would isothermal pipes generate larger flame stretching than adiabatic ones in nonslip channels? Moreover, would the flame then propagate faster at isothermal nonslip wall conditions as compared to the adiabatic 
ones? It is noticed that the thermal expansion ratio is reduced as a result of the heat exchanged between the gas and the walls, but, would this reduction overcome a larger flame front surface area produced by isothermal walls?

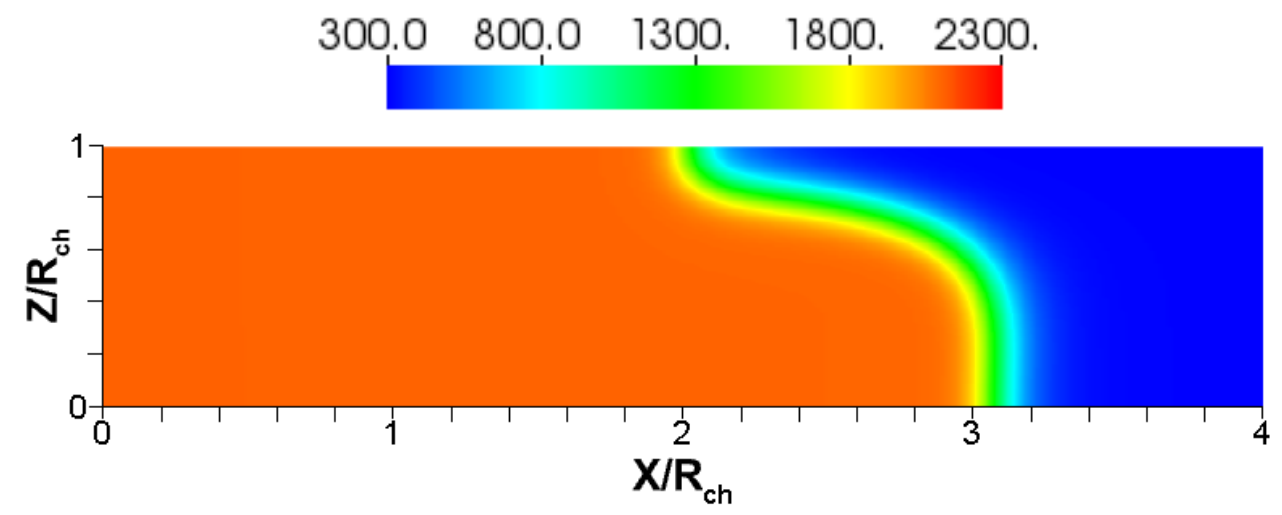

a)

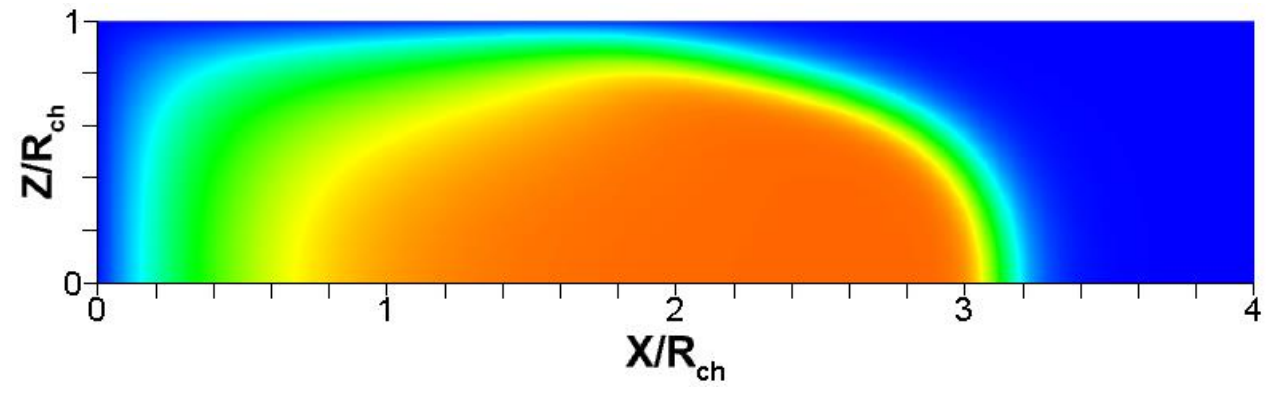

b)

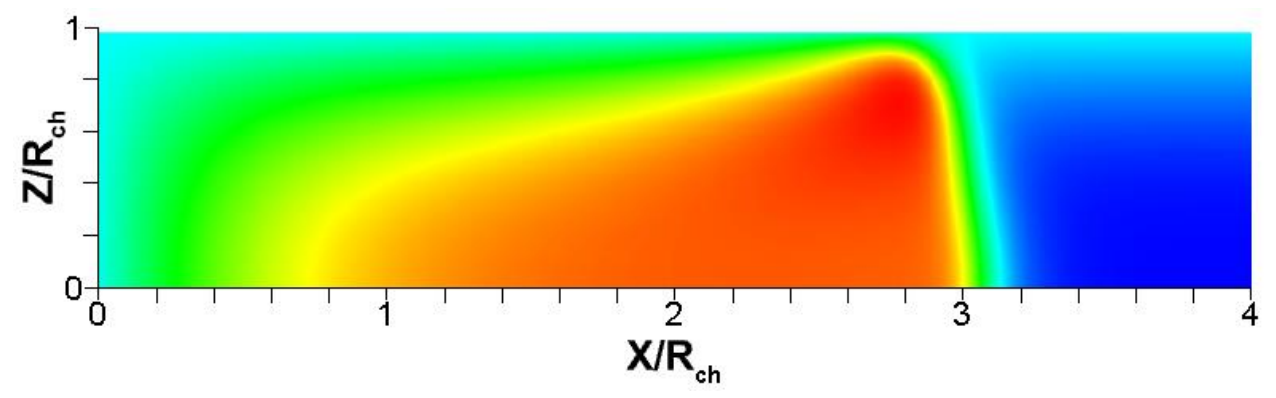

c)

Figure 4.3. Temperature distribution of the flame propagation in channels (upper half shown), $\mathrm{R}_{c h}=10 L_{f}, \Theta=7$. a) adiabatic, b) isothermal, $\mathrm{T}_{W}=300 \mathrm{~K}$ and c) isothermal, $\mathrm{T}_{W}=800 \mathrm{~K}$.

Some of these questions can be explored by considering a variety of pipe wall temperatures. Figure 4.3 shows temperature snapshots of the upper half of the channels, considering nonslip conditions this time. Specifically, Figure 4.3a presents the adiabatic case, characterized by the 
constant temperature observed in the regions away from the flame front, either ahead or behind it. In contrast, the isothermal wall conditions, considered next with fixed wall temperatures $300 \mathrm{~K}$ in Figure $4.3 \mathrm{~b}$ and $800 \mathrm{~K}$ in Figure 4.3c, produce a temperature gradient in the regions away from the flame front. Besides the difference in the burned/unburned gas temperature distributions, the flame front shapes are also different. Figure 4.4 shows the temperature contours for the cases described in Figures 4.3a and 4.3b, at two different instants each. In the adiabatic case (Figures 4.3a and 4.4a), the zero heat exchange between the gases and the walls can be identified by the angle that the temperature contours form with the walls, quasi orthogonal.
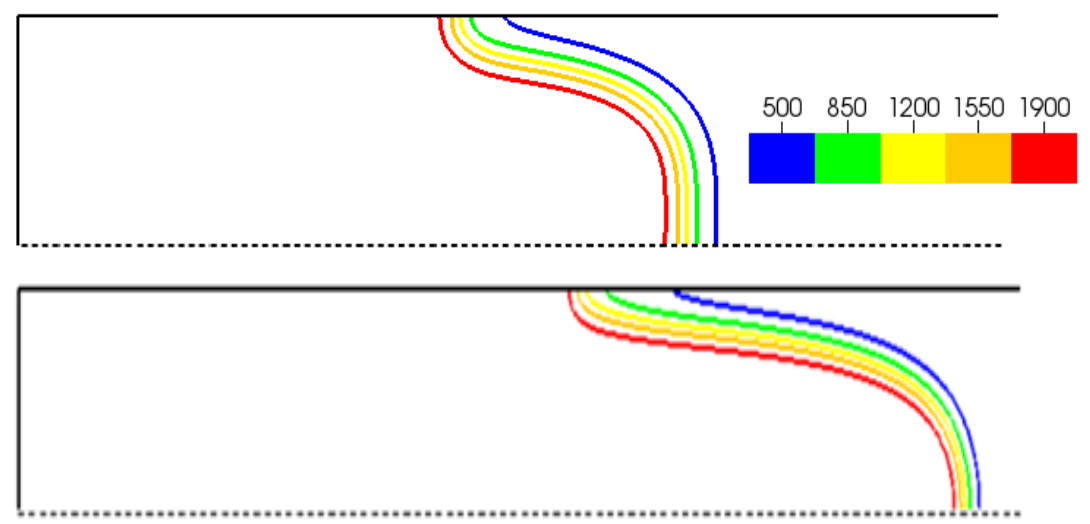

a)
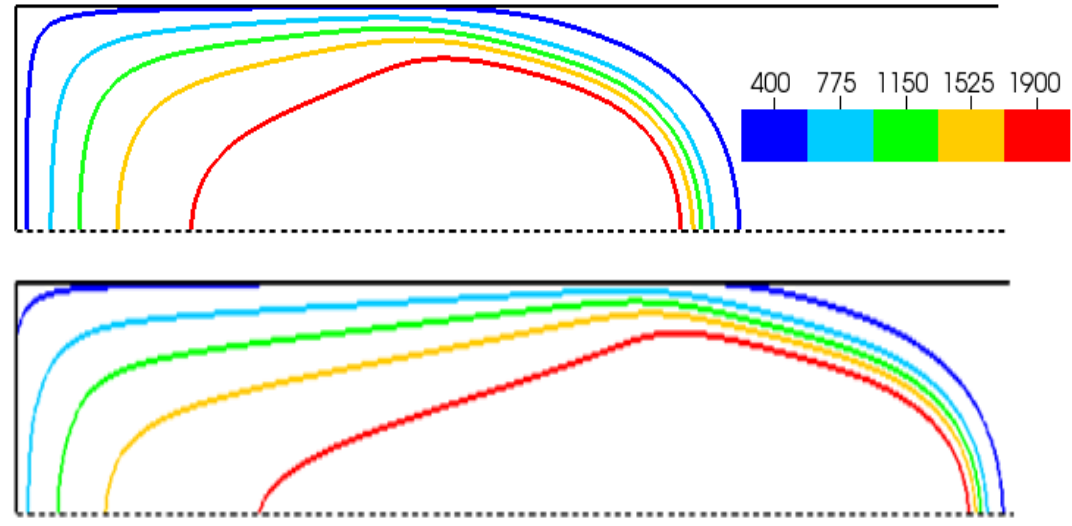

b)

Figure 4.4. Temperature contours of the flame propagation in channels (upper half shown) with $\mathrm{R}_{c h}=10 L_{f}, \Theta=7$, and different wall conditions a) adiabatic, b) isothermal $\mathrm{T}_{W}=300 \mathrm{~K}$.

Isothermal wall conditions produce a different temperature gradient since the walls force the nearby gas to be at the given wall temperature. For instance, when the wall temperature is kept at $300 \mathrm{~K}$, Figures $4.3 \mathrm{~b}$ and $4.4 \mathrm{~b}$, the colder contour is extended to the back side of the channel, 
where the closed pipe end is also kept at $300 \mathrm{~K}$. By looking at the burned gas regions in the adiabatic and isothermal wall scenarios (Figures $4.4 \mathrm{a}$ and $4.4 \mathrm{~b}$ ), it can be noticed that the cooling experienced by the burned gas in the isothermal case is so intense that the hot burned mass (i.e. identified by the $1900 \mathrm{~K}$ isotherm, red line) is significantly less than that of the adiabatic case $(\sim 50 \%)$. In addition, the gas ahead of the flame front in the isothermal case does not experience any significant temperature change, producing all together a lower volumetric expansion in the isothermal case than the obtained in adiabatic conditions.

Moreover, a stretching of the flame front, comparable to the adiabatic case, is produced by 'cold' isothermal walls ( $T_{w}=300 \mathrm{~K}$, Figures $4.3 \mathrm{~b}$ and $4.4 \mathrm{~b}$ ), which promotes the flame propagation. However, the adiabatic condition at the wall produced an "anchoring" effect on the flame front propagation, which keeps the flame front surface distorting (Figure 4.4a). The flame front elongation produced in the isothermal case increases the flame surface area, but to a steady shape, which is kept more or less constant thereafter (Figures 4.4b). In conclusion, a cold wall condition (e.g. 300K) is not expected to produce the strong acceleration observed in adiabatic conditions, although a distortion of the flame is initially produced.

Further understanding of the flame distortion resulted from the isothermal condition can be obtained if a more severe case is considered, namely, if the channel wall is kept at $800 \mathrm{~K}$ (Figures 4.3c and 4.5). In this case, the flame front elongation is less pronounced than in cold walls, but it departs from the planar flame shape anyway. The more interesting characteristic of the flame in this case is the stretching of the inner contours toward the wall, defining the shape that the flame front adopts. The thermal expansion ratio is also modified not only by the postcooling of the burned matter (which is less intense, since it is exposed to a warmer wall), but also by the preheating of the fresh fuel mixture in the vicinity of the walls, determining also a reduced volumetric expansion as compared to the adiabatic case.

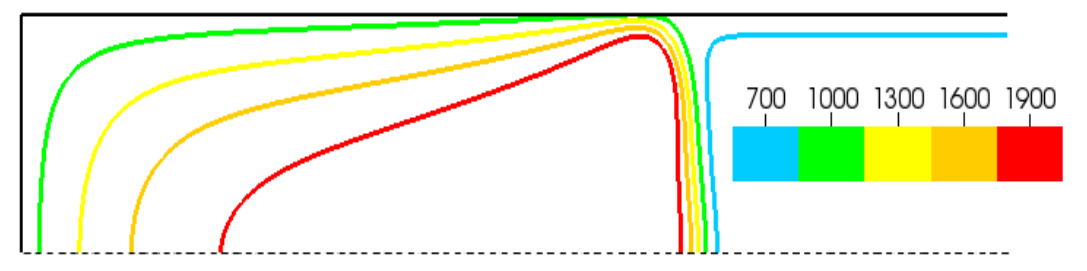

Figure 4.5. Temperature contours in isothermal channels (upper half shown), $\mathrm{T}_{W}=800 \mathrm{~K}$, with $\Theta=7, \mathrm{R}_{c h}=10 L_{f}$. 
In addition, the flame propagation related to these thermal boundary conditions can be correlated to the flow motion in the vicinity of the flame front. Figure 4.6a shows the streamlines generated in the adiabatic case. The nonslip condition works as a flow source, with the flow going toward the central part of the burned gas region, since there is no temperature gradient therein. The flow is deviated as a result of the expansion of the burning matter, which is adapting to its larger volume. When the walls remain at a constant temperature (Figure 4.6b), the flow goes toward a different location of the burned gas, since the density on the region is not uniform, looking for the less dense region. This deviation changes the momentum transferred to the flame front, which can be related to the streamline curvature behind the flame.

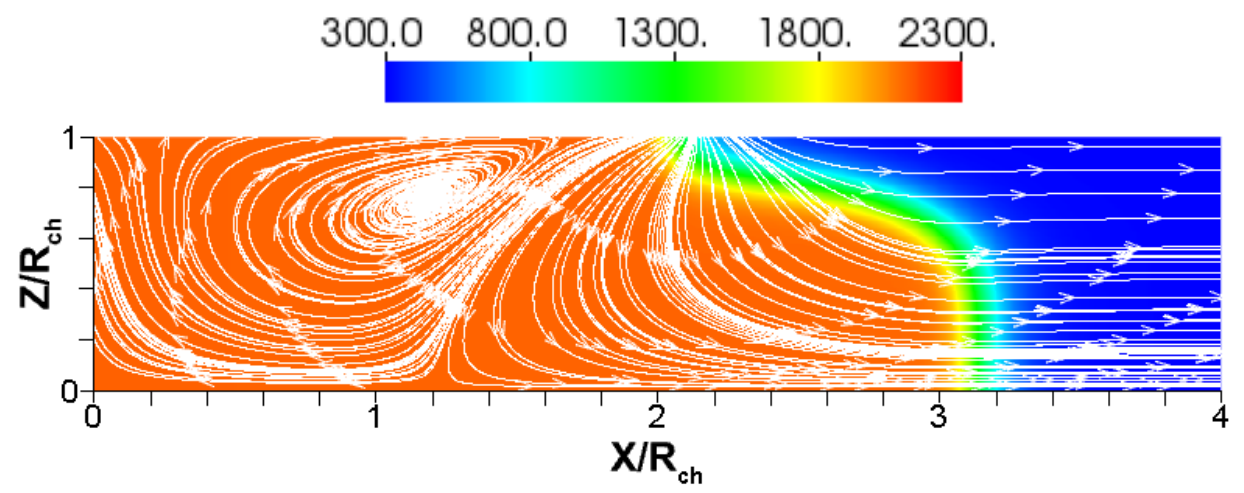

a)

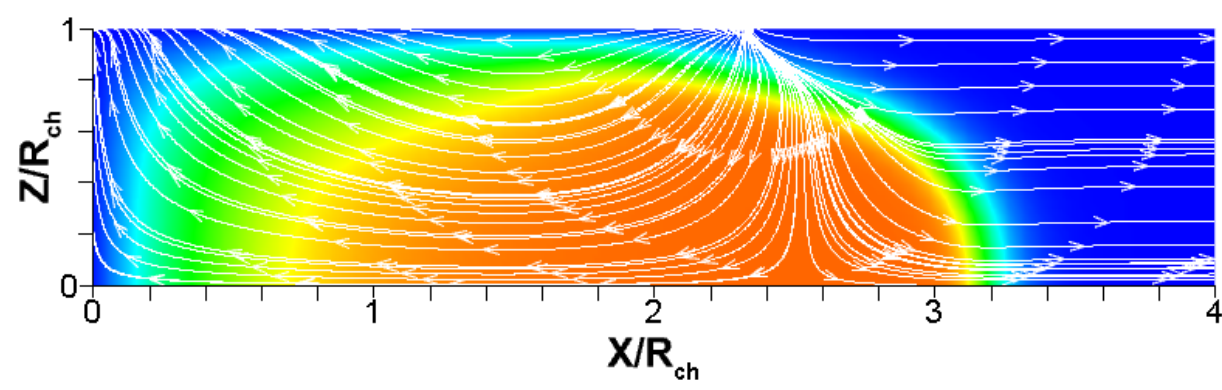

b)

Figure 4.6. Temperature distribution and streamlines of the flame propagation in channels (upper half shown), $\mathrm{R}_{c h}=10 L_{f}, \Theta=7$, a) adiabatic and b) isothermal $\mathrm{T}_{W}=300 \mathrm{~K}$.

The flame front corrugation observed at adiabatic and isothermal conditions has been explained by looking at different wall thermal conditions, and a general idea of the corresponding flame propagation velocities can be realized after this analysis: a faster flame propagation is produced under adiabatic conditions than in isothermal conditions, which may turn even slower as the wall temperature is increased, due to the less stretched flame front generated. Meanwhile, a set of new 
questions can be formulated. For example, the flame propagation observed in adiabatic pipes has been shown to accelerate exponentially, which can trigger a transition to detonation $[35,36]$. What is the nature of the flame propagation in isothermal conditions? Probably it is not exponential anymore, but what is it? Also, if a lower flame velocity is obtained by increasing the isothermal wall temperature, does this tendency stand no matter how much the wall temperature is raised? In the next section these questions are explored by comparing the flame propagation velocity attained at the different thermal conditions discussed, putting special attention to the nature of the velocity trends versus time.

\subsubsection{Flame Velocity in Isothermal Conditions}

As shown in the previous section, it is possible to modify the flame morphology and, consequently, its propagation velocity by keeping the walls at a certain temperature. Figure 4.7 compares the flame velocity when adiabatic and multiple wall temperatures are in place.

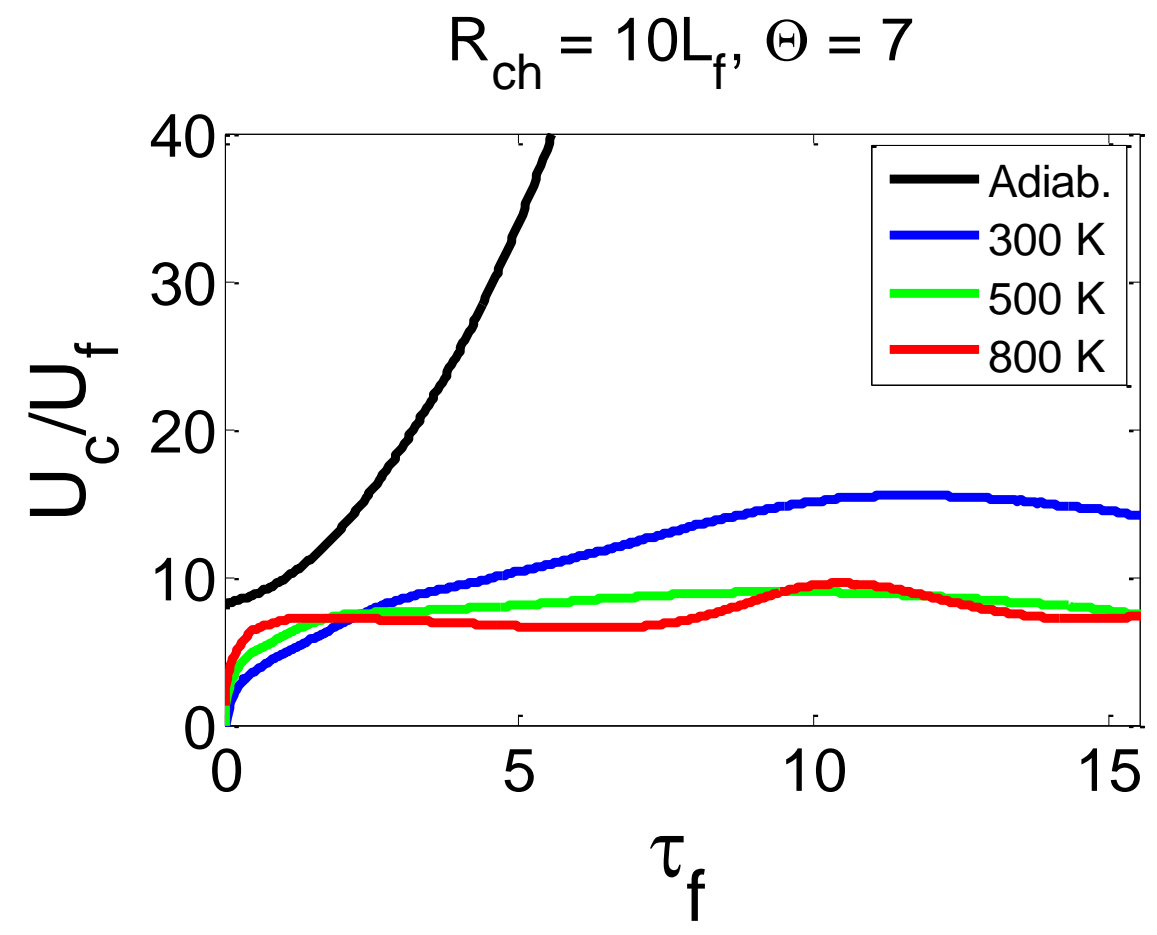

Figure 4.7. Flame velocity evolution at different wall conditions.

In this comparison, three main characteristics are observed. First, the adiabatic case is related to a much faster flame propagation as compared to the isothermal wall conditions. Second, after a short period, colder walls provide faster propagation than hotter ones. Third, the flame velocity in the hottest wall considered in Figure $4.7(800 \mathrm{~K})$ shows an oscillating behavior at later time. 
An exponential velocity of the flame propagation is observed in adiabatic conditions, which is much faster than the attained in isothermal scenarios. The flame propagation in isothermal conditions is initially intense, as seen by the curve slopes, for all the three wall temperatures considered. However, these initial large flame velocities are rapidly replaced by more moderate propagation speeds, associated with a more steady shape of the flame front. The propagation is initially faster in the hotter channels (around $0<\tau_{f}<2.5$, with $\tau_{f}=t U_{f} / L_{f}$ ), as a result of the burning enhancement attained in warmer conditions, which is later overcome by the smaller surface area of the flame front associated with higher wall temperatures. Faster flame velocities are then obtained when colder wall conditions are in place, exhibiting a linear acceleration after the tendency shift observed around $\tau_{f} \approx 2.5$. This linear increase lasts until a major cooling of the burned matter is produced, given that the wall temperature does not change in time, resulting in the deceleration of the propagation, as noticed at $\tau_{f} \approx 12$ for the $T_{w}=300 \mathrm{~K}$ case (blue line).

At the stage where a linear acceleration is attained, a weaker flame propagation is noticed as the walls get warmer. Figure 4.8 presents a more detailed description of this flame velocity decay with higher wall temperatures, by considering isothermal channel scenarios varying from $T_{w}=$ $300 \mathrm{~K}$ to $500 \mathrm{~K}$, with a step of $50 \mathrm{~K}$, including the $T_{w}=600 \mathrm{~K}$ case at the end.

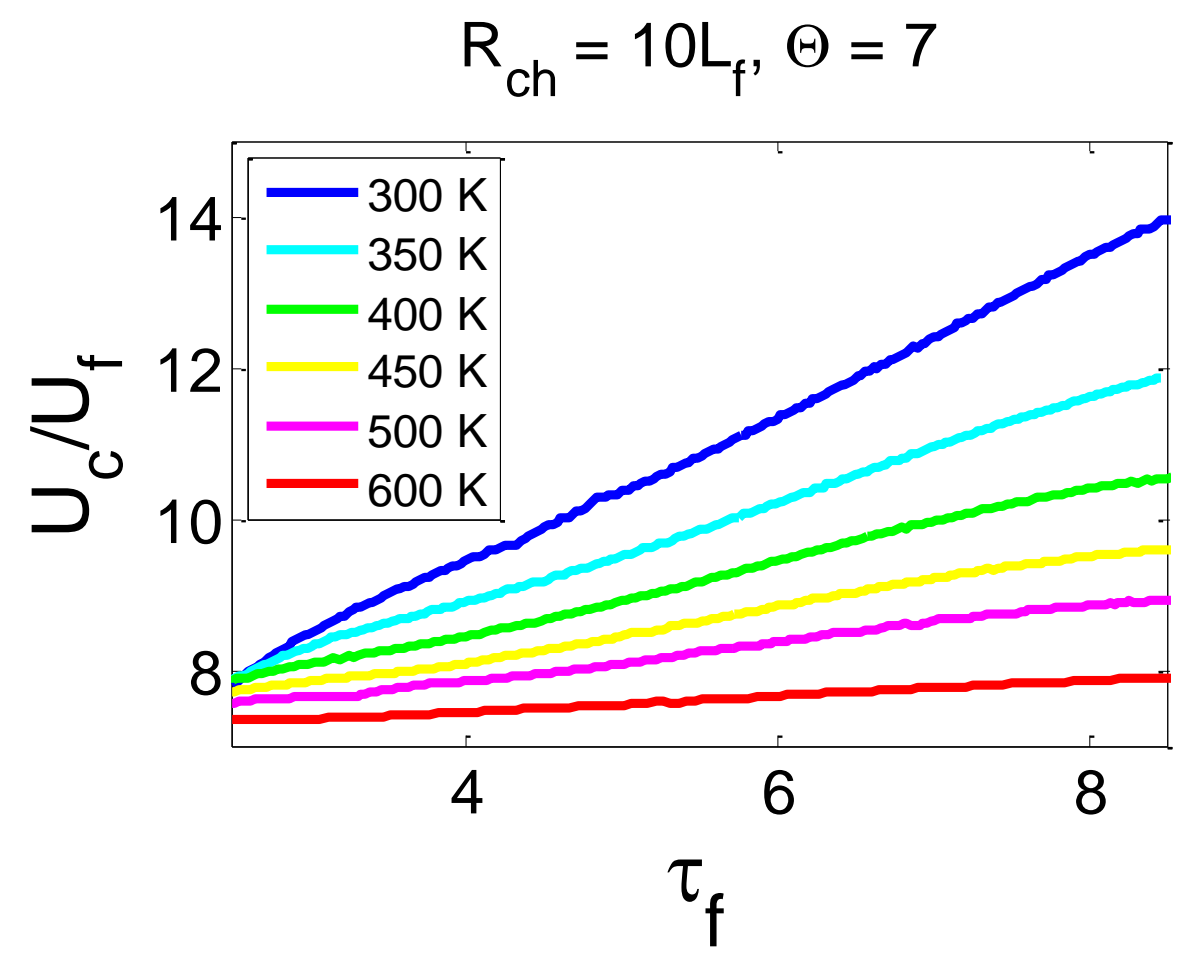

Figure 4.8. Linear acceleration regime at different wall temperatures. 
By quantifying the change of this linear acceleration in relation to the thermal conditions, namely, establishing a relation between the slopes of the velocity trend (roughly given by $U_{c} / U_{f}=a\left(\tau_{f}\right)+$ const.) and the associated wall temperature (Figure 4.9), it is observed that the tendency of this change is rather quadratic than linear. For example, it is possible to have a $50 \%$ velocity reduction just by rising the temperature from $300 \mathrm{~K}$ to $400 \mathrm{~K}$, with a quasi-constant flame velocity when the wall temperature is in the vicinity of $600 \mathrm{~K}$.

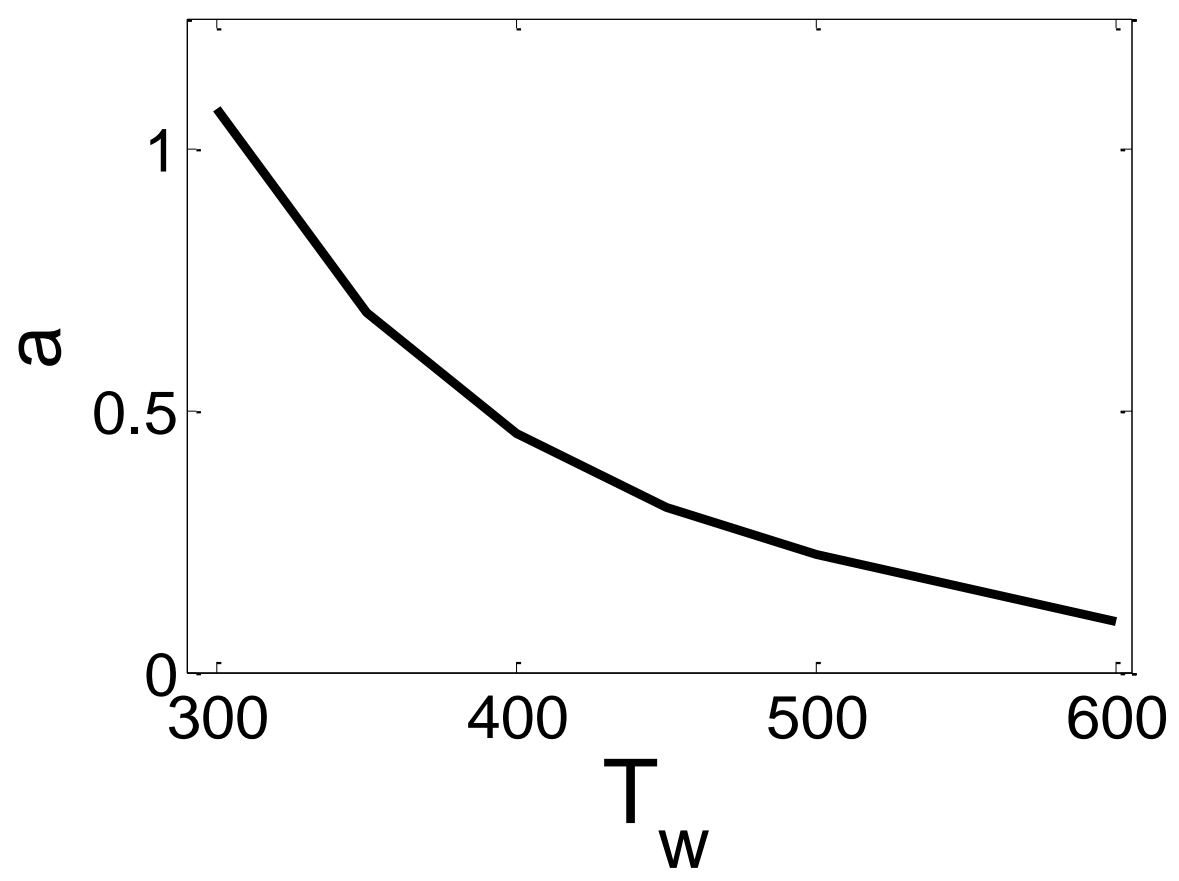

Figure 4.9. Slopes of the linear acceleration observed in the early stages of flame propagation for the cases presented in Figure 4.8.

As observed in Figure 4.7, a third characteristic trend occurs when the wall temperature is further increased. An oscillating behavior of the flame velocity is reached when the wall temperatures are kept close to the ignition temperature (e.g. $800 \mathrm{~K}$, red curve in Figure 4.7). As it has been demonstrated, the flame propagation velocity is proportional to the flame front shape. This case is not the exception. 


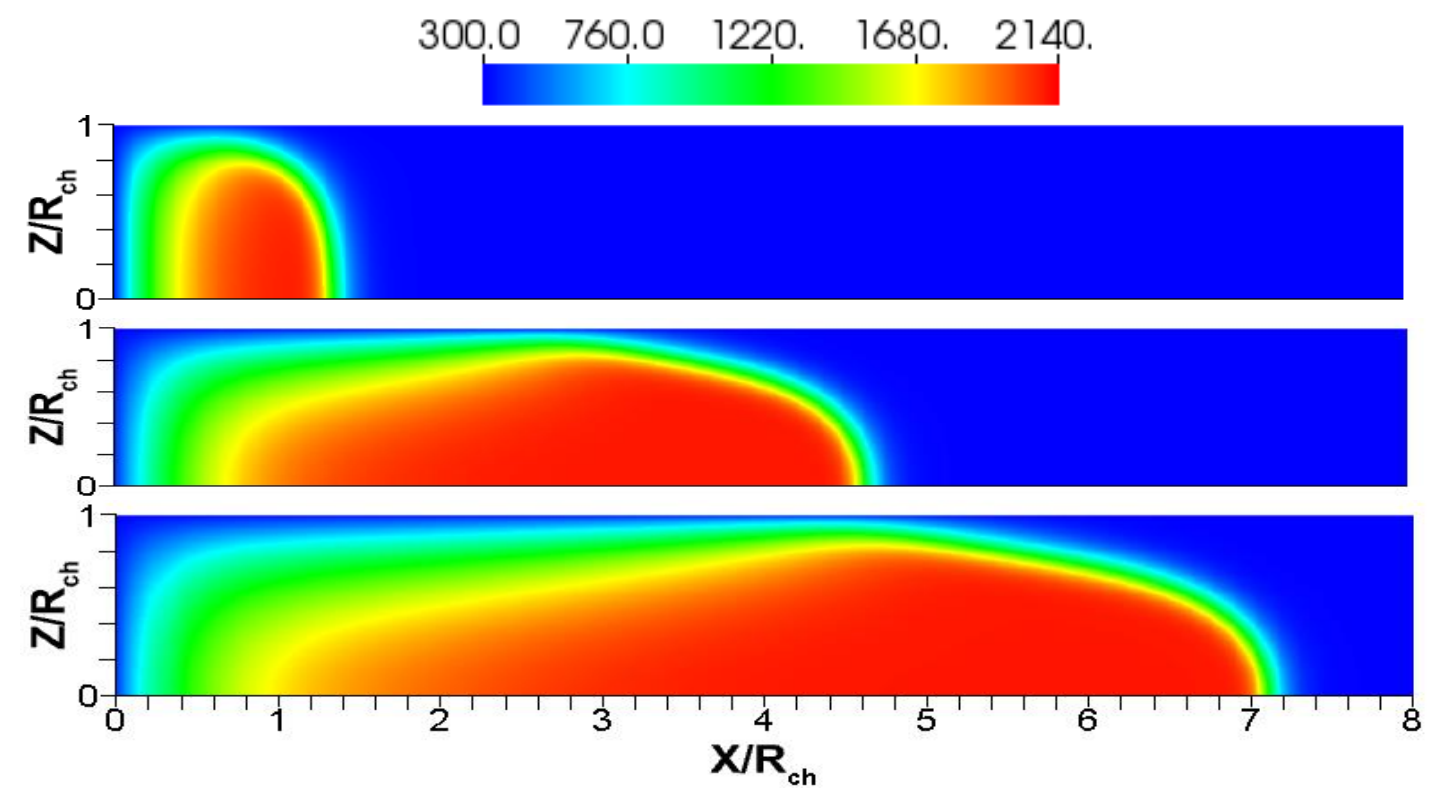

a)
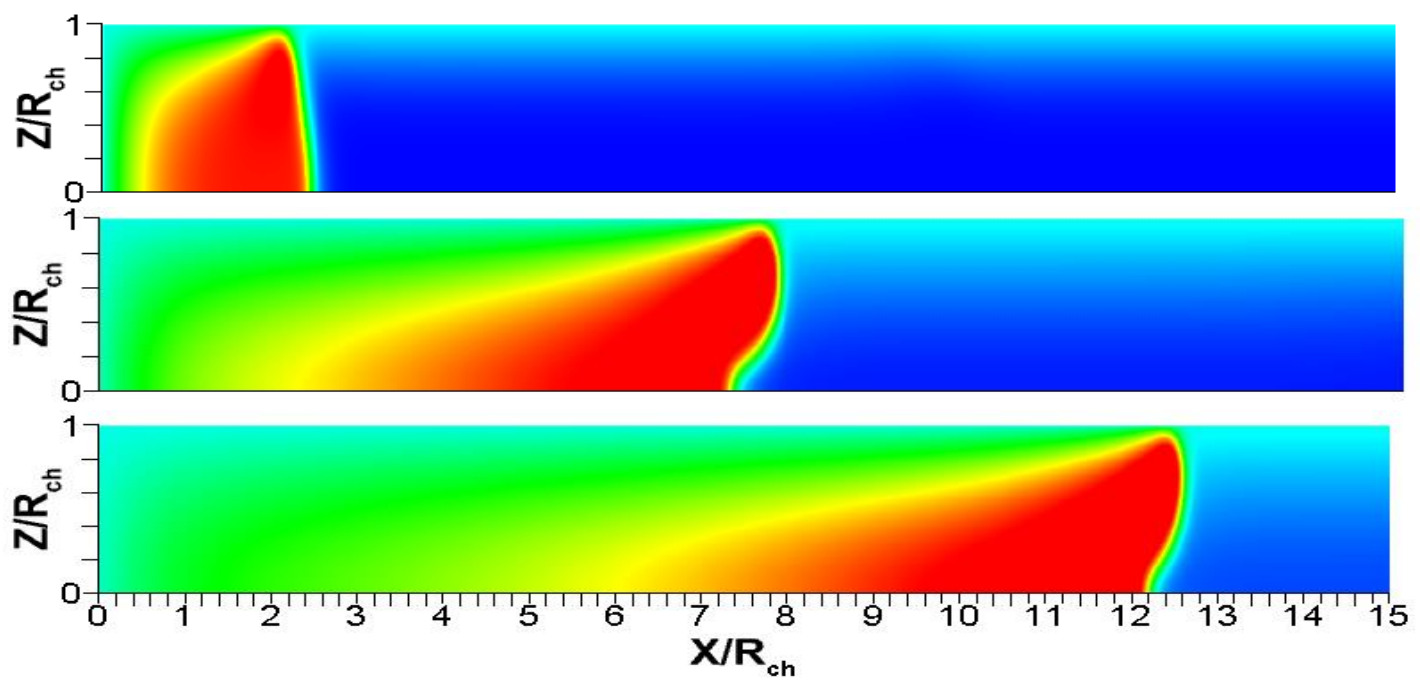

b)

Figure 4.10. Temperature distribution of the flame propagation in channels (upper half shown) with $\Theta=7, \mathrm{R}_{c h}=10 L_{f}$ and different wall temperatures a) $\mathrm{T}_{W}=300 \mathrm{~K}$, b) $\mathrm{T}_{W}=800 \mathrm{~K}$.

Two flame evolutions are shown in Figure 4.10, with the same conditions in both cases except for the channel wall temperature. First, the propagation attained when the cold wall condition $300 \mathrm{~K}$ is in place (Figure 4.10a), and second, when the wall temperature is raised to $800 \mathrm{~K}$ (Figure 4.10b). It is noticed that the flame propagation in the colder channel, Figure 4.10a, is characterized by a shape of the flame front that remains constant after an initial growth, with the flame tip located at the center of the channel. On the contrary, the flame propagating in the 
channel with $T_{w}=800 \mathrm{~K}$, Figure $4.10 \mathrm{~b}$, exhibits a changing behavior of the flame front. The flame tip location moves from the centerline of the channel to the wall. In addition, rather than intensifying the propagation near the wall, the flame is slightly contracted, acquiring a more planar shape. In this case, the hotter region near the walls facilitates the burning process, enhancing the propagation in this region. But the enhanced combustion needs to overcome the volumetric expansion of the burned matter, which is hotter at the center of the channel, producing the observed flame front contraction.

It looks that if the wall temperature is further increased, then the enhancing effect near the wall will be strong enough to keep the flame elongation along the wall and, consequently, making the flame acceleration sustained therein. This observation moves the analysis to consider very hot wall conditions, as presented next.

\subsubsection{Very High Wall Temperature Conditions}

A further increasing in the wall temperature to very high values, such as $T_{w}=1200 \mathrm{~K}$, is an interesting scenario from the fundamental point of view, though this temperature would be hardly attained in the practical reality and may not have associated applications. Indeed, while extremely hot wall temperatures may surpass the thermal limitations of most materials used in experiments, or those to be used in common applications, it provides a helpful scenario for the fundamental understanding of flame dynamics. Figure 4.11 shows the flame evolution in a 2D channel with $T_{w}=1200 \mathrm{~K}$. Here, the propagation is produced in a channel twice the width of the previous cases, therefore a direct comparison is not undertaken.

In this case, the thermal energy transferred to the unburned gas mixture near the wall plays the dominant role; it prevails over the hydraulic resistance which tends to restrain the propagation in the region. Instead, the flame shape typically given by the combination of frictional forces at the walls and the volumetric expansion in the central region is replaced by that associated with the flame spreading near the wall. Unlike the case described in Figure 4.10b, here the flame propagation does not moderate, and the associated stretching is considerably larger. However, as it will be shown in Section 4.1.5, this mechanism of flame acceleration is limited in time. Since the flame front expands not only horizontally, but vertically, collapsing in the center with its counterpart coming from below. The result is a major reduction of the flame surface, and consequently, a deceleration of the flame propagation. An interesting feature observed in this 
case is the generation of a 'spontaneous' combustion near the wall, away from the flame front. This event is not surprising, given the large temperature imposed at the walls, although it is not necessarily easy to predict in terms of time and location.

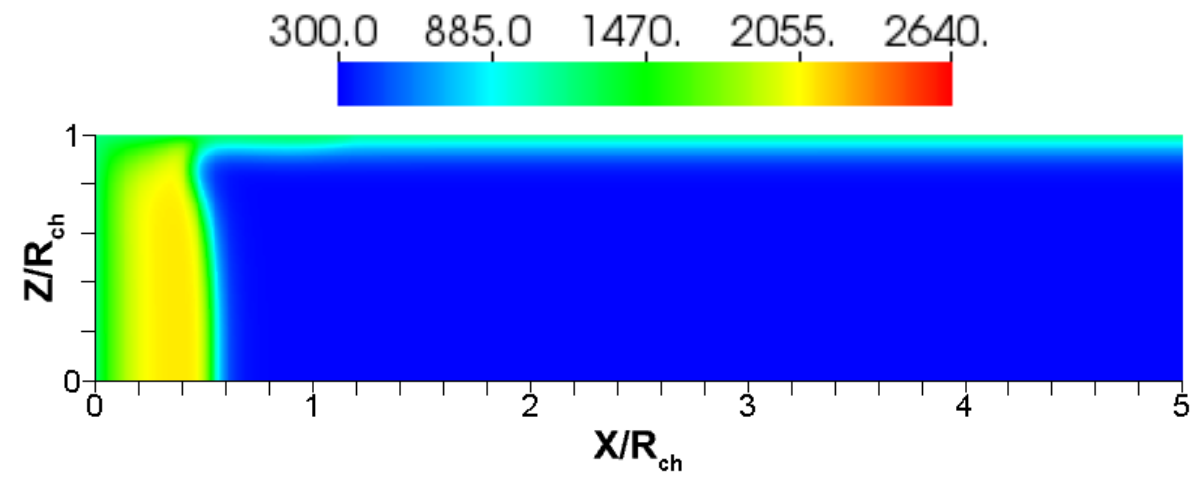

a)

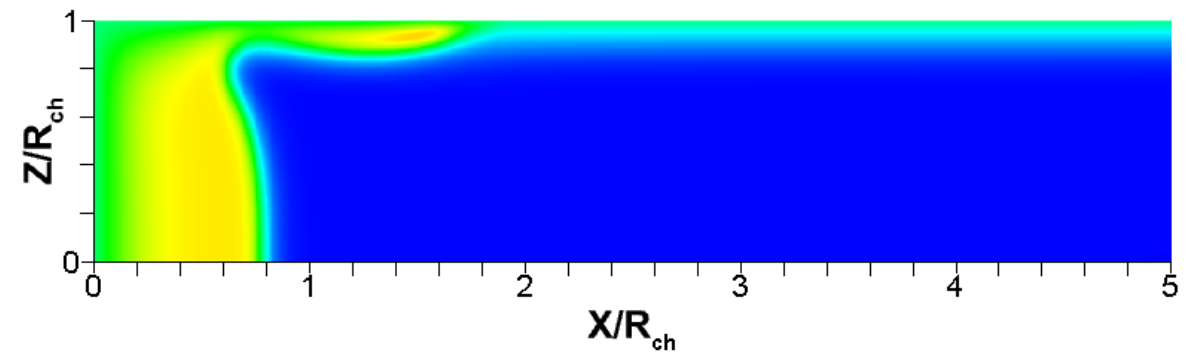

b)

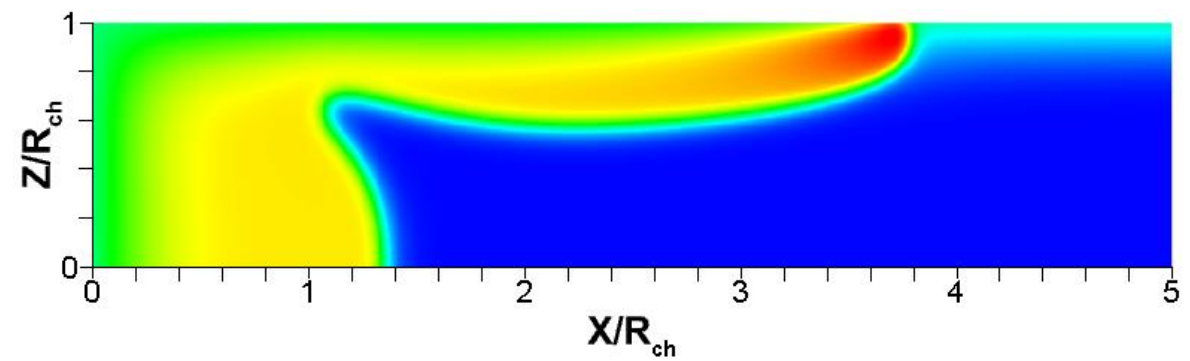

c)

Figure 4.11. Temperature distribution of the flame propagation in a channel (upper half shown) with walls at $\mathrm{T}_{W}=1200 \mathrm{~K}, \Theta=7, \mathrm{R}_{c h}=20 L_{f}$. a) $\tau_{f}=0.674$, b) $\tau_{f}=1.24$, c) $\tau_{f}=2.42$.

The precise instant when the spontaneous combustion is produced at the wall is captured in Figure 4.12. The fresh mixture is ignited in a region away from the propagating flame front, as noticed by the disconnected contour corresponding to $1560 \mathrm{~K}$. The large thermal energy given by the very hot wall is also observed by means of the shape of the temperature contours behind the spontaneous combustion region, where they stretch toward the region parallel to the wall. 


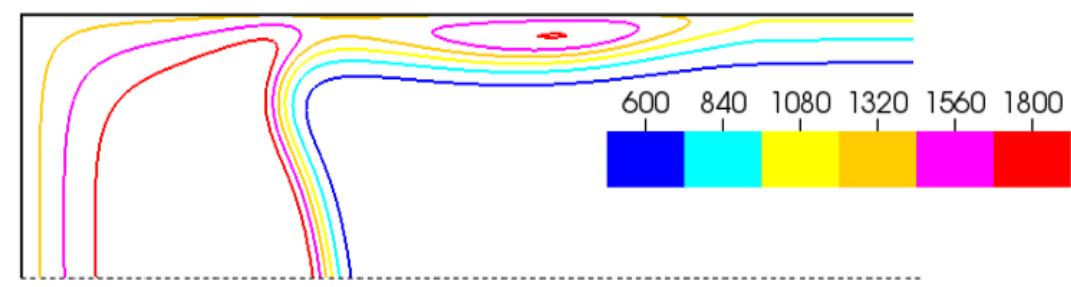

Figure 4.12. Temperature contours in a channel with walls at $\mathrm{T}_{W}=1200 \mathrm{~K}, \Theta=7, \mathrm{R}_{c h}=20 L_{f}$.

The fluid mechanics associated with high wall temperatures is also particular. The burned gas expands along the wall, supporting the flame propagation in this region. Also, a source-like flow is observed at the flame front, where the flame stretching tendency is shifted (Figure 4.13). This corresponds to a change in the momentum transfer direction: below this location, the burned gas expansion resumes the behavior observed in channels subjected to colder wall temperatures, which are primary driven by the thermal expansion of the hotter gas at the centerline.

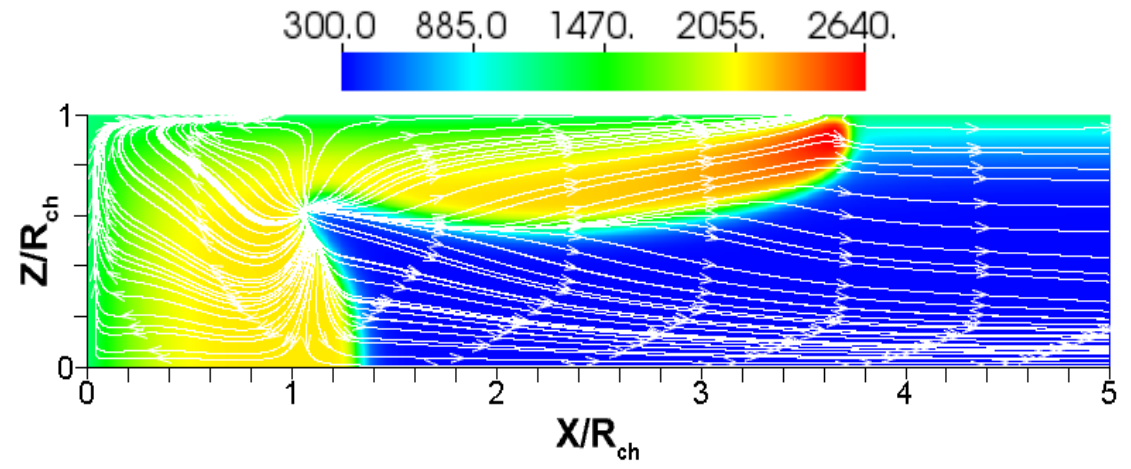

Figure 4.13. Temperature distribution and streamlines of the flame propagation in a channel (upper half shown) with walls at $\mathrm{T}_{W}=1200 K, \Theta=7, \mathrm{R}_{c h}=20 L_{f}$.

In this section, the wall temperature effect on the flame propagation has been investigated. This effect has been studied by keeping certain parameters constant, such as the channel width (with exception of the last case) and fuel mixture properties. Now, the analysis is extended to include the role of the fuel mixture on the combustion course under these channel conditions. This is performed by varying the thermal expansion coefficient, which is inherent to the fuel mixture.

\subsubsection{Thermal Expansion Effect on the Flame Propagation Dynamics}

The thermal expansion coefficient $\Theta$, determines the density drop experienced at the flame front, and approaches the ratio of the burned matter temperature to the fuel mixture temperature $(\Theta=$ $T_{b} / T_{f}$ ), when the flame velocity is much less than the speed of the sound (i.e. when the quasi- 
isobaric and ideal-gas approaches are adoptable), and the burned and unburned molar gases are the same. The thermal expansion is coupled to the specific energy released in the reaction as:

$$
Q=c_{p} T_{f}(\Theta-1)
$$

It is seen that this parameter modifies the flame velocity by determining the burned gas temperature and thermal energy released as a result of the chemical reaction occurring within the flame front. However, when interacting with isothermal wall conditions, the effect of the thermal expansion can become less apparent. For example, one could expect that given a larger thermal expansion value, the larger energy release associated with it will promote the flame propagation at any circumstance. This conclusion is disproved by Figures 4.14 and 4.15, where two channels, with isothermal walls kept at $300 \mathrm{~K}$, contain two fuel mixtures characterized by the thermal expansion coefficients $\Theta=5$ and $\Theta=7$. Indeed, the flame propagation is possible when $\Theta=5$ only, whereas an extinction event is observed for the fuel mixture characterized by $\Theta=7$.

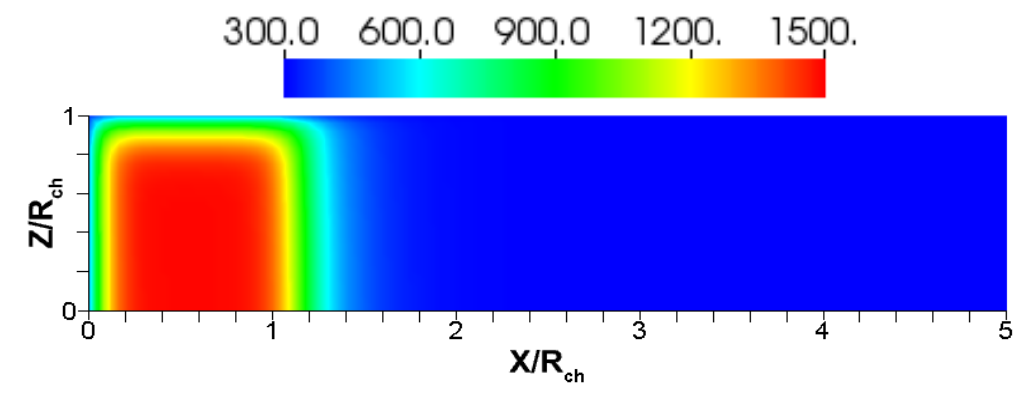

a)

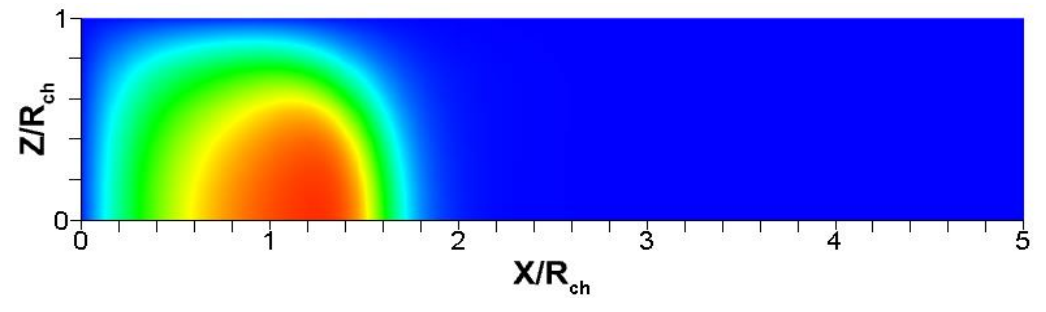

b)

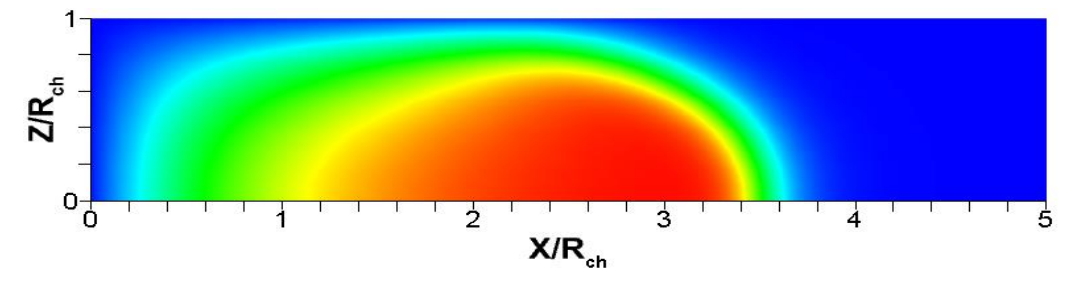

c)

Figure 4.14. Temperature distribution of the flame propagation in channels (upper half shown) at different times a) $\tau_{f} \sim 0$, b) $\tau_{f} \sim 1.225$, c) $\tau_{f} \sim 2.925 . \Theta=5, \mathrm{R}_{c h}=5 L_{f}, \mathrm{~T}_{W}=300 \mathrm{~K}$. 


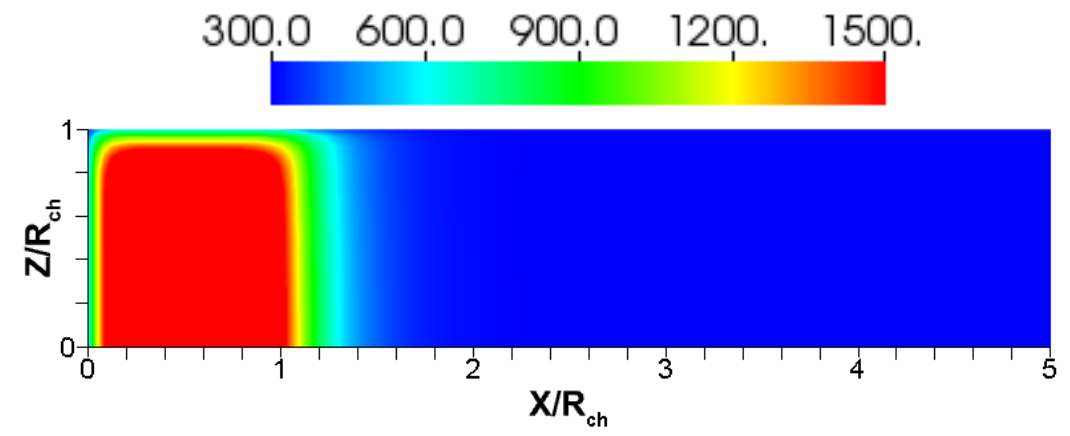

a)

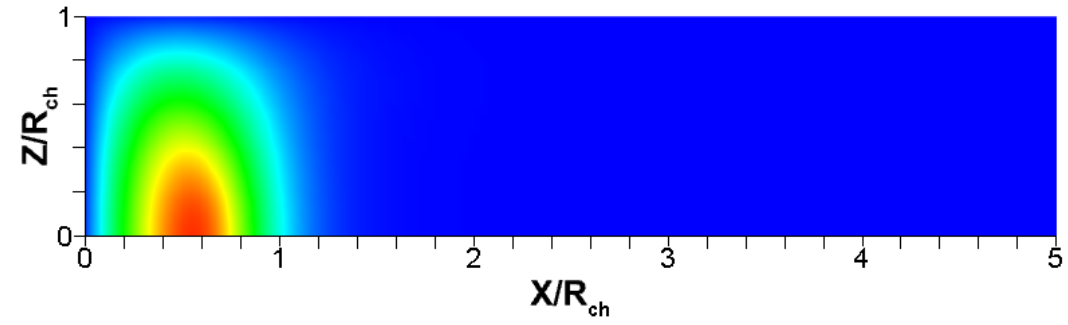

b)

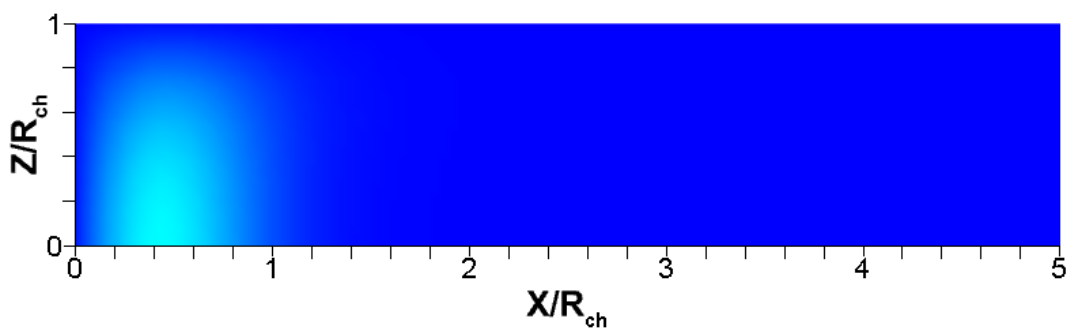

c)

Figure 4.15. Temperature distribution of the flame propagation in channels (upper half shown) at different times a) $\tau_{f} \sim 0$, b) $\tau_{f} \sim 1.1$, c) $\tau_{f} \sim 2.27 . \Theta=7, \mathrm{R}_{c h}=5 L_{f}, \mathrm{~T}_{W}=300$.

It should be mentioned that Figures 4.14 and 4.15 correspond to the narrowest channel considered in this investigation, $\mathrm{R}_{c h}=5 L_{f}$. Therefore, it is not surprising that in this dimensions the overall heat lost provides conditions for combustion extinction, since it surpasses the flammability limit found in regions around six times the laminar flame thickness $\left(\sim 6 L_{f}\right)$ apart from a cold wall $[44,62]$. However, such a limit is not reached in a more "colder" environment, as determined by a lower thermal expansion coefficient, where the propagation is possible (Figure 4.16). 


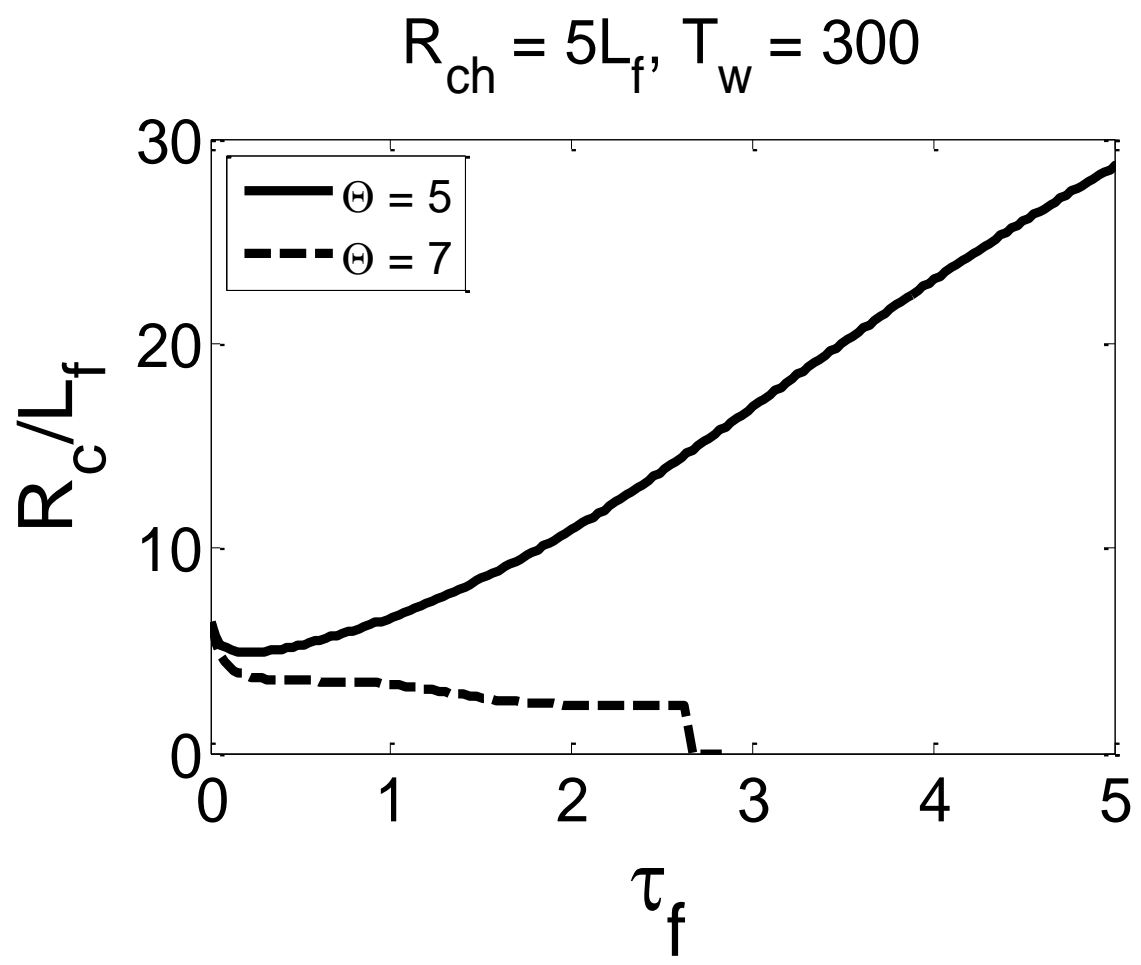

Figure 4.16. Evolution of the flame position along the channel centerline for flame extinction $(\Theta=7)$ and propagation $(\Theta=5)$ cases.

The reason for having a sustained combustion process in a less 'energetic' environment relies on the heat transfer process. A lower thermal expansion generates a cooler burned matter, as seen in the snapshots of Figures 4.14 and 4.15. Keeping in mind that the wall temperature does not vary with time, the heat lost is basically modified by the burned gas temperature, thus, $\mathrm{q}_{\text {lost }}=$ $\mathrm{h}_{w}\left(\mathrm{~T}_{b}-\mathrm{T}_{w}\right)=\mathrm{h}_{w}\left(\Theta \mathrm{T}_{f}-\mathrm{T}_{w}\right)$, with $\mathrm{h}_{w}$ the heat transfer coefficient between the wall and burned gas. In this case, the fuel mixture and the wall temperature are at room temperature $300 K$, leading to $\mathrm{q}_{\text {lost }}=\mathrm{h}_{w}(\Theta-1) \mathrm{T}_{\text {room }}$. For $\Theta=7$, the associated heat lost is given by $\mathrm{q}_{\text {loss }, \Theta=7}=6 \mathrm{~h}_{w} \mathrm{~T}_{\text {room }}$, whereas for $\Theta=5$ by $\mathrm{q}_{\text {loss }, \Theta=5}=4 \mathrm{~h}_{w} \mathrm{~T}_{\text {room }}$, establishing a $\mathrm{q}_{\text {loss }, \Theta=7} / \mathrm{q}_{\text {loss }, \Theta=5}=1.5$ ratio between the heat lost in each scenario. The bigger losses obtained in the $\Theta=7$ case modifies the flame structure, leading it to flame quenching, whereas for $\Theta=5$ the heat lost also cools the flame front region down, but to a point where a recovery is possible, which occurs once the flame departs from the closed end, Figure 4.17. 


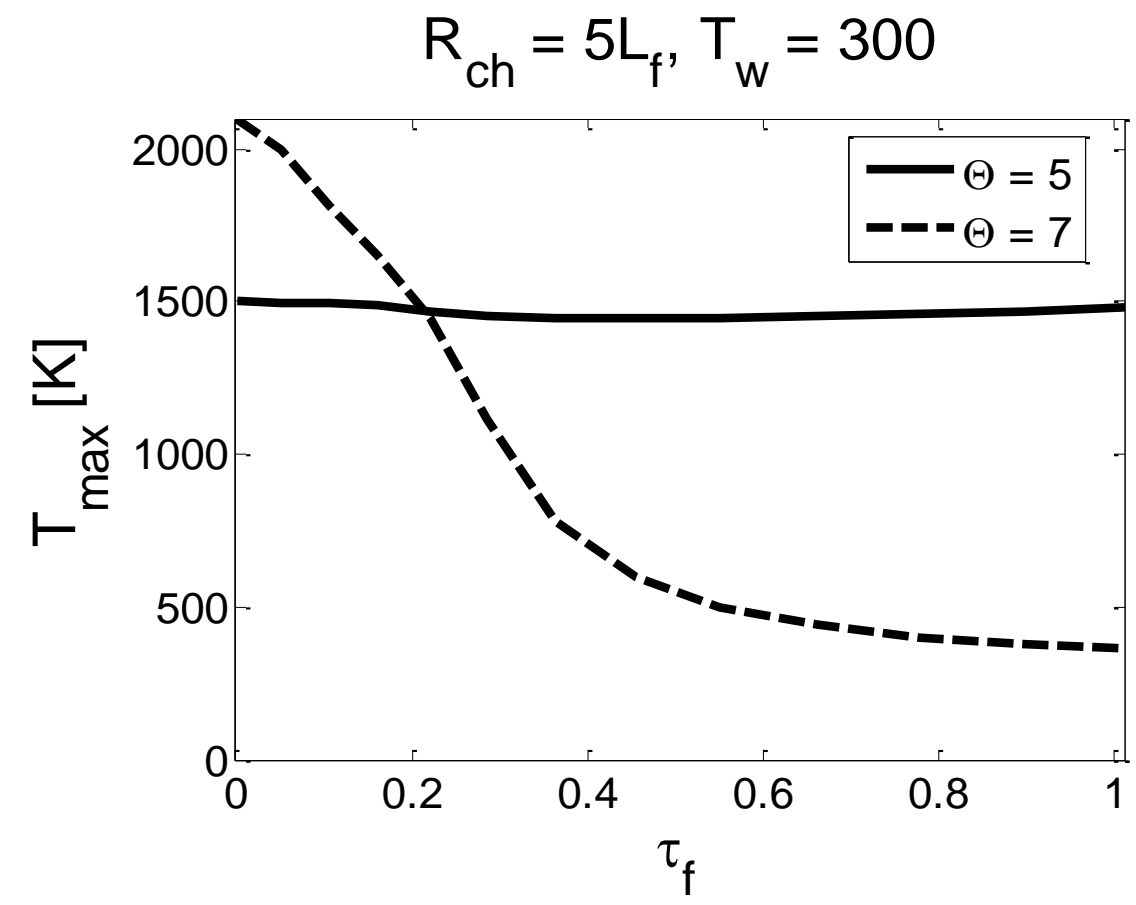

Figure 4.17. Maximum temperature registered in the channel along the process.

Since it is the burning process the main source of thermal energy in the system, much more than the energy transferred by the walls (i.e. $>90 \%$ of total heat if $T_{w}=900 \mathrm{~K}$ is in place), the thermal energy in the system is 'set up' by specifying the fuel thermal expansion. In this sense, the effects of isothermal walls on the flame morphology, observed in the previous section, are expected to occur at different thermal expansions as well, although at a different energy 'level', e.g. different wall temperature. The next section provides a good example of this situation.

\subsubsection{Enhancing Flame Propagation at the Walls}

It was shown in Section 4.1.2 that a high wall temperature can move the flame tip out of the channel centerline to place it near to the wall. Therein, an oscillating flame velocity behavior was identified in relation to a wall temperature value of $800 \mathrm{~K}$, channel half-width $R_{c h}=10 L_{f}$, and thermal expansion ratio $\Theta=7$. Figures 4.18 and 4.19 show the flame evolution for the same conditions, considering $\Theta=5$ and $\Theta=9$, respectively, which approach the effect of having two different fuel mixtures.

Figure 4.18 shows the flame propagation for $\Theta=5, T_{w}=800 \mathrm{~K}$ and $R_{c h}=10 L_{f}$. It is noticed that the snapshots of Figure 4.18 resemble the case described in Section 4.1.3, which is related to a much hotter wall and twice wider channel $\left(1200 \mathrm{~K}, R_{c h}=20 L_{c h}\right)$. In this case, however, there 
is no 'spontaneous' combustion near the wall. The flame progresses enhanced by the wall conditions, with the gases been more sensitive to the hot wall as a result of their 'lower' energy level (as compared to the $\Theta=7$ case, Figure 4.10b). This propagation progresses until the flame fills the channel width, which produces a substantial flame front surface reduction, and consequent flame propagation deceleration.

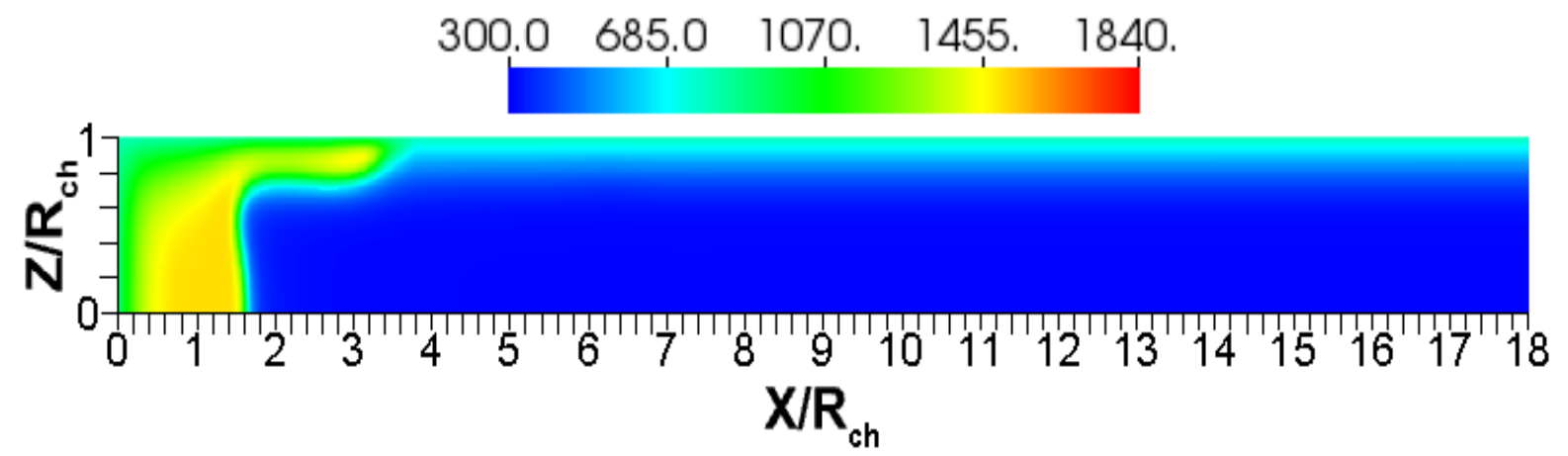

a)

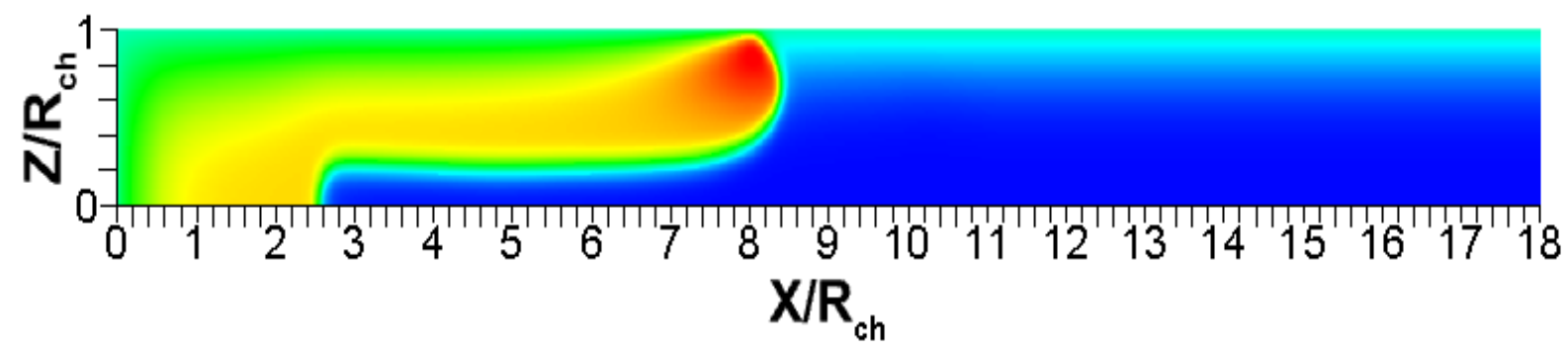

b)

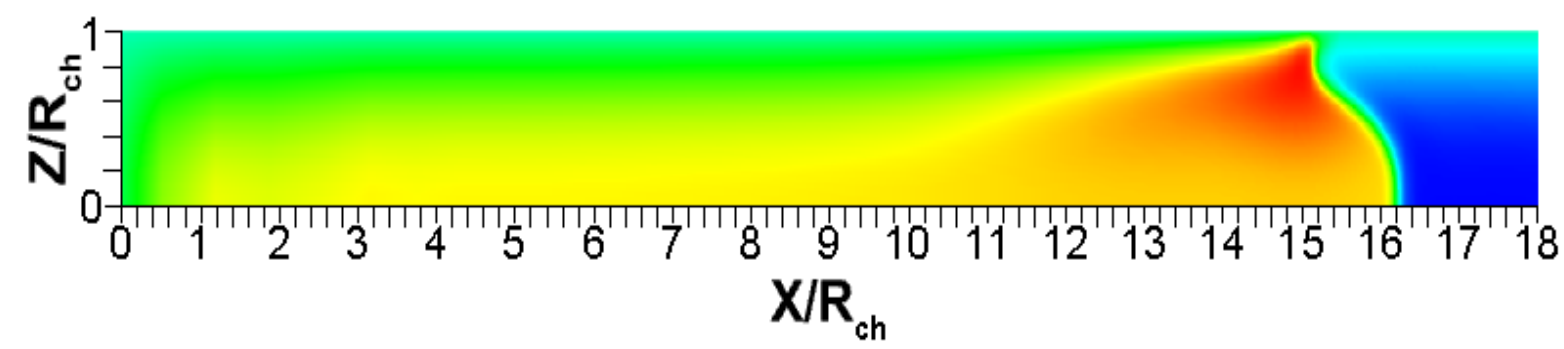

c)

Figure 4.18. Temperature distribution of the flame propagation in a channel (upper half shown) with walls at $\mathrm{T}_{W}=800 \mathrm{~K}, \Theta=5, \mathrm{R}_{c h}=10 L_{f}$. a) $\tau_{f} \sim 0.675$, b) $\tau_{f} \sim 1.45$, c) $\tau_{f} \sim 2.475$. 


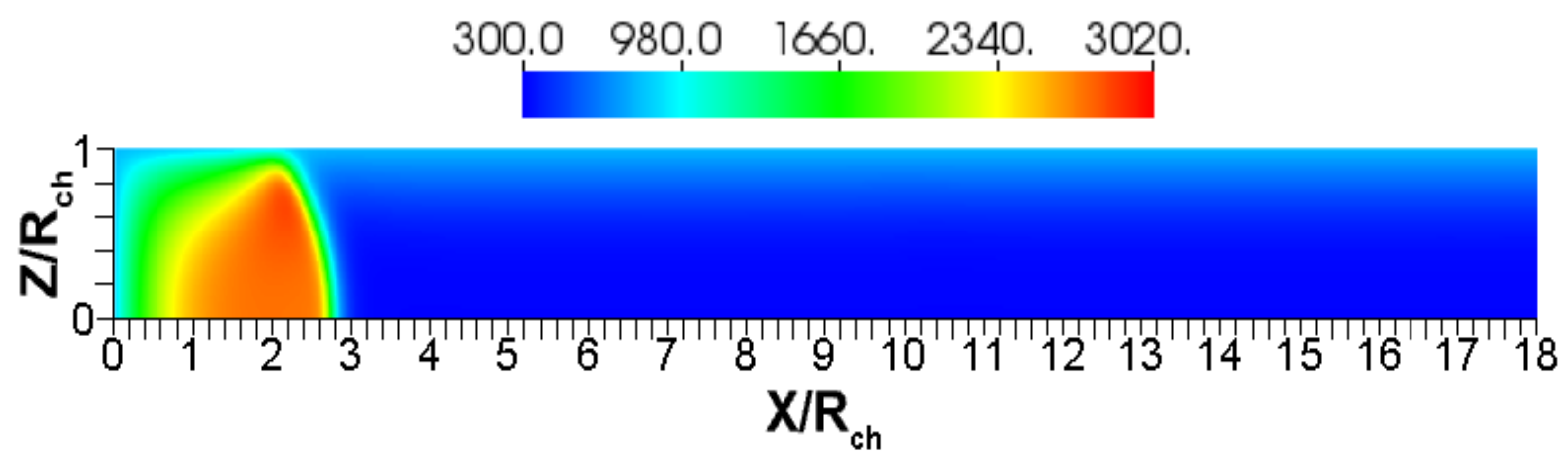

a)

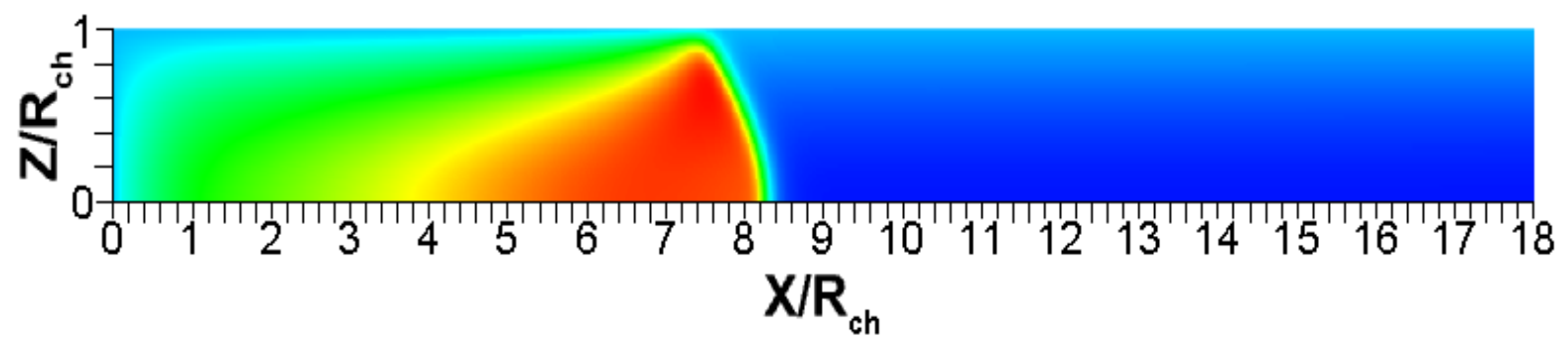

b)

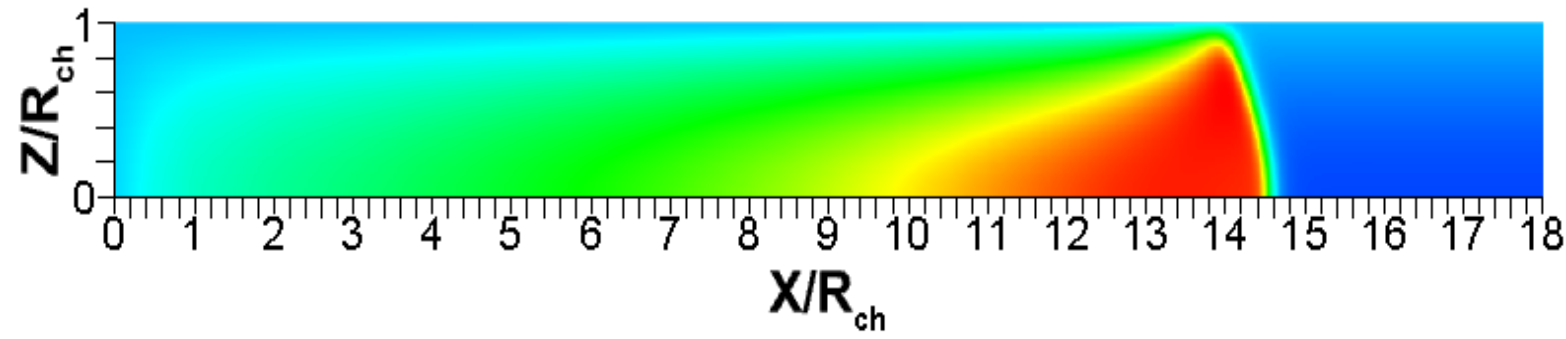

c)

Figure 4.19. Temperature distribution of the flame propagation in a channel with walls at $\mathrm{T}_{W}=$ $800 K, \Theta=9, \mathrm{R}_{c h}=10 L_{f}$. a) $\tau_{f} \sim 3.15$, b) $\tau_{f} \sim 9.99$, c) $\tau_{f} \sim 19.6$

On the contrary, by raising the thermal expansion to $\Theta=9$ (Figure 4.19), the flame propagation becomes more stable. Namely, the flame tip stays at the center of the channel, without significant variation of the flame front shape and surface area. A comparison of these two scenarios can be undertaken by looking at the flame front position along the channel in two different vertical locations: at the channel center and near the wall, as shown in Figures 4.20 and 4.21. 


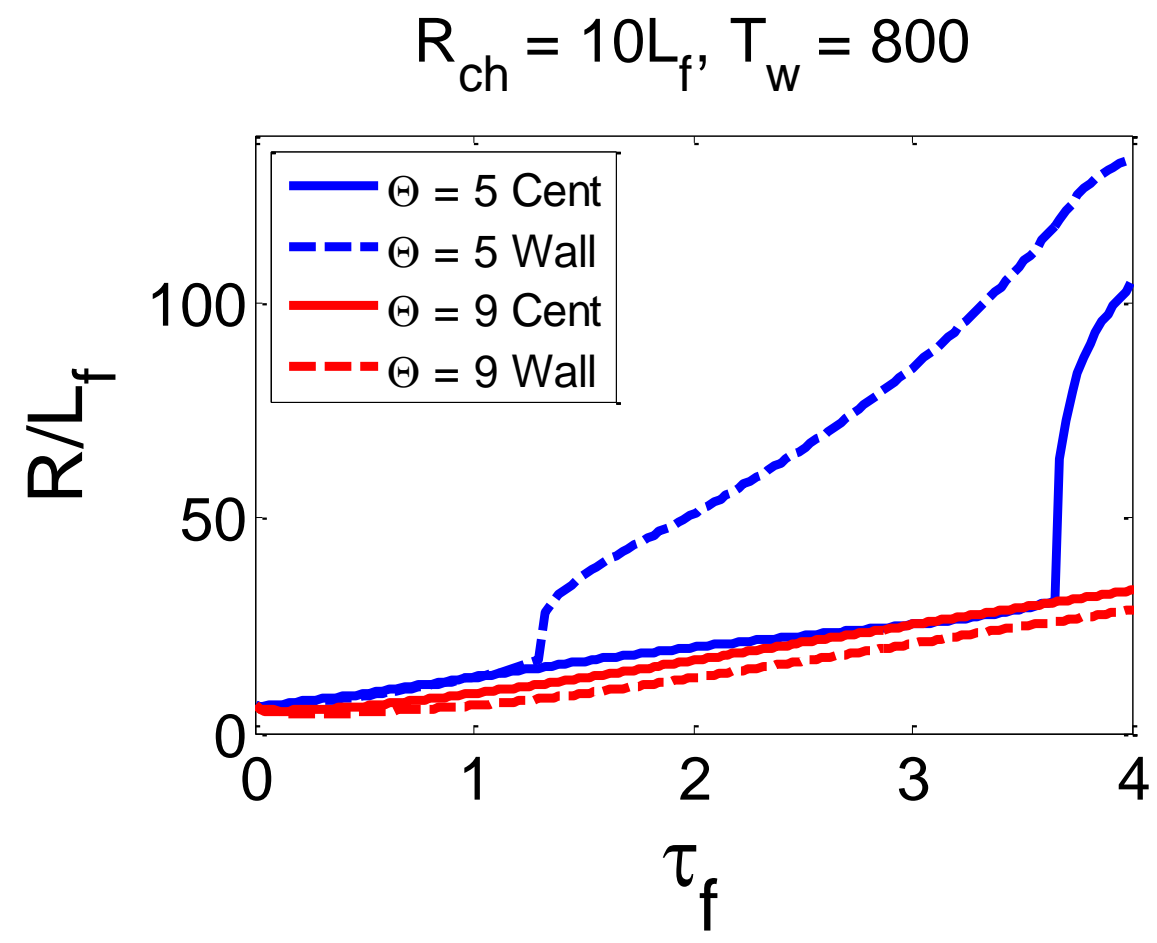

Figure 4.20. Flame position as reported at two locations, centerline and near the wall.

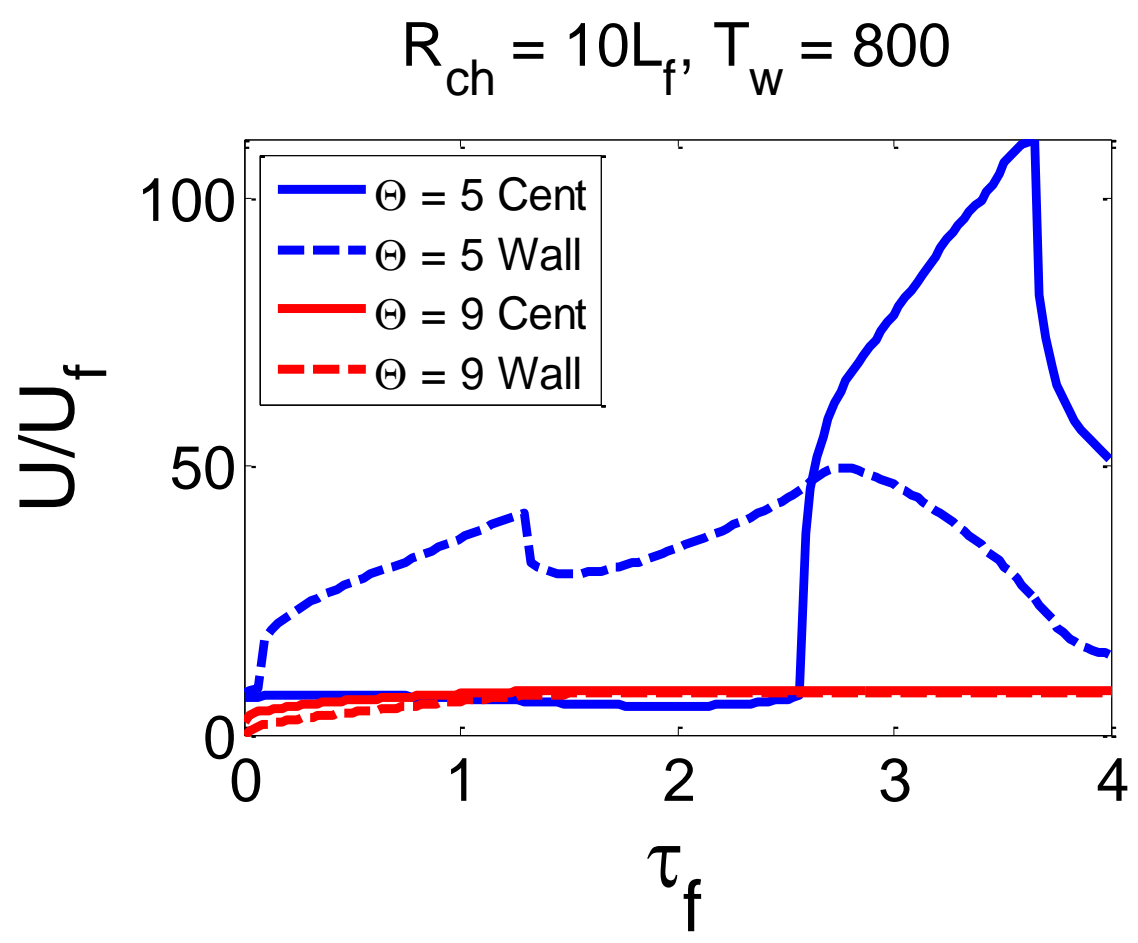

Figure 4.21. Flame velocity corresponding to the cases shown in Figure 4.20.

Figure 4.20 depicts how the flame propagation can proceed depending on the fuel mixture considered. The blue lines show the unsteady flame shape adopted when the effect of the heat transferred by the walls is enhanced by interacting with a low energy-release fuel mixture $(\Theta=$ 
5). The propagation in this case presents an erratic behavior, with the associated flame velocity reaching a peak value of 100 times the planar flame velocity (Figure 4.21). On the contrary, the burning of a fuel mixture characterized by a larger energy-release rate $(\Theta=9)$ generates almost no difference in the flame propagation speed at the two locations considered.

These results show how the fuel thermal expansion $\Theta$ can 'set up' the thermal energy in the system, modifying the wall thermal effects on the flame morphology and propagation. In addition, it governs one of the mechanisms of flame propagation, namely, the volumetric expansion of the burning gas, which is proportional to the density drop across the flame front. While such an expansion is produced uniformly in the burned gas region in adiabatic channels (restricted by the wall friction), this is not the case under isothermal conditions, since the burned gas includes horizontal and vertical temperature gradients. To better understand the effect of the thermal expansion in relation to the pushing force that it exerts on the flame front, the attention is restricted to conditions where the heat exchanged with the walls produces neither extinction nor stretching of the flame along the wall. For this reason, two channel sizes have been considered in Figure 4.22 and 4.23 , along with the cold wall condition $300 \mathrm{~K}$, where these situations are constructively avoided.

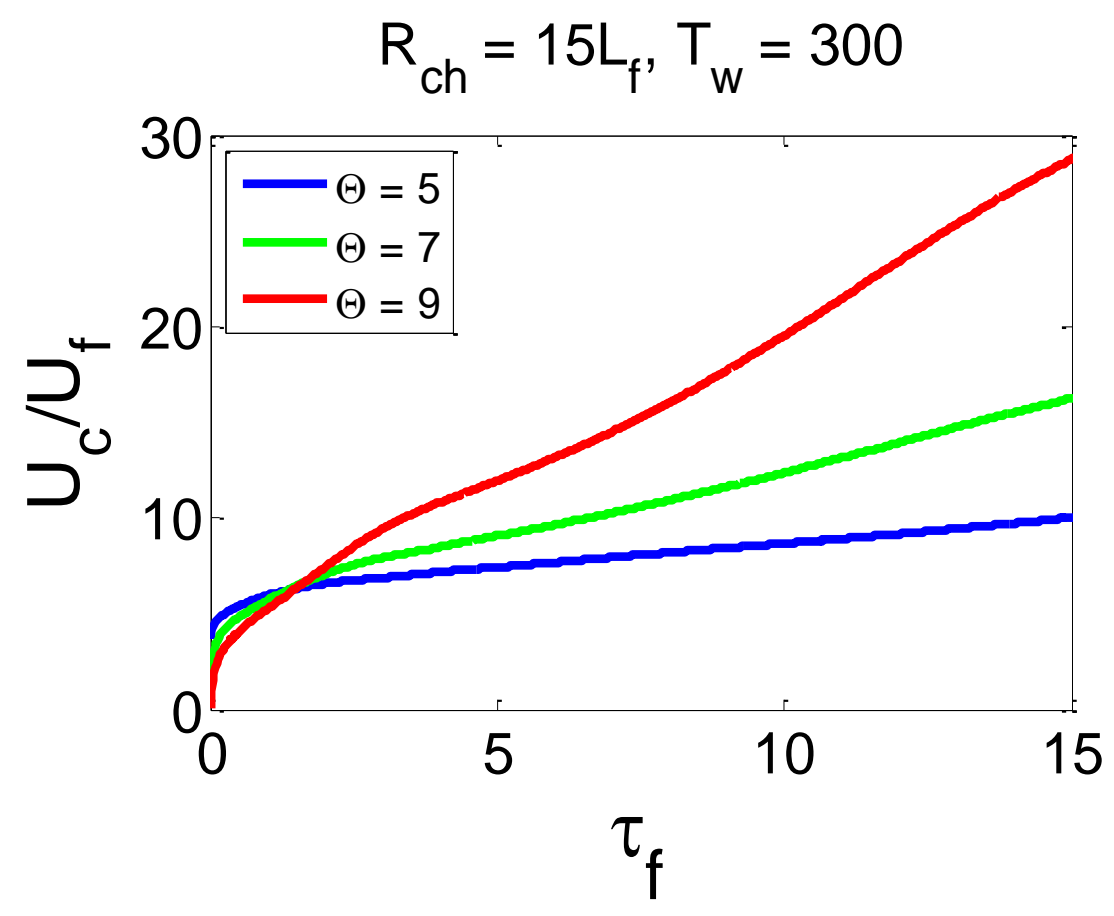

Figure 4.22. Flame velocity at the channel centerline for different thermal expansions. 


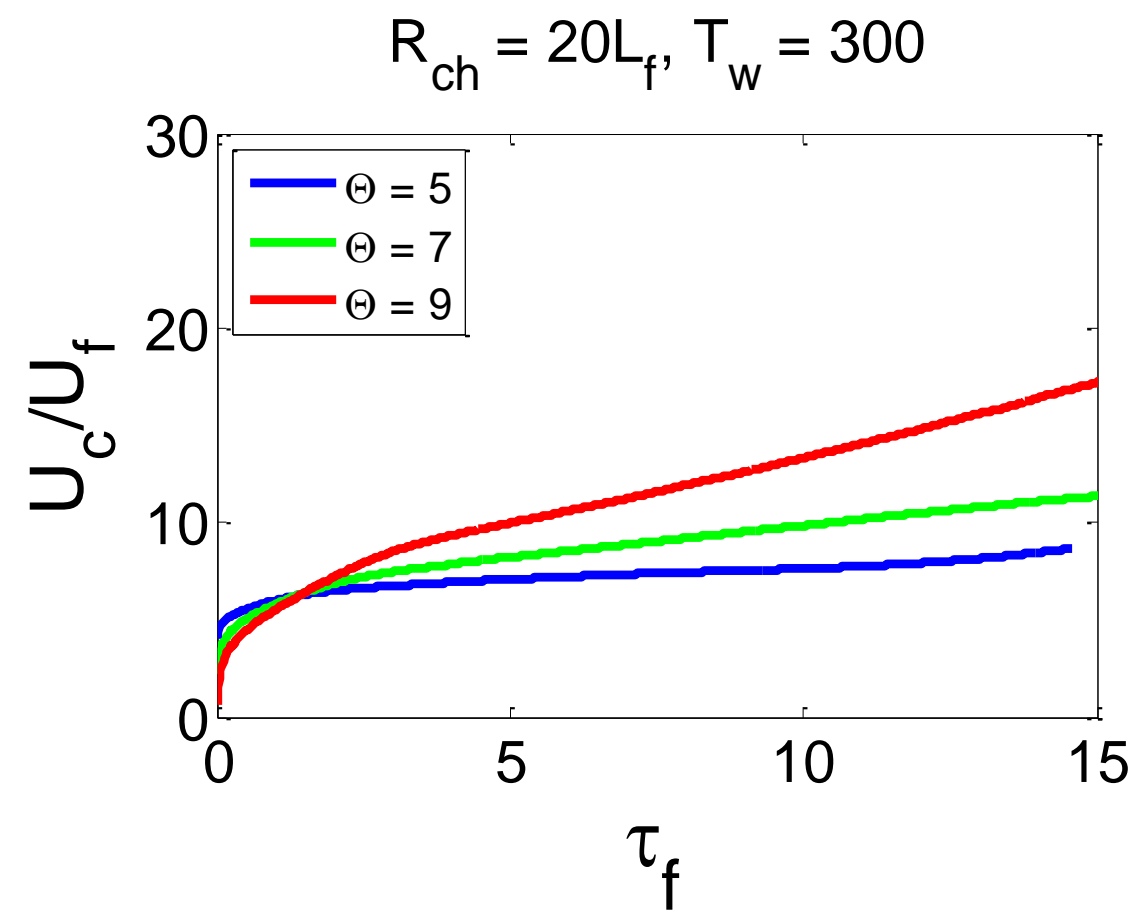

Figure 4.23. Flame velocity at the channel center for different thermal expansions.

The velocity trends shown in Figure 4.22 resemble that obtained at different wall temperatures for a fixed thermal expansion coefficient Figure 4.7 and 4.8. This confirms the role of the thermal expansion of the fuels, conditioning the isothermal wall effects on the combustion process. A more energetic system will suffer a more significant quenching of the flame front next to colder walls, which makes the flame front more corrugated. In addition, the flame propagation is further enhanced by the larger volumetric expansion associated with a larger $\Theta$. Figure 4.23 shows a comparison of the flame velocities associated with these three thermal expansion coefficients, at the same walls condition, but in a wider channel. The tendency is the same, with the flame propagating at a lower velocity. In fact, these results also reflect the effect of the variable investigated in the next section - the width of the channel.

\subsubsection{Channel Width Effect}

Previous studies have concluded that the flame propagation in isothermal channels strongly depends on the channel width. For example, hotter walls have been reported to promote faster flame velocities in tubes $6.4 \times 10^{-4} \mathrm{~m}$ wide, and vice versa in tubes two times wider [47]. These 
tendencies are observed in Figure 4.24 and Figure 4.25, where low and moderate wall temperatures are in place, $T_{w}=300 \mathrm{~K}$ and $500 \mathrm{~K}$, in channels of different widths.

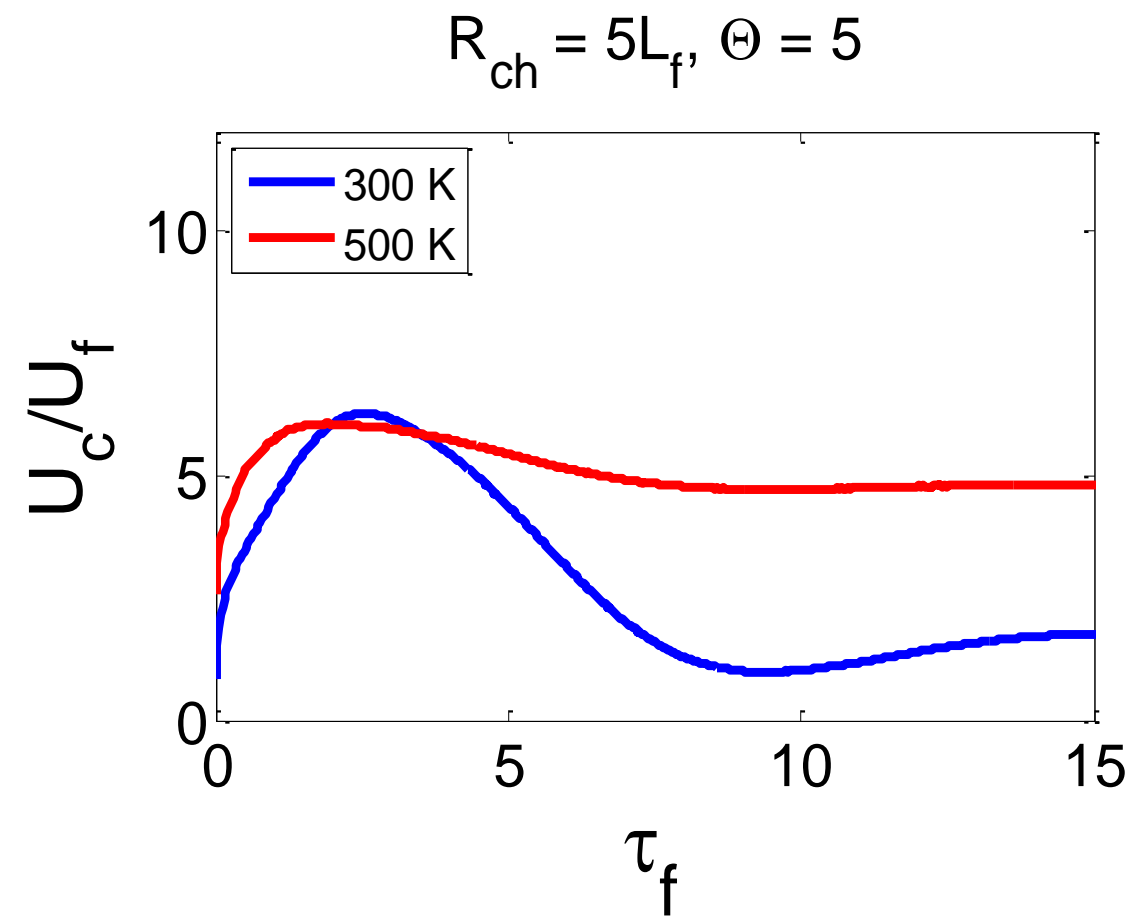

Figure 4.24. Flame velocity in channels of half width $5 L_{f}$, at two different wall temperatures.

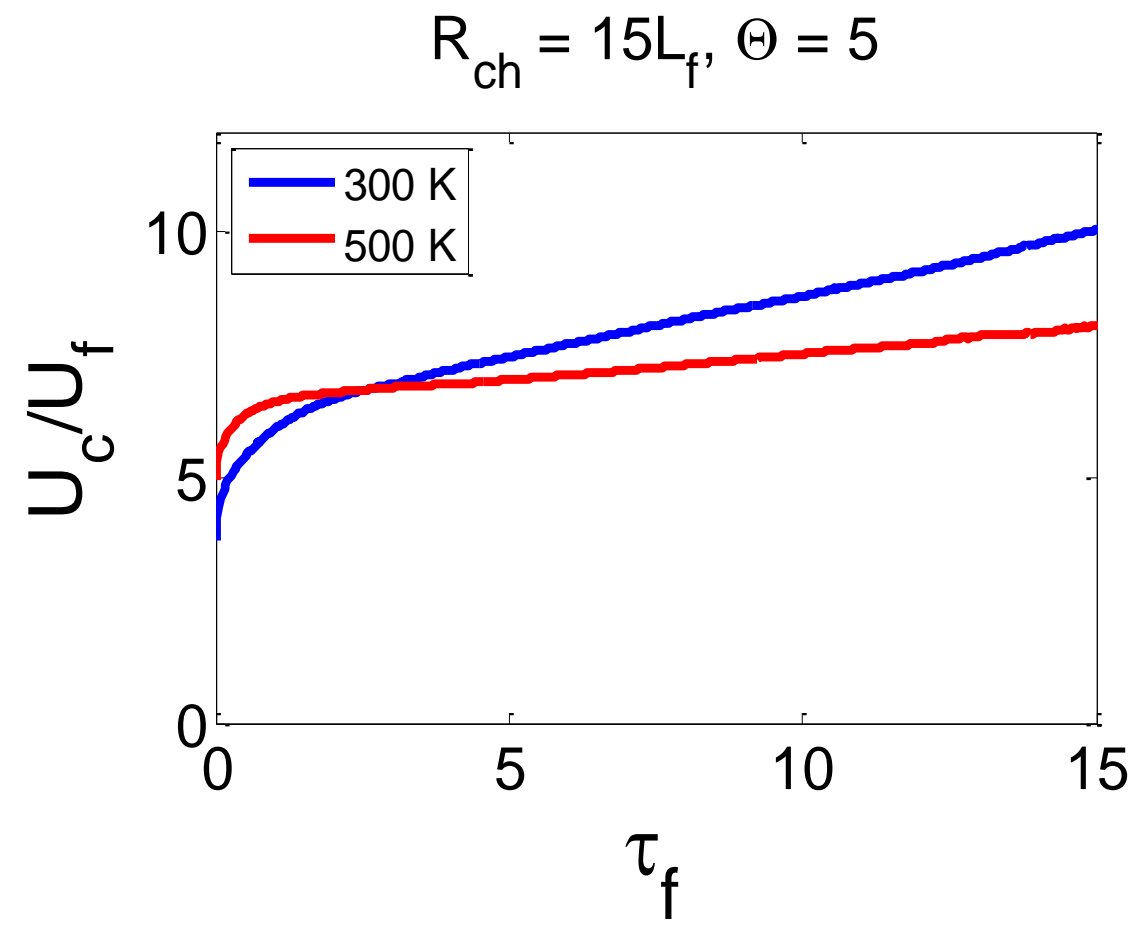

Figure 4.25. Flame velocity in channels of half-width $15 L_{f}$, at two different wall temperatures. 
In Figure 4.24, where a channel of half-width $5 L_{f}$ is considered, the flame propagation is faster for the hotter wall $T_{w}=500 \mathrm{~K}$ (as much as $80 \%$ faster), with exception of a short time interval, where $T_{w}=300 \mathrm{~K}$ wall promote a higher velocity (around $20 \%$ higher). With this channel width in place, the post-cooling of the burned gas is produced in a very efficient way, which would explain why this tendency is different from the observed, for instance, in Figure 4.25, which corresponds to a wider channel, $\mathrm{R}_{c h}=15 L_{f}$. Figure 4.25 reflects more closely the tendencies described in Section 4.1.2. In this case, as the pipe width increases, the burned matter is not easily cooled and the propagation is sustained for a longer period.

Figure 4.26 shows the results corresponding to an even wider channel, $\mathrm{R}_{c h}=30 L_{f}$, where the effect produced by the energy transfer at the walls becomes significantly less important. The flame propagation manner does not change with the wall temperature, such that the flame spreads with the same constant speed after a short time, independently of the wall temperature. Actually, it is not only the thermal effect at the walls that becomes less influential in the process, but the boundary conditions at the walls in general, including the frictional force. For instance, slower flame propagations have been reported as the tube and channel widths become bigger in adiabatic conditions [35, 36], because of the thinner boundary layer formed at the walls as compared to the channel section.

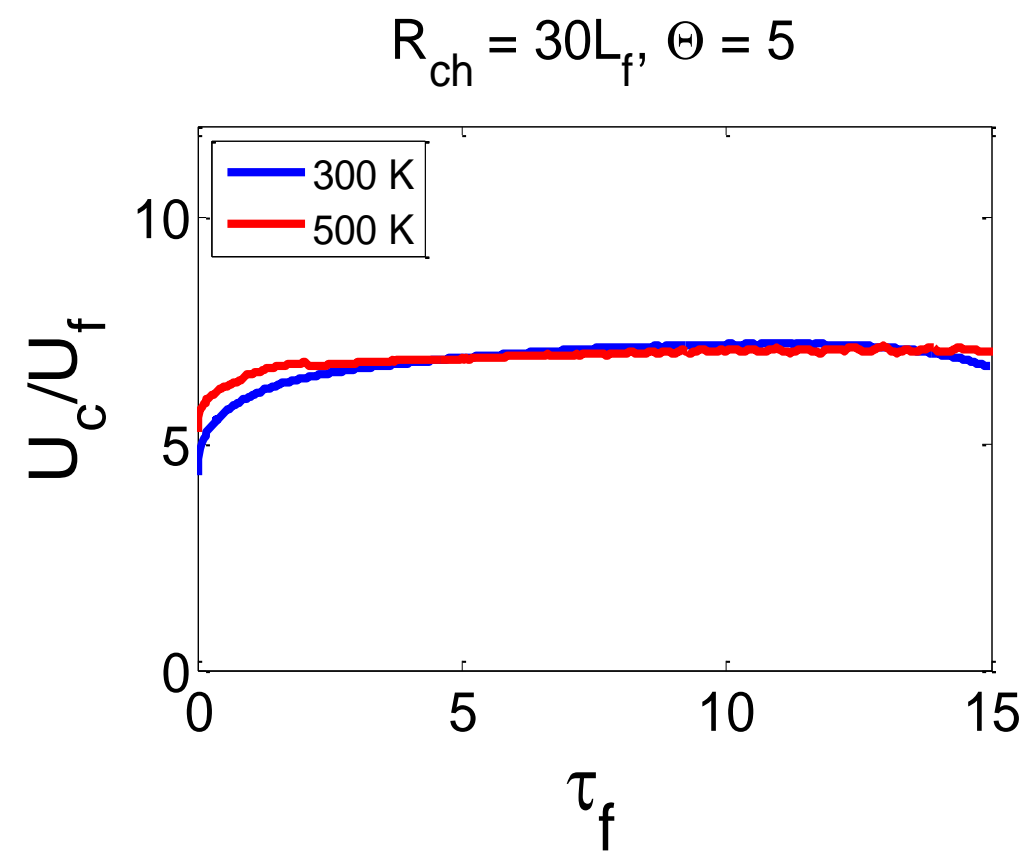

Figure 4.26. Flame velocity at the center of the channel, $\mathrm{R}_{c h}=30 L_{f}$, at two wall temperatures. 
So far, the strategy along this investigation has been to consider the effect of the multitude of variables involved in the parametric study in a separated manner, for the better understanding of the changes produced by them on the combustion process. At this point, it seems interesting to combine the variables in such a way that certain regimes can be established, where coinciding characteristic describing the burning process and propagation intensity are observed.

\subsubsection{Discussion}

Four regimes of the flame propagation have been revealed in isothermal channels, namely (i) no propagation or extinction; (ii) a linear acceleration stage of the flame; (iii) flame propagating at a minor acceleration rate or with an almost constant speed; and (iv) an oscillating behavior of the flame propagation velocity. These regimes have demonstrated a strong dependence on the wall temperature, channel width and on the fuel thermal expansion. Based on the results of this study, where the flame velocity has been computed as the flame propagated a distance $\sim 100 L_{f}-$ $150 L_{f}$ in the channel, these regions have been quantified as shown in Figures 4.27-31.

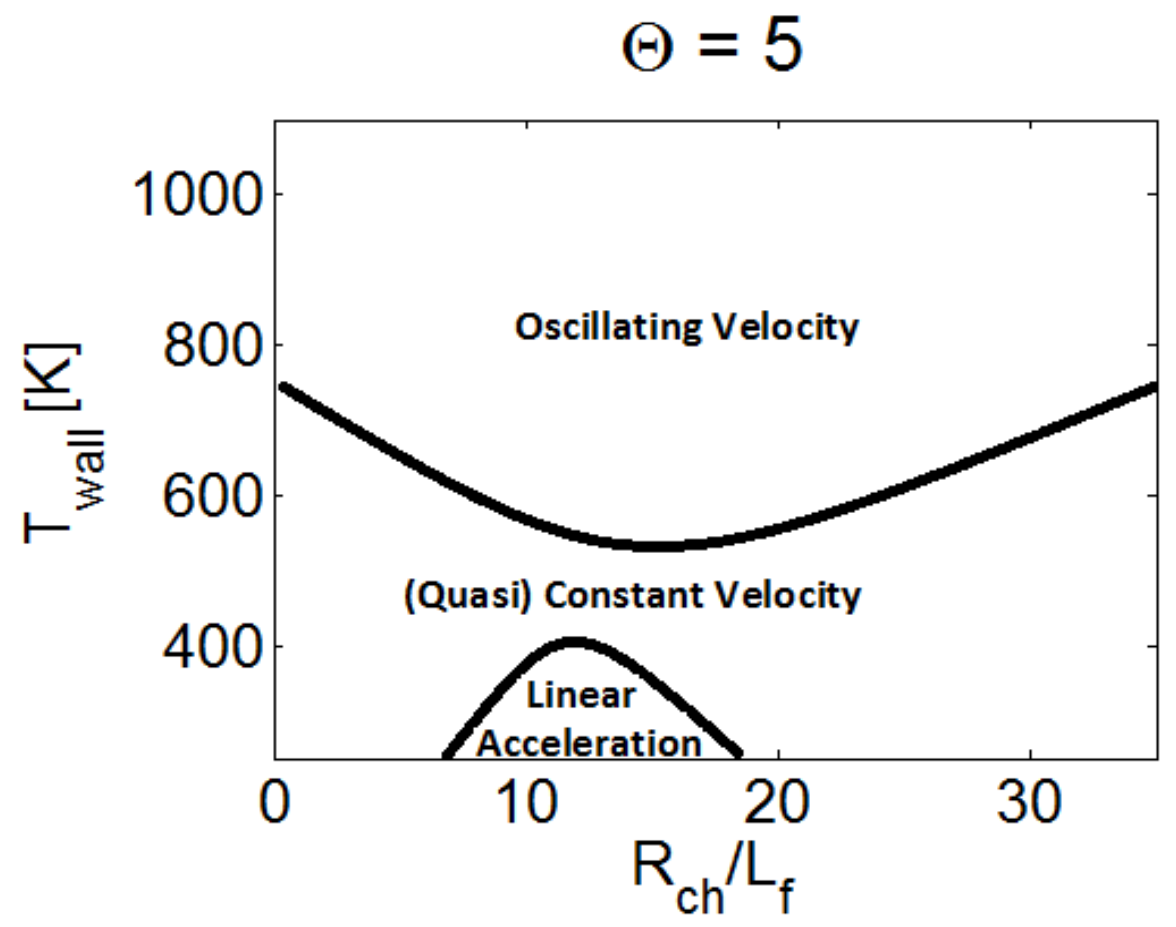

Figure 4.27. Flame propagation regimes as described by the channel wall temperature and width.

Three of these four regimes are shown in Figure 4.27 for $\Theta=5$. At the conditions shown therein, the flame extinction is not attained. This has been related to the way that the thermal expansion 
coefficient can control the heat lost at the flame front by setting the upper limit of the energy in the process (an example of this effect was shown in Figure 4.14). A linear acceleration regime is identified for the colder wall conditions and a certain channel width range. The faster flame propagation has been related to the larger flame stretching obtained in the presence of colder walls. When considering too narrow channels (e.g. $\mathrm{R}_{c h}<6 L_{f}$ ), this flame corrugation is less effective due to the proximity between the walls. In contrast, when the walls are too separated $\left(\mathrm{R}_{c h}>20 L_{f}\right)$, the overall thermal effect is weaker; therefore the linear acceleration region is also limited on the right as the channel width is further increased. As for the warmer walls, they moderate the flame propagation, principally by generating a more planar shape of the flame front. This effect can be observed in the central region, where either constant flame velocities or weak linear accelerations are obtained. A larger increase in the wall temperature leads the flame to propagate in an oscillating manner, which has been demonstrated to be a result of the better conditions that the flame finds to propagate near the wall, taking the flame tip away from the channel centerline. This oscillating velocity can reach very large values; but for a short time until the propagation near the wall is extended vertically to fill the channel width, reducing the flame surface area.

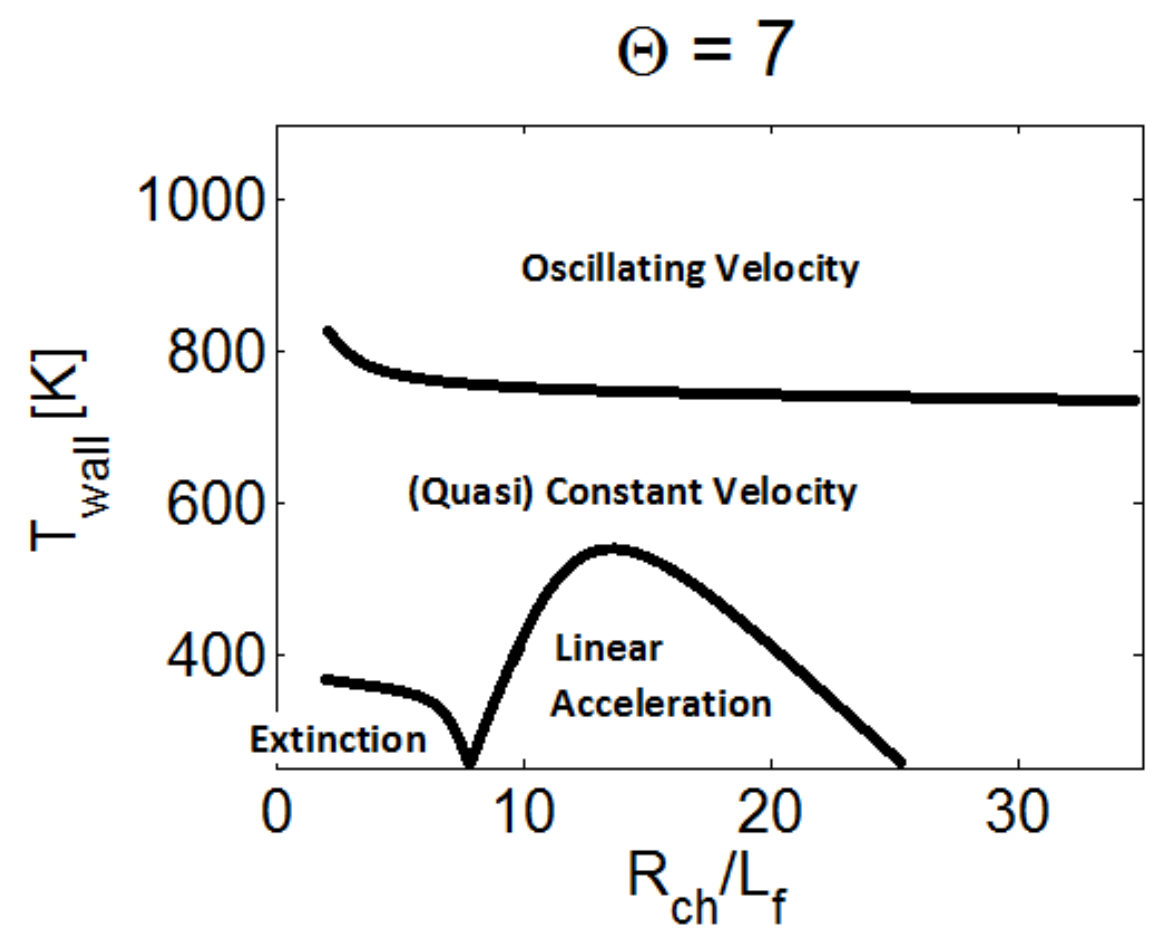

Figure 4.28. Flame propagation regimes as described by the channel wall temperature and width. 
Figure 4.28 is the counterpart of Figure 4.27 for a larger thermal expansion ratio, $\Theta=7$. In this case, the flame extinction is observed if cold enough wall conditions are employed in a channel narrower than a certain threshold width. The region where a linear flame acceleration occurs is observed near the extinction zone, in the vicinity of the width and temperature ranges described by $\Theta=5$. In this case, the region is extended toward larger widths as well as to warmer walls. By raising the energy released in the reaction zone, a larger heat loss is produced, which results in larger flame front corrugations at lower wall temperatures, and the fact that the thermal effect remains important for the combustion process even when the channel width is large. The region of weak linear acceleration and quasi-steady flame propagation is also observed to get widen when $\Theta=7$. A more steady form of the flame front is expected in a more energetic environment which results in the extension of this zone toward its upper limit. The oscillating character of the flame velocity is then restricted almost entirely to higher wall temperatures, independently of the channel width. Overall, a raise of the curves is obtained by increasing the thermal expansion ratio, which is consistent with the consequent raise of the thermal energy available in the system.

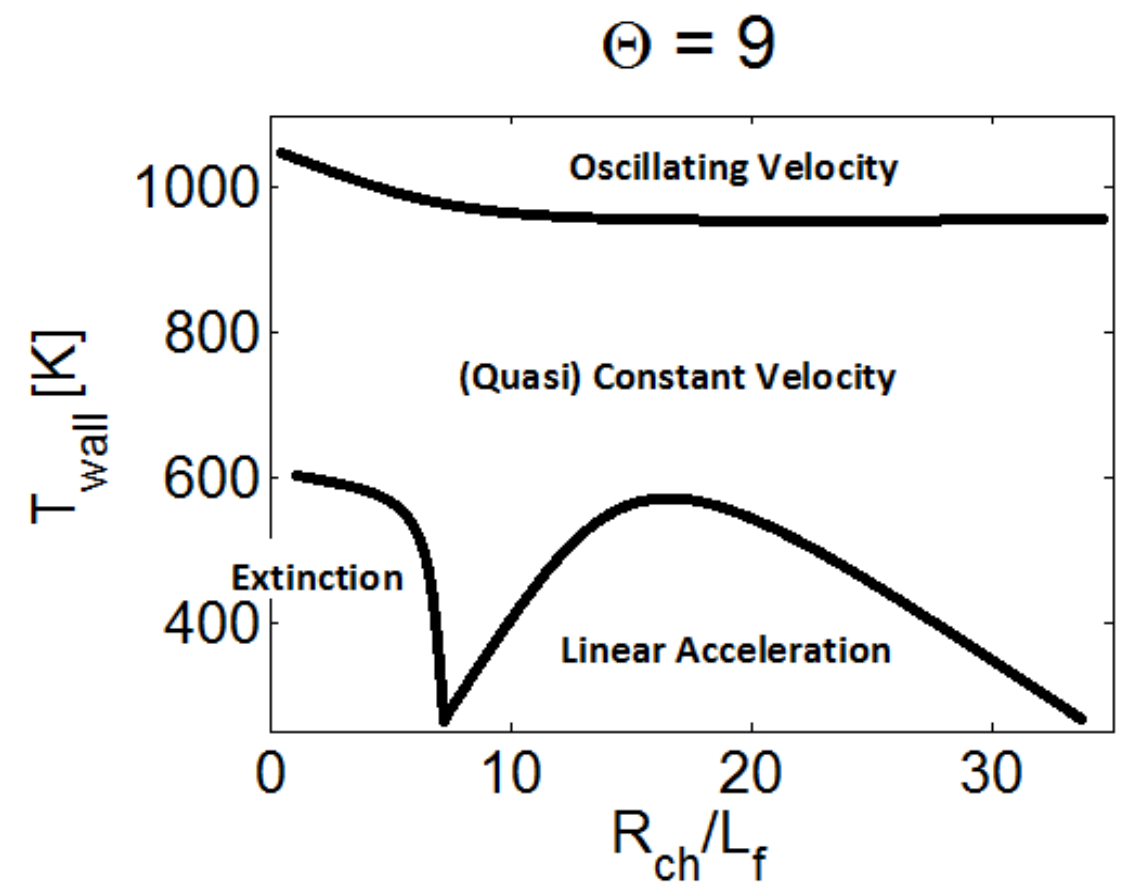

Figure 4.29. Flame propagation regimes as described by the channel wall temperature and width. The scenario related to a further increase of the thermal expansion coefficient, $\Theta=9$, is presented in Figure 4.29. This time, the flame extinction region is noticed to be considerably 
extended to higher wall temperatures, always in relation to small channel widths. The linear acceleration region is also extended to warmer wall temperatures and wider channels, and the oscillating behavior of the flame velocity is left to very high wall temperatures. The highly desirable controlled combustion process can be reached more easily as the fuel mixture is more energetic, namely, the changing flame tip location observed in high wall temperatures is mitigated by the larger energy released at the flame front. In addition, even faster flame velocities can be obtained in a wider range of conditions when a larger energy-release fuel mixture is in place.

It is also possible to study the wall thermal effect on the flame dynamics from the perspective of a fixed wall temperature. For example, in Figure 4.30, where a constant wall temperature of $300 \mathrm{~K}$ is considered, three regimes can be identified: (i) the flame extinction region related to the smaller channel widths; (ii) a linear flame acceleration zone, which extends to a large range of thermal expansion coefficients and channel widths; and (iii) slow flame propagation in the quasiconstant and constant flame velocity region, determined by the lower thermal expansion coefficients and narrower channels, and to increasing channel widths and thermal expansion values. In this wall temperature, no oscillating flame velocity regime is observed.

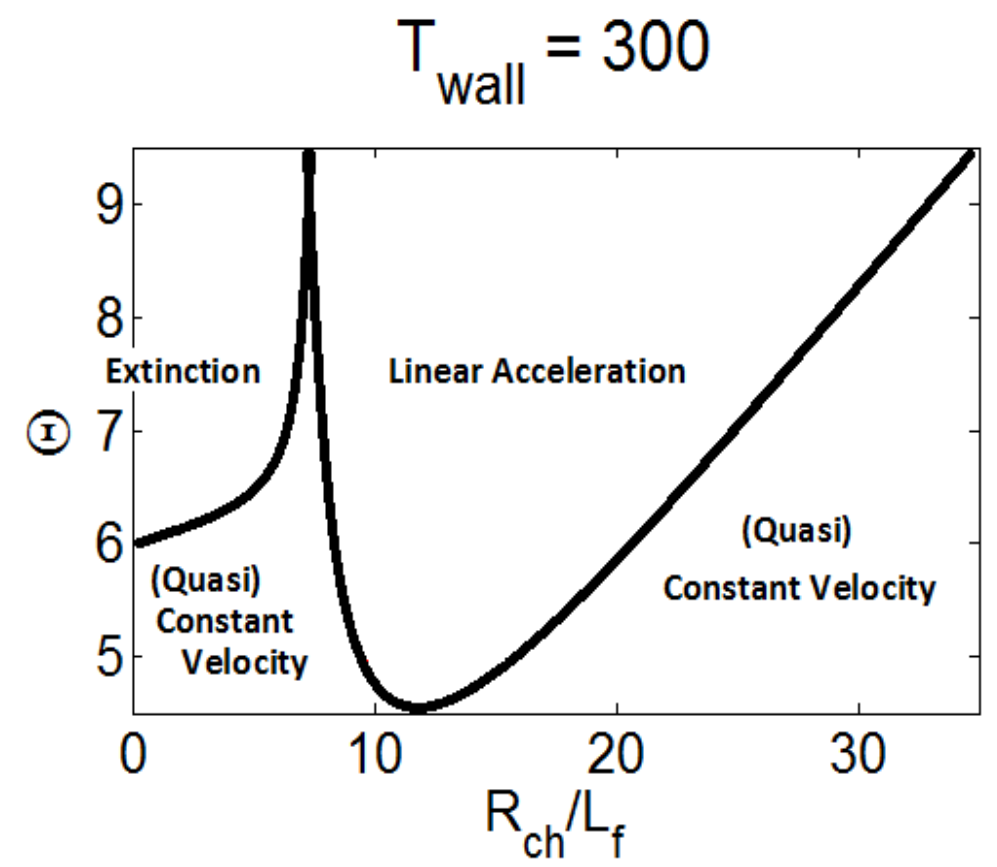

Figure 4.30. Flame propagation regimes described by the scaled width and thermal expansion coefficient. 


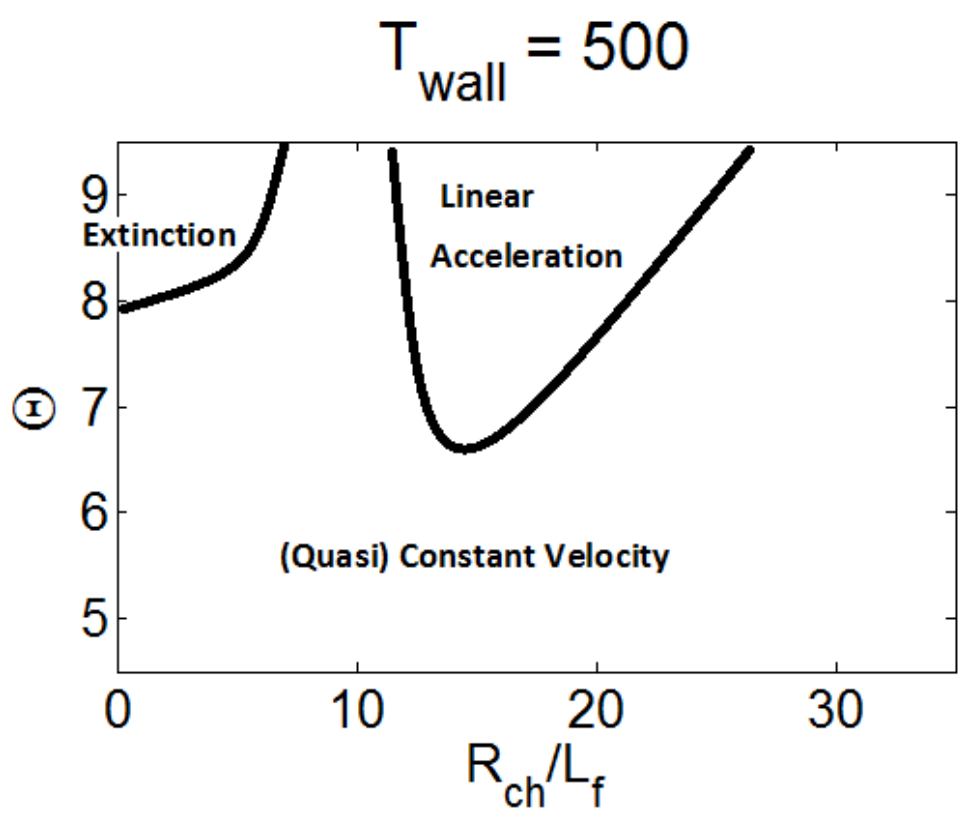

Figure 4.31. Flame propagation regimes described by the scaled width and thermal expansion.

In Figure 4.31, the attention is moved to the case where the channel walls are kept at $500 \mathrm{~K}$. The three regimes observed at $T_{w}=300 \mathrm{~K}$, Figure 4.30, are also seen here. However, the extinction and linear acceleration domains have been reduced. Faster flame propagation is limited to larger thermal expansion ratios and to a narrower range of channels. The flame propagation at this wall temperature occurs mainly at a constant speed or exhibiting a very weak acceleration. Again, there are no conditions for oscillating flame velocities in this case.

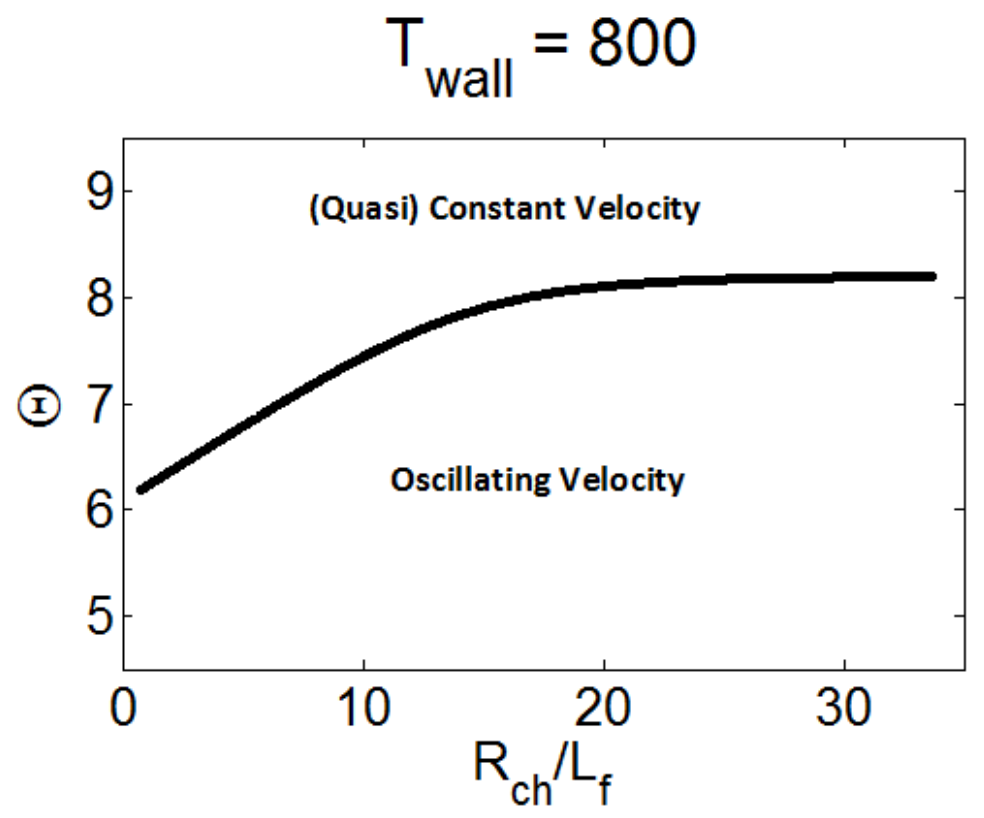

Figure 4.32. Flame propagation regimes described by the scaled width and thermal expansion. 
A further increase of the wall temperature modifies the regime diagram significantly. In Figure 4.32, where the wall temperature has been raised to $800 K$, only two regimes are observed: (i) the weak acceleration / constant propagation velocity regime and (ii) the oscillating velocity regime. In this case, a weakly accelerated or steady flame propagation is obtained in the narrower channels and/or at large enough thermal expansion ratios. The flame velocity has an oscillating character for most conditions, where large peak values can be obtained for short periods and at locations barely predictable. In contrast, linear flame velocities are not possible in this condition.

Finally, the regimes of flame propagation established by the fuel thermal expansion coefficient and channel width are identified in Figure 4.33, considering adiabatic wall conditions [63]. In the absence of heat transferred at the walls, the flame velocities are considerably larger, and the variation of the thermal expansions and channel widths does not establish four regimes, but only two; one given by an exponential acceleration of the flame velocity, and a second where this acceleration is not exponential, but much slower, i.e. linear.

\section{Adiabatic Walls}

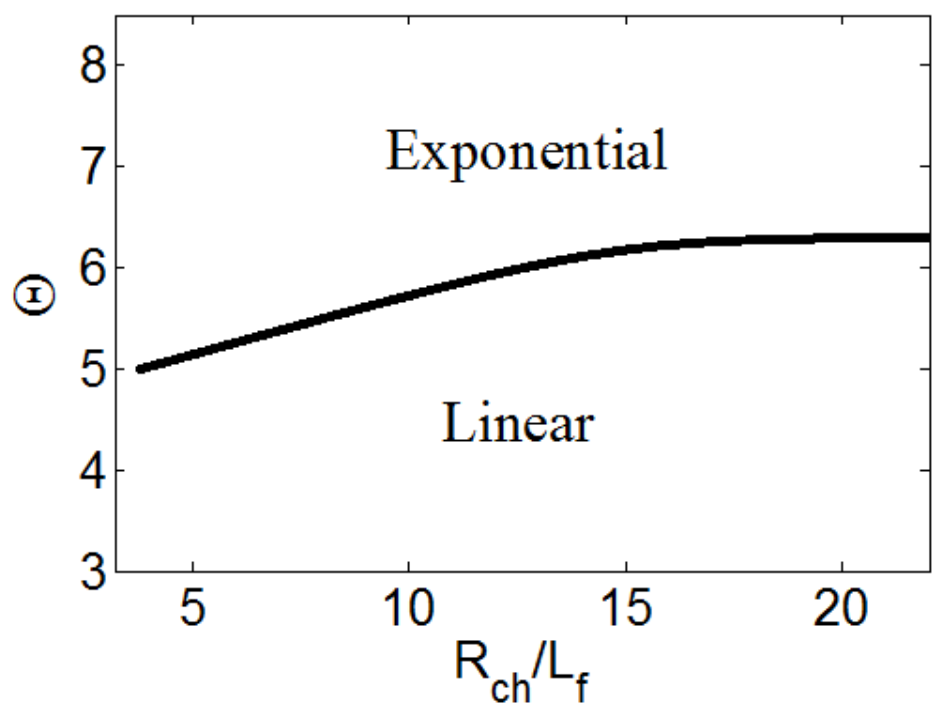

Figure 4.33. Flame propagation regimes in adiabatic conditions [63].

\subsubsection{Tubes versus Channels}

In addition to the 2D Cartesian version, the computational platform is also available for the cylindrical axisymmetric configuration. Therefore, a comparison of the flame dynamics trends observed in 2D channels and cylindrical tubes has also been performed, and the results are presented in Figure 4.34 and 4.35. Figure 4.34 shows the flame propagation velocities attained 
in isothermal $T_{w}=400 \mathrm{~K}$ and adiabatic tubes and channels. The large difference in the flame propagation at adiabatic conditions versus the controlled by isothermal walls previously seen in channels, is also noticed in tubes. Moreover, a slower flame propagation is seen for tubes in the beginning of the process, which later collapses to the velocity observed in the channel case.

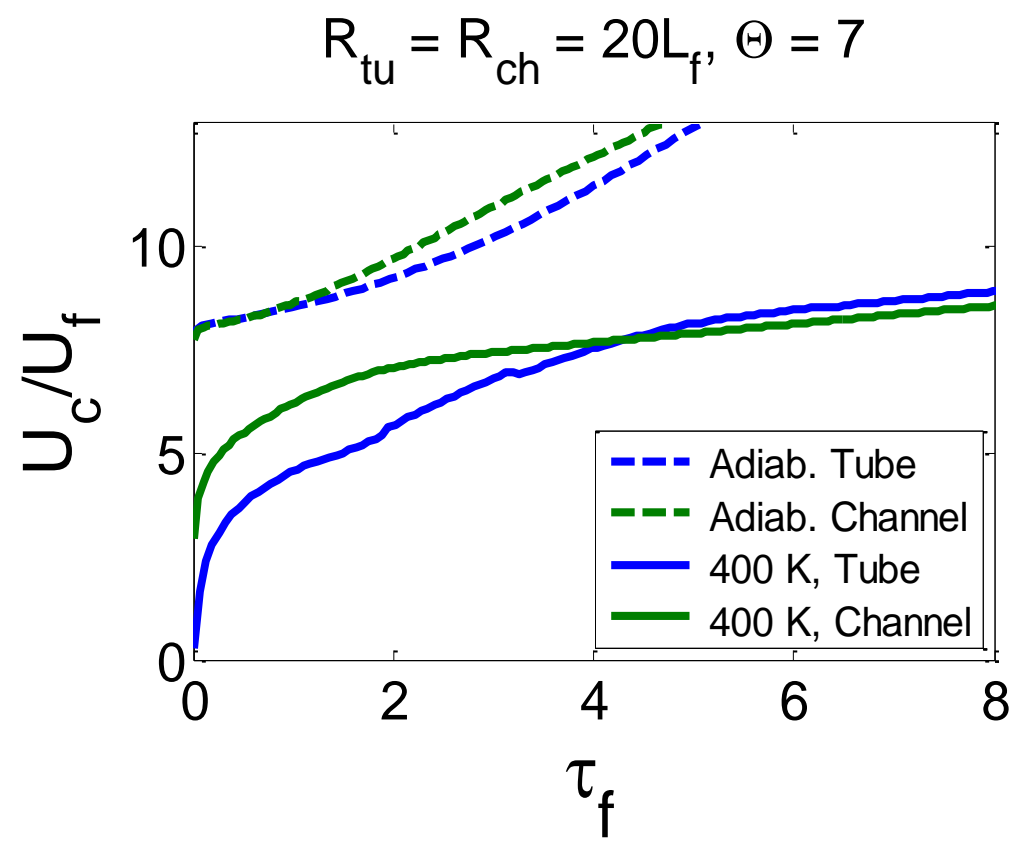

Figure 4.34. Flame velocities in tubes and channels, with adiabatic and isothermal conditions.

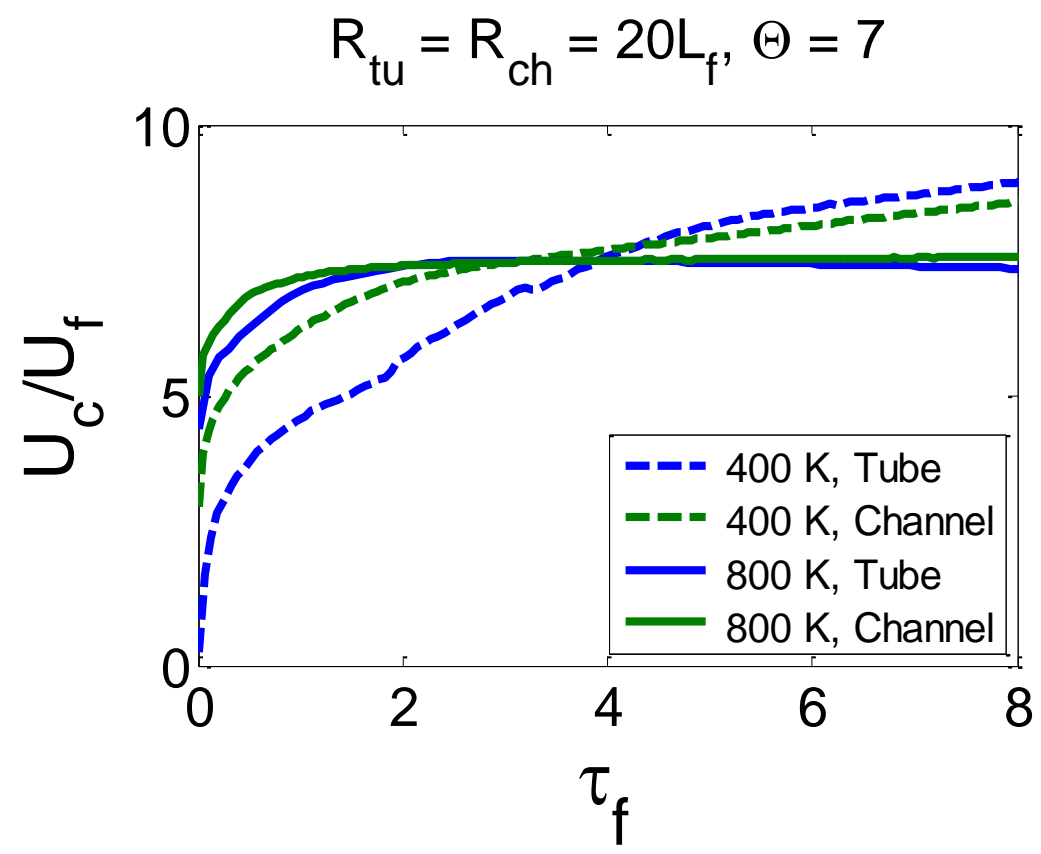

Figure 4.35. Flame velocities in tubes and channels with two different isothermal conditions. 
In Figure 4.35, faster flame propagation enhanced by colder wall conditions is observed in isothermal tubes as well. Cylindrical tubes promote the flame propagation because of the larger flame front surface approximated by including another dimension. This is reflected in the $T_{W}=$ $400 \mathrm{~K}$ case, where the tube configuration determines a steeper linear velocity trend than the channel one. Moreover, the propagation rate does not exhibit much difference when raising the wall temperature $\left(T_{w}=800 \mathrm{~K}\right)$ on the two conduits, being reduced to a constant speed.

\subsubsection{Later Stages}

The flame propagation in isothermal pipes experiences a significant change in later stages, as seen in Figure 4.36, where the combustion process occurring in a channel $\mathrm{R}_{c h}=10 L_{f}$ and walls kept at $T_{w}=300 \mathrm{~K}$ is shown, along with the adiabatic case. Here, the flame velocity decays drastically as a result of the cooling of the burned gas, which can even produce the backwards displacement of the flame front region (Figure 4.37). Such a displacement is produced as a result of the contraction that burned gas volume undergoes as it gets colder.

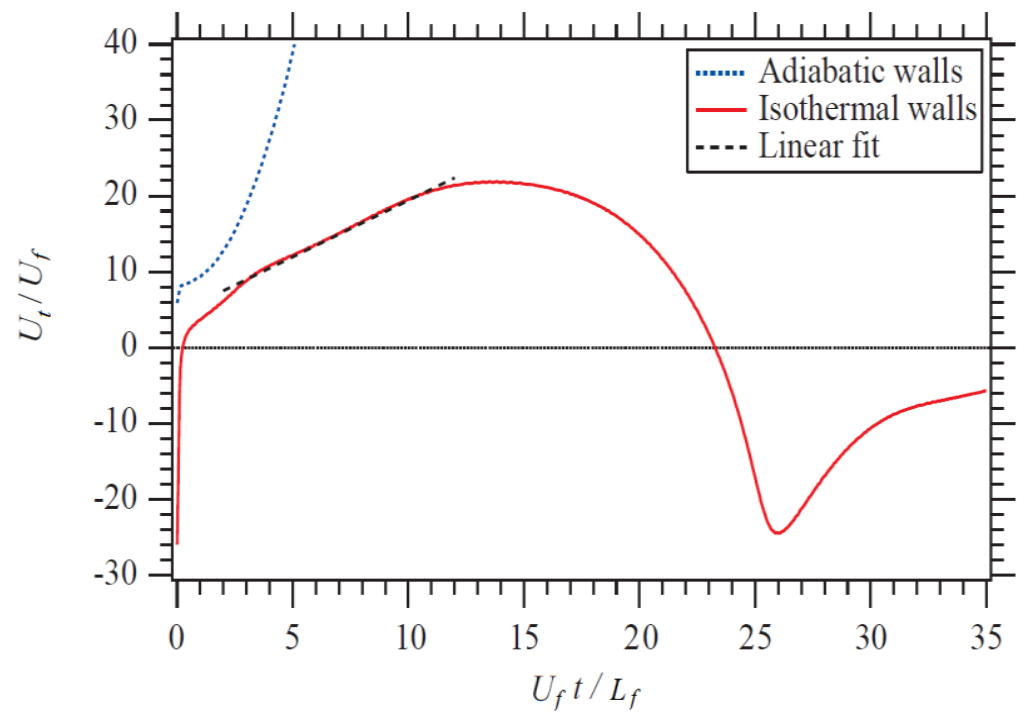

Figure 4.36. Flame velocities in channels at later stages, $\Theta=8, \mathrm{R}_{c h}=10 L_{f}, \mathrm{~T}_{W}=300 \mathrm{~K}[64]$.

In Figure 4.36, the linear stage corresponding to this wall temperature condition is fitted by a dashed line, and it is also observed how the flame deceleration is followed by a time interval where the flame velocity becomes negative, which represent the displacement of the region towards the pipe closed end. Therefore, it can be inferred that the flame propagation produced in pipes subjected to isothermal conditions does not experience a transition to detonation. The flame does not accelerate enough to produce strong shock waves that can lead to DDT, but the 
faster velocities observed in colder pipes are replaced by a deceleration and eventual extinction. The same conclusion can be expected when hotter walls are in place, since the flame velocities associated to these conditions are slower, although the extinction process may take longer.

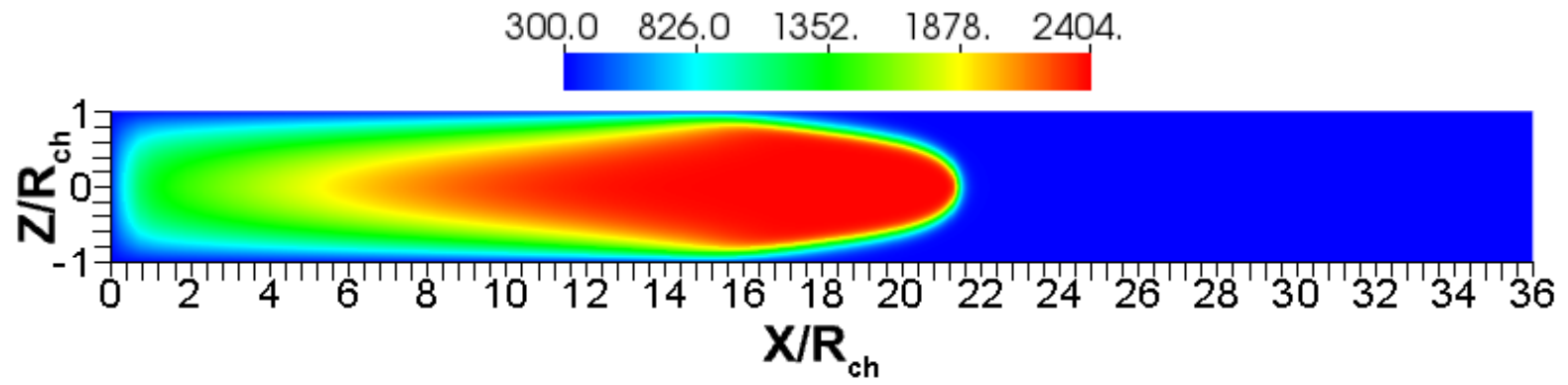

a)

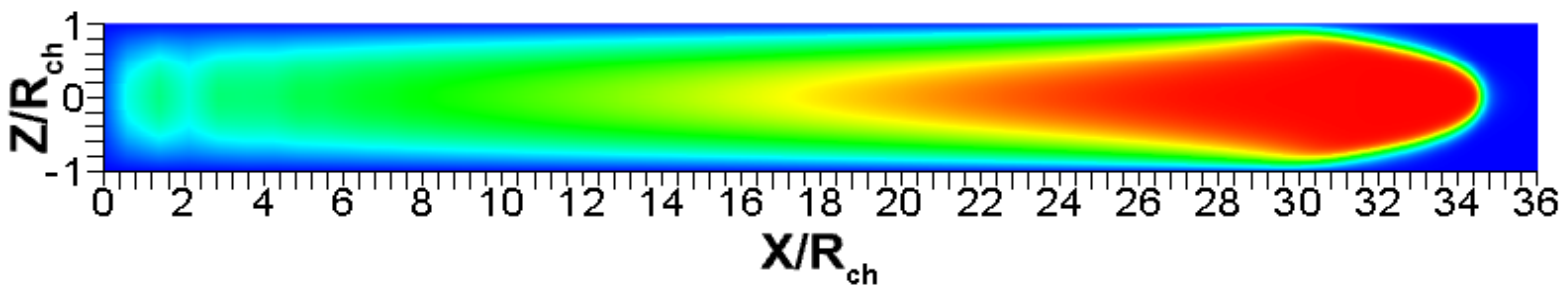

b)

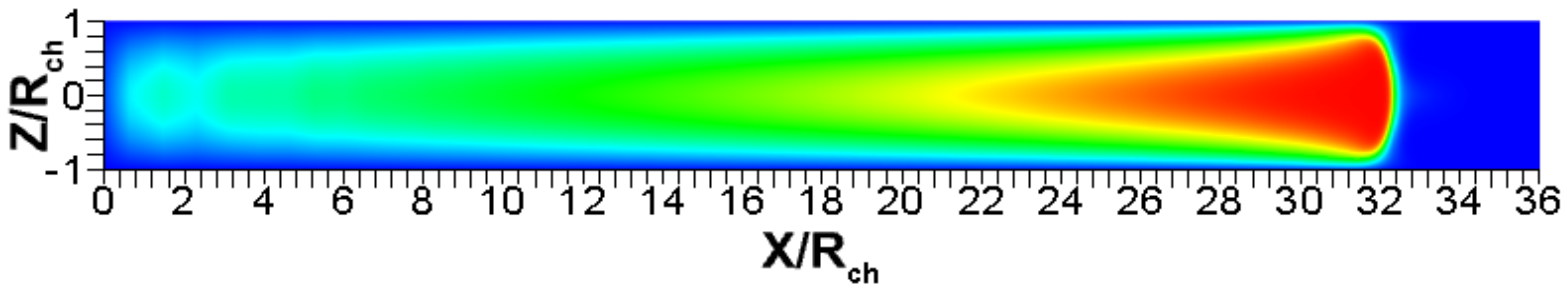

c)

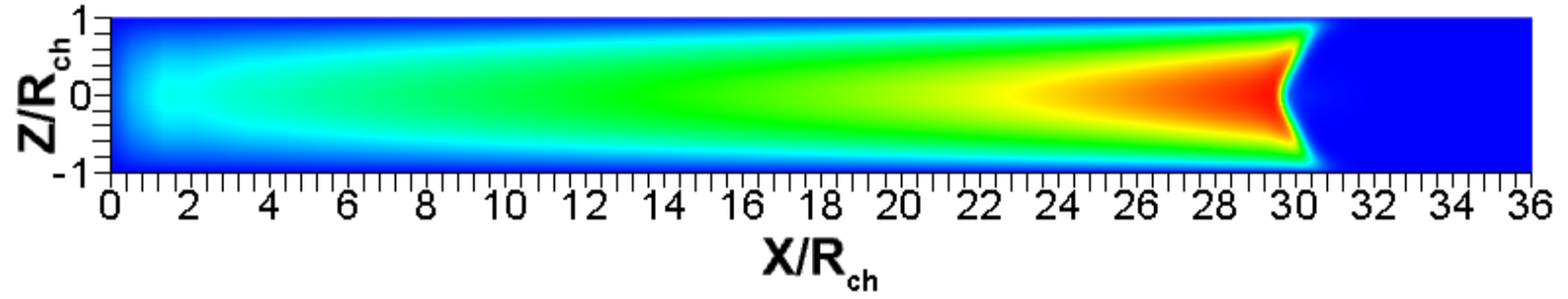

d)

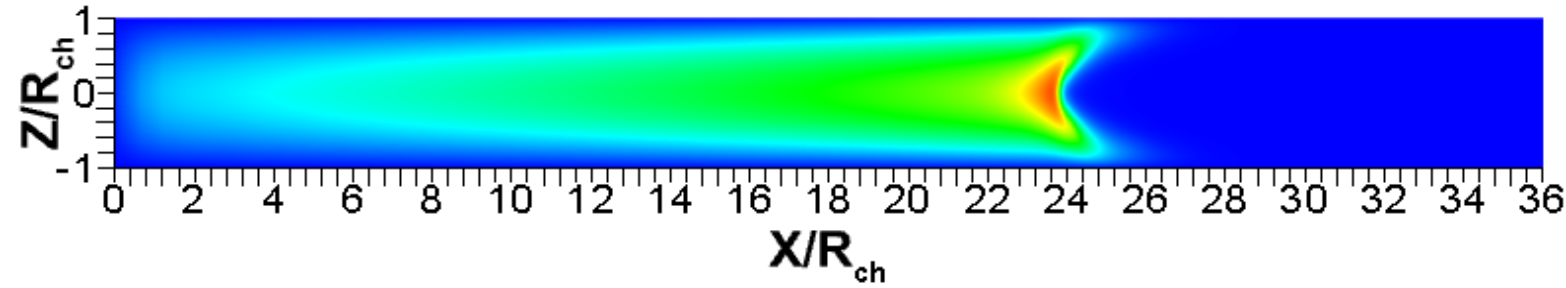

e)

Figure 4.37. Flame propagation including later stages. $\mathrm{T}_{W}=300 \mathrm{~K}, \Theta=8, \mathrm{R}_{c h}=20 L_{f}$. a) $\tau_{f}=$ 14.5, b) $\tau_{f}=22.8$, c) $\tau_{f}=25.4$, d) $\tau_{f}=26.6$, e) $\tau_{f}=30.4$. 


\subsection{Flame Dynamics in Obstructed Channels}

In this part of the investigation, the channel has been modified to include obstacles at the walls. Such a structural change of the combustor has the capability to generate much faster flame propagations [39], as a result of the larger stretching attained by the flame surface, and even the possibility of turbulent combustion regimes. Among the different configurations of obstacles that could be included in the channel, the present research is focused on the so-called 'tooth-brush' configuration, which was originally proposed by Bychkov et al. [41] considering adiabatic conditions. The scenario is illustrated in Figure 4.38, where isothermal surfaces are included.

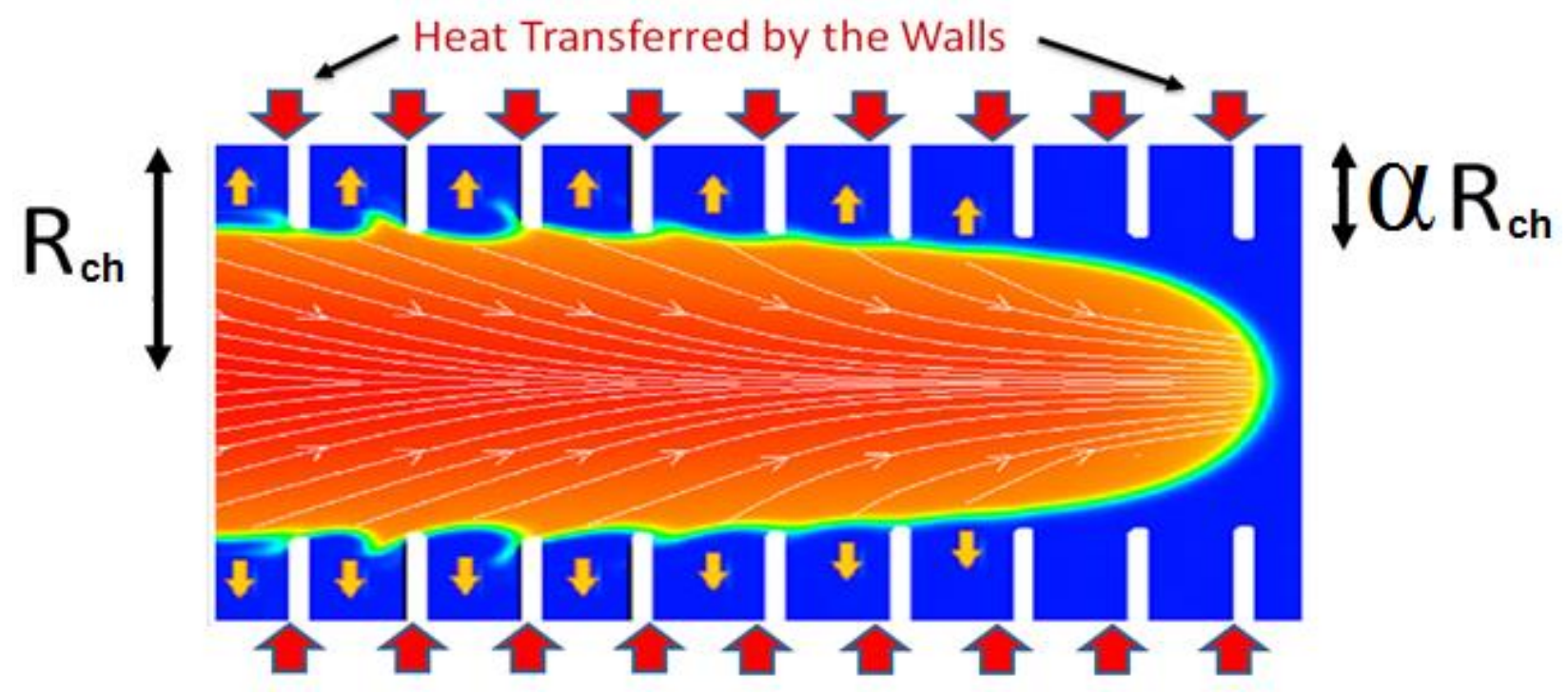

Figure 4.38. Description of the flame propagation in a channel of width $2 R_{c h}$, where obstacles of length $\alpha R_{c h}$ have been included, considering isothermal wall conditions.

In this configuration, the flame propagation is also enhanced by the expansion of the burned gas in the regions between obstacles or 'pockets.' As the burning of the fuel mixture in the pockets is completed, the flame propagating along the channel has already moved downstream. The volumetric expansion of the burned gas in the pockets results in a delayed force enhancing the flame propagation, which added to the expansion of the burned gas in the center of the channel, produces an intense jet flow in the channel core. If the channel walls are isothermal, the heat exchange at the walls modifies the temperature of the fuel mixture in the pockets and, consequently, the volumetric expansion of the burned gas in it. 
In principle, the intense jet propagation generated in this tooth-brush configuration is related mainly to a laminar combustion regime. The obstacles are placed very close to each other, making the formation of eddies in the center of the channel difficult. The flame velocities obtained in this configuration depend on structural parameters such as the channel width, obstacle size, and the distance between obstacles, as well as on the thermal conditions at the walls. In this section, the intensity of the flame propagation is investigated in relation to these parameters. First of all, the flame velocities are investigated in relation to the size of the obstacle, since it is this variable which makes the configuration distinctive from the 'smooth' wall scenario studied in Section 4.1.

\subsubsection{Obstacles Size Effect on the Flame Propagation}

The obstacles size is characterized by the blockage ratio $\alpha(0<\alpha<1)$, which relates the length of the obstacles to the channel width as $l_{o b s}=\alpha R_{c h}$, where $R_{c h}$ is half the width of the channel. This parameter influences the flame dynamics basically in two manners: (i) it identifies (along with the distance between obstacles) the region where the delayed burning occurs, and (ii) it determines the channel unobstructed section, which modifies the elongation of the flame surface as it propagates along the channel centerline.

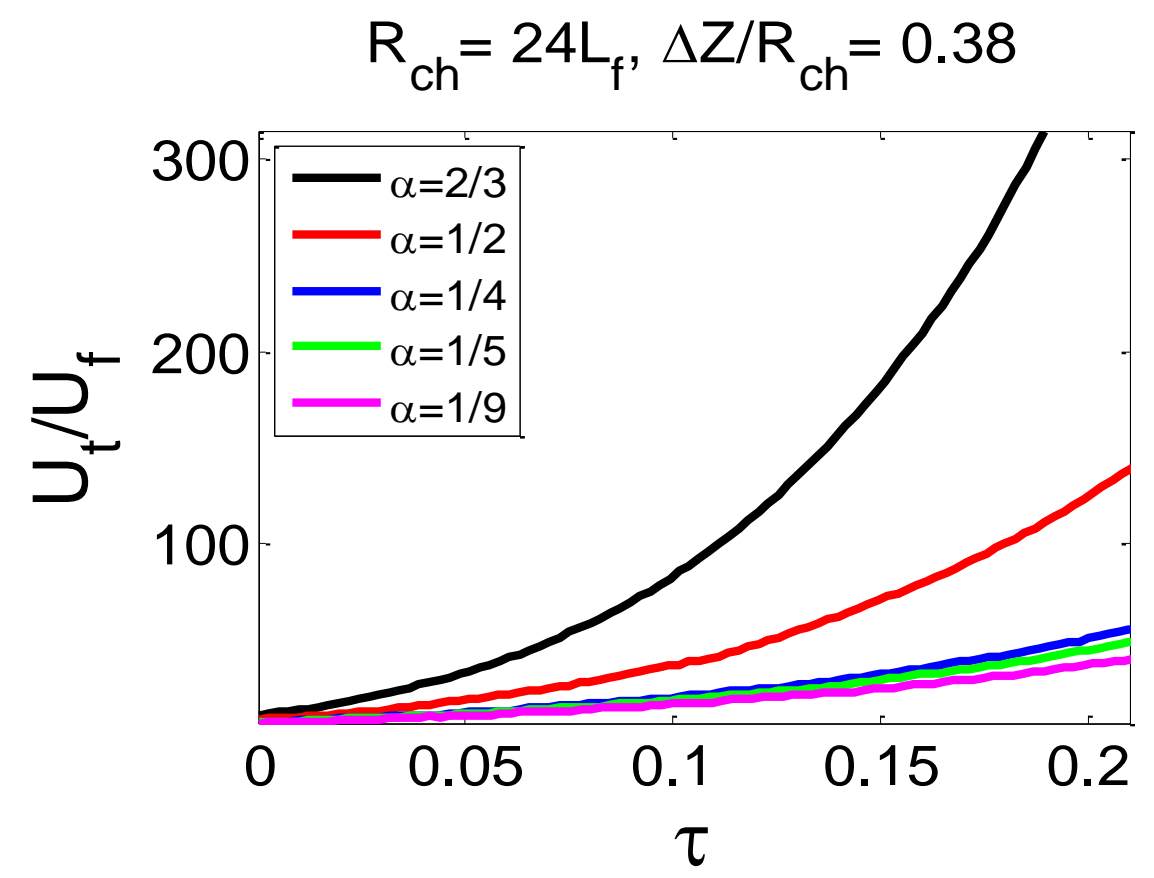

Figure 4.39. Flame velocities obtained in obstructed channels with different blockage ratios. 
Figure 4.39 shows the scaled flame propagation velocity versus the scaled time $\tau=t U_{f} / R_{c h}$, observed in obstructed channels at various blockage ratios $\alpha$. It is shown that the flame propagation varies significantly with $\alpha$, and more clearly within a certain range. Specifically, while the flame velocity does not change much as the obstructed section is raised from $11 \%$ to $25 \%$, (blue, $\alpha=1 / 4$, green, $\alpha=1 / 5$, and magenta, $\alpha=1 / 9$ lines), a significant acceleration is observed when the obstacles occupy more than $50 \%$ of the channel section $(\alpha=1 / 2$ and $\alpha=$ $2 / 3$ ). It is nevertheless noted that the flame velocity trends exhibit an exponential behavior versus time even when smaller obstacle sizes are in place.

The tendency is similar when channels of different widths are considered. Figures 4.40 and 4.41 compare the flame velocities in channels with different widths $\left(R_{c h}=20 L_{f}\right.$ in Figure 4.40 and $R_{c h}=30 L_{f}$ in Figure 4.41), and various blockage ratios. In these cases, in addition to the scenarios given by $\alpha=1 / 5$ and $\alpha=1 / 2$, much smaller blockage ratios have been included; namely, $\alpha=1 / 20$ and $\alpha=1 / 15$. The flame velocities associated to the smaller obstacles does not differ much from, for example, the $20 \%$ obstruction case provided by $\alpha=1 / 5$. Therefore, it can be concluded that the flame velocity is enhanced by the obstacles, but a more efficient enhancement is observed when long enough obstacles are in place.

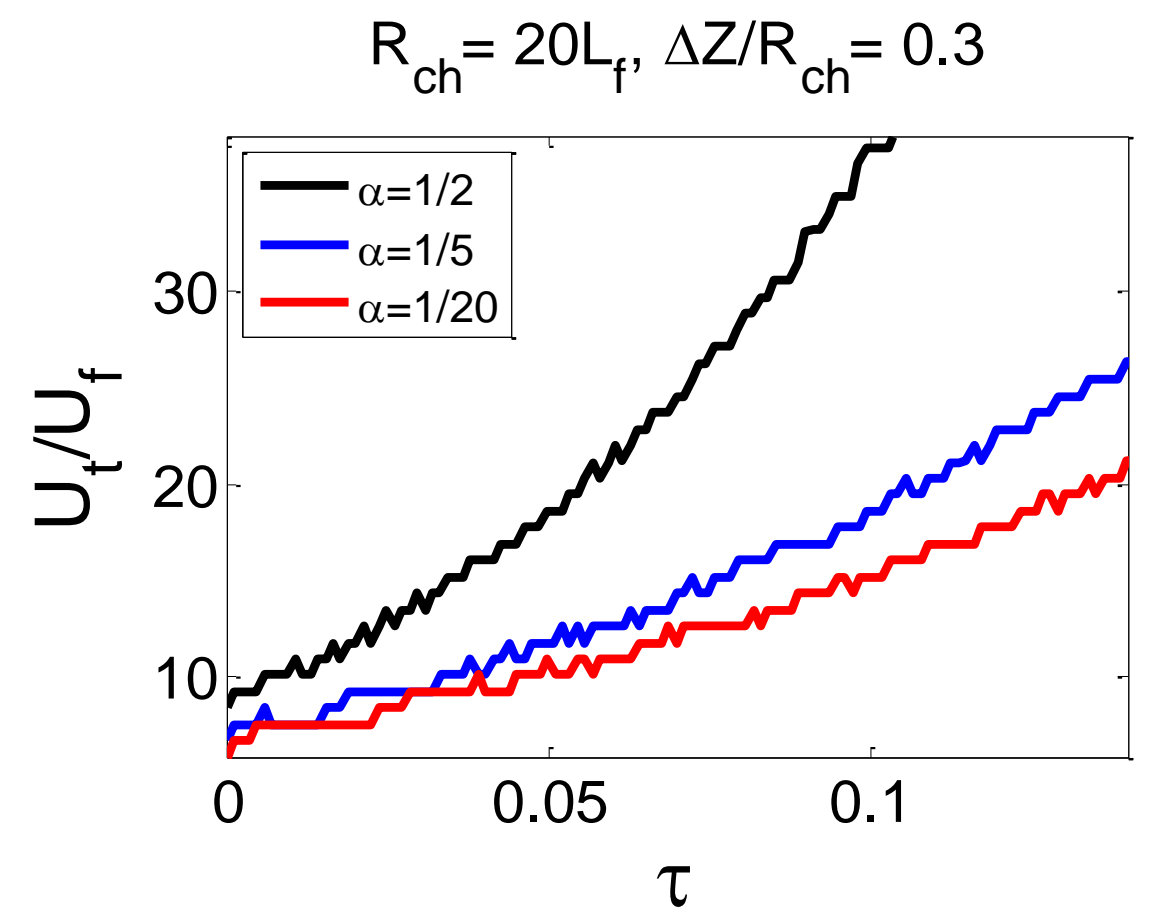

Figure 4.40. Flame tip velocities obtained in obstructed channels with different $\alpha, R_{c h}=20 L_{f}$. 


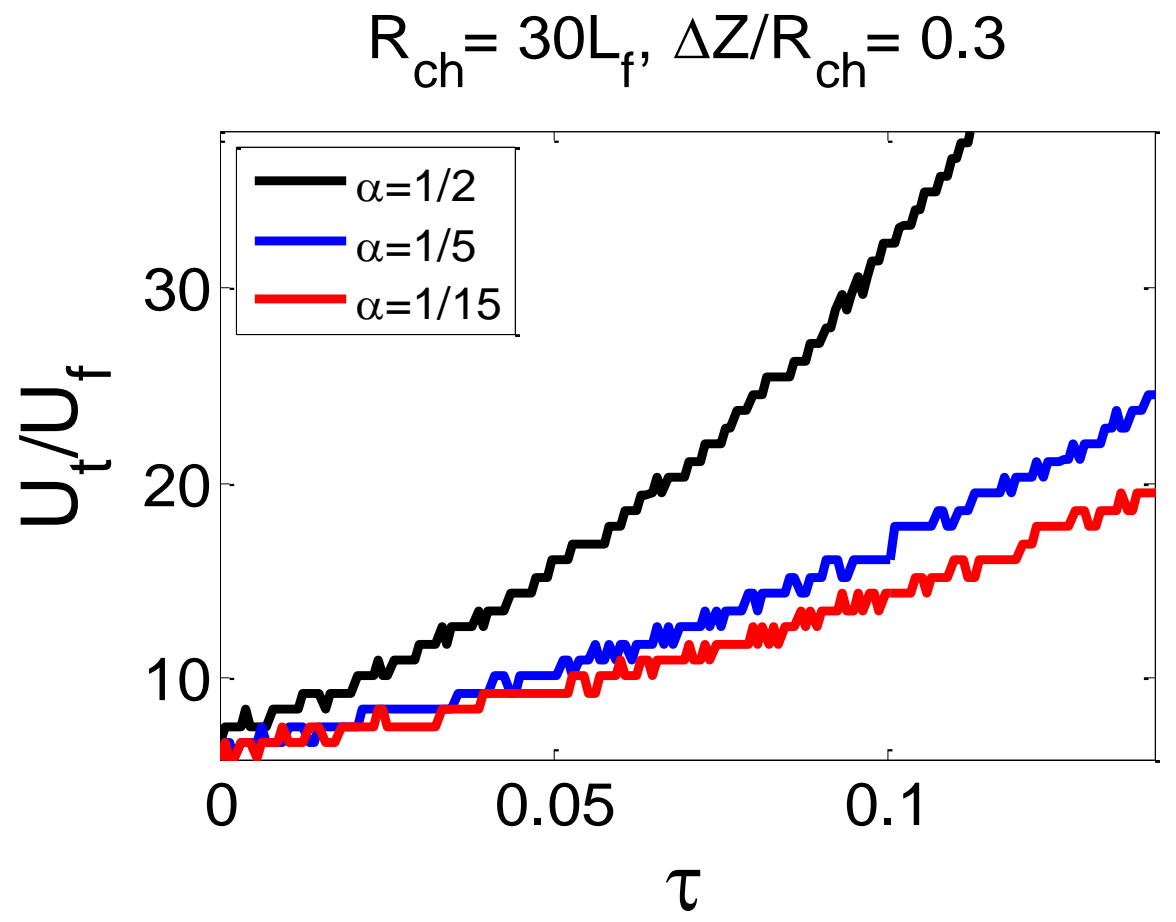

Figure 4.41. Flame tip velocities obtained in obstructed channels with different $\alpha, R_{c h}=30 L_{f}$.

An interesting result observed in Figure 4.40 and 4.41 is that the flame velocities do not change significantly when increasing the channel width. This observation is extended in the next section, where some qualitative differences from the 'smooth' channel case are started to be noticed.

\subsubsection{Effect of the Channel Width}

When 'smooth' unobstructed conditions are considered at the channel walls, the flame dynamics exhibit a strong dependence on the channel width. This is related to the fact that it is the pipe width that determines how big the boundary layer thickness produced by the nonslip conditions is as compared to the flame front surface, and how far the hotter regions of the burned gas/flame front is from the walls. However, when obstacles are included into the system, the story seems to be different. Figure 4.42 presents the time evolution of the flame propagation observed in channels of different widths. The flame tip position along the centerline of the pipe has a similar trend as the channel width is reduced from $60 L_{f}$ to $40 L_{f}$, considering either a $20 \%$ or $50 \%$ blockage ratio $(\alpha=1 / 5$ and $\alpha=1 / 2)$. 


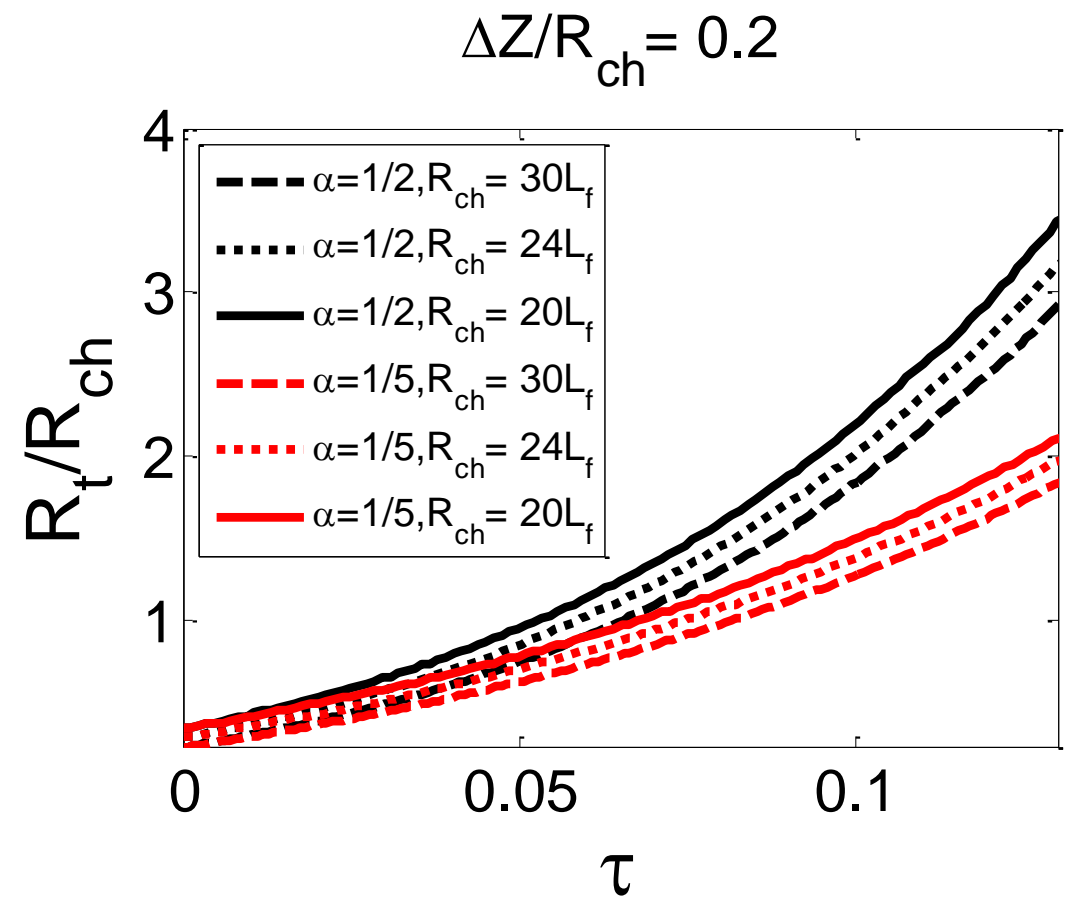

Figure 4.42. Evolution of the flame tip position in a channel considering different widths.

Since the flame propagating along the axis is kept out of the walls by the obstacles, this result is not necessarily surprising. Indeed, the flame propagation occurs through the free part of the channel section, therefore, narrower channels produce faster propagations due to the larger stretching of the flame surface. However, such a propagation enhancing is mitigated by the fact that the pocket region is characterized as a fraction of the channel width $\left(\alpha R_{c h}\right)$, determining smaller pockets for narrower channels. This generates a weaker contribution of the delayed volumetric expansion in the pockets to the flame propagation when $R_{c h}$ is smaller. The proportional relation between the channel width and the obstacle size leads to the conclusion that it is possible to extrapolate the flame propagation characteristics observed for a certain $R_{c h}$ value, to a different channel width, if the blockage ratio is the same.

These cases are related to obstacles closely placed at the walls, with the spacing between them varying from $10 \%$ to $20 \%$ of the channel width $\left(\Delta Z / R_{c h}=0.2-0.38\right)$. The 'tooth-brush' configuration was suggested to promote quasi-laminar regimes on the flame propagation due to the proximity between obstacles. In the next section, the separation is increased, and the effect of this extension is evaluated in relation to the attained flame velocities and burning regimes. 


\subsubsection{Obstacle Separation Effect}

The spacing between neighboring obstacles is another important parameter in obstructed pipes. Along with the blockage ratio, it identifies the pocket volume where the delay burning occurs. In addition, this parameter modifies the vertical flame propagation occurring in the pockets, along the obstacle surface. Figures $4.43,4.44$ and 4.45 show the flame propagation velocities when multiple obstacles spacing are considered, $\Delta Z / R_{c h}=0.2 ; 0.6$ and 1 . These figures, in turn, include different blockage ratios, namely, $\alpha=1 / 5$ in Figure 4.43, $\alpha=1 / 2$ in Figure 4.44 and $\alpha=2 / 3$ in Figure 4.45, keeping the channel width constant for all the cases.

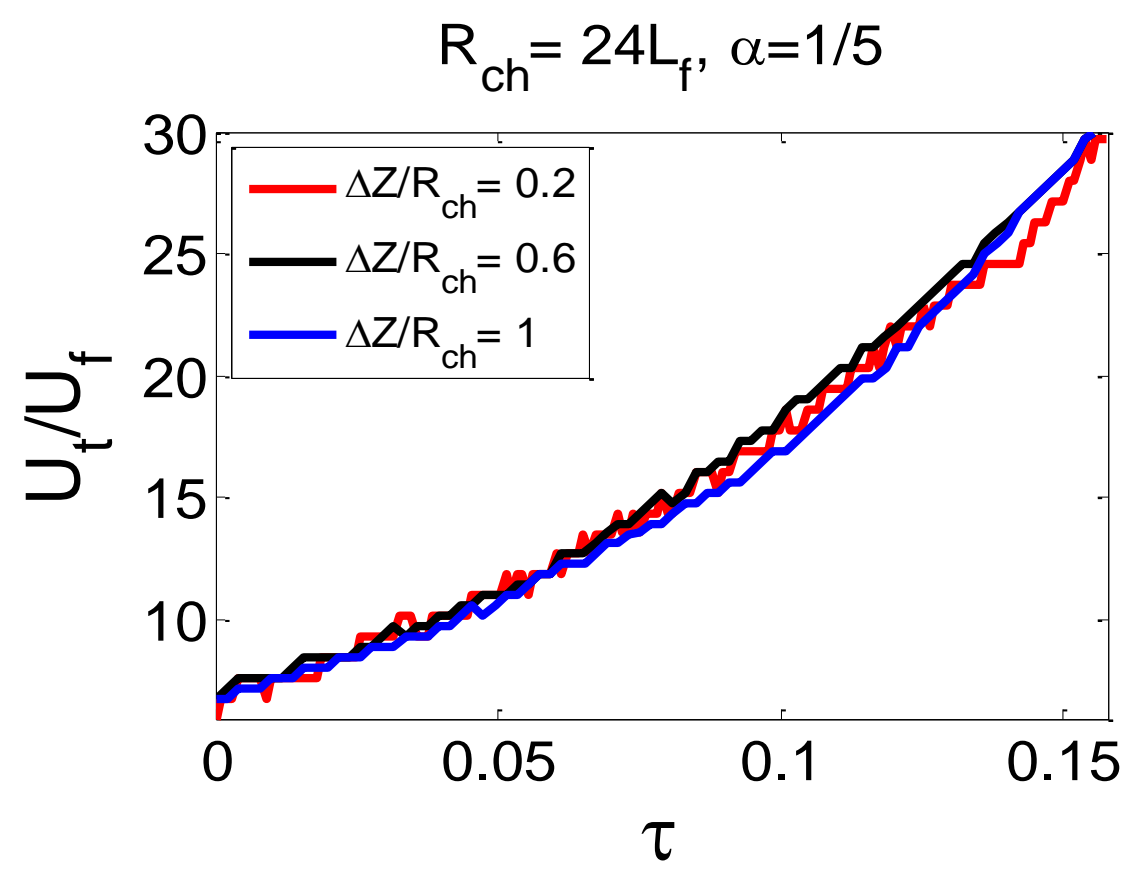

Figure 4.43. Flame velocities in obstructed channels, considering different obstacle separations.

Figure 4.43 shows not significant difference in the attained flame velocities. All three curves demonstrate an exponential acceleration, with a smoother behavior as the spacing gets bigger, that can be related to the lower frequency at which the flame 'finds' an obstacle. Only a minor transition is noticed for the larger separation case $\left(\Delta Z / R_{c h}=1\right.$, blue line), which initially provides a slower flame propagation, but ends up giving a slightly faster one. In Figure 4.44, this transition becomes more evident, as the blockage ratio is increased from $\alpha=1 / 5$ to $\alpha=1 / 2$. In this case, the velocity trend when $\Delta Z / R_{c h}=1$ has an oscillating behavior, accelerating with respect to the smaller obstacles separation cases. When the obstacle size is further increased (Figure 4.45), it is not only the larger spacing that shows an oscillating trend but the intermediate 
spacing size too $\left(\Delta Z / R_{c h}=0.6\right.$, red line $)$, which seems to indicate a transition to a more unstable flame propagation regime.

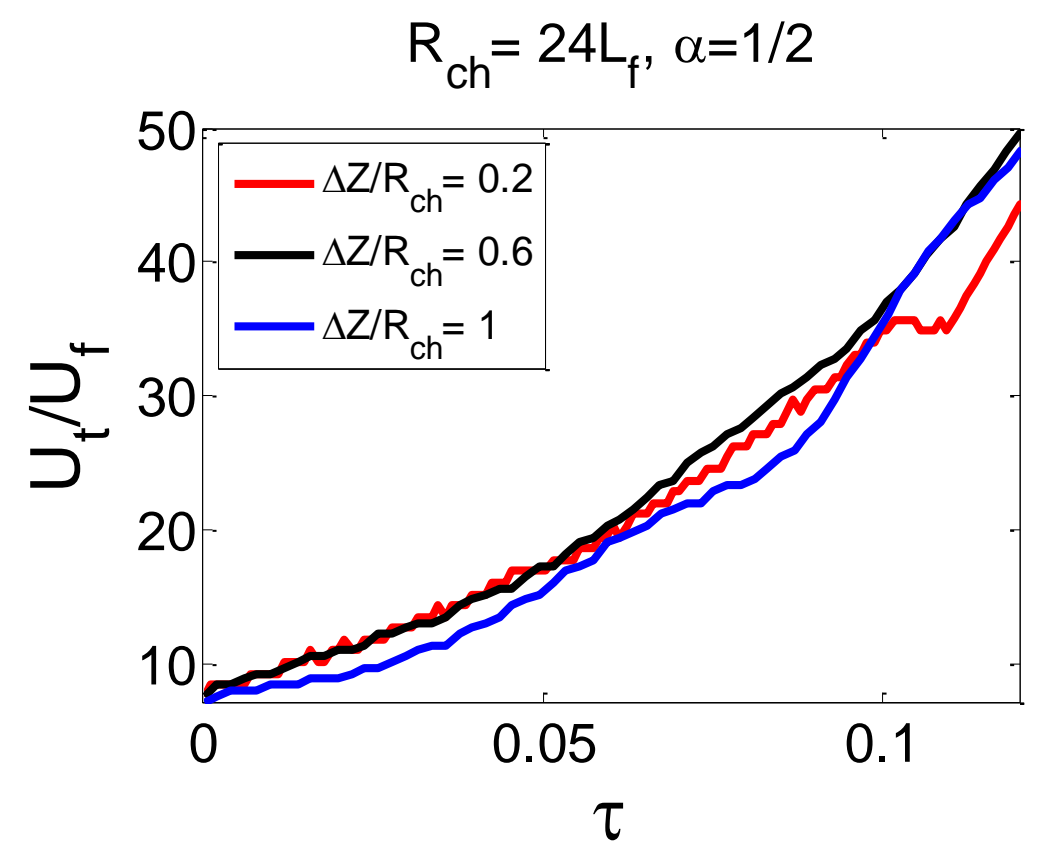

Figure 4.44. Flame velocities in obstructed channels, considering different obstacle separations.

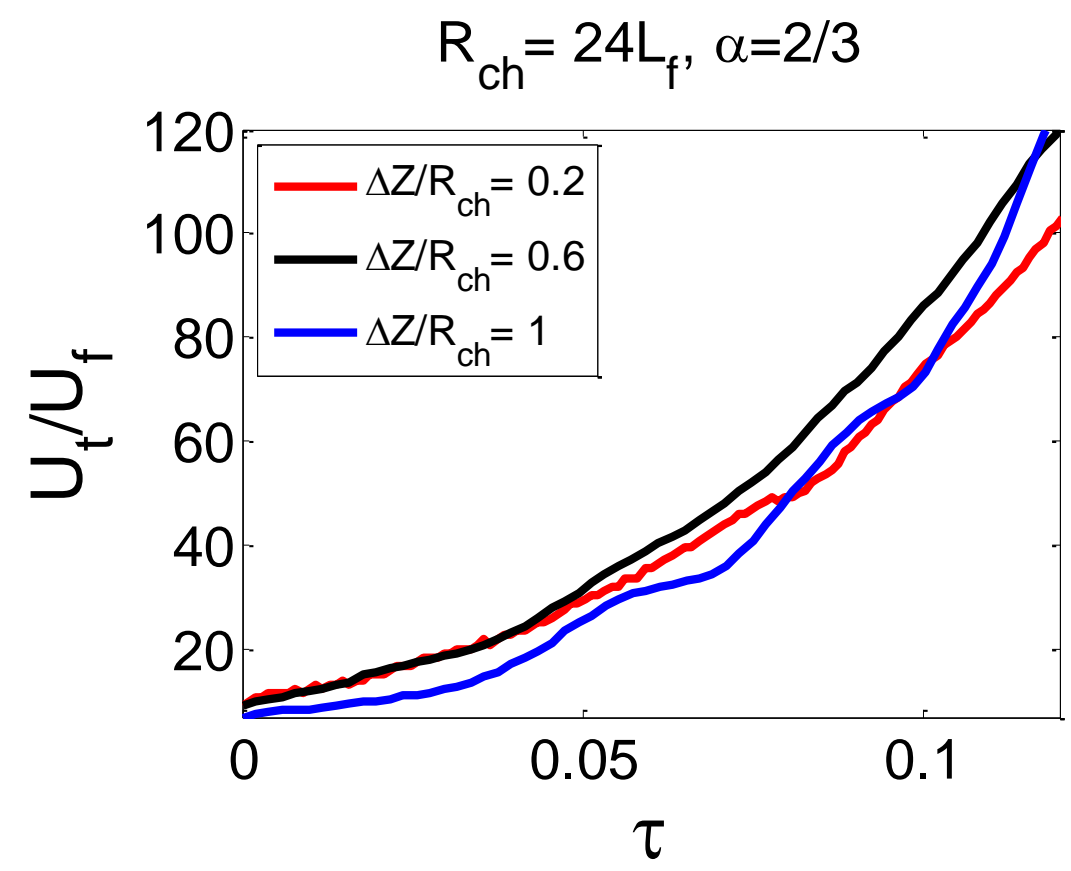

Figure 4.45. Flame velocities in obstructed channels, considering different obstacle separations. These observations are extended in Figure 4.46, by looking at the temperature distribution of the flames propagating in channels with three obstacle separations, and same blockage ratio $(\alpha=$ $2 / 3$ ). Figure 4.46 a includes the obstacle spacing $\Delta Z / R_{c h}=0.38$, where a laminar propagation is 
observed in the horizontal direction, with the flame distorting vertically as it 'hits' the obstacles. By raising the separation between obstacles to $\Delta Z / R_{c h}=0.6$ (Figure $4.46 \mathrm{~b}$ ), the flame shape becomes distorted along the channel center, and the erratic behavior is stressed in the vertical direction. In Figure $4.46 \mathrm{c}$, the separation between obstacles is increased to $\Delta Z / R_{c h}=1$, and the propagation becomes turbulent, with the flame front further distorted and discontinued. This transition explains the oscillating flame velocity observed in Figure 4.45 as the obstacles spacing increases, which results from the larger flame surfaces attained in turbulent combustion regimes.

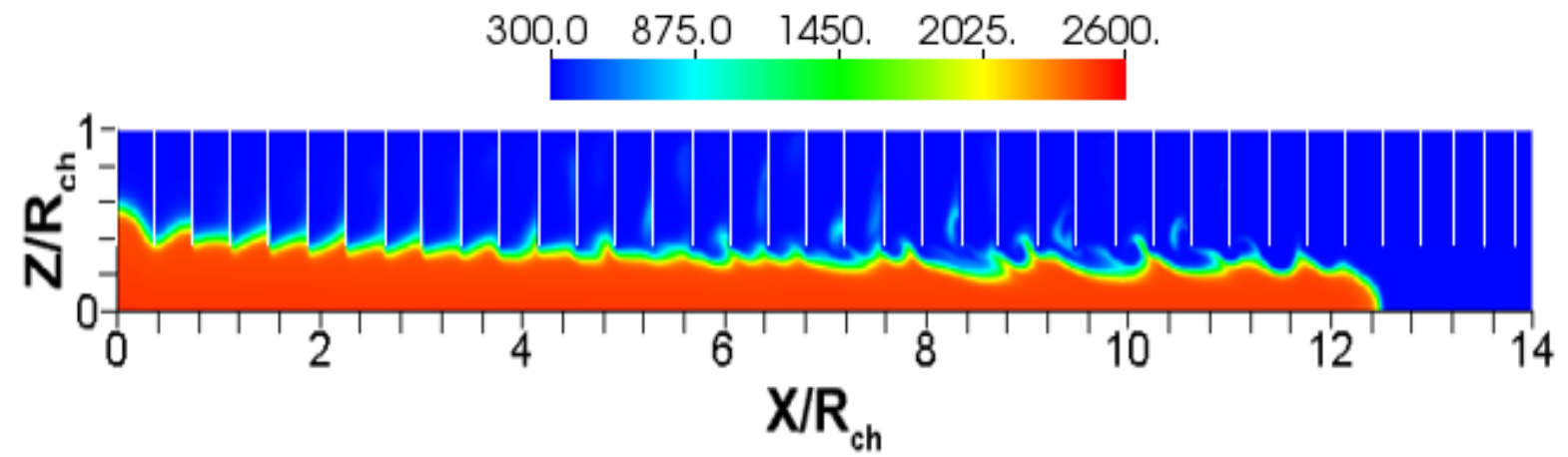

a)

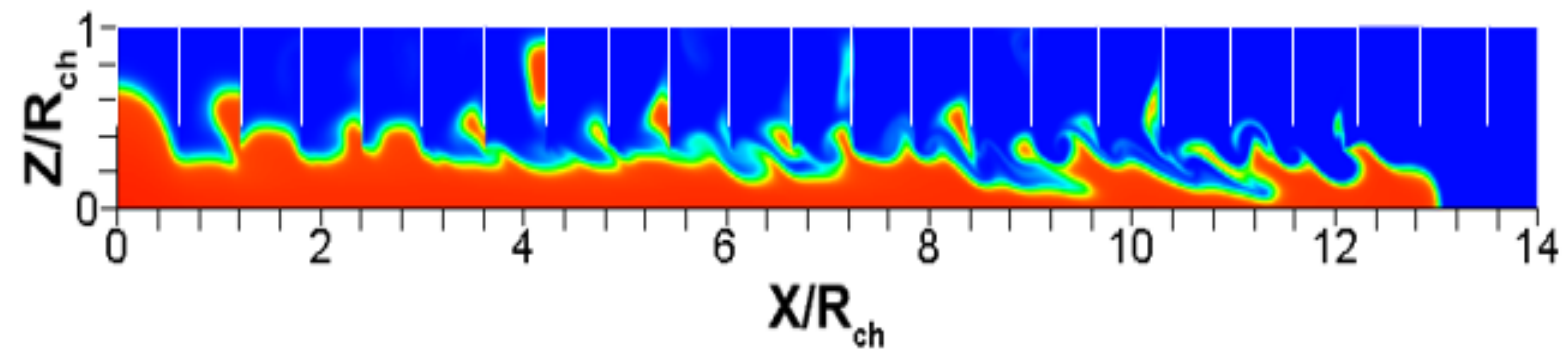

b)

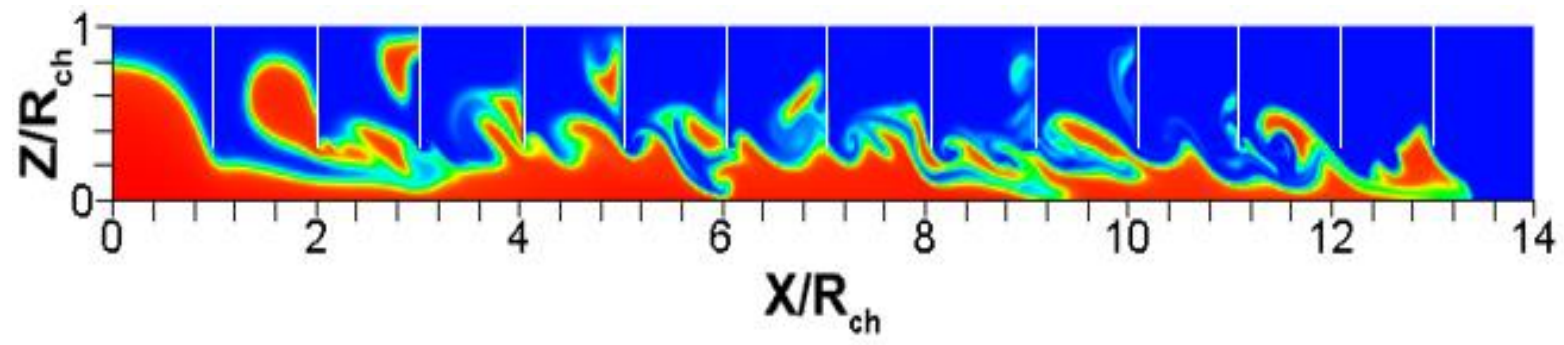

c)

Figure 4.46. Temperature distribution in obstructed channels (upper half shown), $R_{c h}=24 L_{f}$, $\alpha=2 / 3$, and different spacing: a) $\Delta Z / R_{c h}=0.38$, b) $\Delta Z / R_{c h}=0.6$, and c) $\Delta Z / R_{c h}=1$.

Having explored the geometrical variables of the obstructed channel and their effect on the flame shape and velocity, it seems interesting to extend the analysis to the effect of heat exchange at the walls. The procedure is similar to that of unobstructed channels, namely, isothermal 
conditions are considered at the walls, with the obstacles behaving adiabatically, since the thermal capacity of such thin elements is expected to be negligible.

\subsubsection{Isothermal Walls Effect}

The thermal boundary condition, added to the system by isothermal walls, does not have the importance observed in smooth-walled channels. Similarly to the case described when varying the channel width, the walls do not interact with the flame noticeably. The vertical flame propagation through the pockets occurs in a much slower pace than the horizontal one along the channel centerline, since the propagation in the pockets is produced from an open end to a wall, restricted by the burning gas as it expands in the opposite direction. Therefore, the flame reaches the wall subjected to a constant temperature at a later stage, resulting in a poor contribution of the thermal energy exchanged at the walls on the flame propagation.

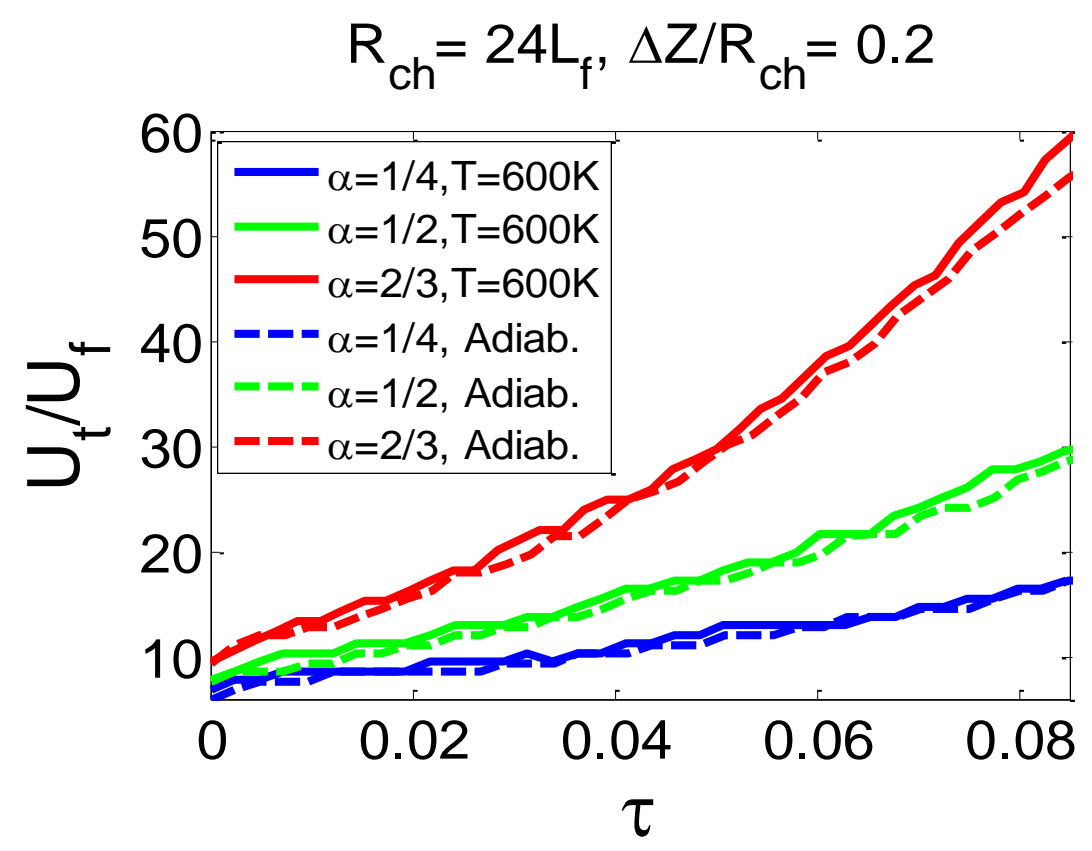

Figure 4.47. Flame tip velocities obtained in obstructed channels considering different wall thermal conditions: isothermal conditions (solid) and adiabatic conditions (dashed).

In Figure 4.47, the minor importance of the heat exchange at the walls is shown by comparing channels with adiabatic and isothermal walls, including different blockage ratios: $\alpha=1 / 4, \alpha=$ $1 / 2$ and $\alpha=2 / 3$. The trends change basically with the obstacle size, even though the wall temperature considered is high, $600 \mathrm{~K}$. The conclusion is reinforced by the results shown in 
Figure 4.48, where in addition to the adiabatic condition, the obstructed channel has been exposed to three wall temperatures, starting with the cold condition $298 \mathrm{~K}$, including $600 \mathrm{~K}$ and $1000 \mathrm{~K}$. The major difference is given by the $T_{w}=1000 \mathrm{~K}$ case, where a $15 \%$ increase in the flame propagation velocity respect to the adiabatic case is observed.

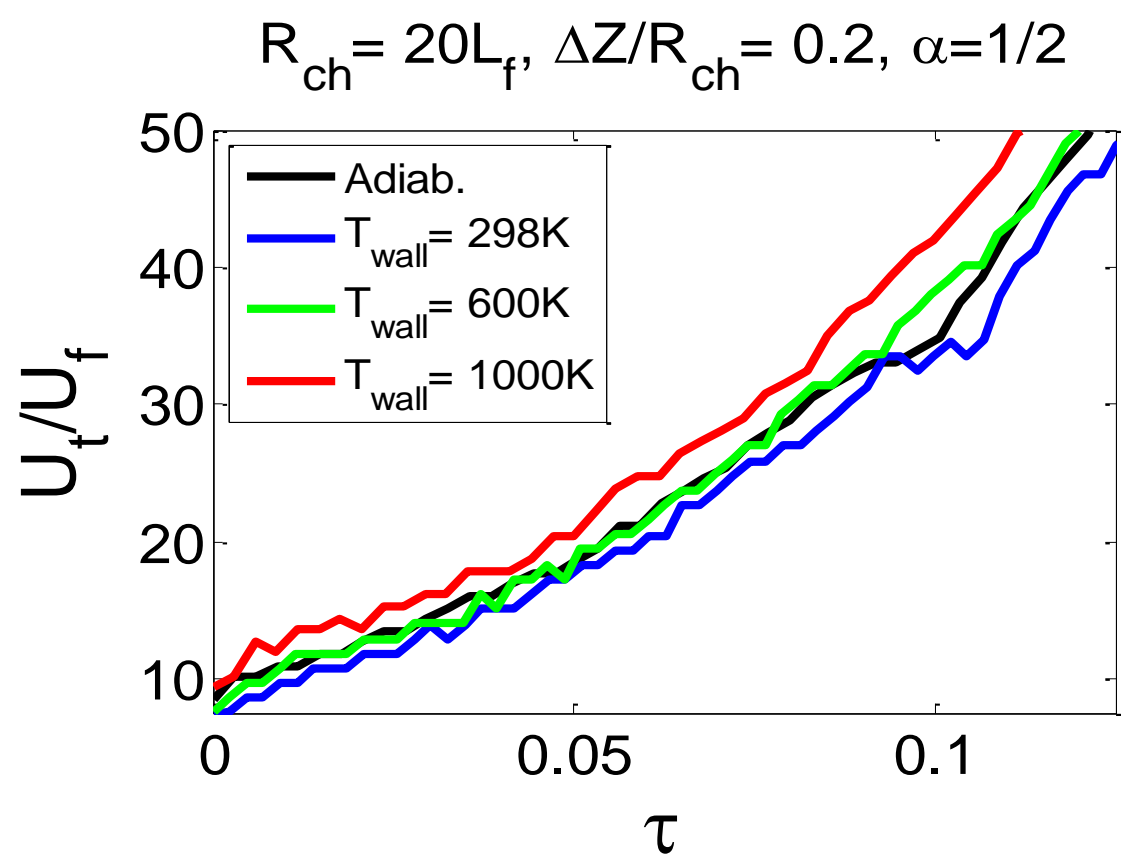

Figure 4.48. Flame tip velocities in a channel considering different wall temperatures.

Figure 4.49 shows a comparison of the gas flow produced at isothermal and adiabatic conditions. Although a gradient of temperature is established in the isothermal case within the pockets, the generated flow is conceptually the same in both cases. A flow circulation is produced as the propagating flame compresses the unburned gas against the wall, which is added to the central region, transferring momentum to the flame propagation therein. Moreover, the flame propagates along the channel in a laminar fashion, since the vortices generated in the pockets do not elongate or distort the flame surface area in the channel center.

In this respect, it can be concluded that the thermal energy transfer at the walls does not modify the flame morphology and dynamics significantly. Obviously, this includes the extreme conditions for flame extinction and stretching along the walls observed in smooth wall channels. Therefore, the geometrical boundary conditions controlling the momentum exchange at the channel walls are the key parameters in this configuration. 


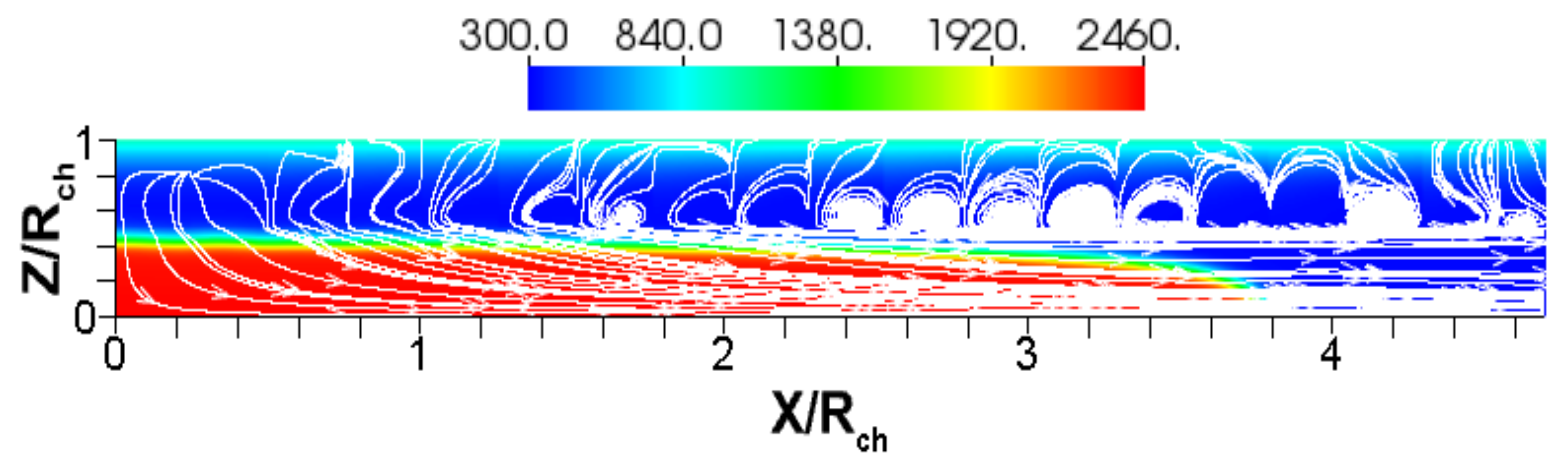

a)

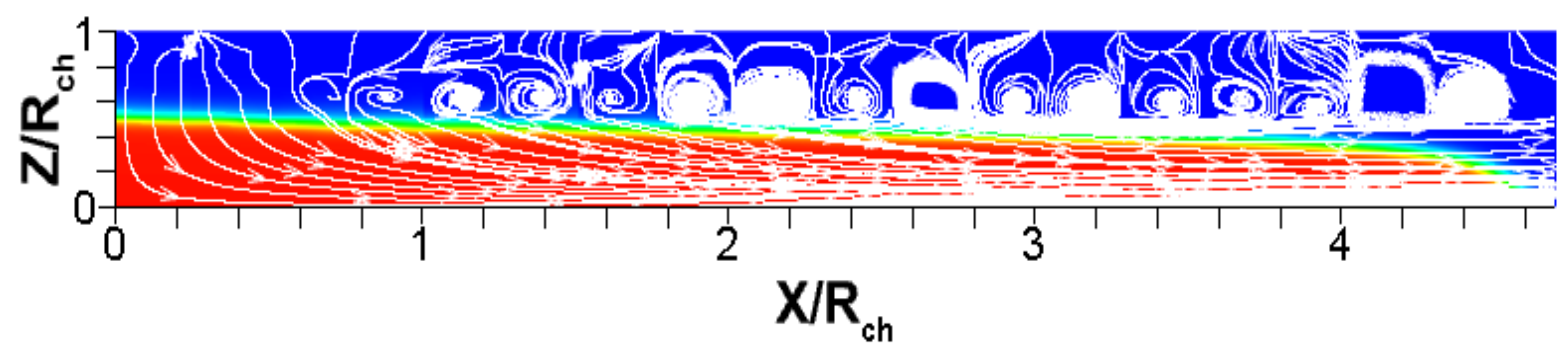

b)

Figure 4.49. Instantaneous temperature distributions and streamlines describing the flame propagation in an obstructed channel given by $R_{c h}=20 L_{f}, \alpha=1 / 2, \Delta Z / R_{c h}=0.2$. a) $T_{w}=1000 \mathrm{~K}$, b) adiabatic walls.

It is noticed that the intense flame acceleration observed in obstructed channels depends on the geometry of the pocket, as defined by the blockage ratio $\alpha$ and the distance between obstacles $\Delta \mathrm{Z}$. The flame velocity can be decreased significantly by considering smaller obstacles sizes, given a moderate separation between obstacles. However, the flame acceleration is not effectively reduced when the obstacle size considered is smaller than a certain value, e.g. $\alpha=$ 1/9 for the conditions specified in Figure 4.39. In the next section, small obstacle sizes are studied giving emphasis to the later stages, which will also help to identify the limitations of the geometrical conditions impact on the flame dynamics.

\subsubsection{Finger Flame Limit}

In Section 4.2.1, it was discussed how the blockage ratio can modify the velocity of the flame propagating in the central region of the channel. Indeed, it was shown in Figure 4.39 that once the obstacle size is reduced to a certain point (e.g. $25 \%$ of the channel width), further reduction of the blockage ratio does not provide significant influence on the flame velocity. This tendency is explored in Figure 4.50, by considering smaller blockage ratios, $\alpha=1 / 40$ and $\alpha=1 / 20$, 
with the obstacle separation being $\Delta Z / R_{c h}=0.6$. The flame velocity grows exponentially with time until $\tau \sim 0.27$, where the onset of a much weaker propagation is noticed. Then, the velocity trends are replaced by slower (linear) velocities, which slope is smaller as the blockage ratio is reduced. In the case of $2.5 \%$ blockage ratio, $\alpha=1 / 40$, this change is significantly more abrupt than that for $5 \%$ blockage ratio, $\alpha=1 / 20$, including a stage of a quasi-steady flame propagation and even a slight velocity reduction, before the initiation of the linear trend. To understand the reason for such a change in the flame propagation, it is necessary to recall the conditions at which the process is been produced, with a special attention to the manner it is initiated.

The combustion event starts with a spherical flame embryo at the channel centerline. As a result, the flame front propagates in the obstructed channel with slip surfaces. These are the same conditions considered to obtain the so-called finger flame propagation, with the difference that the latter is observed in unobstructed channels (Section 2.4). The finger flame model is characterized by a strong acceleration at the beginning, which is terminated once the flame skirt reaches the channel walls. In Figure 4.50, a similar behavior is observed, with a reduced velocity at the later stage. By considering small blockage ratios, the channel tends to approach the smooth wall scenario, even though the separation between obstacles is $1 / 3$ of the channel width.

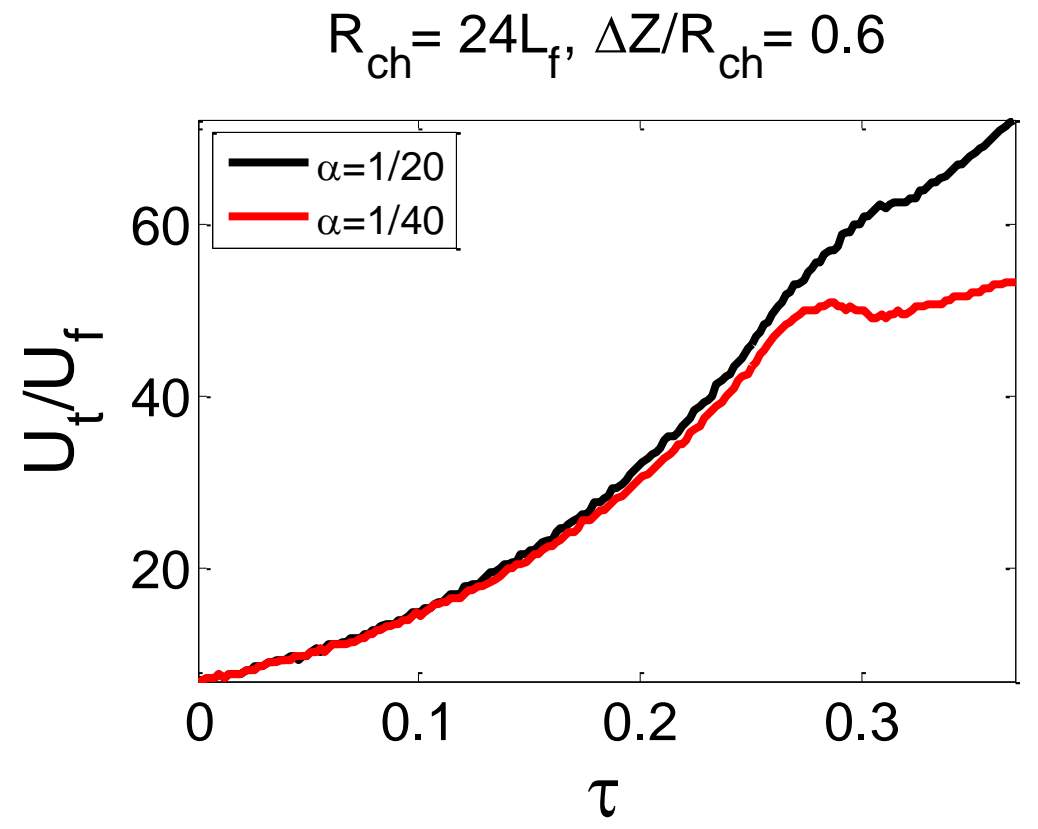

Figure 4.50. Flame tip velocities obtained in a channel considering small blockage ratios. The finger flame propagation characteristics observed in the obstructed configuration is illustrated in Figure 4.51, considering $\alpha=1 / 20$ and $\Delta Z / R_{c h}=0.6$. Initially, the flame 
propagates in two directions: along the channel centerline, and towards the wall, in a much slower manner. Since the pockets formed in these conditions are quite small, they do not contribute to enhance the flame propagation in the channel center significantly, providing slower flame propagation as compared to those shown, for example, in Figure 4.39. Moreover, there is less gas expanding in the pockets, which results in a poorer resistance to the vertical flame propagation. As a result, the flame reaches the channel walls before the flame tip can propagate much further, generating also fewer 'new' pockets ignited.

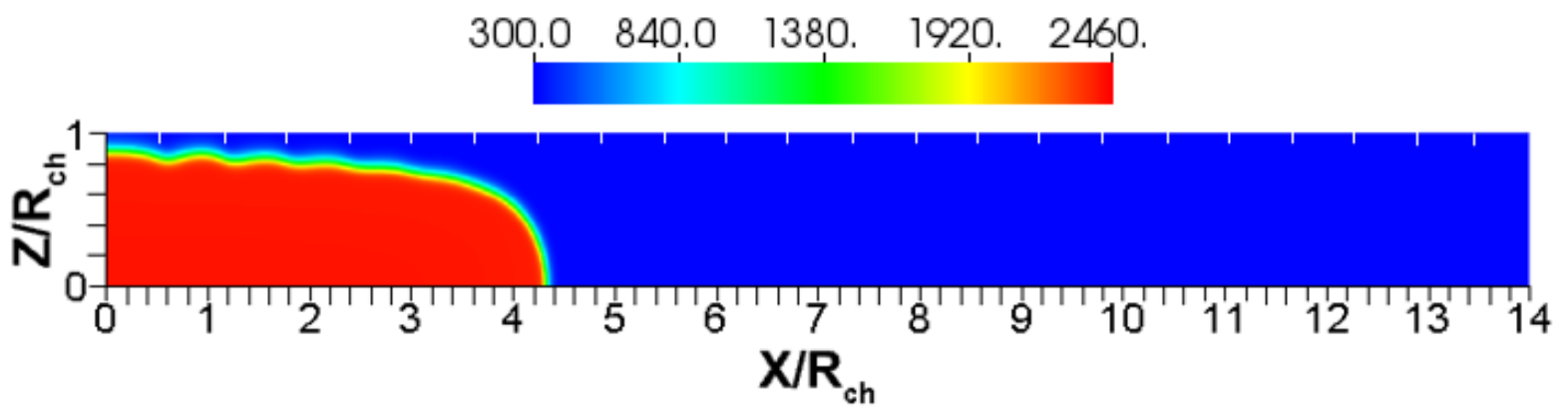

a)

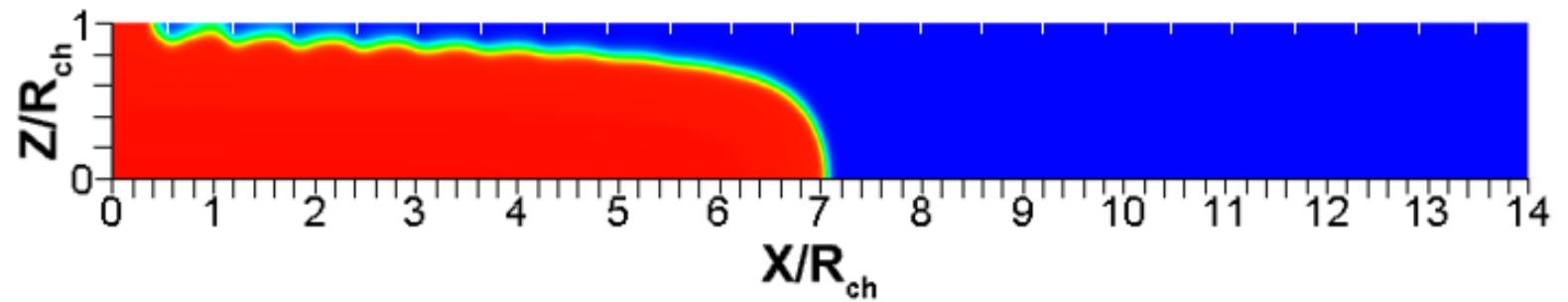

b)

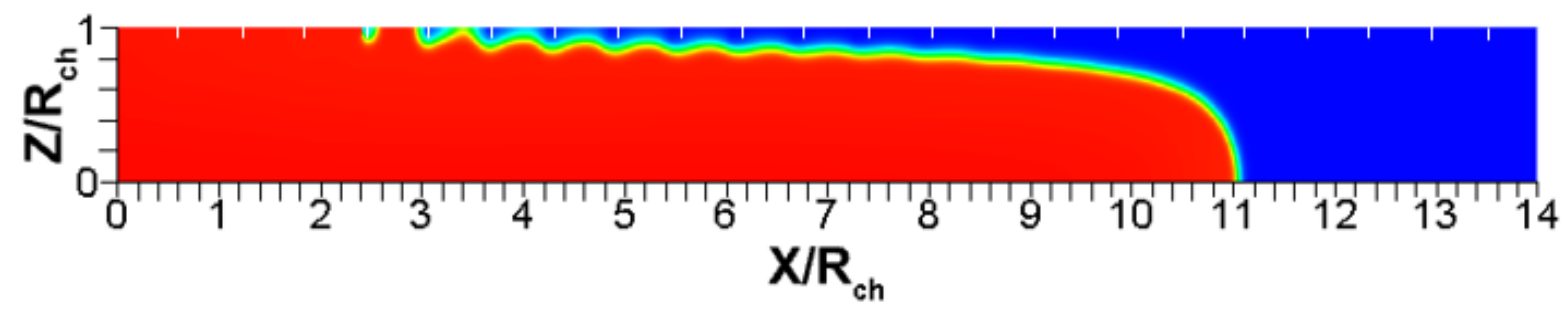

c)

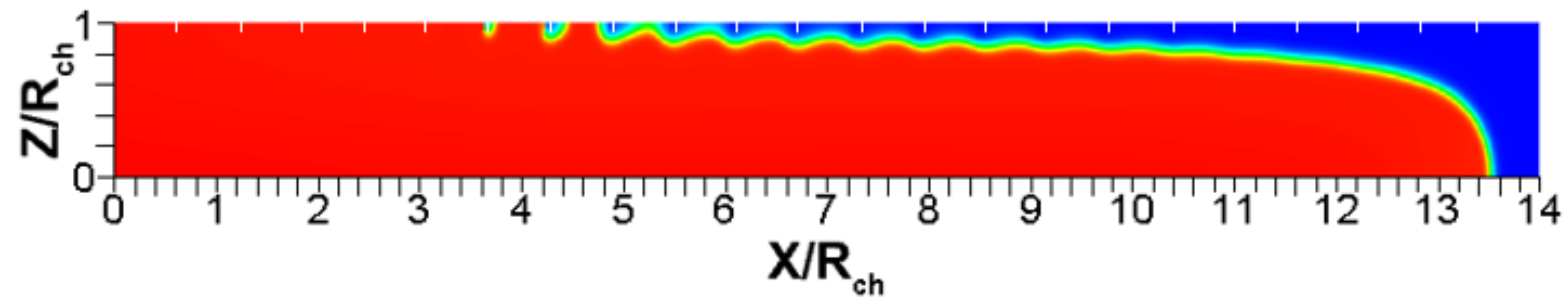

d)

Figure 4.51. Temperature distribution describing the finger flame limit in obstructed channels, $R_{c h}=24 L_{f}, \alpha=1 / 20, \Delta Z / R_{c h}=0.6$. a) $\tau=0.228$, b) $\tau=0.287$, c) $\tau=0.351$, d) $\tau=0.386$. 
But the most important consequence of having the flame reaching the channel walls is the associated flame surface reduction. The flame surface that was constantly increasing along the process (much more than, for example, when propagating in smooth channels initiated by a planar ignition), experiences this reduction once the combustion has been completed in the pockets. The flame surface reduction is produced progressively, starting with the pockets ignited earlier, and it is the responsible for the deceleration observed in the later stages of the process.

Therefore, the importance of including obstacles at the channel walls relies also on the way they delay the flame surface reduction at the walls by restricting the vertical flame propagation, allowing a sustained flame acceleration that differs from that given by the finger flame model, which is also very intense, but limited in time.

\subsubsection{Discussion}

While examining the effects of structural and thermal boundary conditions on the combustion process, some observations have been made in terms of combustion regimes, flame propagation velocities, and dimension limitations. In this section, these observations are extended to include a more detailed analysis, which include the quantification of such effects.

\subsubsection{Laminar versus Turbulent Regimes}

It was shown in Figure 4.46 how certain pocket dimensions can produce conditions for turbulent combustion. By increasing the obstacles spacing and length, the flame propagation tends to be more random, and a significant enhancing in the associated flame velocity is obtained. Certainly, the combustion process is expected to reach turbulent regimes as a result of the intense acceleration, if the process continues in this obstructed configuration. For the early stages studied here, however, some questions can be formulated regarding this early transition. For example, is it possible to determine when the transition will occur for a given pocket size? Also, what pocket dimensions promote it?

To address these questions, the numerical results have been compared to those given by an analytical formulation, which was derived considering a laminar flow approximation [42]. Specifically, the evolution equation for the flame tip position reads:

$$
\frac{d Z_{f, \text { channel }}}{d t}=\frac{(\Theta-1) U_{f}}{(1-\alpha) R} Z_{f, \text { channel }}+\Theta U_{f},
$$


with the solution as shown in Section 2.6

$$
\frac{Z_{f, \text { channel }}}{(1-\alpha) R}=\frac{\Theta}{\Theta-1}\left[\exp \left(\sigma U_{f} t / R\right)-1\right],
$$

where $\sigma_{\text {channel }}=(\Theta-1) /(1-\alpha)$, Equation 11, describes the exponential flame acceleration rate. In the numerical simulations, the flame tip position $\left(Z_{f, \text { channel }}\right)$ is determined by $R_{t}$ and the 'radius' $R$ (half-width) of the channel by $R_{c h}$. In both cases, the planar flame velocity is given by $U_{f}, \Theta$ is the thermal expansion coefficient and $\alpha$ is the blockage ratio.

The comparison is initiated in Figure 4.52, considering different blockage ratios $\alpha=$ $2 / 3,1 / 2,1 / 5$ with the obstacles spacing $\Delta Z / R_{c h}=0.38$. For such proximity between obstacles, the laminar approximation agrees with the simulations quite well, especially for moderate $\alpha$ values. Also, the generation of turbulent combustion may be inferred when the obstacle length is increased $(\alpha=2 / 3)$, where a deteriorated agreement is noticed. These results demonstrate the faster transition from laminar to turbulent regimes produced as the blockage ratio is increased, which complement the flame behavior observed in Figure 4.46.

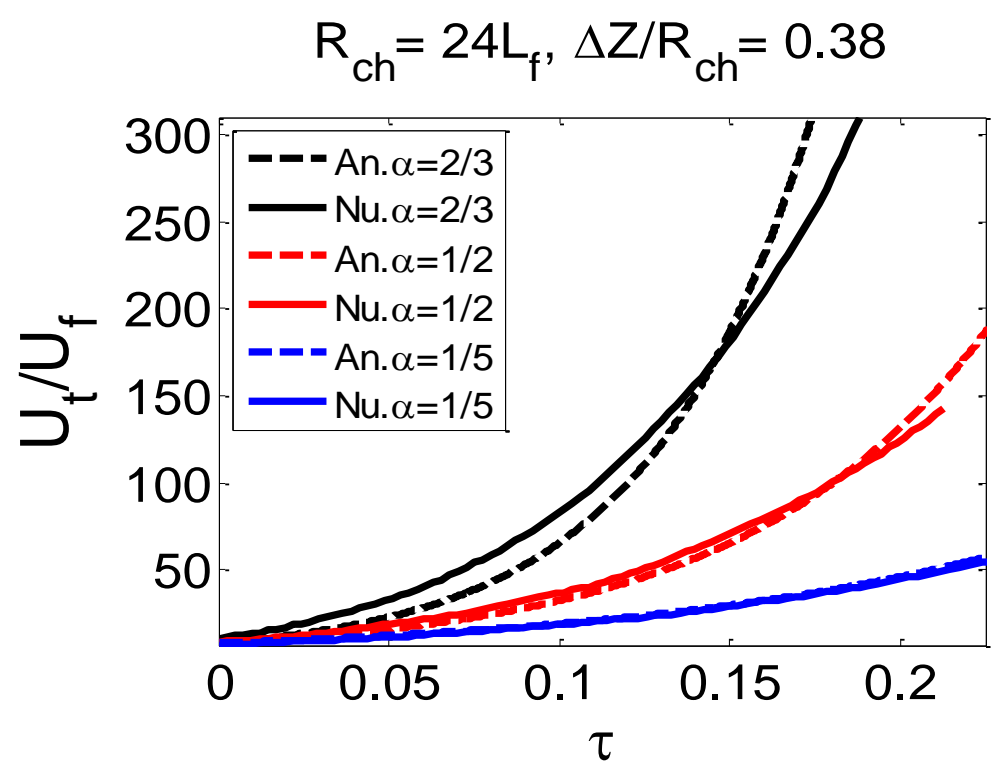

Figure 4.52. Flame velocities calculated numerically and analytically (Eq. 24), $\Delta Z / R_{c h}=0.38$. In Figure 4.53, the same blockage ratios are examined for a larger separation, $\Delta Z / R_{c h}=0.6$. By raising the width of the pockets, turbulence is enhanced, and the agreement between analytical and numerical results deteriorates even for a smaller blockage ratio, $\alpha=1 / 2$. Moreover, the numerical simulations provide larger velocities than that predicted by the laminar theory when turbulence is generated, which agrees with a more intense combustion process associated with 
turbulent regimes. Also, it is noticed that even with this larger separation between obstacles in place, a quasi-laminar propagation is still possible, as shown by the blue line, $\alpha=1 / 5$.

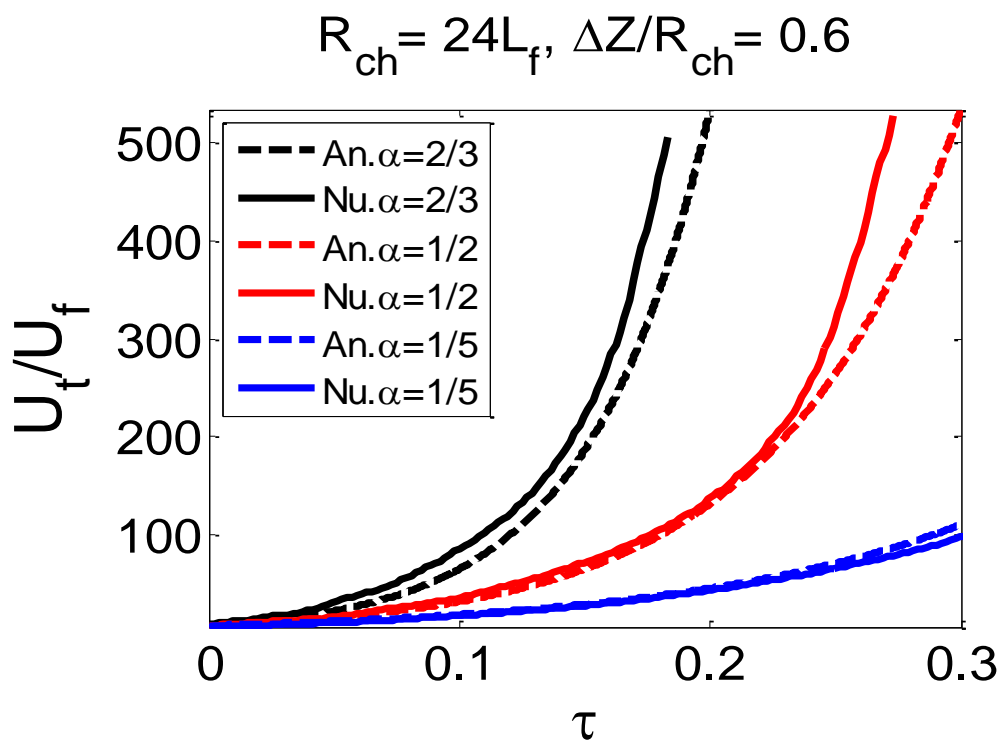

Figure 4.53. Flame velocities calculated numerically and analytically (Eq. 24), $\Delta Z / R_{c h}=0.6$ In principle, the transition from laminar to turbulent combustion regimes was associated with the lack of similarity between numerical and theoretical results. However, this can be corroborated by the associated temperature snapshots and streamlines, as seen in Figure 4.54:

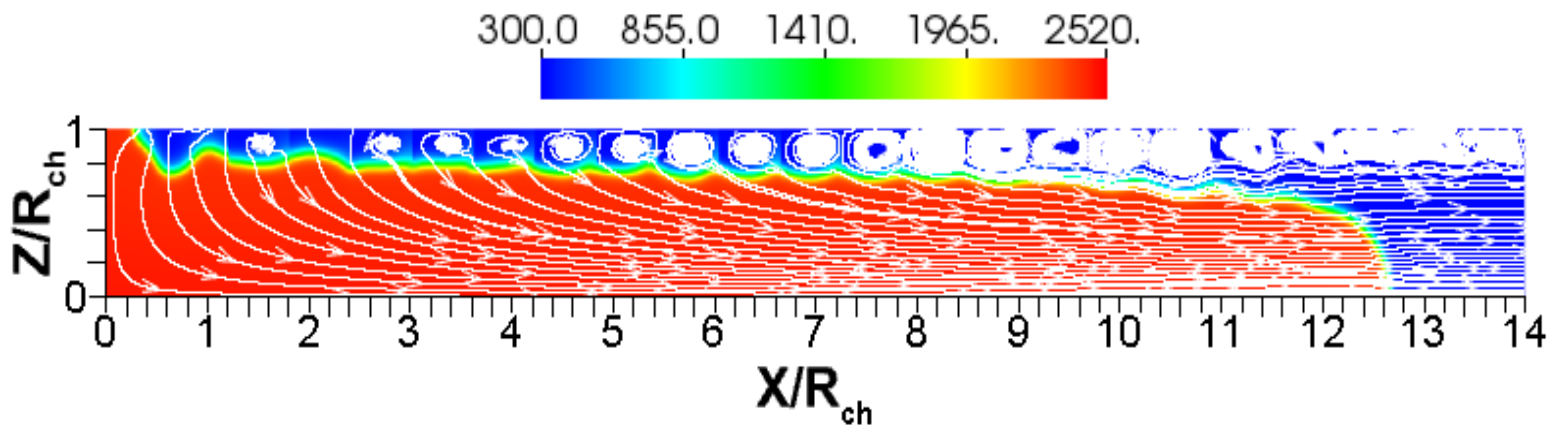

a)

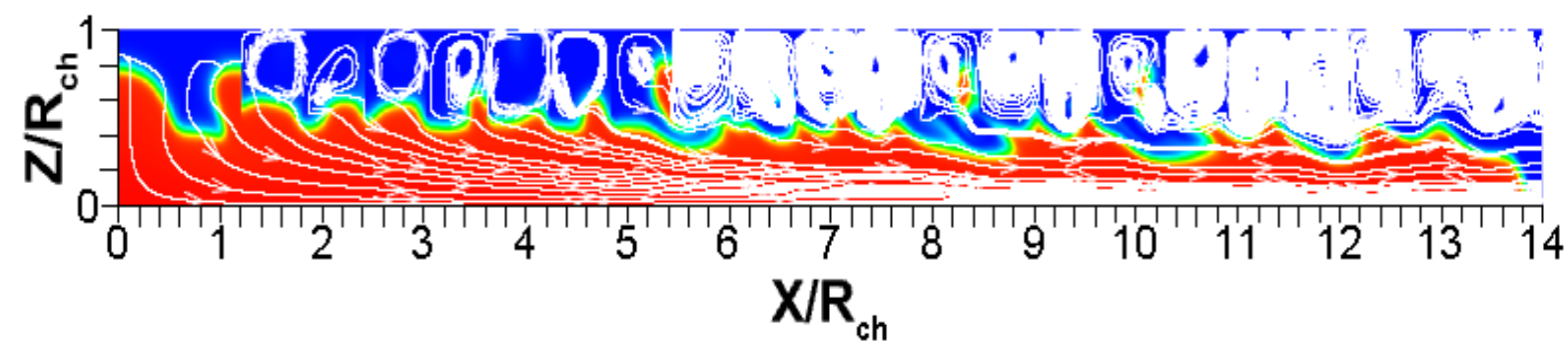

b)

Figure 4.54. Temperature distribution and streamlines describing laminar and turbulent flame propagation regimes. $R_{c h}=24 L_{f}, \Delta Z / R_{c h}=0.6$, a) $\alpha=1 / 5$, b) $\alpha=1 / 2$. 
Figure 4.54 compares the cases associated with the red and blue solid lines in Figure 4.53. It is noticed how the generated gas flow changes when increasing the obstacle length. To be specific, small obstacles promote vortices within the pockets, however, the confinement restricts the vortices length, and thereby the momentum transferred to the flame surface, expressed in the moderate distortion that the flame shape exhibits (Figure 4.54a). On the contrary, larger obstacles generate the necessary space for the development of bigger eddies in the pockets, which corrugates and distorts the flame surface more intensely, as seen in Figure 4.54b.

The turbulence generation is enhanced when the separation between obstacles is further extended, as seen in Figure 4.55. It shows the flame velocities generated in channels with the blockage ratios considered in Figures 4.52 and 4.53, but with a larger separation, $\Delta Z / R_{c h}=1$. In this case, the numerical results deviate from the laminar prediction earlier for the blockage ratios $\alpha=2 / 3$ and $\alpha=1 / 2$. However, the transition from laminar to turbulent still is not produced when the obstacle length is kept small ( $\alpha=1 / 5$, blue line).

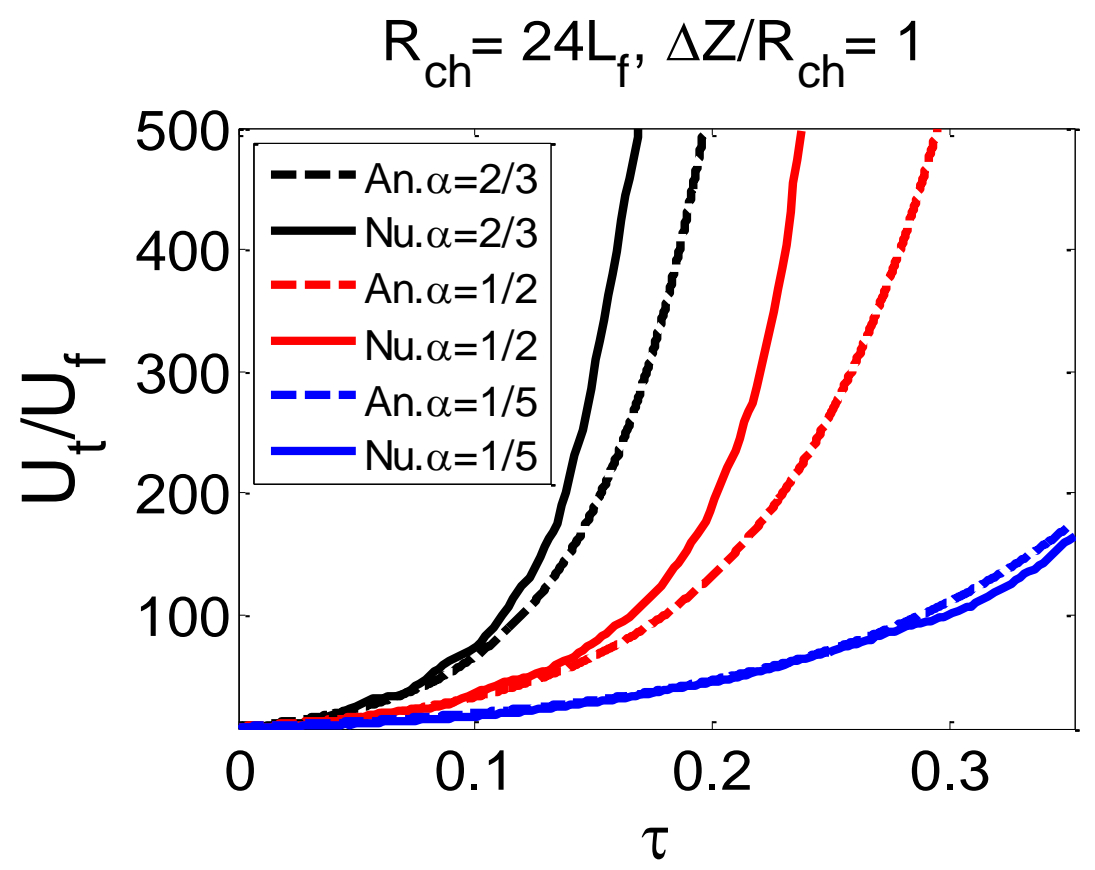

Figure 4.55. Flame tip velocities found numerically and analytically (Eq. 24) for various $\alpha$.

Figure 4.56 shows the temperature distribution and the streamlines describing the cases represented by the blue and red lines in Figure 4.55. It is seen that the flame becomes more corrugated towards the walls in both cases as compared to Figure 4.54, and the length of the 
generated eddies proportionally enlarged. At this point, it seems that the flame corrugation and the possibility of a turbulent combustion regime depend on the blockage ratio rather than on the distance between the obstacles. The vertical length of the generated eddies determines the momentum transferred to the flame surface that can effectively distort it. In the horizontal direction, this momentum exchange basically produces a shear force on the flame surface, which does not corrugate it significantly.

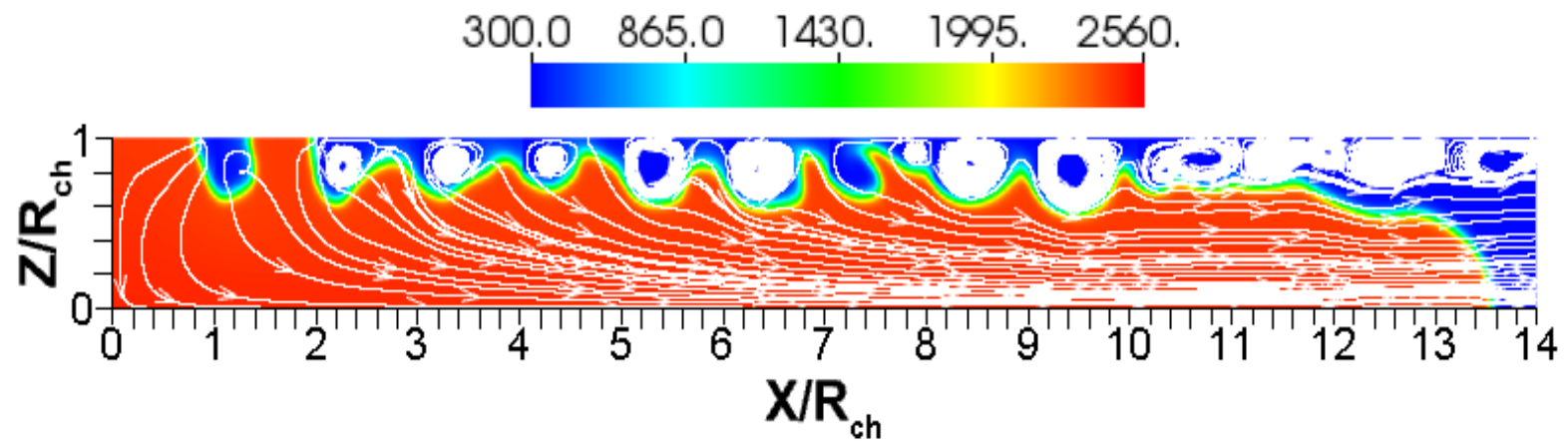

a)

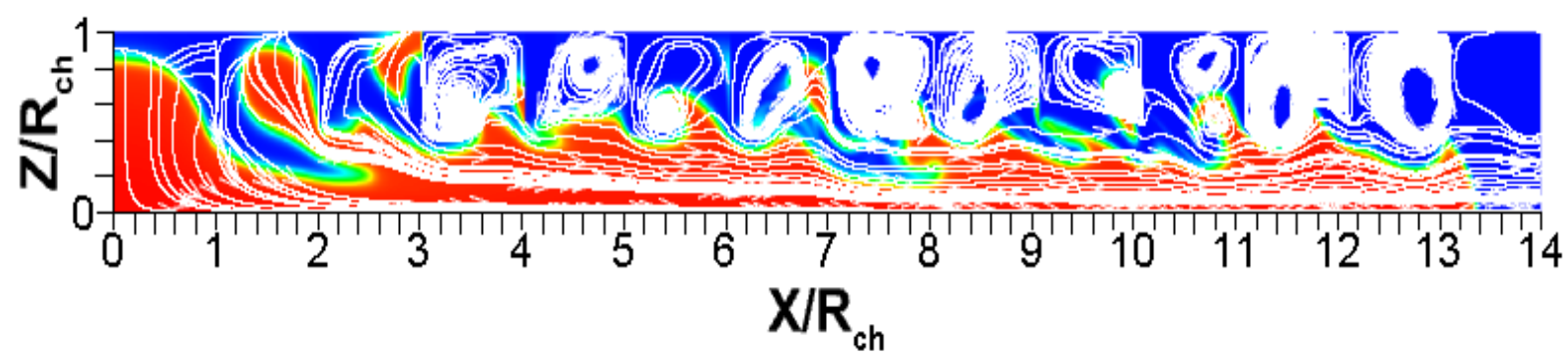

b)

Figure 4.56. Temperature distributions and streamlines describing the laminar and turbulent regimes obtained in obstructed channels. $R_{c h}=24 L_{f}, \Delta Z / R_{c h}=1$, a) $\alpha=1 / 5$, b) $\alpha=1 / 2$.

The fact that the laminar regime is preserved when small obstacles are employed (e.g. $\alpha=1 / 5$ ), even for the larger obstacles separations, provides an important parameter value to establish the limit between the laminar and turbulent regimes. In the next section, the numerical results are used to identify three regimes of flame propagation in obstructed channels, where the finger flame limit, observed for very small blockage ratios, is also included.

\subsubsection{Flame Propagation Regimes in Obstructed Channels}

The results of the present parametric study have been used to identify three domains, describing the regimes at which the flame propagates in obstructed channels in early stages: (i) turbulent 
regime, (ii) laminar regime, and a third one establishing the limit for the sustained acceleration of this mechanism: (iii) finger flame limit. These regions, in turn, are based on the parameters defining the pockets geometry; namely, the obstacles separation $\Delta Z$, and the obstacle length, given by the blockage ratio $\alpha$.

In a recent work [65], the flame acceleration obtained in obstructed pipes with very small blockage ratios has been found to be proportional to the acceleration rate $\sigma$, given by

$$
\sigma \approx \frac{2}{\alpha}\left[1-\frac{1}{\alpha(\Theta-1)}\right],
$$

from which it is possible to obtain the critical obstacle size at which the strong flame acceleration in the obstructed scenario can be supported, before turning to the finger flame model. This limit is seen at $\sigma=0$, where the corresponding critical blockage ratio is found as

$$
\alpha_{c}=\frac{1}{\Theta-1} .
$$

The result given by Equation 26 has been included in the proposed diagram (Figure 4.57), based on the thermal expansion coefficient considered in these simulations, $\Theta=8$.

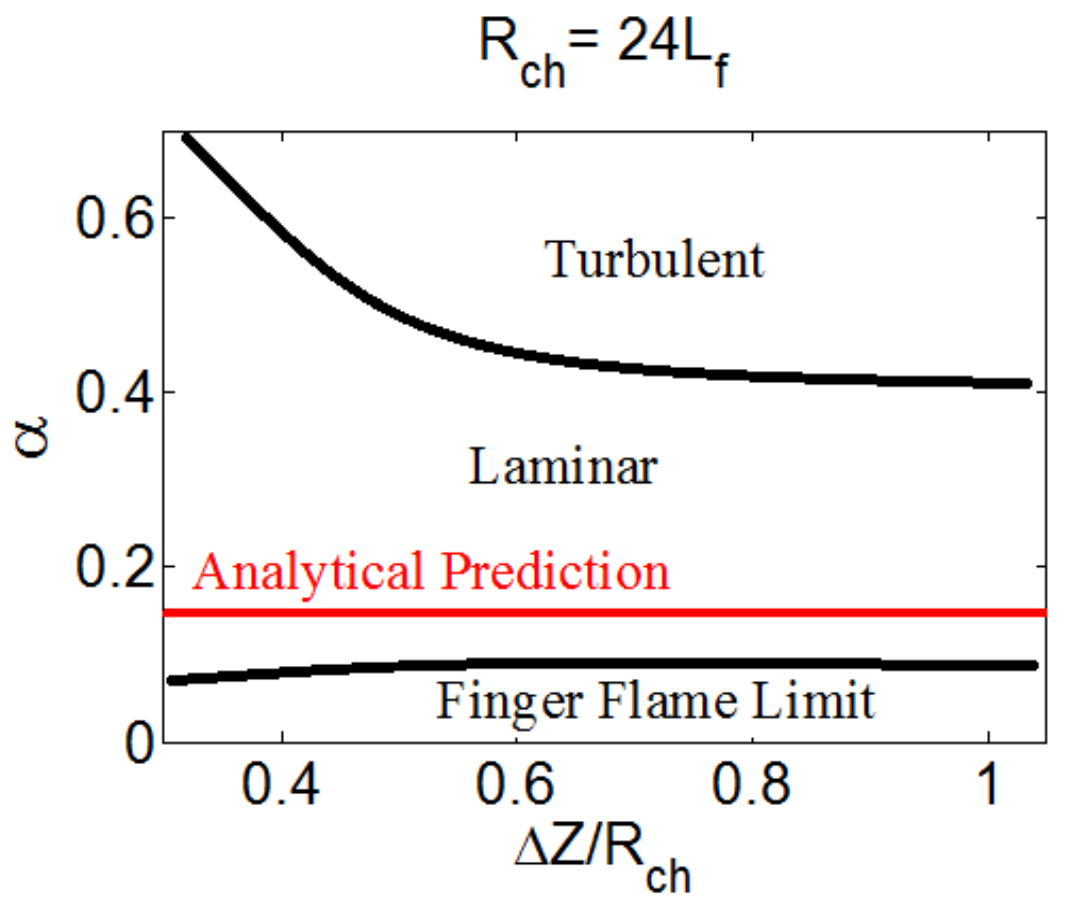

Figure 4.57. Flame regimes in obstructed channels. Analytical prediction given by Eq. 26. The diagram above shows the generation of turbulent flames for large blockage ratios, and for smaller ones in case the separation between obstacles is increased. Laminar regimes are observed 
for smaller blockage ratios, within the range of obstacles separation considered in this study. In addition, the critical blockage ratio $\alpha_{c}$ (Equation 26), yielding the limit at an exponential flame acceleration is produced, is close to the finger flame limit observed in the simulations.

To conclude, obstructed channel conditions have been observed to determine strongly accelerated flame propagation. In this sense, a better indication of this 'strength' can be found by comparing the attained flame velocities to that produced as a result of the wall friction, investigated in Section 4.1 for adiabatic and isothermal conditions. In this case, the comparison between these two mechanisms of flame acceleration is performed considering adiabatic walls.

\subsubsection{Flame Acceleration Comparison: Obstacles vs Wall Friction}

The flame velocities produced in obstructed and in nonslip wall conditions have an exponential character, as shown in Figure 4.58 and 4.59. Since the channel width is relevant for the frictional mechanism, it was necessary to consider smooth channels where the widths were the height of the free part of the obstructed channels. The smooth channel width was then calculated as $\mathrm{W}_{c h}=$ $2 \mathrm{R}_{c h}(1-\alpha)$, where $\mathrm{R}_{c h}=24 \mathrm{~L}_{f}$ is half the width of the obstructed channels. Therefore, the obstructed scenarios given by $\alpha=2 / 3$ (black line), $\alpha=1 / 2$ (red line) and $\alpha=1 / 40$ (blue line) were compared to smooth channels $16 L_{f}, 24 L_{f}$ and $46.8 L_{f}$ width, respectively.

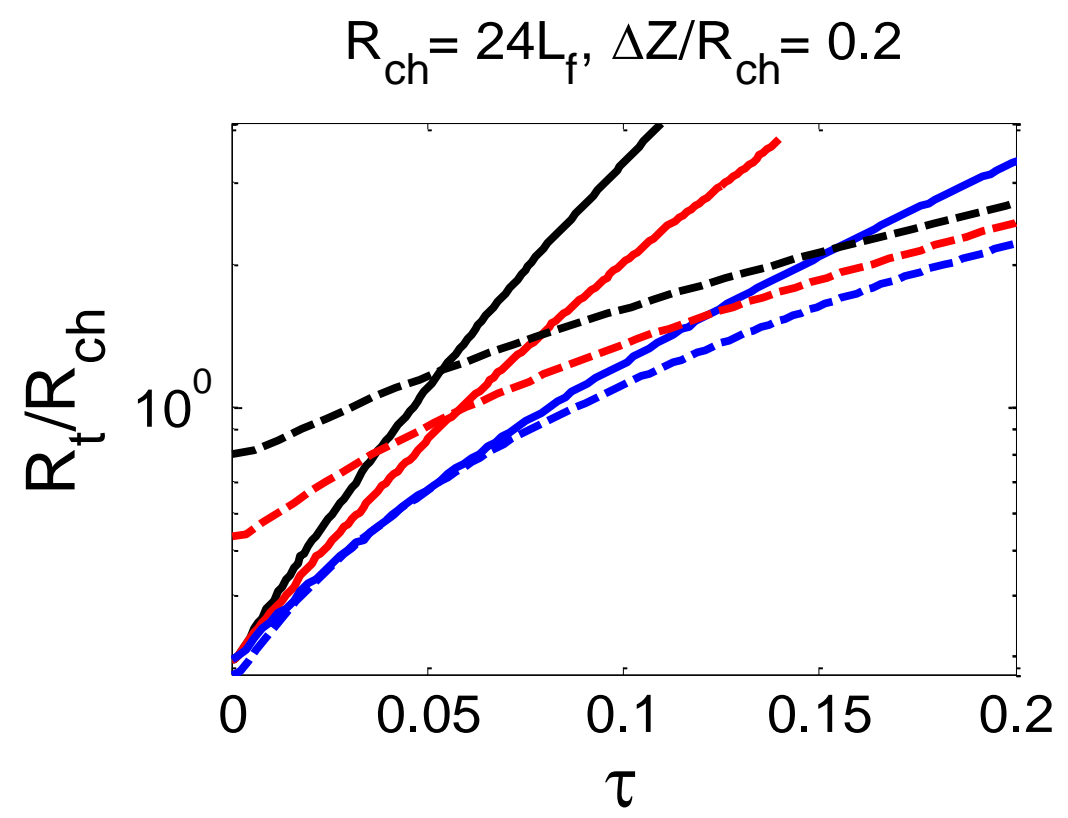

Figure 4.58. Flame tip position obtained in obstructed (solid) and in 'smooth' channels (dashed). Black: $\alpha=2 / 3, \mathrm{~W}_{c h}=16 \mathrm{~L}_{f}$; red: $\alpha=1 / 2, \mathrm{~W}_{c h}=24 \mathrm{~L}_{f}$ and blue: $\alpha=1 / 40, \mathrm{~W}_{c h}=46.8 \mathrm{~L}_{f}$. 


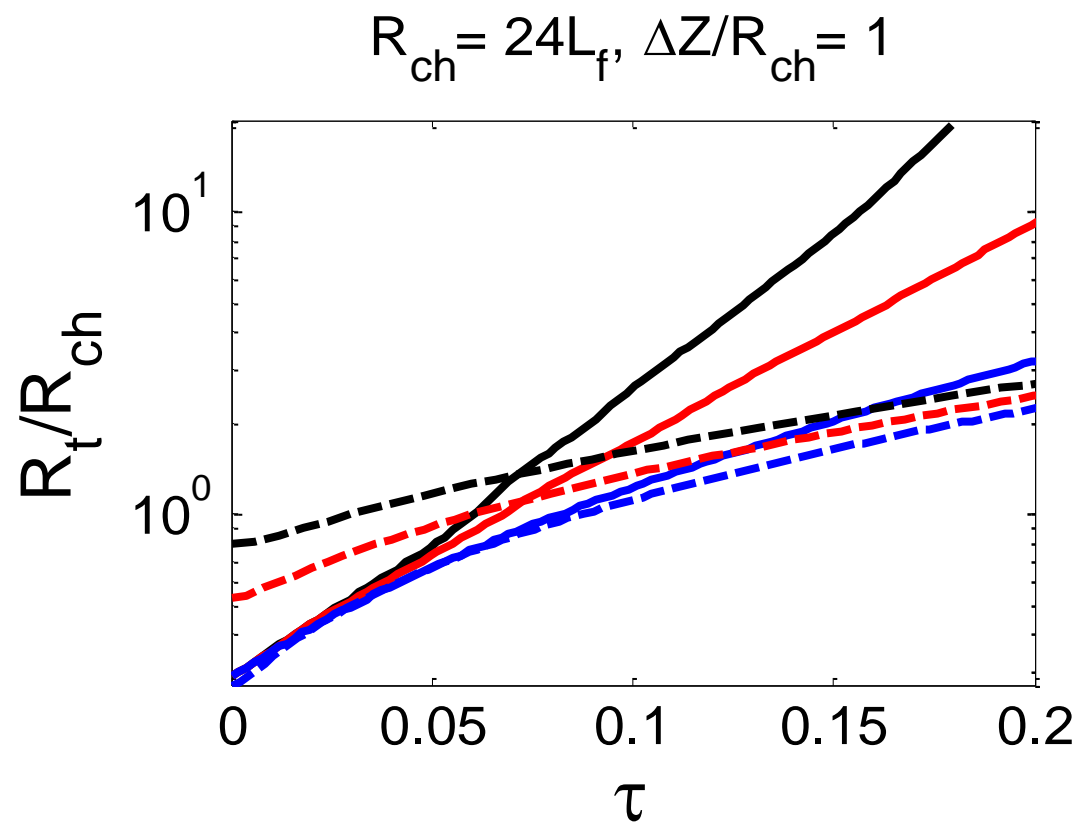

Figure 4.59. Flame tip position obtained in obstructed (solid) and in 'smooth' channels (dashed). Black: $\alpha=2 / 3, \mathrm{~W}_{c h}=16 \mathrm{~L}_{f} ;$ red: $\alpha=1 / 2, \mathrm{~W}_{c h}=24 \mathrm{~L}_{f}$ and blue: $\alpha=1 / 40, \mathrm{~W}_{c h}=46.8 \mathrm{~L}_{f}$. The comparison shows much faster flame propagation in obstructed channels, at the two obstacles spacing considered, $\Delta Z / R_{c h}=0.2$ (Figure 4.58) and $\Delta Z / R_{c h}=1$ (Figure 4.59). At a given time, the flame tip position, $R_{t}$, can be calculated in relation to the thermal expansion $\Theta$, the planar flame velocity $U_{f}$ and the total propagation flame velocity respect to the fuel $U_{w}$, as:

$$
\frac{d R_{t}}{d \tau}=\Theta \frac{U_{w}}{U_{f}},
$$

where $\tau=t U_{f} / R_{c h}$. By considering the exponential trend produced by the two mechanisms, the flame tip position can be expressed as:

$$
R_{t}=\frac{\Theta}{\sigma}[\exp (\sigma \tau)-1],
$$

Therefore, the strength of the acceleration is given by the value of the acceleration rate $\sigma$. The acceleration strength parameter $\sigma$ is presented in Figure 4.60, versus the blockage ratio at fixed obstacle separations and the corresponding smooth channel widths. It is seen that the flame acceleration rate is an order of magnitude larger for obstructed channels as compared to smooth 
channels, proving the ultra-fast flame propagation that can develop by including this structural change on the system boundaries.

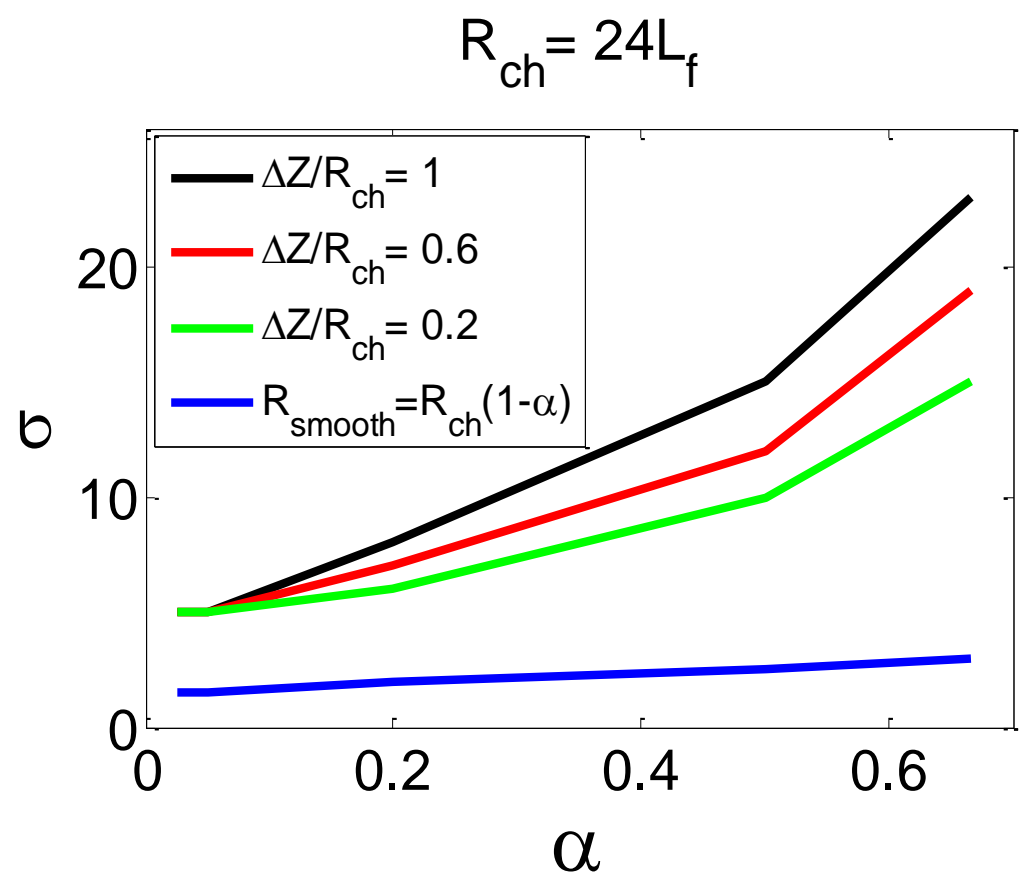

Figure 4.60. Comparison of the flame acceleration produced by obstructed and frictional walls. 


\section{Conclusions and Future Work}

\subsection{Conclusions: 'Smooth' Pipes}

The effects of isothermal boundary conditions on the flame propagation dynamics have been investigated numerically. The variables explored in the parametric study included adiabatic and isothermal wall conditions, the fuel thermal expansion and the channel width. The isothermal condition was employed at different wall temperatures, varying from the 'cold' wall condition $300 \mathrm{~K}$ (i.e. the room temperature) to the high wall temperature $1200 \mathrm{~K}$. The considered channel widths varied from $10 L_{f}$ to $60 L_{f}$, where $L_{f}$ is the flame thickness. In turn, the fuel mixture was characterized by the thermal expansion coefficient values $\Theta=5-10$, with $\Theta=\rho_{f} / \rho_{b}$.

The flame velocities obtained in isothermal pipes were much slower than that obtained in adiabatic pipes. While adiabatic walls provide conditions for an exponential increase in the flame velocity with time, the isothermal walls allow at most a linear acceleration, when colder wall conditions are in place.

It was nevertheless noticed that isothermal conditions can produce a corrugation on the flame front independently of the non-slip condition at the walls. This corrugation is the result of forcing the walls to be at a certain temperature along the process, quenching the flame front and reducing the burned gas temperature next to it.

Larger flame corrugations are observed to occur when colder walls are in place. This elongation is initially comparable with that produced at adiabatic conditions, but unlike the adiabatic case, the flame does not continue stretching with time, but provides a flame front shape that remains steady afterwards.

The flame corrugation is reduced as the wall temperature increases; the corrugation occurs until a high enough temperature is reached, where the flame starts stretching along the wall. In the latter case, the associated flame velocity becomes very large, but with an unstable behavior, including a dramatic reduction once the flame vertical expansion fills the channel section completely. 
The linear flame acceleration rate, when produced in channels of width $20 L_{f}$ for the fuel mixture characterized by $\Theta=7$, depends on the wall temperature in a near quadratic fashion. The acceleration slope can be reduced by $50 \%$ when raising the wall temperature from $300 \mathrm{~K}$ to $400 \mathrm{~K}$, with an almost constant value when the walls are kept at $600 \mathrm{~K}$.

The thermal expansion coefficient $\Theta$ has demonstrated the capacity of modifying the flame propagation velocity by setting the thermal energy limit available in the system, released by the flame front as the combustion reaction takes place.

The channel width can also modify the propagation velocity by determining how far the isothermal walls are from the inner region of the burned matter. This length determines how large the flame corrugation is as compared to the pipe section, and how efficient the post-cooling of the burned gas is, which modifies the volumetric expansion of the burned gas, an important force promoting the flame propagation.

Four distinctive flame propagation regimes have been identified in isothermal wall conditions, for flame propagation distances around $100 L_{f}-150 L_{f}$ : (i) no flame propagation or flame extinction; (ii) linear acceleration of the flame front; (iii) weak flame acceleration or almost steady flame propagation; and (iv) oscillating behavior of the flame propagation velocity.

At later stages, the combustion event experienced an extinction process in isothermal pipes. The cooling produced by the isothermal surfaces at $300 \mathrm{~K}$ led to a contraction of the burned gas volume, which produced the backwards displacement of the flame front towards the closed end.

\subsection{Conclusions: Obstructed Configuration}

The addition of obstacles to the walls of the channel has also been investigated. The study has been performed in relation to the effect that the geometric parameters as well as the thermal boundary conditions produce on the flame dynamics.

The length of the obstacles, characterized by the blockage ratio $\alpha$, can modify the flame propagation velocity significantly. The relation between this parameter and the attained flame acceleration is directly proportional, namely, the acceleration rate $\sigma$ increases linearly with the obstacle size with a value $\Delta \sigma \sim 1-2.5$ per $0.1 R_{c h}$ increase of the obstacle length, for blockage ratios $\alpha<0.5$, and $\Delta \sigma \sim 3-5$ for $\alpha>0.5$. 
The obstacles-based flame acceleration mechanism has demonstrated to be independent of the channel width, for the conditions investigated.

The flame dynamics also depends on the separation between obstacles $(\Delta \mathrm{Z})$ significantly. It has been observed that large enough separations provide conditions for turbulent regimes (i.e. $\Delta \mathrm{Z} / R_{c h}>0.6$ for $\left.\alpha=2 / 3\right)$, leading to faster flame propagation ( $40 \%$ velocity increase in a $\tau=0.05$ period after the transition from a laminar regime, for $\left.\Delta \mathrm{Z} / R_{c h}>0.6, \alpha=1 / 5\right)$.

The thermal boundary condition given by isothermal walls does not change the flame dynamics significantly $(\sim 15 \%$ peak velocity variation). The flame propagation along the axis is mainly produced far from the walls, and the heat gained by the fuel mixture in the pockets does not show an important effect on the fluid dynamics of the flow generated therein.

As the obstacles become smaller, the flame evolution approaches the finger flame model. This has been determined to occur when a blockage ratio smaller than $5 \%$ of the channel width is considered.

The transition from laminar to turbulent flame propagation regimes depends on the geometry of the pockets, formed by the obstacles and the channel walls. This transition is more easily attained when longer obstacles are in place. Also, the transition is possible when shorter ones are considered, but bigger obstacles spacing are necessary $\left(\Delta \mathrm{Z} / R_{c h}>0.6\right)$. The transition can be delayed and even not produced at these stages if small enough obstacles are included, since the pockets do not provide the necessary space for the formation of vertically long eddies that can distort the flame surface.

The flame propagation in the obstructed channel configuration is much faster than in nonslip 'smooth' channel one. The associated acceleration provided by the obstacles mechanism is one order of magnitude bigger than the frictional one, for comparable conditions. 


\subsection{Future Work}

This study, in the opinion of the author, can be extended in the following directions:

Applying the isothermal boundary conditions to the finger flame model. The author thinks it will produce a significant change on the flame dynamics in this scenario, as a result of the quenching produced at the walls, which in the absent of nonslip conditions, would produce a more sustained flame propagation.

Incorporating a variable heat transfer flux to the channel walls. This process would provide the intermediate cases between the adiabatic and isothermal conditions, given scenarios that can be more easily achieved in experiments, and offering a desired 'flexibility' to study the heat transfer interaction with other flame parameters.

Modifying the obstacle configuration in such a way that new obstacle geometries can be mounted on the system. The analysis can be greatly extended by modifying the obstacles shape, size and distribution. Some of them are currently observed in technical applications, including the mounting of the obstacles on the top or on the bottom boundary only.

Extending the parametric study to axisymmetric obstructed tube configurations. Although no major differences are expected, as seen in the 'smooth' configuration, the larger flame front surface area obtained in the cylindrical case should be studied in relation to the parameters discussed in this study.

Including variable planar flame velocities at the two conduit configurations. These can represent a number of situations where the planar flame velocity respect to the fuel mixture is not constant, e.g. a system including dust particles, inert or reacting. 


\section{References}

1. U.S. Energy Information Administration, July 2013. International Energy Outlook 2013. DOE/EIA-0484.

2. Oran, E. \& Gamezo, V., 2007. Origins of the Deflagration-to-Detonation Transition in GasPhase Combustion. Combustion and Flame, 148, pp. 4-47.

3. Zeldovich, Y. \& Frank-Kamenetski, D., 1938. A Theory of Thermal Propagation of Flame. ACTA Physico-Chimica URSS, 9(2), pp. 341-350.

4. Bychkov, V. \& Liberman, M., 2000. Dynamics and Stability of Premixed Flames. Physics Reports, 325, pp. 115-237.

5. Ro, K., Oh, J. \& Dong, L., 2007. Lessons Learned: Application of Small UAV for Urban Highway Traffic Monitoring. 45th AIAA Aerospace Sciences Meeting and Exhibit, Reno, NV.

6. Johnson, L.F., Herwitz, S., Dunagan, S., Lobitz, B., Sullivan, D. \& Slye, R., 2003. Collection of Ultra High Spatial and Spectral Resolution Image Data Over California Vineyards with a Small UAV. Proceedings of the 30th International Symposium on Remote Sensing of Environment, Honolulu, HI, November 10-14.

7. Casbeer, D.W., Beard, R.W., McLain, T.W., Li, S. \& Mehra, R.K., 2005. Forest Fire Monitoring with Multiple Small UAVs. American Control Conference, Portland, OR, June $8-10$.

8. Yu, Y., Cadou, C. \& Maruta, K., 2015. Micro Combustion and Power Generation. Momentum Press, LLC, New York.

9. Lewis, N., 2002. Portable Energy for the Dismounted Soldier. MITRE Corporation.

10. Mills, A.F., 1995. Basic Heat and Mass Transfer. Table A.25, Chicago, IL: Richard D. Irwin Inc., p. 879. 
11. Broussely, M. \& Archdale, G., 2004. Li-ion Batteries and Portable Power Source Prospects for the Next 5-10 years. Journal of Power Sources, 136, pp. 386-394.

12. Blaxter, K., 1989. Energy Metabolism in Animals and Man, Cambridge, Table A1.1, 297.

13. Edwards, T. \& Maurice, L., 2001. Surrogate Mixtures to Represent Complex Aviation and Rocket Fuels. AIAA Journal of Propulsion and Power, 17, no. 2, pp. 461-466.

14. International Energy Agency, 2010. Oil Information Documentation for Beyond 2020 Files. [Online] http://wds.iea.org/wds/pdf/documentation_oil_2010.pdf

15. Knapp, L. The Little Engine That Could Be. Wired.com, 26 November 2001. [Online]. http://archive.wired.com/science/discoveries/news/2001/11/48400?currentPage=all.

16. Zandonella, C. UC Berkeley Researchers Create World's Smallest Rotary Internal Combustion Engine. University of California Berkeley, 2 April 2001. [Online]. http://www.berkeley.edu/news/media/releases/2001/04/02_engin.html.

17. Epstein, A.H. \& Senturia, S.D., 1997. Macro Power from Micro Machinery. Science, 276, no. 5316, p. 1211.

18. Landau, L. \& Lifshitz, E., 1987. Fluid Mechanics. Oxford: Pergamon Press.

19. Zeldovich, Y. B., Barenblatt, G., Librovich, V. \& Makhviladze, G., 1985. The Mathematical Theory of Combustion and Explosions. New York: Consultants Bureau.

20. Darrieus, G., 1983. La Technique Modern. Paris, Congres de Mechanique Applique.

21. Landau, L., 1944. On The Theory of Slow Combustion. ACTA Physico-Chimica URSS, 19, p. 77.

22. Pelce, P. \& Clavin, P., 1982. Influence of Hydrodynamics and Diffusion upon the Stability Limits of Laminar Premixed Flames. Journal of Fluid Mechanics, 124, pp. 219-237.

23. Bychkov, V.V., Golberg, S.M., Liberman, M.A. \& Eriksson, L.E., 1996. Propagation of Curved Flames in Tubes. Physical Review E, 54, pp. 3713-3724.

24. Bychkov, V.V., 1998. Nonlinear Equation for a Curved Stationary Flame and the Flame Velocity. Physics of Fluids, 10, p. 02091. 
25. Clavin, P. \& Garcia, P., 1983. The Influence of the Temperature Dependence of Diffusivities on the Dynamics of Flame Fronts. Journal de Mécanique Théorique et Appliquée, pp. 245263.

26. Kobayashi, H. \& Kawazoe, H., 2000. Flame Instability Effects on the Smallest Wrinkling Scale and Burning Velocity of High-Pressure Turbulent Premixed Flames. Proceedings of the Combustion Institute, 28, pp. 375-382.

27. Bychkov, V.V., Golberg, S.M., Liberman, M.A., Kleev, A.I. \& Eriksson, L.E., 1997. Numerical Simulation of Curved Flames in Cylindrical Tubes. Combustion Science and Technology, 129, pp. 217-242.

28. Searby, G., 1992. Acoustic Instability in Premixed Flames. Combustion Science and Technology, 81, pp. 221-231.

29. Clanet, C. \& Searby, G., 1996. On the "Tulip Flame" Phenomenon. Combustion and Flame, 105, pp. 225-238.

30. Bychkov, V.V., Akkerman, V., Fru, G., Petchenko, A. \& Eriksson, L.E., 2007. Flame Acceleration at the Early Stages of Burning in Tubes. Combustion and Flame, 150, pp. 263276.

31. Shelkin K.I., 1940. Influence of the Wall Roughness on Initiation and Propagation of Detonation in Gases. Zh. Eksp. Teor. Fiz. 10, pp. 823-827.

32. Brailovsky, I. \& Sivashinsky, G.I., 2000. Hydraulic Resistance as a Mechanism for Deflagration-to-Detonation Transition. Combustion and Flame, 122, pp. 492-499.

33. Kagan, L. \& Sivashinsky, G. I., 2003. The Transition from Deflagration-to-Detonation in Thin Channels. Combustion and Flame, 134, pp. 389-397.

34. Ott, J.D., Oran, E.S. \& Anderson, J.A., 2003. A Mechanism for Flame Acceleration in Narrow Tubes. American Institute of Aeronautics and Astronautics, 41, pp. 1391-1396.

35. Bychkov, V., Petchenko, A., Akkerman, V. \& Eriksson, L.E., 2005. Theory and Modeling of Accelerating Flames in Tubes. Physical Review E, 72, p. 046307. 
36. Akkerman, V., Bychkov, V.V., Petchenko, A. \& Eriksson, L.E., 2006. Accelerating Flames in Cylindrical Tubes with Non-Slip at the Walls. Combustion and Flame, 145, pp. 206-219.

37. Valiev, D.M., Bychkov, V.V., Akkerman, V. \& Eriksson, L.E., 2009. Different Stages of the Flame Acceleration from Slow Burning to Chapman-Jouguet Deflagration. Physical Review E, 80, p. 036317.

38. Akkerman, V., Law, C.K., Bychkov, V.V. \& Eriksson, L.E., 2010. Analysis of Flame Acceleration induced by Wall Friction in Open Tubes. Physics of Fluids, 22, p. 053606.

39. Roy, G.D., Frolov, S.M., Borisov, A.A. \& Netzer, D.W., 2004. Pulse Detonation Propulsion: Challenges, Current Status, and Future Perspective. Progress in Energy and Combustion Science, 30, pp. 545-672.

40. Cicarelli, G., Fowler, C.J. \& Bardon, M., 2005. Effect of Obstacle Size and Spacing on the Initial Stage of Flame Acceleration in a Rough Tube. Shock Waves, 14(3), pp. 161-166.

41. Bychkov, V.V., Valiev, D. \& Eriksson, L.E., 2008. Physical Mechanism of Ultra-Fast Flame Acceleration. Physical Review Letters, 101, p. 164501.

42. Valiev, D.M., Bychkov, V.V., Akkerman, V., Law, C.K. \& Eriksson, L.E., 2010. Flame Acceleration in Channels with Obstacles in the Deflagration-to-Detonation Transition. Combustion and Flame, 157, pp. 1012-1021.

43. Hackert, C.L., Ellzey J.L. \& Ezekoye O.A., 1998. Effects of Thermal Boundary Conditions on Flame Shape and Quenching in Ducts. Combustion and Flame, 112, pp. 73-84.

44. Daou, J. \& Matalon, M., 2002. Influence of Conductive Heat-Losses on the Propagation of Premixed Flames in Channels. Combustion and Flame, 128, pp. 321-339.

45. Norton, D.G. \& Vlachos, D.G., 2003. Combustion Characteristics and Flame Stability at the Microscale: a CFD study of Premixed Methane/Air Mixtures. Chemical Engineering Science, 58, pp. 4870-4882.

46. Kagan, L., Valiev, D., Liberman, M., Gamezo, V., Oran, E., \& Sivashinsky, G., 2006. Effect of Hydraulic Resistance and Heat Losses on the Deflagration-to-Detonation Transition. In Pulse Detonation Engine, Ed. S. Frolov, Torus Press Ltd, Moscow, 51-62. 
47. Gamezo, V.N., \& Oran, E.S., 2006, Flame Acceleration in Narrow Tubes: Effect of Wall Temperature on Propulsion Characteristics. AAIA Paper AIAA-2006-1134, American Institute of Aeronautics and Astronautics, Reston, VA.

48. Williams F.A., 1985. Combustion Theory. Benjamin CA.

49. Lee, J.H.S., 1984. Dynamic Parameters of Gaseous Detonations. Annual Review of Fluid Mechanics, 16, pp. 311-336.

50. Gavrikov, A.I., Efimenko, A.A. \& Dorofeev S.B., 2000. A Model for Detonation Cell Size Prediction from Chemical Kinetics. Combustion and Flame, 120, pp. 19-33.

51. Dorofeev, S.B., Sidorov, V.P., Dvoinishnikov, A.E., \& Breitung, W., 1996. Deflagration to Detonation Transition in large confined volume of lean hydrogen-air mixtures. Combustion and Flame, 104, pp 95-110

52. Shepherd, J. E., 1986. Chemical Kinetics of Hydrogen-Air-Diluent Detonations. Progress in Astronautics and Aeronautics, 106, pp.263-293.

53. Oppenheim, A.K. \& Soloukhin, R.I., 1973. Experiments in Gasdynamics of Explosions. Annual Review of Fluid Mechanics, 5, pp. 31-58.

54. Peraldi, O., Knystautas, R. \& Lee, J.H., 1986. Criteria for Transition to Detonation in Tubes. Twenty-first Symposium (International) on Combustion, pp. 1629- 1637.

55. Kuznetsov, M., Alekseev, V., Matsukov, I. \& Doroseev, S., 2005. DDT in a Smooth Tube Filled with a Hydrogen-Oxygen Mixture. Shock Waves, 14, pp. 205-215.

56. Dorofeev, S.B., Sidorov, V.P., Kuznetsov, M.S., Matsukov, I.D., \& Alekseev, V.I., 2000. Effect of Scale on the Onset of Detonations. Shock Waves, 10, pp. 137-149.

57. Zeldovich, Ya.B., Librovich, V.B., Makhviladze, G.M. \& Sivashinsky, G.I., 1970. On the development of detonation in a non-uniformly preheated gas. Astronautica Acta, 15, pp.313321.

58. Khokhlov, A.M., Oran, E.S. \& Wheeler, J.C., 1997. A theory of deflagration-to-detonation transition in unconfined flames. Combustion and Flame, 108, pp.503-517.

59. Akkerman, V., Demirgok, B., Bychkov, V., Valiev, D., Law, C.K. \& Wu, M., 2015. Analysis of Ethylene-Oxygen Combustion in Micro-Pipes. $9^{\text {th }}$ U.S. National Combustion Meeting, May 17 -20, Cincinnati, OH, USA. 
60. Wu, M \& Wang, C., 2011. Reaction Propagation Modes in Millimeter-Scale Tubes for Ethylene/Oxygen Mixtures. Proc. of the Combustion Institute, 33, pp 2287-2293.

61. Johansen, C. \& Cicarelli, G., 2009. Visualization of the Unburned Gas Flow Field ahead of an Accelerating Flame in an Obstructed Square Channel. Combustion and Flame 156, pp 405-416.

62. Ferguson, C. R., \& Keck, J. C., 1977. On Laminar Flame Quenching and its Application to Spark Ignition. Combustion and Flame 28, pp 197 - 205.

63. Demirgok, B., Ugarte, O., Valiev, D. \& Akkerman, V., 2015. Effect of Thermal Expansion on Flame Propagation in Channels with Nonslip Walls. Proceedings of the Combustion Institute 35 (1), pp 929 - 936.

64. Dion, C.M., Demirgok, B., Akkerman, V., Ugarte, O., Valiev, D. \& Bychkov, V., 2015. Flame Propagation in Channels with Cold Walls: Heat Loss versus Acceleration. Submitted to Combustion and Flame.

65. Bychkov, V., 2015. Private Communication. 